
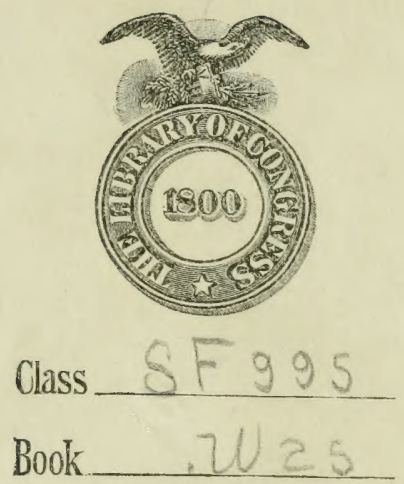

Copyrightit No

COPYRIGHT DEPOSIT. 


DISEASES OF DOMESTICATED BIRDS 


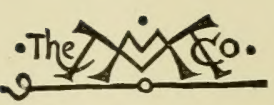

THE MACMILLAN COMPANY

NEW YORK - BOSTON - CHICAGO - DALLAS ATLANTA - SAN FRANCISCO

MACMILLAN \& CO., Limited

LONDON - BOMBAY - CALCUTIA

MELBOURNB

THE MACMILLAN CO. OF CANADA, LTD. TORONTO 


\section{DISEASES \\ OF DOMESTICATED BIRDS}

BY

ARCHIBALD ROBINSON WARD, B.S.A., D.V.M.

Director, Research Department, The Jensen-Sulsbery

Laboratories, Kansas City, Missouri

AND

BERNARD A. GALLAGHER, D.V.M.

Bacteriologist, Bureau of Animal Industry, United States

Department of Agriculture, Washington, D. C.

WITH INTRODUCTION BY

VERANUS ALVA MOORE, B.S., M.D., V.M.D.

Professor of Comparative Pathology, Bacteriolugy and Meat Inspection,

New York State Veterinary College at Cornell University,

and Director of the College

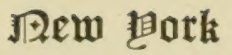 \\ THE MACMILLAN COMPANY \\ 1920}

Ali rights reserved 


$$
\begin{array}{r}
5 F 995 \\
\text { W25 }
\end{array}
$$

Copyright, 1920,

BY THE MACMILLAN COMPANY

Set up and electrotyped. Published, January, 1920

FEB -31920

(C) CI.A559635 


\section{PREFACE}

The writing of this book has been prompted by a conviction held by the authors, regarding the magnitude of the losses experienced by poultry producers as a result of preventable infectious diseases and parasitic infestations.

It is believed that the importance of these losses warrants the effort expended in presenting the essential facts concerning poultry diseases to meet the needs of veterinary practitioners, veterinary students and poultrymen.

The title of the book indicates a slight broadening of scope beyond the field of a work on diseases of poultry. Material included deals with the ostrich, cage birds and wild birds in semi-domestication in zoological gardens. Chapters dealing with anatomy of birds, and killing of poultry have been included.

The peculiarities of diseases of birds are indicated by the emphasis laid on certain features of the subject matter. In general, skill in diagnosing diseases of birds by observing symptoms is of less importance than ability to recognize the identity of disease at autopsy. Symptoms are slightly characteristic, but autopsy material is freely available. Consequently more facts concerning details of gross lesions are given than those relating to symptoms. In many cases, particularly in the septicemias, symptoms and lesions are not distinctive, and fine distinctions of etiology must be made by a bacteriologist. On this account considerable material useful to a bacteriologist alone has been included.

Available sources of information are indicated in the references appended to each chapter and by specific references to authors in the text. Special acknowledgment is made for assistance obtained from Neumann's "Parasites et Maladies Parasitaires des Oiseaux Domestiques" in the preparation of the chapters on parasites. Much material concerning the less common parasites has been drawn from this book and Neumann's zoological nomenclature has been largely followed.
A. R. W.
B. A. G. 



\section{INTRODUCTION}

The statement is accepted that diseases are the greatest hindrance to the development of animal and poultry husbandry. Measures for the prevention, successful treatment or control of these maladies must come from those who understand their nature. It is gratifying, therefore, that veterinarians, and others interested in the subject, are to have access to a volume dealing with the diseases of poultry, prepared by men of long experience in the study of the phenomena of disease. Likewise, poultry raisers will appreciate a treatise setting forth the known facts concerning the maladies which sooner or later may affect their flocks. A book of this kind will be of indispensable value in eliminating many of the misconceptions regarding the diseases of poultry and in pointing out the things that are known and the precautions that must be observed in maintaining healthy flocks.

The extent of the poultry industry in the United States is little appreciated by veterinarians. There is no other species in the animal kingdom that contributes to the material support of so large a number of people as the domesticated fowl. In addition turkeys, geese, ducks and pigeons are significant factors in food production while the ostrich and other birds play an important part in ornamentation. In the Hartz Mountains, the raising of canary birds for the amusement and entertainment of people is an important industry.

The barnyard fowl plays a large part in the food supply of the country. The extent of the poultry business can be approximated from the fact that in 1910 there were $5,578,525$ farms in the United States that reported a total of $280,350,000$ fowls with a value of $\$ 140,205,000$. With the prices at the present time, their value would be treble that of 1910 . In 1915, the United States exported $20,784,000$ dozen eggs but it importer over 3,000,000 dozen and over $8,000,000$ pounds of egg yolks and frozen eggs. These figures indicate the large number of people who are financially interested in the poultry business.

The raising of turkeys and other species is also significant. In 1910 there were in the United States 3,68s,000 turkers with a value 
of $\$ 6,605,000 ; 2,906,000$ ducks valued at $\$ 1,567,000 ; 4,431,000$ geese valued at $\$ 3,194,000 ; 1,765,000$ guinea fowls valued at $\$ 613,000 ; 2,730,000$ pigeons with a value of $\$ 762,000 ; 6,458$ pea fowls estimated at $\$ 18,000$; and 5,361 ostriches valued at $\$ 1$,696,000 . In 1915, this country imported ostrich and other feathers to the value of $\$ 2,500,000$. Besides, there is a large business in song and other birds maintained as pets.

In certain localities, the raising of one or more species of poultry constitutes the essential occupation of many of the people. The productivity of domestic fowls is greater than that of any other species of animals in proportion to their value. The price of the annual products from a flock of ducks, hens or turkeys may be many times larger than the cost of the original flock.

There is no other form of animal life that suffers more from the ravages of disease than fowls. The losses from the general maladies are heavy and those from the infectious ones are sometimes appalling. Various estimates have been made of the annual losses occasioned by these causes. They vary from 10 to 30 per cent. Hens in particular suffer from a wide range of diseases and parasitisms. Some of these are veritable plagues and at times are the cause of serious losses. In 1903, while studying poultry diseases in a locality where eggs and chickens were the chief articles of commerce, it was not unusual to learn of individual losses ranging from a few hundred to several thousand dollars due to roup or diphtheria. Other large flocks were decimated by tuberculosis. While it is easy to understand that heavy losses may occur in localities where fowls are kept in large numbers, it should be recognized that they are proportionately heavy in smaller flocks. In such circumstances, the death of a few hens is often the cause of much privation if not actual suffering among their owners who are dependent upon the products of their fowls for the necessities of life.

The general diseases of poultry present many points of interest, although they have received relatively little attention. The general pathology of the feathered tribes is not well understood. Little seems to be known of their tissue reactions to injury. Their resistance to ordinary wound infections differs strikingly from that of mammals. Pasteur suggested that this might be due to their higher body temperature. On the other hand, the infectious diseases and parasitisms have been more carefully studied. The findings of many valuable researches are recorded on the nature of the more serious ones. Certain of them, such as bacillary white diarrhea and 
the parasitisms, have been elucidated sufficiently to enable reterinarians or poultry owners to prevent them. Others that cause heavy losses, such as roup or diphtheria and tubereulosis, can be controlled. When this is done, millions of dollars worth of poultry that is now lost will be saved. This can be done as soon as the technical knowledge, now available, is utilized to its full extent.

It is believed that veterinarians can render a valuable service to this heretofore professionally neglected, yet important industry, especially in localities conspicuous for the raising of poultry. Because of the relatively small value of the individual, and the large numbers to be dealt with, methods for preventing disease must be applied to the flock. With the exception of the more valuable birds, individual treatment may not be practicable, but the principles of sanitation and the preventive measures are as helpful here as elsewhere in the raising of animals. Veterinarians should realize that the treatment, prevention or control of diseases in poultry, as well as in quadrupeds, is primarily an economic question and that a score of dollars saved by preventing or treating diseases in fowls is just as essential as the saving of a like amount in sheep or calves. This book, dealing with the diseases and the parasites of poultry, will be of much aid to veterinarians who wish to render full service to their communities.

V. A. MLore. 



\section{TABLE OF CONTENTS}

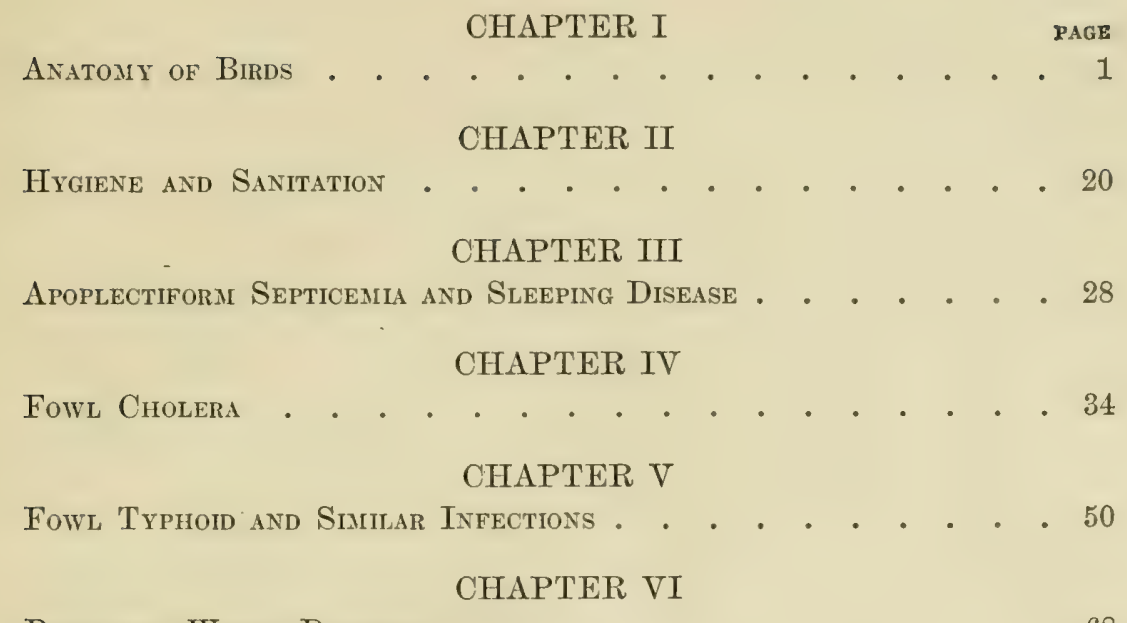

Bacillary White Dlarrhea

CHAPTER VII

Fowl Plague . . . . . . . . . . . . . . 81

CHAPTER VIII

Avian Tuberculosis . . . . . . . . . . . . . . . 88

CHAPTER IX

Avian Diphtileria and Bird Pox . . . . . . . . . . 96

CHAPTER $\mathrm{X}$

Aspergillosis and Favus . . . . . . . . . . . . . 111

CHAPTER XI

Infectious Entero-Hepatitis of Turkeys . . . . . . . . . 120

CHAPTER XII

Coccidiosis . . . . . . . . . . . . . . . . . . 127

CHAPTER XIII

Spirochetosis and Rare Infectious Diseases . . . . . . . . 135 
xii

CONTENTS

CHAPTER XIV

Leukemia and Pseudo-Leutemia.

CHAPTER XV

General Diseases . . . . . . . . . • . . . . . 150

\section{CHAPTER XVI}

Internal Parasites 178

CHAPTER XVII

External Parasites . . . . . . . . . . . . . 208

CHAPTER XVIII

Infectious Diseases and Parasites of the Ostrich . . . . . . 226

CHAP'TER XIX

Infectious Diseases of Geese and Duchs . . . . . . . . . 242

CHAPTER XX

Infectious Diseases of Canary Birds . . . . . . . . . . 252

CHAPTER XXI

Tumors in Fowls . . . . . . . . . . . . . 261

CHAPTER XXII

TOXICOLOGY . . . . . . . . . . . . . . . . . . 276

CHAPTER XXIII

Surgichl Diseases . . . . . . . . . . . . . . . 295

CHAPTER XXIV

CAPONIZING •. . . . . . . . . . • . . . 302

CHAPTER XXV

Kílling Poultry . • . • . . . . • . • . . . 314 


\title{
DISEASES OF DOMESTICATED BIRDS
}

\author{
CHAPTER I \\ ANATOMY OF BIRDS
}

The structure of birds differs in many respects from that of mammals and deserves a special description. The aim of the authors is to bring to the attention of the reterinarian the more important differences which are observed in the structure of domesticated birds as compared with that of domesticated mammals. The subject will be considered under the following sub-divisions. 1. Skeleton, 2. Musculature, 3. Circulatory system, 4. Nervous system, 5. Alimentary system, 6. Respiratory system, 7. Urinary system, 8. Generative system, and 9. The skin and its appendages.

\section{SKELETON}

Skull. The bones of the skull become united shortly after hatching and lose their individual identity except in the case of the ascending processes of the premaxillary and nasal bones which are not rigidly joined to the frontal bone but through their cartilaginous attachment permit the elevation of the superior mandible. The inferior processes of the premaxillary bone, the supermaxillaries and the palatine bones which form the roof of the palate do not meet in the median line, with the result that the palate presents an elongated opening or cleft.

Vertebral column. The cervical vertebræ vary in number in the different species. Statements of anatomists differ concerning the number, because of difficulties experienced in defining cervical and thoracic vertebre. The atlas or first cervical vertebra is ring-like in form and articulates with the occipital bone of the skull by means of a deep concarity into which the condyle of the occipital bone is received. This articular arrangement and that of the other cervical vertebre gives the head and neck great mobility. The articular surface is concave from side to side and convex in a dorso-ventral direction on the anterior extremity of each vertebra, presenting a saddle shaped appearance. The posterior extremity is the reverse, that is, concave dorso-ventrally and convex from side to side. 


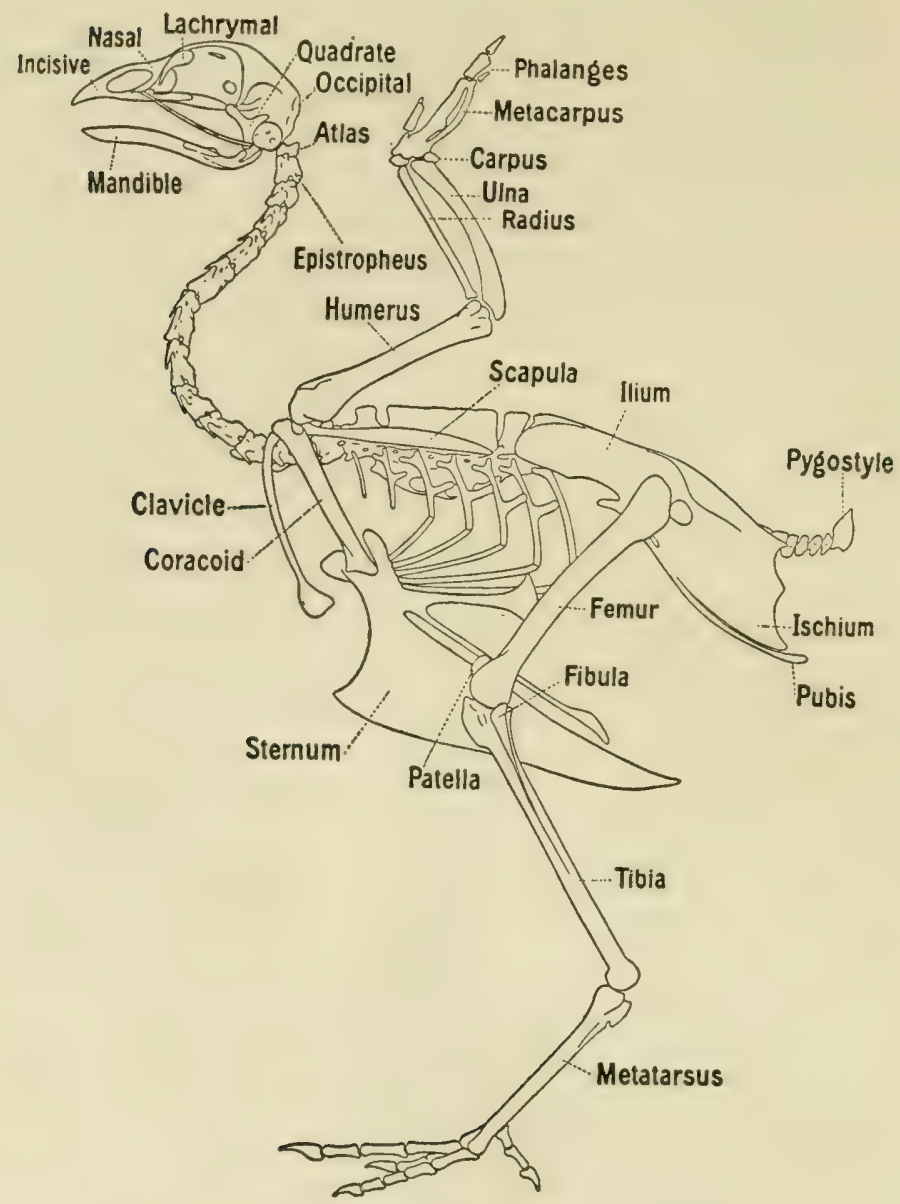

FIG. 1. The skeleton of the fowl. (Bradley)

The thoracic vertebræ are nearly always fused together by the consolidation of their spinous and transverse processes. The first may be free and the last may be fused with the lumbar vertebræ.

The lumbar and sacral regions of the vertebral column show no special line of demarcation. The fourteen vertebræ are consolidated into one bony mass which carries ridges on its ventral surface representing the transverse processes of the original independent vertebræ.

The coccygeal vertebræ are seven in number. The last is the largest, is flattened on both sides and curres in a dorsal direction 
at the end. It is known as the pygostyle. The coccygeal vertebre are movable upon each other.

Ribs. These bones articulate dorsally with the thoracic vertobre, and ventrally with the sternum by means of the inferior ribs, except in the case of the first two which do not reach the sternum. The last rib is often united to the one adjoining and does not connect with the sternum. Some of the ribs have flat uncinate processes which extend from the posterior border and lie over the external face of the following rib near its middle.

The sternum is highly developed in birds and forms the floor of the thoracic cavity and to some extent, of the abdominal cavity. It is concave on its dorsal or internal face and convex on its ventral or external face. In fowls and pigeons a deep ridge extends along the median line of the ventral face. This is known as the brisket or keel. It serves to increase the area of attachment for the powerful breast muscles. The sternum of the duck and goose does not present as large a keel as that of the fowl and pigeon, but owing to the greater breadth of the sternum and more solid construction the area for muscular attachment is as great. In the fowl two long slender lateral processes are formed on each side as a result of deep notches which extend anteriorly into the body of the sternum. The lateral external process is shorter than the lateral internal process and covers the last two ribs with a broadened terminal bony plate. The lateral borders of the body of the sternum support articular facets for the reception of the inferior ribs. The anterior border presents an eminence, the episternal process to which the furculum (wishbone) is attached by a ligament. The articular surfaces for the coracoids are located on each side of the episternal process on the anterior border.

The internal surface of the sternum exhibits several openings through which air is passed to the interior of the bony structure.

Bones of the wing. These comprise the scapula, coracoid, and clavicle in the shoulder region; the humerus in the arm; the radius and ulna in the forearm; two carpal bones, the radio-carpal bone and ulno-carpal bone; one carpo-metacarpal bone, and three digits the second of which has two phalanges.

The scapula is long, narrow, thin and curved like a sabre. It extends caudad over the ribs to near the pelvis. Anteriorly it is mited with the coracoid with which it forms an articular earity for the reception of the head of the humerus. The coracoid also articulates with the sternum by its ventral extremity. It is rod 
shaped and extends in a posterior direction obliquely ventrad from scapular to sternum. The coracoid at its ventral extremity presents an opening for the passage of air to the internal air space. The clavicle is attached to the coracoid at its superior end. It extends ventrad and caudad and curves in to the median line at the point of the sternum where the two clavicles unite in a flattened plate and become attached to the episternal process by a ligament. The united clavicles form a V or $\mathrm{U}$ shaped bone termed the fork or fureulum. This bone represents an elastic spring and serves to prevent the wings from approaching each other during contraction of the depressor muscles.

The humerus is long in palmipeds and the gallinæ, and short in the pigeon. It is stout, slightly curved and presents a pneumatic foramen near its articular head.

The ulna is much larger than the radius and is separated from it except at the extremities, where the two are mited by ligamentous bands. The olecranon process of the ulna is only slightly developed.

The two carpal bones represent fusions in embryonic life, of the four bones of the proximal row. The distal carpal row of three bones fuse with the metacarpal bones.

Bones of the leg. These are the pelvic or hip bone; the femur or thigh bone; the patella, tibio-tarsus and fibula in the lower leg region; a tarso-metatarsal bone, and four digits.

The pelvic bone is made up of three bones as in mammals, the ilium, ischium and pubis. It differs from the mammalian bone in that the pelvic girdle is not complete on the ventral floor, the two pubic bones failing to meet in the center to form the pubic symphysis. This arrangement facilitates the passing of the egg through the cavity. The ilium is the largest segment and extends along the vertebral column in the lumbar and sacral regions. It is consolidated with the last two thoracic vertebre and the lumbar and sacral vertebre, this extensive fusion giving the pelvis a rigidity which compensates for the lack of a pubic union. The ischium partly forms the side of the pelvic cavity. The pubis is mainly attached to the ischinm along the lower border and bends inward to form the incomplete floor of the pelvic cavity. The ilium, ischium and pubis are united at the acetabulum or articular cavity for the reception of the head of the femur.

The femur articulates at its distal extremity with the patella, tibia and fibula. The latter bone is united to the tibia, but does not extend to its distal articulation. The tibia is comparatively long. 
It has two articular surfaces on its distal extremity which articulate with the tarso-metatarsal bone. The tarsus is absent in birds. In males the tarso-metatarsus exhibits a slightly curved conical projection near the distal third for the support of the spur.

Four digits articulate with the distal extremity of the tarso-metatarsus. Three are directed radially cephalad. The internal digit has three phalanges; the middle, four and the external, five. The fourth digit is directed caudad and has two phalanges succeeding a rudimentary first metatarsal bone attached to the metatarsus by fibro-cartilaginous tissue. The distal bone of each digit is covered by a horny claw.

\section{MUSCULAR SYSTEM}

In general the muscles of birds correspond to those of mammals, with such modifications as are most suited to the mechanism of avian structure. The tendency, especially in turkeys and fowls, is toward extensive ossification of the muscle tendons. This is particularly noticeable in the tendons of the legs but may also be observed in the wing and neck in some cases. The fowl and turkey also present a marked contrast in the appearance of the muscles of the breast and wings as compared with those of the legs. The breast and wing muscles are white, due to the relatively small amount of sarcoplasm in the muscle fibers. The red muscles are rich 'in sarcoplasm. White muscles are more powerful but have less endurance than red ones. Free flying birds have red pectoral or breast muscles.

The pectoral muscles are two in number, the superficial or great and the deep or small pectoral. The former depresses the wing and the latter elevates it. These muscles are very massive. The great pectoral muscle is attached to the clavicle, to the sternal crest near its border, to the posterior lateral process of the sternum and to the last ribs. It is inserted into the lateral tuberosity near the head of the humerus. It is this muscle that propels the bird in flight.

The small pectoral muscle originates from the portion of the ventral face of the sternum not occupied by the great pectoral and also from the clavicle and membrane between the latter and the sternum. Its tendon is inserted into the humerus opposite that of the great peetoral muscle after passing through the foramen triosseum which acts as a pulley. This foramen is formed by the uniom of the scapula, coracoid and furculum.

The diaphragm does not exhibit the muscular derelopment of that 
of mammals. It is principally membranous with fine muscular bundles attached to the ribs. Two thin, delicate membranes compose the diaphragm and divide it into two planes, a pulmonary and a thoracic-abdominal plane. The former is spread over the inferior face of the lungs and is attached to the ribs on its border. It assists in respiration. The thoracic-abdominal plane has its origin on the dorsal spine in common with the pulmonary plane. It is convex anteriorly and extends to the sternum, dividing the body cavity into a thoracic and an abdominal cavity. It also assists in respiration and is particularly concerned with the abdominal air sacs.

The pectineus muscle assists in the mechanical flexion of the toes in the act of perching on the roost. It originates on a tuberosity of the hip bone in front of the acetabulum and is continued by a long thin tendon to a short distance distad from the head of the tibia where it joins the flexor perforatus of the three anterior digits.

\section{CIRCUIAATORY SYSTEM}

The heart of birds is cone shaped, short and wide in the ostrich, elongated and possessing a more acute apex in the common fowl. It is placed in the anterior extremity of the thoracic cavity. Three veins, two anterior venæ cavæ and one posterior vena cava, terminate in the sinus of the right auricle. A peculiarity of the heart of birds is the absence of a tricuspid valve at the right auriculo-ventricular opening. Instead, a strong muscular fold which becomes closely applied to the ventricular septum during systole prevents regurgitation of blood into the auricular cavity. Two pulmonary reins empty into the left auricle. The left auriculo-ventrieular opening is provided with a bi-cuspid or mitral valve. The pulmonary artery and aorta each has a semilunar valve.

Arteries. The aorta gives off two innominate arteries, right and left brachio-cephalic, on leaving the heart and then passes orer the right bronchus to become the descending or dorsal aorta. It continues along the spine, giving off numerous branches, to the coccrgeal region where it separates into three terminal arteries.

The two carotids leave the innominate arteries and curve toward the ventral median line of the neck where they ascend in close proximity to each other to near the head. At the angle of the jaw each carotid divides into the external and internal carotid arteries. The arteries, especially those of the head and viscera, are distinguished 
by their frequent anastomoses. This is also true of the veins of birds.

Veins. The blood is returned to the heart by three trunks, the two anterior venæ cavæ and the posterior vena cava. The right jugular exeeeds the left in size. The two communicate with each other by means of a cross branch at the base of the cranium.

Lymphatics. The lymphatics of birds do not form two strata, a superficial and a deep seated, as in mammals. The latter only is observed. Lymphatic glands are few. The most conspicuous are located at the base of the neck and in the anterior thoracic cavity. They are replaced by plexuses of lymphatic vessels surrounding the principal blood ressels. From the aortic plexus arise two thoracic ducts which pass cephalad on the ventral surface of the lungs and terminate respectively in the jugular vein of the same side.

\section{NORMAL AVIAN BLOOD}

In birds as in mammals the blood is composed of a straw colored fluid or plasma in which float several types of blood corpuscles. One important difference between avian and mammalian blood, excepting the camel, is the presence of nuclei in the red corpuscles of the former. The formed elements or corpuscles are divided into two main divisions, the red cells or erythrocytes and the white cells or lencocrtes. These elements vary greatly in number in different birds, and even in the same bird at different ages. Each cubic millimeter of an adult fowl's blood contains approximately 3,250,000 red cells and 26,000 white cells, giving a percentage relation of 125 to 1 . In certain pathologic conditions this relation is markedly changed, the red cells rapidly decreasing and the white cells increasing in numbers.

Red blood corpuscles or erythrocytes are straw colored, nucleated, oral, flattened dises, about 12 microns in length and $7.3 \mathrm{mi-}$ crons in widtli. The nuclei take a deep blue stain with methylene blue, or Giemsa. The surrounding protoplasm takes a light rellow with methylene blue and a pink color with Giemsa's stain.

White blood corpuscles or leucocytes are nucleated, colorless cells, rounded or irregular in form. They exhibit ameboid movement in the blood stream, some being more actively motile than others. The variations in their size, character of the nuclei, presence of granules and character of the inclosed granules differentiate these cells into five varieties. These varieties with their percentage ratio are as follows: 


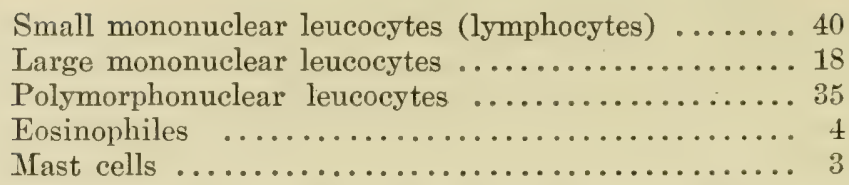

Lymphocytes. In these blood cells the nucleus is rounded or oval and almost completely fills the cell. It stains a deep blue with Giemsa. The narrow band of cell body stains a lighter blue and is not readily differentiated from the nucleus by ordinary staining. The lymphocytes are from 5 to 7 microns in diameter.

Large mononuclears have a round or oval nucleus surrounded by a well defined border of cell protoplasm. The nucleus takes a deep blue stain, the cell body a pale blue. The cell diameter is 10 to 15 microns.

Polymorphonuclears have the appearance of having several nuclei held together by threads or nuclear bands. They contain considerable protoplasm inclosing rod shaped or rounded granules. The nucleus stains blue, the protoplasm, a lighter color and the granules violet or brownish red with Giemsa's stain. Diameter, 10 to 15 microns.

Eosinophiles have an irregular nucleus. The cell protoplasm is filled with rounded granules which stain a bright red with Giemsa's stain. The nucleus takes a blue stain and the cell body a pale blue. Diameter, 12 microns.

Mast cells have a lobulated nucleus which stains a deep blue. The cell protoplasm takes a pale stain. The granules are small and rounded and stain a dull violet with Giemsa and a deep blue with methylene blue. Diameter, 8 to 10 microns.

Other blood cells of less importance which may be observed are as follows:

Blood platelets are small colorless dises with granular centers. They are 3 to 4 microns in diameter and are usually found in small heaps of 6 to 8 cells.

Shadow cells contain a rounded or broad oval nucleus which takes a deep blue stain. The cell body is colorless and not well defined or irregular in outline. These apparently represent red blood corpuscles undergoing degeneration. The stroma of the corpuscle loses its pigment, becomes colorless and gradually disappears leaving the nucleus partially or wholly separated. They are especially numerous in pathologic blood.

Microblasts and macroblasts are found in the blood of young 
fowls. These represent red corpuscles which are smaller and larger respectively than the average red corpuscle.

Myelogenous leucocytes appear frequently in roung fowls. They are like mast cells except that their protoplasm is vacuolated.

NERVOUS SYSTEM

The nervous system of birds, with few exceptions, corresponds with that of mammals. However, there are several modifications which are of considerable interest. The cerebral hemispheres of the brain are well developed but have a smooth surface in contrast to the convoluted surface observed in the cerebrum of mammals. The cerebellum is conspicuous by the smallness of its lateral lobes. The optic lobes are placed in a lateral inferior position at the sides of the cerebellum. The large size of the optic chiasma and nerres indicates the paramount importance of the visual apparatus. The diameter of an optic nerve is equal to that of the spinal cord in its dorsal region. The olfactory lobes are relatively small. The hippocampi are absent in the cerebrum of birds. An important feature of the nervous system of birds is the absence of nervous tracts leading to the spinal cord from the cerebrum. The cerebrum does however exert an influence over the rest of the nervous system through its nervous tracts connecting it with the other divisions of the brain. The anatomical evidence of the absence of nerrous tracts between the cerebral hemispheres and the spinal cord is suplorted by the absence of any marked defects of movement succeeding the remoral of the cerebrim. The fowl so treated, on recovery from the operation, is not sensitive to light or sound. It fails to pick up grains and must be fed. Movement is not interfered with as the bird constantly walks about aimlessly. Its avoidance of obstacles shows that it is guided by some obscme means. Ability to perch and to maintain its balance is not lost. The weight of the brain of a medium sized fowl is ten grams.

The spinal cord of birds extends into the coccygeal vertebræ. It is cylindrical throughout its length except in the sacral and cerricodorsal regions where two expansions are noted. The dorsal columms of the cord diverge from each other in the sacrum to form the carity known as the sinus rhomboidalis which represents a ventricular dilatation of the spinal canal. The brachial plexus is formed he the last cerveal and first two dorsal spinal nerves in ducks and geese and 
by the last three cervical and first thoracic spinal nerves in fowls, turkeys and pigeons. It furnishes branches to the pectoral region and wing. The pelvic region and legs are supplied by the lumbosacral plexus which is formed by two lumbar and four sacral spinal nerves. The spinal nerves vary in number according to the number of vertebræ present in each species. They arise from the spinal cord by two roots. The anterior or ventral root is motory, the posterior or dorsal, sensory in function. The ganglia on the latter are proportionately large.

\section{ALIMENTARY SYSTEMI}

The alimentary system of birds differs to a considerable degree from that of other animals. There is no provision for mastication of food in the mouth owing to the absence of teeth. The food is taken up by the beak and is immediately passed by the tongue into the pharynx. From there it enters the esophagus and is carried to the crop where it is stored, partially softened, and slowly passed on to the proventriculus or glandular stomach. The gall bladder is absent in the pigeon and ostrich and occasionally in the guinea fowl.

Mouth. The mouth is triangular in shape in such birds as the chicken, turkey, pigeon and canary. In the duck, goose and ostrich the mouth is comparatively long, with parallel borders and rounded anterior extremity. The roof of the mouth or hard palate is divided in the median line from near its anterior extremity to the pharrnx, thus allowing free communication with the nasal passages. The soft palate observed in mammals is absent. The pharynx is relatively voluminous and permits the passage of large grains, or food masses. On its floor is the entrance to the larynx. The esophagus lacks the well developed muscular wall of mammals but relatively has a much greater diameter.

Crop. Gallinaceous birds possess a crop or ingluvies which represents a dilatation of the esophagus near its entrance to the thoracic cavity. Its wall, like that of the esophagus, consists of an onter membrane, a longitudinal muscular layer, a circular muscular layer, and an inner mucous membrane. Passage of its contents to the first stomach is aided by a wide circular muscle in the subcutaneous tissue surrounding the crop. Palmipeds, such as ducks and geese, are not provided with a distinct crop. Instead, the cervical portion of the esuphagus may beerme greatly distended in such a manner as to form a long fusiform reservoir. 
FIG. 2. Diagrammatic representation of the principal internal organs of the fowl. 1, tongue; 2, pharynx; 3, upper portion of esophagus; 4 , crop; 5 , lower portion of esophagus; 6, proventriculus; 7 , gizzard; 8 , origin of duodenum; 9 , first branch of duodenum; 10, second branch of duodenum; 11, origin of floating portion of small intestine; 12, small intestine; $12^{\prime}$, terminal portion of small intestine; 13, free ends of ceca; 14, origin of ceca; 15 , rectum; 16 , cloaca; 17 , vent; 18 , mesentery; 19 , left lobe of liver; 20 , right lobe of liver; 21, gall bladder; 22, spleen; 23, pancreas; 24, lung; (Heart not shown but located approximately here) ; 25 , ovary; 26 , oviduct; 27, kidney; 28, ureter; 29, upper larynx; 30, trachea; 31, lower larynx or syrinx; 32 , bronchus; 33 , cleft palate; 34 , common ori- $A$ fice of eustachian tubes. (Redrawn from Chauveau, with slight additions)
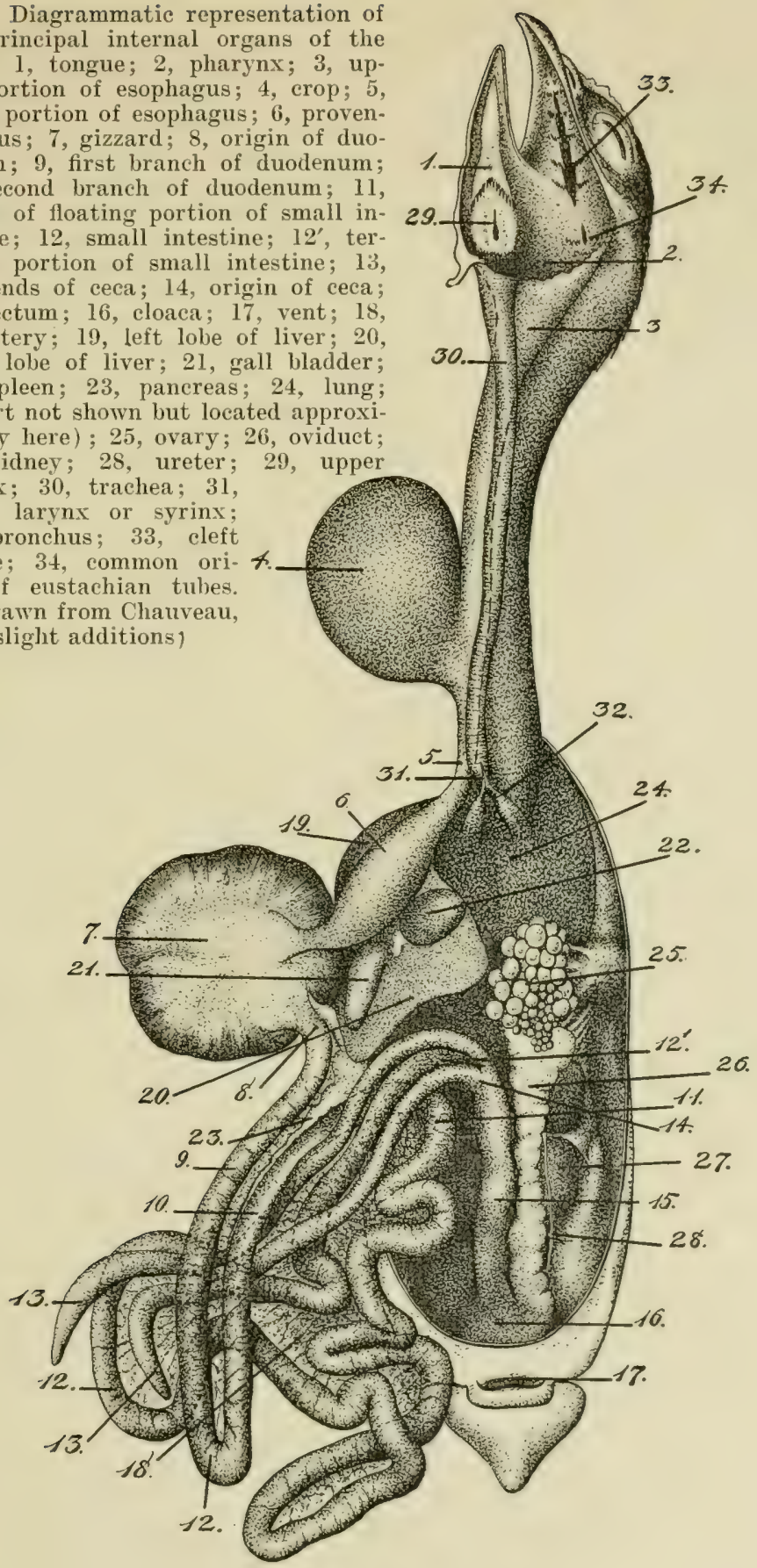
In pigeons the crop is divided into two lateral pouches. In both male and female, marked changes take place in the mucous mem brane shortly before the young are hatched. The membrane becomes thickened, epithelial cells are rapidly proliferated, undergo fatty degeneration and lead to the formation of a milky fluid which aceumulates in the depressions between the ridges of the mucosa. This fluid is used to feed the young during the first days of life.

A certain amount of fluid is secreted by the alveolar glands of the esophagus which together with the water consumed serves to soften grains to some extent and also to produce a slight fermentation of the crop contents. The crop has no secretory glands other than mucous glands. It is connected with the proventriculus or first stomach by a continuation of the esophagus which passes into the thoracic cavity and along the ventral face of the left lung.

Proventriculus. The proventriculus is small and fusiform in shape. Its walls are thick and contain numerous secretory glands which pour out gastric juice. The food is not held in the proventriculus to be digested but after becoming saturated with the gastric secretion is forced into the gizzard where it is triturated by the powerful muscles of this organ with the aid of pebbles picked up by the bird.

Gizzard. The gizzard or ventriculus bulbosus is a dense, muscular organ, flattened on two surfaces, with rounded border. Internally it is lined by a thick, tough, corrugated membrane which incloses a cavity of considerable volume, in which may be found food in various stages of disintegration and numerous pebbles, or bits of stone. Secretion of gastric juice also takes place in the gizzard according to Jobert. However, this organ acts principally in a mechanical way to grind the food and serves as a substitute for teeth. It is more highly developed in grain eating birds than in those subsisting principally on fish or animal food. In strictly carnivorous birds such as eagles, or hawks, the dense muscular wall is absent, or is limited to a portion of the organ, the remainder consisting of a membranous cul-de-sac. When reduced to a semifluid mass the food is released into the duodenum or first portion of the intestine, which joins the gizzard at a point near the entrance of the proventriculus.

Intestines. On learing the gizzard the duodenum extends posteriorly, bends on itself and returns to near its origin. Between its two parallel branches rests the pancreas which gives off its secretion into the lumen of the duodenum near its termination. 
The pancreatic juice acts on the starchy material while the secretion of the proventriculus acts on the proteins in the food. Sueceeding the duodenum are, according to some writers, the other two divisions of the small intestine, the jejunum and ileum. These form several convolutions and are continued by the rectum to the cloaca.

At the point of juncture of the small intestine and rectum, in certain species of birds, are found two elongated diverticula leading from the intestine. These are the ceca or blind pouches. In grown fowls and turkeys they are from $t$ to $\tau$ inches in length and may be distended to a diameter of $1 / 2$ to $3 / 4$ of an inch. Especial importance is attached to them because of the fact that they are the seat of marked lesions in coccidiosis of chickens, and of turkers, and in infectious entero-hepatitis of turkeys. In some species of wild birds, the ceea may reach a length of 10 inches while in others they are absent or merely rudimentary. Their function is apparently the retention of food material which has not been absorbed in passage through the small intestine.

The cloaca serves as a common cavity for the entrance of the intestine, ureters, oviduct in the female, and vasa deferentiæ in the male. It is divided into three parts. The rectum enters the first part, the ureters and genital canals open on the lateral faces of the second and the bursa of Fabricus, a blind sac or glandular carity, opens on the dorsal wall of the third or outer division. The bursa of Fabricus may disappear in adults. The one oviduct in the female opens on the left side of the second division. The material from the intestine and ureters is held in the cloaca to form the characteristic droppings and is then passed out through the anus or vent.

Mucosa of the alimentary canal. The alimentary canal is lined internally by a mucous membrane which is thick, and composed of many layers of cells in the mouth, pharrnx, esophagus and crop. The mucosa is thin, and composed of one layer of columnar epithelial cells in the proventriculus, gizzard and intestine. In the gizzard the membrane is covered by a horny surface layer. In the submucous tissue of the mouth and pharynx are found several pairs of glands. These, however, are not considered as true salivary glands but produce a muenus secretion. The esophagus exhibits numerous alveolar secretory glands in the submucous tissue. The glandular stomach is conspicuous for the layer of tubular secretory glands which are set perpendicularly to the mucous membrane and which pour their secretion of galstric juice into the interior of the stomach. Over the mucous membrane of the 
gizzard is a tough horn-like covering formed by the hardening of the products secreted by the underlying simple glands.

The mucous membrane of the intestine presents innumerable cylindrical elevations known as villi. It is through these that the digested food is assimilated and carried by means of the chylevessels or lacteals to the portal vein and thence to the liver. $\mathrm{Nu}$ merous goblet cells for the secretion of mucin are present in the villi.

Liver. This organ is dark brown in color and composed of a right and left lobe, the former being the larger. The right lobe has a gall bladder on its ventral surface, while the bile from the left lobe is poured directly into the duodenum through a separate bile duct, the hepatic or choledic duct, which empties a short distance behind the two pancreatic ducts. The cystic duct opens caudad of the hepatic duct and drains the gall bladder.

Pancreas. This organ is long, narrow and lobulated. In color it is pale red. It is highly developed in birds and occupies the space between the two branches of the duodenum. Two or sometimes three ducts carry its secretion into the ascending arm of the duodenum near its extremity and just anterior to the hepatic duct.

\section{RESPIRATORY SYSTEMI}

The organs of respiration in birds differ markedly from those of mammals. They resemble more nearly the reptilian type of formation.

Air passages. The nostrils are represented by two narrow openings at the base of the upper mandible of the beak. The nasal chambers are short and narrow and are separated by a septum which is partly bony and partly cartilaginous. The turbinated bones of mammals are represented by three cartilaginous structures. The nasal cavities open into the mouth by a common, elongated slit in the median line of the hard palate. Cavities known as the suborbital or infraorbital sinuses occupy a position on either side of the head. They communicate with the nasal cavity by narrow passages which extend from a comparatively low level in the sinus, upward into the nasal cavity. The arrangement prevents natural drainage of the sinuses.

Birds possess an upper and a lower larynx. The former corresponds to the larynx of mammals but serves only as an opening for the passage of air. An epiglottis is absent but its function is 
assumed by two lateral lips which close the laryngeal opening during the passage of food through the pharynx. The inferior larynx, or syrinx, is the voice organ. It is located in the anterior thorax at the division of the trachea into two bronchi. The end of the trachea, in fowls, is compressed from side to side and forms the wall of the syrinx. In certain birds the syrinx is complicated in structure and may be formed of a number of tracheal rings expanded to form large bony cavities. Sound is produced by the vibration of membranes within the walls of the lower larynx.

The trachea is long in birds and its cartilaginous rings are complete. In the bronchi the cartilaginous rings are incomplete, a portion of the wall being formed by a membrane. The bronchi enter the lungs at the anterior extremities, become membranous and give off branches to these organs and to the air cells of the thorax and abdomen.

The lungs are pale or bright red in color and of a spongy texture. They are closely applied to the dorsal surface of the thorax and present several deep indentations into which the ribs fit. The ventral surface is smooth and is covered by a fold of the diaphragm. Only a small portion of the thoracic cavity is occupied by the lungs.

Air sacs. These are membranous receptacles into which the air is carried by terminal branches of the bronchi. Through these branches the cells are in direct communication with the lungs. They also, with certain exceptions, communicate with canals or spaces in the various bones, to which they supply air. The membranous wall is composed of two delicate layers, an external serous and an internal mucous layer. The latter is a continuation of the mucosa of the bronchi, the former is regarded as a reflection of the peritoneum and pleura. Nine air sacs are found in the thoracic and abdominal cavities. The single anterior thoracic air sac surrounds the inferior larynx, bronchi and large blood vessels of the region. It receives air from the anterior part of the lungs by two openings and communicates with deep seated air cells of the neck and with the clavicle, coracoid, scapula and sternum. Two lateral thoracic sacs receive air from the internal edge of each lung opposite the base of the heart. They transmit air currents to the air cells of the axillary region, vertebre, ribs and humerus. The diaphragmatic air cells are four in number, two anterior and two posterior. They are inclosed between the two diaphragmatic folds. Air is received from the lungs but is not communicated to bony structures from these. Two abdominal air sacs each receive a long voluminous bron- 
chial branch which leaves the respective lung on its posterior inferior surface. These sacs extend from the lungs to the cloaca and are the largest in the body. They supply the bones of the pelvic region and femur in those species in which these bones are aeriferous. The femur of the pigeon and the humerus of the ostrich have no air spaces but retain their marrow.

\section{URINARY SYSTEM}

The urinary tract is composed of two kidneys each drained by a ureter lying along its ventral face and proceeding caudad to empty into the cloaca posterior to the terminus of the rectum. The kidneys are elongated, reddish brown bodies divided into three principal lobes. They are closely applied to cavities in the pelvis on each side of the spinal column. These organs are not divided into macroscopically visible cortical and medullary zones as in mammals but have a homogeneous structure. In texture they are quite soft and rupture easily when pressed by the finger. The ureter does not originate in a renal pelvis but is formed by the union of several uriniferous tubules along the ventral face of the kidney. Its course may be traced by the white color of the urates which it carries, a material corresponding to the urine of mammals.

\section{GENERATIVE SYSTEM}

The male generative organs consist of two testicles each provided with a vas deferens or excretory duct. The testicles are oval shaped organs situated in the sub-lumbar region at the anterior extremities of the kidneys. They vary greatly in size at different seasons, but show a remarkable development during the breeding season. The two vasa deferentix pass along the kidneys by the side of the ureters, exhibiting transverse convolutions along their course. Each opens on a small papilla situated in the uro-genital division of the cloaca. The base of each papilla is surrounded by a plexus of blood vessels which serve the purpose of an erectile tissue during coitus. In the drake and gander a long single penis is developed.

The female generative organs consist of a single ovary and an oviduct situated on the left side of the abdominal cavity. The right ovary and oriduct usually fail to develop. Occasionally a rudimentary right oviduct is present. In one actively laying hen Gallagher observed a right oviduct which was nearly as long as the 
left oviduot and equally voluminous. Its anterior extremity exhibited an infundibular membrane in contact with the ovary. Posteriorly it opened into the cloaca opposite the left oviduct. The appearance of its mucous membrane suggested that it was capable of functionating.

The ovary is placed in the left sub-lumbar region, opposed on its superior surface to the ribs. In fowls it contains from twelve to fifteen hundred germ cells in various stages of development from microscopic granules to fully formed yolks in functionating ovaries. The oviduct terminates in the urogenital division of the cloaca just anterior to the left ureter. It is divided into five regions, each of which has a specific function. The first region, the infundibulum, is a delicate membrane of a funnel-like form which receives the ovum. Succeeding this is the part secreting albumen followed by the isthmus which secretes the shell membrane. The uterus or fourth region forms the shell. The last region, the vagina, is short and is provided with a sphincter muscle at its entrance to the cloaca.

\section{SKIN AND ITS APPENDAGES}

The skin of birds is without sweat glands. There are some small glands in the exterior passage of the ear, which secrete an unctious fluid. There is also a protruding glandular organ located above the coccyx between the tail feathers and known as the uropygium or rump gland, an organ peculiar to birds. It is round or oval in form and is divided into two halves by a medial septum. The exit passage is through a teat-like cone which projects above the level of the skin. Two external orifices are observed in the gland of the duck. In hens the gland is about the size of a pea, in geese about as large as a hazel nut and in the swan about an inch and one half in length. Its function is to secrete an oily fluid for water-proofing and dressing the feathers. The secretion is taken up by the beak and spread over the plumage.

The feathers are complicated modifications of epidermic structure. Each feather is composed of a quill, a shaft and a vane. The quill is embedded in a feather follicle of the skin and extends outwardly to the point where the barbs of the vane arise. It has an opening at its base for the reception of the dermal papilla and another opening at its point of junction with the vane. The shaft continues the quill to the extremity of the feather. The rane consists of two rows of barbs which arise on opposite sides of the shaft. 
These barbs each carry two rows of barbules which interlock with those of adjoining barbs. The feather is elevated by a small muscle located in the skin at the root of the quill.

The comb and wattles of fowls and the caruncle of turkeys are developed from the skin.

\section{INCUBATING PERIODS}

The length of time necessary for hatching eggs by natural incubation shows some variation. The normal period for the common hen is 21 days but this period may be shortened to 19 or 20 days by warm weather and unusually constant brooding by the hen. On the other hand, if the eggs have been abandoned and allowed to cool for a time, hatching may be delayed for several days. These variations doubtless occur in the case of other birds. The incubation periods of various birds, subject to variations as explained are as follows.

\section{Table I.- Incubating Periods}

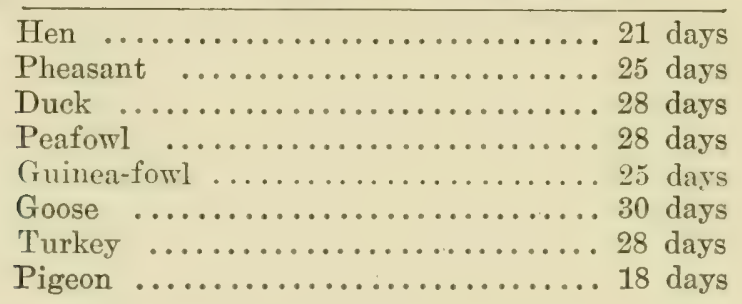

PULSE, TEMPERATURE AND RESPIRATION

Loer has made an extensive series of observations on the temperature, frequency of pulse and respiration in domestic birds. His results are summarized as follows.

Frequency of heart beat as determined by digital palpation after opening the thoracic cavity was 128 to 140 per minute in the hen and 141 to 149 per minute in the pigeon. The heart beat of the duck as determined by auscultation varied from 150 to 180 per minute. 
Table II.-Respiration Frequency and Norjal Temperatures of BIRDS

\begin{tabular}{|c|c|c|c|c|c|c|}
\hline \multirow{2}{*}{ Species } & \multicolumn{2}{|c|}{$\begin{array}{l}\text { Respiration } \\
\text { frequency }\end{array}$} & \multicolumn{2}{|c|}{$\begin{array}{c}\text { Centigrade } \\
\text { degrees }\end{array}$} & \multicolumn{2}{|c|}{$\begin{array}{c}\text { Fahrenheit } \\
\text { degrees }\end{array}$} \\
\hline & Min. & Max. & Min. & Max. & Min. & Max. \\
\hline Hen & 12 & 28 & 40.5 & 42.0 & 104.9 & 107.6 \\
\hline Turkey & 12 & 16 & 40.0 & 41.5 & 104.0 & 106.7 \\
\hline Pheasant & - & - & 41.0 & 44.0 & 105.8 & 111.2 \\
\hline Pigeon .... & 16 & 36 & 41.0 & 43.0 & 105.8 & 109.4 \\
\hline Duck ........... & 16 & 28 & 41.0 & 43.0 & 105.8 & 109.4 \\
\hline Goose .......... & 12 & 20 & 40.0 & 41.0 & 104.0 & 105.8 \\
\hline
\end{tabular}

\section{REFERENCES}

1. Boyce and Warrington. Observations on the anatomr, physiolngy and degenerations of the nervous system of the bird. Tr. Roy. Soc.. Tol. 191, 1899 .

\section{5 .}

2. Bradley. The structure of the forl. London: A. \& C. Black, Ltd.,

3. Burnett. Clinical pathology of the blood of domesticated animals. New York: The Macmillan Company, 1918.

4. Chauveau. Comparative anatomy of the domesticated animals. New York: D. Appleton and Company, 1905.

5. Ellenberger. Handbuch der vergleichenden mikroskopischen Anatomie der Haustiere. Erster Band. Berlin: Paul Parey, 1906.

6. Ellenberger u. Baum. Handbuch der vergleichenden Anatomie der Haustiere. Berlin: August Hirschwald, 1900.

7. Kaupp. The anatomy of the domestic forl. Philadelphia: W. B. Saunders Co.

8. Loer. Vergleichend phrsiologische Tntersuchungen iiber die normal Rektal temperature, Atem und Pulzfrequenz der Vogel. Inaug. Diss. Bern, 1909.

9. Owen. Comparative anatomy and physiology of rertebrates. Birds and mammals, Vol. II. London: Longmans Green \& Co., 1866.

10. Steen. Blutuntersuchungen bei gesunden Hühnern. Inang. Diss. Leipzig, 1913. 


\section{CHAPTER II \\ HYGIENE AND SANITATION \\ SECURING HEALTHY STOCK}

In establishing a flock of poultry it is highly important that precautions be taken to prevent the introduction of disease with the original stock. It is a common desire among beginners, to consider purchasing adult birds, in order to secure speedy returns. It should be borne in mind that the number of instances in which healthy young pullets may be purchased is comparatively rare. Too often hens are sold because they are old and past the best laying period, or because the flock has become unprofitable because of some disease. Fowl cholera, avian tuberculosis and diphtheria are commonly introduced by the purchase of adult stock.

Starting a flock from hatching eggs or day old chickens is the least dangerous method of introducing stock but there remains the danger of introducing white diarrhea.

\section{RELATION OF SOIL TO HEALTH}

Trell drained soil is desirable in that the presence of moisture and mud is minimized. A wet soil favors parasites. Least desirable for poultry yards is sticky clay or adobe. In wet weather the latter soil collects on the toes of chickens in the form of balls so large as to impede walking. Chickens kept on such soil require individual treatment to free the toes, and in doing this they are liable to be injured.

The continued use of a given area of soil for poultry, results in its becoming excessively infested with the eggs of various parasites and consequently a source of danger to the birds kept thereon. Therefore, it is highly desirable that chickens be not kept on the same soil year after year.

The problem of securing fresh areas for chickens is solved in various ways. Where the chicken house is a permanent, immovable structure, rards may be fenced off on either side and used in alternate years. When birds are kept under the colony system, the structures necessary for the fowls are built on skids. A team may 
be hitched to the building to move it as desired. A very desirable method of securing fresh soil is to move chicken houses in harmony with a crop rotation system. In this manner a given area is cropped for three or four years after chickens have occupied it, before the land is used for birds again. When quarters are so cramped that no change can be made in the chicken yards, deep plowing is of some advantage in turning up fresh earth and covering contaminated soil. Small areas may be sprinkled with lime, or be thoroughly soaked to some depth by the liberal application of a disinfectant.

\section{LOCATION AND CONSTRUCTION OF POULTRY HOUSES}

Dampness is very injurious to forvls, and consequently the buildings should be located with reference to securing good drainage after rains. Where choice is possible, sandy soil should be chosen on account of its permeability to water. Provision should be made to secure free ventilation without drafts of air. No especial effort need be made to secure warmth in a poultry house in the daytime even in a severe climate. The building should be so located as to permit the unobstructed illumination of the interior by sunlight.

The frequency with which ponltry houses must be cleaned and sprayed with parasiticides should be borne in mind when they are being constructed. Any structural detail which interferes with the free access to any part of a house by a man, should be avoided for it will interfere with routine cleaning. The habit displayed by mites, of collecting in cracks, should be recognized in providing buildings with a minimum of such places where vermin may collect. Nest boxes, perches and similar fittings should not be permanently attached to the building, but should be readily removable for cleaning and spraying.

Certain details of construction will at least constitute impediments to the dissemination of parasites and infective material from bird to bird. Thus, stretehing chicken wire under the roosts will largely prevent birds from coming in contact with droppings in the roosting quarters.

In houses designed for laying hens in a cold climate, each bird should be provided with from three to four square feet. Exercise, which is absolutely essential to health, will be provided by mixing some of the feed with clean litter on the floor. 


\section{CLEANING AND DISINFECTING BUILDINGS}

Previous to applying a disinfectant to a building it is essential that all dirt and filth be removed by sweeping, scraping or washing. This is necessary in order to expose the woodwork to the unobstructed action of the disinfectant.

A considerable range of choice of disinfectants is possible. Selection of a disinfectant will be made on the basis of relative cheapness, availability and suitability to the requirements imposed by the article to be disinfected.

Compound solution of cresol. This substance also known as liquor cresolis compositus, consists of a mixture of equal parts of cresol with a linseed oil-potash soap. It mixes well with soft water, but is less satisfactory in this respect when hard water is used. This disinfectant may be obtained at any drug store and on account of cheaper price is to be preferred to carbolic acid. It is employed for general disinfection in the proportion of four ounces to a gallon of water.

Formaldehyde gas. Formaldehyde is ordinarily obtainable in a forty per cent solution in water, a product which is known as formalin. Formaldehyde is a strong non-poisonous disinfectant, is not hindered in its action by albuminous material and in general is not harmful to metals. The gas is a good surface disinfectant and may be employed in disinfecting incubators and such rooms as may be easily made air tight. Ordinary poultry houses are not constructed tightly enough to warrant the trouble of sealing all cracks. Under conditions warranting its use, the room in which the gas is to be used is carefully examined and all cracks are sealed with strips of paper applied with paste.

The gas is liberated from the watery solution by mixing formalin with needle crystals of potassium permanganate. For each 1,000 cubic feet of air space there should be used 20 ounces of formalin and $16 \%$ ounces of potassium permanganate. The permanganate is placed in a shallow pan with flaring sides and the proper amount of formalin is poured in the pan. Provision should be made to protect the floor as the ingredients of the mixture may splash during the violent reaction that ensues. Splashing will be minimized by using a bucket, but in this case considerable formaldehyde gas may condense on the walls of the vessel. After mixing the chemicals, the operator should leave the room promptly to avoid inhaling the irritating fumes. The room should be kept sealed for eight hours. 
This method of fumigation is not applicable when the temperature falls below $65^{\circ} \mathrm{F}$. for the gas condenses under such conditions.

Disinfection may be accomplished by spraying objects with a mixture of 5 parts of formalin to 95 parts of water.

Carbolic acid or phenol. Pure carbolic acid exists at ordinary temperatures as needle-like crystals. This substance is frequently used in the form of liquefied carbolic acid which consists of 9 parts of the crystals mixed with 1 part of water. Carbolic acid is ordinarily employed as a disinfectant in a five per cent solution of the crystals. As the crystals dissolve slowly, solution is hastened by the use of hot water. The fluid may be applied liberally to surfaces as a spray, or fabries and implements may be immersed in the fluid for an hour. Carbolic acid is somewhat expensive, and on this account some other cheaper agent may be employed. The substance in question should not be confused with "crude carbolic acid" which is quite different in composition.

Crude carbolic acid. Commercial crude carbolic acid is a berproduct of the distillation of coal tar. It contains certain insoluble oils and a mixture of cresols and similar substances which possess disinfectant value. When the content of these is known, an effective disinfectant may be made by including these substances in two per cent solution. The disinfectant in question should not be confused with true carbolic acid (phenol).

Chloride of lime. Chloride of lime or chlorinated lime consists of slaked lime which has been exposed to the action of chlorine. It is a white powder which deteriorates in strength rapidly after opening the hermetically sealed container in which it is supplied. This disinfectant is particularly useful where the action of a deodorant is desired besides a disinfectant. It is commonly employed diluted with water in the proportion of six ounces to a gallon of water. The presence of organic matter interferes with the effectiveness of this agent.

Various coal-tar creosote disinfectants. Mixtures of rarious coal-tar products, principally creosote oil, with soap in the form of emulsions are on the market under a large variety of trade names. The disinfecting value of these products varies greatly, but when the carbolic acid coefficient is stated, one of these products may be used with assurance as to its efficiency. The expression carbolic acid coefficient, refers to the relative germicidal value of a product as compared with carbolic acid.

Bichloride of mercury or corrosive sublimate. This agent is 
usually employed in the strength of 1 part to 1000 parts of water. It is available in the form of tablets mixed with ammonium chloride to facilitate solution in water. A tablet may be added to a certain specified amount of water to make a disinfectant of a definite strength. Bichloride of mercury has the disadvantage of attacking metals and its activity is seriously impaired by contact with albuminoid substances. Its poisonous nature is some disadvantage.

Whitewash. Whitewash is made by adding about one pint of water to each two pounds of freshly burned lime. The vessel containing the mixture is covered and allowed to stand for about an hour while slaking occurs. Water is then added to the mixture until it is thinned to such a consistency that it may be readily applied.

Weather proof whitewash may be made as follows: Slake one bushel of quicklime in twelve gallons of hot water. Dissolve two pounds of common salt and one pound of zine sulphate in two gallons of boiling water. Pour this mixture into the slaked lime and add two gallons of skim milk, mixing thoroughly.

Whitewash has feeble disinfectant powers, but is useful in improving the illumination of interiors. To a certain extent it covers up infective material and fills up the smaller cracks which might harbor parasites.

The value of scattering air slaked lime about poultry houses is questioned in view of the irritation of the eyes and air passages induced by the dust.

Application of disinfectants. The frequency with which poultry houses are disinfected warrants investment in some form of pump. For the smaller establishments, a pump built to be used in a pail may suffice. In larger poultry farms a pressure spray pump mounted in a barrel on wheels forms a very convenient outfit.

CLEANLINESS OF FOOD AND DRINKING WATER

Feeding troughs may be provided with a cover of slats in the form of a peaked roof. Birds will be compelled to reach through the slats to eat mash, and cannot walk in the feed. Sanitary water fountains entirely prevent birds from walking in the drinking water. Further protection of the drinking water by the use of potassium permanganate is very generally practiced. A saturated solution of this substance is prepared in a large bottle or jar and is kept conveniently near the drinking fountain. When water is drawn for the birds, enough of the stock solution is added to impart a deep wine color to the water. 


\section{POST-MIORTEMI EXAMINATION}

The first step in making an autopsy on a bird is to examine carefully the exterior of the carcass for lesions of the diseases which exhibit external manifestations. The skin may show the presence of lice, mites or epithelial tumors. The head may be the seat of bird pox nodules, favus, or swelling due to roup. The skin of the head may present a pale appearance as in infectious leukemia, fowl typhoid, coccidiosis and tuberculosis or a congested or darkened appearance as in cholera, enteritis, pneumonia and infectious entero-hepatitis. Exudates of diphtheria may be observed in the mouth. Swellings of the joints of the wing or legs mar indicate tuberculosis or gout. Evidence of diarrhea should be looked for on the feathers surrounding the vent. The general condition of nourishment of the bird is determined by palpation, especially in the breast region.

In preparation for the examination of the internal organs it is best to pluck the feathers from the under surface of the body from the base of the neck to the vent and well up on each side of the body beneath the wings. The carcass is then stretched back downward over a table or board and held in position by cords attached to each leg and to the neck near the head. The other ends of the three cords are tied at the sides and one end of the table or board. Another method of holding the carcass in position is to nail each foot and the neck to the board. Those who have occasion to make frequent autopsies find it convenient to lay the bird out on a shallow, rectangular, flat bottomed tin pan fitted with eyelets on the sides and ends for the reception of cords tied to the feet and neck of the carcass. The pan serves to retain fluids, intestinal contents, etc., which may escape during the autopsy, and also is easily cleaned and disinfected.

The internal organs are exposed by making an incision through the skin and body wall on either side of the breast near the base of the wing and continuing it along the side to the vent and then forward to a point opposite the beginning of the incision. The ribs may be cut through with a knife at the cartilaginous articulation between the superior and inferior ribs or they may be severed by means of a pair of scissors or bone forceps. The breast bone and abdominal wall ean be removed by cutting through the hones of the shoulder girdle at the entrance to the chest with bone forceps. 
Peritoneal and other attachments are cleared amay with the knife as the inferior body wall is being removed.

Before removing any of the organs a general survey of the parts may reveal one of the following conditions: The presence of fluid in the abdominal cavity, which would be suggestive of peritonitis or enteritis; the presence of blood clots in either cavity indicating internal hemorrhage; yolk concretions or other egg material free in the abdominal eavity of females; tubercular masses on the intestine, liver or spleen; tumors in the various organs; or moldy growths in the air sacs, indicating aspergillosis.

The liver is then examined. It may be congested or show small necrotic spots in cholera and several bacillary septicemias or be pale and enlarged in infectious leukemia and fowl typhoid. In tuberculosis fat-like nodules with yellowish centers would be found. In entero-hepatitis the areas of necrosis would be yellowish or greenish. Various sorts of tumors may be present on the surface of the liver.

The alimentary tract requires a careful examination since many of the diseases are manifested by lesions at some point along its course. Frequently the congested areas of the mucosa may be detected through the serous membrane before the intestine is opened. It is advisable, however, to lay the whole tract open from the crop to the vent. The crop and proventriculus may show congested or inflamed areas as a result of the ingestion of strong irritants or poisons. The gizzard is relatively free of disease but may contain parasitic worms in its wall. The duodenum is often the seat of intense inflammation especially in the septicemic diseases such as cholera and fowl plague. Coccidia also cause congestion of the duodenal mucosa. In infectious entero-hepatitis and coccidiosis the ceca are usually distended with necrotic material and the cecal walls are ulcerated or desquamated. In enteritis the inflammation may involve a considerable portion of the intestinal mucosa and may be observed in any part of the small intestine or in the ceca or rectum. The cloaca is to be inspected for evidence of vent gleet. Intestinal worms may be present in any portion of the intestinal tract. Unless present in comparatively large numbers they should not receive special consideration. Round worms may be found gathered into large masses which tend to obstruct the passage of the contents of the intestine. When numerous they may also produce a catarrhal condition of the intestinal mucosa. Some tapeworms 
when numerous cause nodular formations on the intestinal wall which resemble the lesions of intestinal tuberculosis.

An enlarged, congested spleen may be associated with the acute bacterial septicemias. An enlarged, firm, mottled appearing spleen would suggest infectious leukemia. In the latter disease the kidneys are also enlarged and of firmer consistency. Tubereular nodules may be present.

A gangrenous or eystic ovary or an ovary exhibiting hard, angular, shrunken ova, in the case of the fowl, is practically diagnostic of B. pullorum infection. Occasionally gangrene of the orary may be due to some other microorganism. In such cases peritonitis is apt to be present.

The oviduct should be opened and examined for inflammatory changes, constrictions, tumors or the condition known as egg bound.

Normally the lungs are found deflated and closely applied to the breast wall. In acute congestion and pneumonia the lungs are distended and stand out in a firm position surrounding the heart. The pneumonic lung is solidified and will sink in water. Nodular growths in the lungs may represent tuberculosis or aspergillosis. The former is much less frequently found involving the lungs of birds than the abdominal organs. Aspergillar nodules are usually accompanied by moldy growths on the mucosa of the bronchioles or air sacs.

In young chickens the trachea and larger bronchi are slit open to discover the presence of the red worm, Syngamus trachealis, or of aspergillar growths.

Pericarditis, thickened pericardial fluid or hemorrhagic points on the heart indicate a septicemia such as cholera or fowl typhoid. The pericardium and also the liver may be covered by a fine white deposit consisting of urate of soda crystals in visceral gout, or as a result of disturbances of metabolism due to a diseased condition.

\section{REFERENCES}

1. Kaupp. Poultry diseases and their treatment. Chicago: American Journal of Veterinary Medicine, 1919.

2. Pearl, Surface and Curtis. Diseases of poultry. New York: The Macmillan Company, 1918. Co.

3. Salmon. Diseases of poultry. Washington: George E. Howard \&

4. Salmon. Important poultry diseases. Revised by Gallagher and Foster. U. S. Dep. Agr., Farmers' Bull. 95\%, 1918. 


\section{CHAPTER III}

\section{APOPLECTIFORM SEPTICEMIA AND SLEEPING DISEASE}

\section{APOPLECTIFORM SEPTICEMIA}

Characterization. The disease is a highly fatal septicemic infection of chickens caused by a streptococcus.

History. The disease was first described by Norgaard and Mohler in the United States and later was observed by Magnussen in Sweden.

Etiology. The causative organism is a streptococcus, the individual elements being .6 to .8 microns in diameter. There is great diversity in the length of chains. In tissues they are short and in cultures, long.

The organism is Gram positive, non-motile, and no capsule formation has been observed. It is an aerobe and a facultative anaerobe. Growth occurs on common solid and liquid media most abundantly at $37^{\circ} \mathrm{C}$., and less rapidly at room temperature.

In alkaline bouillon growth occurs within 24 hours at $37^{\circ} \mathrm{C}$. Long chains develop which form skeins or balls of floceulent appearance which are deposited on the sides and bottom of the tube. After three days the growth settles to the bottom in the form of a white non-viscid deposit. This upon agitation breaks up into numerous small particles.

After a sparse seeding on agar, growth is visible after 24 hours in the form of small shiny, grayish colonies about $1.5 \mathrm{~mm}$. in diameter. By transmitted light these appear to have a brown center surrounded by an irregular pale bluish border. The maximum size is attained on the third day. In agar stab cultures, minute spherical grayish white colonies with fimbriated borders, appear along the line of puncture. They do not coalesce, and very slight growth is observed on the surface.

The surface colonies on agar plates are very similar to those developing on a sparsely seeded slant culture. The colonies in the depths of the medium are seen as minute pearly points of granular appearance. With the aid of a hand lens the outline ap- 
pears ciliated. Similar but more profuse growth oceurs on glycerine agar, and serum-gelatin-agar.

In stab cultures on gelatin, growth is not observed until the fourth or fifth day. It consists of a finely granular line of minute spherical colonies, grayish white in color. The colonics have a fimbriated border and do not exceed a pinhead in size. The growth is not abundant nor characteristic and does not spread on the surface. No liquefaction occurs. Colonies on gelatin plates resemble those on agar, but are slightly more opalescent.

Growth on blood serum appears within 24 hours in the form of small whitish colonies .6 to $.8 \mathrm{~mm}$. in diameter. Sometimes the color has a yellowish tint. The colonies do not coalesce. The water of condensation becomes turbid by reason of the presence of small gray clusters of the streptococci.

Growth in milk occurs without causing visible change. In old cultures there is observed a solidification of the lower stratum, visible when the tube is turned upside down.

In litmus milk, the blue color is changed to a pale madder pink, indicating acid formation. No growth occurs on potato. No indol is formed.

Bouillon containing one per cent of glucose, lactose and saccharose, in fermentation tubes, forms a favorable medium. Growth is more profuse than in plain bouillon and extends into the closed arm. Gas is not produced in these sugars, but acid formation occurs. Similar results are obtained with galactose, maltose and the alcohols mannite, dulcite and sorbite.

No hemolytic effect oceurs in Conradi-Drigalski plate cultures. Acid formation occurs on litmus-lactose-agar plates.

Morbid anatomy. The skin displays hemorrhagic discoloration on the breast and neck, due to diffuse hemorrhages in the subcutaneous and muscular tissues. The abdominal cavity contains an abundance of sero-sanguinous exudate. Similar exudate sometimes is present in the pericardial sac. The reins of the mesentery are engorged. The liver is greatly enlarged, paler in color than normal and the entire surface is covered with a semi-organized plastic exudate. The spleen may be similarly swollen. The gall bladder is distended with bile, while the kidneys are hyperemic and swollen. The intestines, especially the duodenum, are observed from the peritoneal surface to be congested in patches. On opening the intestines, the mucosa corresponding to the discolored areas is considerably swollen. The contents of the intestine consist of 
blood stained feces and mucus. The lungs show circumscribed areas of congestion. On opening the cranium a profuse subdural exudate is observed. The meninges are injected and the ventricles contain an abnormal amount of discolored serum.

Symptoms. Information regarding symptoms is limited to observations made on the behavior of inoculated cases only. A short time after inoculation, birds display evidence of depression. There is staggering gait with effort to balance with the aid of the wings. If the birds are not disturbed they will lie in one place until death occurs in coma. Sometimes diarrhea occurs immediately before death.

Pathogenesis. Fowls are susceptible to intravenous or subcutaneous incculation and by the feeding of cultures. The mortality is heavy among fowls when the disease is spreading under natural conditions. Pigeons, rabbits and mice are susceptible. Intravenous inoculation of the duck may cause loss of coordination and death with endocarditis. Sparrows are more resistant than pigeons and chickens but succumb to large intramuscular and intraperitoneal doses. In the $\mathrm{dog}$, intravenous inoculation causes rise of temperature and lameness apparently due to a transitory arthritis. Abscesses may occur in the dog, a point of interest in that suppuration has not been observed in other animals. Guinea pigs, swine and sheep prove refractory. Cats may succumb to intraperitoneal inoculation. Horses exhibit a disturbance of health following intravenous inoculation.

Immunization. It is possible to immunize a fowl by intravenous inoculation of killed culture so that the bird will tolerate an otherwise fatal dose of virulent culture. Successive injections of virulent culture cause the blood serum to take on protective properties. Norgaard and MLohler have found this serum administered intravenously in a .5 c.c. dose, protective against a .1 c.c. intravenous inoculation of culture.

SLEEPING DISEASE

Synonym. Schlafkrankheit, Schlafsucht, (German); maladie du somneil, (French).

Characterization. The disease is a septicemic infection of fowls characterized by symptoms of sleepiness and caused by an encapsulated streptococcus.

History. The disease was first described by Dammann and 
Manegold in Germany and later by Greve in the same country.

Symptoms. The symptom most frequently occurring is a more or less marked desire to sleep. The hen sits for hours at a time with closed eyes and ruffled feathers, with the head bent backward in the feathers of the neck. At intervals the bird awakens and opens the beak wide as if panting. The conjunctiva, usually of one eye only, becomes reddened and markedly swollen. Secretion from the conjunctival sae dries on the edges of the lids. Except when the disease has a very short duration, the comb and wattles gradually become pale. Commonly diarrhea exists for several days and the bird dies in a highly emaciated condition. Birds may die suddenly after showing symptoms for only a day or less.

Morbid anatomy. The carcass exhibits the appearances common in hemorrhagic septicemia, such as distention of the subentaneous veins with dark red blood, infiltration of portions of the musculature with bloody, watery fluid. There is bloody, shiny, viscid fluid in the abdominal cavity and the peritoneum is swollen and diffusely reddened. The mucosa of the proventriculus and of the intestine is swollen while that of the latter is diffusely reddened. The liver is enlarged, brownish yellow in color and is friable in texture. It is studded irregularly with dark red hemorrhages. The spleen is enlarged and the capsule is distended while the pulp is fragile in texture. The kidneys are swollen and dirty yellow in color. The lungs are congested and permeated with numerous small, dark red hemorrhages and may be edematous. Fibrinous exudate may occur on the costal surfaces of the lungs. Punctiform hemorrhages may oceur under the epicarlium. The heart is distended with coagulated blood. The pericardium is often distended with pale serous fluid and a tough, fibrous layer of exudate frequently forms on the pericardium. Smears from the heart blood stained with carbol fuchsin are seen to contain chains of streptococei. The capsules are also seen distinctly enveloping single cocci as well as diplococci.

Etiology. The streptococcus causing the disease is designated Streptococcus capsulatus gallinarum. It differs in the length of the chains and in the size of the elements according to the sort of animal in which it appears as a natural disease or into which it is inoculated. These differences occur also in artificial cultivation. In animal blood and in fluid media, chains of up to 30 elements are observed and in sugar bouillon as many as 100 elements may necur. The size of the single elements varies between .3 and .5 micron. 
The individuals in the chains occur as diplococci. No motility is present.

The streptococci are stained by all of the common anilin dyes and are Gram positive. With Kühn's carbol methylene blue the capsules appear greenish in color. With a modification of Klett's anthrax capsule stain the cocci appear dark blue while the capsules are pale red or pink, with dark red undulating contours. The capsules are seen distinctly only in material from the animal body. The streptococci grown on the various culture media show them very poorly or not at all.

The organism grows aerobically and anaerobically and its optimum growth temperature is that of the body. It thrives best on solid blood serum, and grows well in milk. It can also be grown on meat extract bouillon, gelatin, agar, agar gelatin and grows least well on potato. In order to cause rich growth an addition of 4 to 6 per cent of glycerin or one per cent of sugar is necessary.

Acid is formed in saccharose bouillon and indol in limited amount. Gelatin is not liquefied.

It is very sensitive to drying and also to heat. In bouillon cultures which have been warmed to $80^{\circ} \mathrm{C}$., for five minutes it is killed. At $100^{\circ} \mathrm{C}$. it is killed in $1 / 2$ minute. One per cent solution of carbolic acid kills in 2 minutes, while a similar solution of liquor cresoli saponatus, lysol, and creolin renders it inactive in three minutes.

Pathogenesis. The disease is transmitted to hens by inoculation of blood or pieces of organs as well as by inoculation with culture. Infection with the latter succeeds through subcutaneous inoculation or when finely dissipated bouillon culture is inhaled. In carcasses of birds dead after subeutaneous inoculation, evidence of coagulation necrosis is seen frequently, accompanied by infiltration of the surrounding tissues with bloody or purulent fluid.

The time of appearance of symptoms in hens after artificial in. fection varies greatly. Sometimes sickness is noted after six days, with death occurring after seven days more. Again, it is possible for 30 to 70 days to elapse between the time of inoculation and death. The disease is transmissible by subeutaneous inoculation to pigeons, rabbits, white mice, gray mice, and lambs. The course of the disease is more acute in pigeons than in hens, but the lesions are similar. Dogs, ducks and guinea pigs do not succumb to artificial infection.

Differential diagnosis. The observation of capsules surround- 
ing the streptococei in smears from tissues will differentiate the affection from apoplectiform septicemia, a very similar infection.

Prevention. Measures similar to those recommended in the discussion of fowl cholera are indicated.

Treatment. No medicinal treatment is available.

\section{REFERENCES}

1. Dammann u. Manegold. Die Schlafkrankheit der Hühner. Deutsche tierärztl. Wchnschr., Bd. 13, 1905, S. 577.

2. Greve. Beitrag zur Kenntniss der Streptokokken-Krankheit (Schlafkrankheit) der Hühner. Deutsche tierärztl. Wchnschr., Bd. 16, 1908, S. 213.

3. Magnussen. Ueber Eine für Europa neue Hühnerseuche. Centralbl. f. Bakteriol. (Etc.), Orig., 1, Abt., Bd. 56, 1910, S. 411.

4. Norgaard and Mohler. Apoplectiform septicemia in chickens. U.S. Dep. Agr. Bureau Animal Indust. Bull. 36, 1902. 


\section{CHAPTER IV}

\section{FOWL CHOLERA}

Synonyms. Chicken cholera, pasteurellosis avium, cholera gallinarum, hemorrhagic septicemia of fowls; Hühnercholera, (German); choléra des poules, (French).

Characterization. Fowl cholera is an acute contagious septicemic disease affecting domesticated birds, which is accompanied by febrile temperature and causes heavy mortality. The popular designation "cholera" is frequently used to indicate any highly destructive disease of poultry.

Etiology. The causative organism is a member of the septicemia hemorrhagica group and has been designated by various names, among them, Bacillus avisepticus, B. bipolaris septicus, B. avicida, B. cholerce gallinarum, and Pasteurella avium. A strain isolated by Ward possessed the following characteristics:

"Morphology. The individual cells are short, non-motile rods, with rounded ends. They usually occur singly, but a few are seen in pairs. Spherical forms are numerous in actively growing cultures. The size varies from .4 to .6 micron broad and from 1 to 2 microns long. A bipolar arrangement of the protoplasm is demonstrated when carbol fuchsin and alkaline methylene blue stains are used. The bipolar staining is noticeable frequently in smear preparations from tissues. The presence of a capsule is suggested by an unstained area surrounding each organism when a background of stain is deposited upon the cover glass. The same appearance is noticeable in smear preparations from tissues. The organism retains the stain but faintly when treated after Gram's method.

"Biologic Characters. The organism is aerobic and facultative anaerobic. It grows readily at $37.5^{\circ} \mathrm{C}$, and with much less rapidity at room temperature.

"Agar. The colonies on one per cent agar, after forty-eight hours incubation at $37.5^{\circ} \mathrm{C}$, appear as round, smooth, thin, shiny disks, with entire border and measuring about $2 \mathrm{~mm}$. in diameter. Under a two-thirds obinctive they appear coarsely granular and show concentric circular markings. They appear smoky brown in color by directly transmitted light, and gray by reflected light. Colonies beneath the surface are usually lenticular in shape, and the granular appearance is more marked under a two-thirds objective than in the surface colonies. After twenty-four hours the growth on the agar slant culture is flat, smooth, shining, translucent, grayish white by reflected light, and smoky brown by transmitted light, with un- 
dulate border. The condensation water becomes decidedly turbid. After the first week of growth the liquid clears somewhat with the deposition of a viscous sediment. No pellicle has been observed on the condensation water.

"Glycerine Agar. Growth upon this medium presents no features distinguishable from that upon agar slant.

“Gelatin. Surface colonies, after about two weeks' growth at room temperature, are round, vitreous masses, with entire border and smooth shiny surface. A large proportion of the colonies are raised, forming a conical mass not exceeding $1 \mathrm{~mm}$. in diameter. Such colonies appear highly refractive by directly transmitted light. Under a two-thirds objective the colonies have a finely granular appearance and show concentric circular markings. Sub-surface colonies are lenticular in shape and granular.

"After three days' growth under similar conditions the growth in gelatin stab cultures is noticeable as a mass of closely aggregated colonies near the surface. After about two weeks the surface growth appears as a round, thin gray mass, with contoured surface and undulate border. At the same time the growth along the whole length of the path of the inoculating needle appears as a mass of closely aggregated distinct colonies.

"Potato. Implantations on this medium have not resulted in risible growth.

"Alkaline Bouillon. After forty-eight hours at $37.5^{\circ} \mathrm{C}$. the fluid becomes slightly clouded and does not clear up on standing, even after four months. In cultures two or three days old no sediment is deposited, but in older cultures a viscous sediment accumulates. The reaction is alkaline to litmus and markedly so in old cultures. No pellicle is formed, but occasionally a circular bluish band of growth adheres to the tube at the level of the surface of the fluid.

"Sugar-free Bouillon. Growth is similar to that in alkaline bouillon.

"Acid Bouillon. The growth is similar in appearance to that in alkaline, except that the turbidity is less marked and no accumulations have been noticed at either surface or bottom of liquid. Reaction becomes alkaline in old cultures.

" Mi?\%. No change occurs in this medium during the length of time that it is ordinarily kept under observation.

"Fermentation Tubes. (1) One per cent glucose bouillon: The liquid throughout the tube becomes uniformly slightly clouded in twenty-four hours at $37.5^{\circ} \mathrm{C}$, and remains so. The reaction becomes acid in two days. No gas is formed. A slight amount of viscous sediment collects.

" (2) One per cent lactose bouillon: The character of growth is similar to that in glucose. The reaction remains alkaline.

"(3) One per cent saccharose bouillon: The growth is similar to that of the two preceding. The reaction becomes acid in two days, but eventually becomes alkaline in cultures several weeks old.

"Dog Blood Serum. After twenty-four hours at $37.5^{\circ} \mathrm{C}$. the path of the needle is occupied by a smooth, shiny, raised growth of a color determined by that of the serum. The condensation water is markedly turbid. Six days later the growth, as well as the surface of a pellicle on the condensation water, has a coppery lustre. 
"Löfler's Blood Serum. After twenty-four hours' growth at $37.5^{\circ} \mathrm{C}$, the path of the needle is marked by a white, raised growth, with shiny, contoured or smooth surface and undulate border. The condensation liquid becomes decidedly turbid. After several days the surface of the growth becomes dull. The liquid is then observed to contain a viscous sediment and to have patches of pellicle floating upon the surface.

"Indol. A positive reaction is obtained in sugar-free bouillon cultures.

"Animal Inoculation. Subcutaneous or intravenous injection of fowls with 1 c.c. of a 24-hour bouillon culture resulted in death in about fifteen hours. Doses as small as 0.05 c.c. killed in about three days. A young rabbit inoculated with 0.5 c.c. in an ear vein was found dead fourteen hours later. A guinea pig inoculated with 1 c.c. subcutem was found dead in fourteen hours. Another receiving 0.5 c.c. subcutem survived thirty-six hours. A pigeon swallowing 1 c.c. died in twenty-one hours, and another receiving 0.12 c.c. subcutem was found dead fourteen hours later."

Pathogenicity. Besides common fowls, geese, ducks, turkeys, pheasants, pigeons and a large number of wild birds are susceptible to infection. Hemorrhagic septicemia in geese is discussed in Chapter XIX.

The virulence of the fowl cholera organism varies greatly. Hadley has shown that the minimum lethal dose for fowls may lie at any point between 10 c.c. and $0.000,000,000,000,001$ c.c. of a 48 hour bouillon culture. Hadley states that infection results from the inceulation into the breast muscle of less than fifty organisms and probably by the inoculation of no more than four. To test the infectiveness of the carcasses of fowls dead of the disease in a natural outbreak, Ward fed such material to ten healthy cockerels. On the first day following the exposure by feeding, three birds were dead; on the second day, three; on the third, fourth, fifth and sixth days, one bird each died. The incubation period may vary from eighteen hours to as many days.

The blood and all discharges of a sick bird are infective. Eggs have been demonstrated to contain the virus.

Infection may be induced in very small doses in a large variety of ways such as ingestion, subcutaneous, intravenous or intramuscular injection, introduction of culture into the conjunctival sac, or into the scarified skin.

Repeated passage of the virus through birds or through guinea pigs results in an increase of virulence of a strain. On the other hand diminution of virulence occurs under conditions not well understood. Doubtless there are also variations of virulence with respect to the species attacked during various outbreaks. 
In the case of infection by ingestion the organisms are believed to penetrate the minjured mucosa of the intestines and gain aceess to the lymph and thence to the blood. Death is undoubtedly caused by toxins. There is however, lack of unanimity of opinion as to whether the toxic effect is due to intracellular or to extracellular toxins.

Prognosis. Prognosis is serious, for 90 to 95 per cent of acute cases succumb.

Symptoms. In the peracute form, the bird either drops dead suddenly or more often dies on the roost at night.

In the acute form the first noticeable symptom in the fowl is the yellow color of the droppings. The yellow material consists of urates and is usually found on the feathers near the vent. Diarrhea follows later. The discharge varies considerably in color and consistency. It may be a greenish mass of pasty consistency, a brownish red mucus or a viscous transparent fluid. The yellow color of the urates is the most constant character.

Evidence of sickness is afforded by the unnatural attitude of the feathers and by disinclination of the bird to move about. During the later stages, no food is taken, but very sick fowls may drink copiously. Drowsiness is marked in the later stages. Frequently a mucous discharge drips from the mouth. The temperature varies from $109^{\circ}$ to $112^{\circ} \mathrm{F}$.

Death usually occurs within three days from exposure to infection but has been observed to occur within eighteen hours after ingestion of material from the carcass of another bird dead of the disease. During an acute outbreak, sickness is seldom noticed more than twenty-four hours before death.

The chronic form of the disease generally appears at the end of an outbreak. The birds are dull, depressed and show persistent or intermittent diarrhea. The mucosæ are pale and emaciation is marked. Arthritis may develop in one or more joints, and leads to ankylosis.

Morbid anatomy. A reddening of the skin of the breast and abdomen is frequently observed. The eomb often is dark red in color, but may be pale. There is a general congestion of the blood vessels of the visceral organs. The heart, in almost every case, is studded with punctiform hemorrhages. Less frequently are observerl a fibrinous or a gelatinous exudate in the pericardium. One of the lesions cecurring most frequently consists of hemorrhages in the first and second duodenal flexures. They may be so deep seated as to be 
visible from the peritoneal surface. Under such conditions the contents of the duodenum consist of a pasty mass permeated with blood clots. The contents of the intestines sometimes consist of a creamcolored pasty mass, or may be green or brownish-red in color. In many cases the liver is permeated with whitish punctiform areas of necrosis. The oral, nasal, and pharyngeal cavities frequently contain a viscid mucous fluid.

The lungs may show congestion and catarrhal or hemorrhagic pneumonia. More rarely caseous foci are present. Sero-fibrinous exudate may oceur in the air sacs.

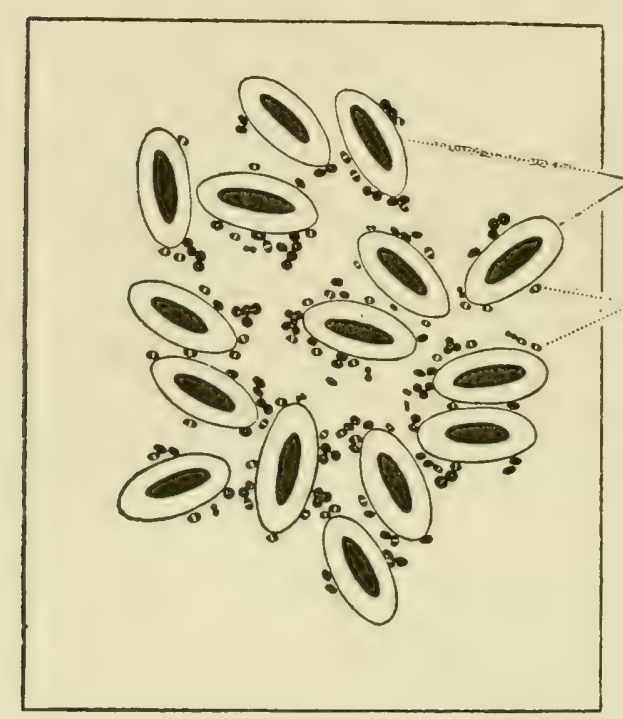

Fig. 3. Blood smear from blood of a pigeon infected with fowl cholern. a, red blood cells; b, fowl cholera bacteria. (Klee)

Hemorrhages are usually restricted to the heart and duodenum, and occur elsewhere in the intestines rarely. Still less frequently are they observed to be abundant throughout the $b$ skeletal muscles.

Microscopic examination of stained smears from heart blood and organs will reveal numerous bipolar staining rods.

Differential diagnosis. Avian plague can be differentiated by the fact that no organisms are present in the blood as demonstrated by cultures or by the microscopic examination of

smears. Further, inoculation of pigeons and rabbits with material from a case of plague will not produce infection in these animals.

Fowl cholera has features in common with fowl typhoid. However, diarrhea is not usual in the latter and at autopsy the intestines are found to be pale, and the contents are normal in consistency. In fowl typhoid the heart is pale and dotted with grayish points due to cell infiltration. A bacteriological examination is necessary for differentiating the various cholera-like septicemias. This is not necessary in practice for no information is available to 
warrant handling such ontbreaks by methods other than those used to combat cholera unless biologic products are used.

Treatment. The treatment of individual sick birds is not recommended, and their immediate slaughter is advised.

Immunization. Pasteur in his memorable studies on immunization against disease first worked with the fowl cholera organism in an attempt to produce an immunity against the disease caused by this organism. He utilized in his experiments cultures of the fowl cholera bacterium which he believed had become attenuated by exposure to air for a period of several months. His favorable results laid the foundation for the further study of the control of disease by the utilization of the specific causative organism as an ageney of prevention. Kitt, Cogny, Vages and others working along the lines pursued by Pasteur have failed to substantiate his findings. However, it is quite possible that Pasteur worked with a strain of the fowl cholera oreanism which had immunizing value. Such an inference may be drawn through the researches of Hadley who demonstrated that a particular strain of the fowl cholera organism designated strain 52, although practically arirulent, had the power, when injected in the live state, of conferring immunity to rabbits against the subsequent injection of a highly virulent strain. These findings were, to a considerable degree, confirmed by Gallagher who also found that fowls acquired a marked resistance to comparatively large amounts of a virulent culture following the injection of immunizing strain 52. At the same time the latter's experiments with killed cultures of several strains of the fowl cholera organism, including strain 52, as immunizing agents gave negative results. In each case fowls injected with bouillon cultures of organisms killed by heating at $60^{\circ} \mathrm{C}$. for 1 hour, by carbolizing to .5 per cent or by mixing with ether in equal parts, failed to show resistance to a subsequent injection of $.000,000,001$ c.c. of a virulent strain.

Mack failed to produce immunity by the use of killed organisms against artificial infection but claims successful results in a number of natural outbreaks. The use of an avirulent living organism with immunizing properties offers the most promising methon of prevention. One c.c. of a 24 to 48 hour bouillon culture grotrn at $37^{\circ} \mathrm{C}$. is injected subeutaneously. The point most convenient for injection is the unfeathered area of the breast beneath the wing.

It is possible to produce an antiserum by injecting a horse re- 
peatedly with cultures of the fowl cholera organism. The passive immunity induced by its injection into fowls is short, and its usefulness is limited to flocks already infected or in grave danger of becoming so.

Immunization with an aggressin has been accomplished in an experimental way by Weil. He produces a pleural exudate in a rabbit by injecting with fowl cholera bacilli. This exudate is carbolized and heated for three hours at $44^{\circ}$ C. A dose of 5 c.c. is said to immunize against subsequent injection of a lethal dose.

Prevention. Sanitation with reference to known methods of dissemination should be the basis of combating the disease. The chief sources of infection within an infected flock are dead birds eaten by members of the flock, and contamination of food and water by droppings. On large poultry farms, measures to secure isolation of the center of infection should be enforced promptly. This may involve temporary fencing, or moving the buildings, if portable.

Daily inspection of the roosting houses at daybreak should be made to remove dead birds and to secure sick ones before they have opportunity to go afield. Constant watch during the day will reveal droopy birds which should be killed.

Feeding troughs may be so arranged that the birds can only put in the head and do not have opportunity to contaminate the feed with their feet. The drinking water may be rendered safe by adding to it mercuric chloride in the proportion of one part to six thousand parts of water. Earthenware containers should be used.

Disinfection of houses and adjacent areas should be carried ont daily. Since the floor of the roosting house is highly contaminated by droppings, it should be disinfected if possible, before the birds leave the roost. At any rate, the birds should be excluded from the roosting house until it is possible to disinfect.

Poultrymen, familiar with the ravages of cholera, have been known to ship sick and exposed birds to market, as the simplest way to avoid financial loss. Apart from the obvious effect in removing a center of infection, it has another effect in further spreading infection. Birds dying during the trip to the shipping point are thrown by the roadside, where they are liable to be partially eaten by any fowls encountering them. The disease may be introduced into a flock in a large number of ways such as introducing birds in the incubation period of the disease, the carrying in of infection on the shoes, by water, by animals, by pigeons or wild 
birds. All these possibilities, except perhaps the last two, suggest appropriate defensive measures.

\section{EDEMA OF THE WATTLES OF FOWLS}

Characterization. Edema of the wattles is an infectious disease involving the wattles and which usually runs a chronic course.

History. The disease has been deseribed by Seddon who observed it among fowls in the suburbs of Melbourne, Australia.

Etiology. From all acute cases it is possible to isolate a short rod indistinguishable from the one causing fowl cholera. In view of this fact and since fowl cholera is very common in the region in question it is likely that the wattle lesion merely represents a localized chronic form of disease caused by the fowl cholera bacterium.

Pathogenicity. Scarification of the wattle of fowls and rubbing in of culture in one case resulted in general illness, conjunctivitis, edema of wattle, diarrhea and formation of necrotic material. The bird when killed showed little change beyond the head lesion which was found to contain the organism that was inoculated. Another fowl inoculated in the same way showed edema and necrosis at the point of inoculation. The bird subsequently displayed immunity to infection through scarification or by subcutaneous injection of virulent culture. A hen receiving one fourth c.c. of culture intramuscularly died in 24 hours and showed septicemic lesions at autopsy.

The disease occurred spontaneously among White Leghorns possessing large wattles. These brushed on the ground during feeding, which offered an opportunity for injury and infection. Wounds received during fighting offer another opportunity for entrance of virus.

The pigeon is killed by intramuscular inoculation of culture. Rabbits succumb to the infection with lesions of hemorrhagic septicemia.

Symptoms. There is sudden enlargement of the wattles due to more or less distention with fluid. In the beginning stage the wattles are hot, dark red in color and upon incision a clear fluid exudes. The birds manifest symptoms of general illness, loss of appetite, ete. Sometimes there is conjunctivitis. Subsequently there is gradual absorption of fluid accompanied by thickening of the wattle by fibrous tissue. Nodules sometimes form in the tissues beneath the 
mandible or under the scars of wounds acquired during fighting. The morbid process leads to disfigurement of the wattles by swellings and by their assuming a crinkly form. In a small percentage of cases of the disease fatal septicemia occurs.

Morbid anatomy. The more highly thickened areas in the wattles consist of masses of caseous material. In some cases the necrotic mass is surrounded by fibrous tissue, yellowish brown in color and hard, almost horny in texture.

Treatment. Cropping the wattles offers the most satisfactory means for dealing with the infection. The operation is described on page 298 .

Prophylaxis. Preventive measures must be essentially those employed against fowl cholera.

\section{CHOLERA-LIKE SEPTICEMIAS}

The literature contains descriptions of a considerable number of acute infections occurring among fowls, turkeys, geese, ducks, swans and other birds. In a number of instances the diseases have been shown not to be fowl cholera by bacteriological evidence that is convincing today. In other instances the features differentiating the disease from fowl cholera do not appear convincing at the present time. The reports cover a considerable period of time during which many changes have occurred in methods of identifying cultures. Thus the old bacteriological descriptions are inadequate for making an accurate classification of these infections.

There is little practical need for a close examination of the etiology of these septicemias. The general methods of prophylaxis are identical with those necessary in combating fowl cholera.

\section{DISEASES IDENTICAL WITH FOWL CHOLERA}

There are examples of diseases substantially identical with fowl cholera which have been described under different names or without assignment of name. Lisi described a fatal septicemia occurring in fowls which caused the death of all the birds in the flocks inraded. Tocard and Leclainche agree with Lignières that the disease was fowl cholera. Rabieaux observed a disease which he designates as a hemorrhagic septicemia of the duck and fowl. Lignières as well as Hutyra and Marek agree that Rabieaux does not show that the disease described by him is different from fowl cholera. 
Lucet describes a septicemic disease of fowls and turkeys. He differentiates the disease from fowl cholera by the insusceptibility of the rabbit to subcutaneous inoculation and other peculiarities even less convincing.

Etiology. The organism causing the infection is a short, slightly motile rod which does not stain by the Gram method. It does not grow on potato and gelatin is not liquefied.

Pathogenicity. The disease is inoculable from fowl to fowl, from the fowl to the turkey, from turkey to turkey and reciprocally. It is also transmissible between the two species by ingestion. The pigeon is insusceptible to subcutaneous inoculation. The guinea pig is not affected by subcutaneous or intraperitoneal injection. The rabbit is insusceptible to injections in the same way, but succumbs to intravenous inoculation.

Symptoms and lesions. There is nothing distinctive in the symptoms displayed while the lesions are those of fowl cholera.

\section{EPIZOOTIC PNEUMO-PERICARDITIS IN THE TURKEY}

Characterization. The disease is an infection of the turkey characterized by lesions of the lung and pericardium.

History. M'Fadyean first described the infection in England, while Jowett encountered it near Cape Town.

Etiology. The organism causing the disease is a small ovoid rod closely resembling that causing fowl cholera. M'Fadyean notes that it possesses motility, while Jowett is silent on the point. It is Gram negative and is readily stained by the ordinary dyes. No liquefaction is produced in gelatin, no gas is formed in agar shake cultures, no acid formation nor coagulation occurs in milk and no visible growth is observed on potato. Growth occurs equally well under aerobic or anaerobic conditions. No unusual or striking characteristics are presented by the growth on agar slant or bouillon cultures.

Pathogenicity. In the natural outbreak observed by Jowett, turkeys only succumbed, while fowls, geese and pigeons in close contact with them escaped infection. Subcutaneous inoculation of the turkey with cultures caused symptoms of dullness, stiffiness and mouth breathing followed by death in a few days. Guinea pigs and rabbits succumb to inoculation. Fowls are only slightly susceptible while pigeons sucumb to intraperitoncal inoculation. 
White rats die after intraperitoneal inoculation, but subcutaneous inoculation may fail.

Morbid anatomy. Severe pericarditis is one of the most noticeable characteristics observed at autopsy. The pericardium may show extensive adhesions to the heart wall, and the latter may be covered with a layer of yellow colored fibrinous exudate. The pericardial sac may contain sero-fibrinous exudate. The lungs show pneumonic changes and are frequently completely hepatized. Beyond possible fatty changes in the liver and paleness and swelling of the kidneys, changes in other organs are not marked.

Microscopic examination of blood smears from heart blood, pericardial exudate, lung and various organs, reveals the presence of a bipolar rod.

Relation of disease to fowl cholera. M'Fadyean believes that the effects produced on various animals by the organism described by him, warrant the differentiation of the disease from fowl cholera. He notes that pericarditis and pneumonia oceur in fowl cholera, but not with such constancy as they occur in the disease described by him.

\section{AN EPIZOOTIC AMONG FOWLS}

Mazza reported upon a disease that caused heavy mortality in various parts of upper Italy.

Etiology. Cultures from various organs almost always yield growth of a motile rod, while culture media seeded from exudate, blood and brain are more often sterile. Mazza concludes that the organism differs from that of fowl cholera in several particulars. It is larger than $B$. avisepticus, does not usually exhibit bipolar staining, is motile, is not very pathogenic for rabbits and is rarely observed in the blood.

Pathogenicity. Hens and pigeons succumb to the infection without exception. Guinea pigs are completely insusceptible while rabbits are very slightly susceptible.

Symptoms. In general the hens die suddenly during the night without having previously shown distinct symptoms of disease. In other cases, symptoms like those occurring in fowl cholera are observed.

Morbid anatomy. In external appearance the fowls usually show no signs of emaciation. The only abnormal conditions encountered are darkening of the comb and large red areas on the skin of the abdomen and of the breast. The subcutaneous cellular tissue 
appears scanty and oceasionally hemorrhages are observed in this locality. Quite constant lesions are encountered in the chest and involve one or both lungs. There is a serous exudate in the chest cavity sometimes with other signs of inflammation. MLore rarely there is observed serous bloody fluid in the pericardium and reddening of the mucosa of the duodenum. In the cranial cavity there is rarely observed a reddening of the pia mater and of the brain substance.

HEMIORHAGIC SEPTICEMIA OF THE RING DOVE

Leclainche reports the occurrence of a septicemic disease of ring doves from which he isolated an organism regarded as belonging to the hemorrhagie septicemia group.

Etiology. The causative organism is an ovoid rod identical in shape with that causing fowl cholera, but somewhat larger. The organism shows bipolar staining and is Gram negative. Growth occurs on potato and gelatin is not liquefied.

Pathogenicity. The infection is transmissible to the ring dove by inoculation and by ingestion. The domestic pigeon is much more resistant. The fowl is absolutely refractory while the rabbit and guinea pig are susceptible.

Symptoms. The symptoms are those usually presented by birds affected with a septicemia.

Morbid anatomy. Lesions consist chicfly of hemorrhagic inflammation of the small intestine.

\section{CHOLERA OF COSCOROBA SWANS}

Tretrop observed a septicemia among swans in the zoological garden of Antwerp to which he has given the above designation. The species involved was Coscoroba candida. About fifty birds were in the flock, of which the majority died. A large number of other species of swans and other waterfowl were in contact with the diseased ones but escaped infection.

Etiology. There is found constantly in the organs of the diseased birds, a microorganism designated Bacillus coscorobr. It is an ovoid motile rod 1.5 to 2.5 microns long and 1 to 1.4 microns broad. Bipolar staining is observed and the organism is Gram negative. The organism grows equally well in bouillon, milk, gel- 
atin and not on potato. Milk is coagulated and indol formed. Gelatin is not liquefied.

Pathogenicity. The mouse is very susceptible to inoculation but the white mouse will not contract the infection by ingestion. Birds of the order passeres succumb rapidly to intramuscular injection of culture. The duck and fowl are insusceptible. Numerous species of swans, geese and ducks in contact with the diseased birds, escaped infection.

Symptoms. The birds remain in a squatting position, and show the usual manifestations of sickness exhibited by those affected with a septicemia.

Morbid anatomy. The subjects are generally in good condition. The muscles offer nothing special and the heart appears normal. Commonly, active congestion is observed in the lungs. Sometimes one or two caseous foci are present, which are easily enucleated. The liver is enlarged, dark in color and frequently shows small whitish spots. The spleen is sometimes slightly engorged, sometimes slightly increased in size. The intestine always shows diarrhea with contents yellowish or yellowish green in color. The intestines do not show marked injection of the blood vessels. Nodules the size of a small pea are frequently found in the abdominal cavity in the vicinity of the vertebral column.

\section{HEMIORRHAGIC SEPTICEMIA OF THE SWAN}

Fiorentini observed a septicemic infection among swans in the zoological gardens of Milan.

Morbid anatomy. In young birds that have died quickly there is an edematous infiltration of the lungs with ecchymoses on the serous membranes. There is also a slight hyperemia of the intestinal mucosa, dark blood clots in the heart cavity and cloudy degeneration of the liver cells. In older birds that have died after several days sickness there are more severe lesions. The lungs show fibrous pneumonia in the stage of gray hepatization. The upper lobes of the liver show a grayish yellow exudate composed mostly of lymphoid elements, besides marked thickening of Glisson's capsule. The hepatic tissue shows a marked infiltration with red blood cells in the spaces between the liver cells. In numerous places in the liver there are yellowish zones caused by the fatty degeneration of the cellular elements. In the intestine there is slight hyperemia but no exudate nor swelling. On the serous membranes there are numer- 
ous confluent ecchymoses, especially along the course of the coronary arteries.

Etiology. The blood of dead swans yields cultures of a short rod with rounded ends and which shows bipolar staining. The shape of the organism is very similar to that of the fowl cholera bacterium, but it is somewhat larger. The organism has some characteristics in common with that of fowl cholera. Both stain alike by the Gram method and do not give an indol reaction. However, they differ in other characteristics as the swan organism is motile. It also forms rods as long as 4 microns. Growth in bouillon, agar and gelatin furnishes no distinguishing characteristic. Copious brown colored growth occurs on potato accompanied by a bad odor.

Pathogenicity. The organism is virulent for the swan and a goose (Anser aegyptiacus) while various aquatic and other birds escaped infection.

Rabbits, guinea pigs, ducks, geese, hens and pigeons succumb to intramuscular inoculation of culture.

\section{INFECTIOUS ENTERITIS OF PHEASANTS}

Fiorontini observed an epizootic among pheasants in the public gardens of Milan which occurred a short time after an epizootic of the same nature among swans. The disease was slightly contagious for after many months the greater part of the birds escaped infection.

Etiology. The organism causing the disease resembles that of fowl cholera. It measures .7 micron in diameter and 1 to 2 microns in length. In cultures the organism forms chains which are sometimes very long: Growth in bouillon is rapid at $22^{\circ}$ to $24^{\circ} \mathrm{C}$. Indol is produced. On gelatin in $t 8$ hours, a gray layer of growth develops. On potato the color varies according to the development of the growth. After 48 hours, the color is pale yellow, which changes to brown while the borders are rose brown. The culture has the appearance of honey.

Pathogenicity. Pheasants alone are attacked and a preference for males is exhibited. The infection is transmitted by inoculation of cultures subcutaneously or intravenously in small amounts. Ingestion of culture induces disease identical with that occurring naturally. Hens are not affected by receiving 2 to 3 c.c. of virulent culture and such inoculation does not assure immunity to fowl cholera. Rabbits and guinea pigs are immune. 
Symptoms. The birds display depression and the gait is uncertain. The appetite is poor. Late in the disease an abundant diarrhea occurs, with the passage of grayish yellow or greenish colored material. The state of coma and somnolence seen in fowl cholera does not occur. The attack lasts five to eight days.

Morbid anatomy. Lesions occur throughout the intestine. The mucosa is congested, is reddish brown in color and is covered with a viscid layer or by a croupous exudate. Ecchymoses are not found on the serous membranes. The liver is friable and the spleen is swollen but not hemorrhagic.

\section{REFERENCES}

1. Fiorentini. Enterite infettiva dei fagiani. Atti della Società italiana di scenze naturali, 1896, p. 89.

2. Fiorentini. Hämorrhagische Septikämie der Schwane. Centralbl. $f$. Bakteriol. (Etc.), 1. Abt. Orig., Bd. 19, 1896, S. 932.

3. Gallagher. Fowl cholera and other hemorrhagic septicemia immunization experiments. J. Am. Vet. M. Ass., Vol. 50, (n.s. Vol. 3), 1917, p. 708 .

4. Hadley. Fowl cholera and methods of combating it. Rhode Island Agr. Exp. Sta. Bull. 144, 1910.

5. Hadley. A biological study of eleven pathogenic organisms from cholera like diseases in poultry. Rhode Island Agr. Exp. Sta. Bull. 146, 1910.

6. Higgins. Notes upon an epidemic of fowl cholera and upon the comparative production of acid by allied bacteria. Jour. Exper. Med., Vol. 3, 1898 , p. 651.

7. Jowett. Epizootic pneumo-pericarditis in the turkey. J. Comp. Path. and Therap., Vol. 21, 1908, p. 324.

8. Kitt. Die Serumimpfung gegen Geflügelcholera. Monatshefte $f$. prakt. Tierh., Bd. 16, 1904, S. 1.

9. Leclainche. Sur une nouvelle septicémie hemorrhagique. La maladie des palombes. Ann. de l’ Inst. Pasteur, T. 8, 1894, p. 490.

10. Lucet. Dysenterie épizootique des poules et des dindes. Ann. de $l$ ' Inst. Pasteur, T. 5, 1891, p. 312.

11. Mazza. Bakteriologische Untersuchungen iiber eine nenerdings auf getretene Hühnerepizootie. Centralbl. f. Bakteriol. (Etc), 1. Abt. Orig., Bd. 26, 1899, S. 181.

12. Mack and Records. The use of bacterins in the control of fowl cholera. Univ. Agr. Exp. Sta. Bull. 85, 1916.

13. M'Fadyean. Epizootic pneumo-pericarditis in the turkey. J. Comp. Path. and Therap., Vol. 6, 1893, p. 334.

14. Nocard and Leclainche. Les maladies microbiennes des animaux. Paris: Masson et Cie. 1903.

15. Pasteur. De l' attenuation du virus du choléra des poules. Compt. Rend. Acad. Sci. Paris, T. 91, 1880, p. 673. 
16. Pasteur. Sur les maladies virulentes, et en particular sur la maladie appelée vulgairement choléra des poules. Compt. Rend. Acad. Sci. Paris, T. 90,1880 , p. 239.

17. Rabieaux. Sur une septicémie hémorrhagique du canard et de la poule. J. de méd. vét. et de zootech., T. 51, 1900, p. 129.

18. Seddon. Oedema of the wattles of fowls due to an organism of the pasteurella group. Vet. J., Vol. 21, 1914, p. 24.

19. Seddon. A diseases of the wattles of the fowl. J. Agr. Victoria, Vol. 12, 1914, p. 426.

20. Tretrop. La maladie des cygnes coscoroba. Ann. de l' Inst. Pasteur, T. 14, 1900, p. 224.

21. Ward. Fowl cholera. Univ. of Cal. Agr. Exp. Sta, Bull. 156, 1904. 


\section{CHAPTER V \\ FOWL TYPHOID AND SIMILAR INFECTIONS}

FOWL TYPHOID

Synonym. Hühner typhus (German).

Characterization. Fowl typhoid is a specific infectious disease of fowls, having the general characteristics of a septicemia and is caused by Bacterium sanguinarium (Bacillus gallinarum).

History. The disease seems to have been first described by Klein in England in 1889 under the name of infectious enteritis of fowls. The organism was designated by him, Bacillus gallinarum. Hadley states that a culture of this organism has been preserved since its isolation by Klein, and that a study recently made by him reveals the organism to be identical with Bacterium sanguinarium. The disease and its causative agent were described by Moore in 1895. Other outbreaks in the United States have been described by Dawson, Curtice and by Taylor.

Pfeiler and Rehse observed the same disease in 1912, gave it the name "Hühner typhus," and described the organism under the name Bacterium typhi gallinanum alcalifaciens. A second outbreak occurring in 1915 has been described by Pfeiler and Roepke.

Etiology. The characteristics of the organism are described by Moore as follows:

"Morphology. Bacterium sanguinarium varies somewhat in size according to the medium in which it has developed. In tissues of fowls or rabbits it is from 1.2 to 1.8 microns long and from 1 to 1.3 microns broad. The ends are tapering or rounded in cultures; in the short forms it could easily be mistaken for a micrococcus. In tissue it frequently appears in small clumps, but usually in pairs united end to end. Spores or vacuoles have not been discovered. Involution forms are common. In eultures on agar it is more slender than in tissues. When examined in a hanging drop preparation, especially at the edge, it frequently shows a marked polar arrangement of the cellular protoplasm. In these preparations there is observed a marked dancing motion of the organism. In old bouillon cultures short chains composed of these organisms united end to end are sometimes observed.

"Staining. It stains with the aniline dyes ordinarily used, but retains the coloring matter very feebly, or not at all, when treated after the Gram method. 
"Cultivation. This organism is readily cultivated on the ordinary media. It is obtained in pure cultures from the heart blood or liver of a fowl just dead from the disease.

"Agar. On this medium, at $37^{\circ} \mathrm{C}$, the growth is moderately vigorous. It has a grayish glistening appearance. Isolated colonies are from 1 to $2 \mathrm{~mm}$. in diameter, convex, and with sharply defined borders. Agar plates emit a peculiar penetrating odor, which differs decidedly from the pungent odor given off by Bacterium suisepticum. The growth on this medium resembles very closely that of $B$. suipestifer.

"Gelatin. In this medium the growth is less vigorous. In stick cultures it is more abundant along the line of inoculation than on the surface. Isolated colonies are about $0.25 \mathrm{~mm}$. in diameter, appearing to the unaided eye as homogeneous bodies, but slightly granular under low magnification. On the surface of the gelatin the colonies are granular and slightly spreading. They are not characterized by any distinctive markings. There is no liquefaction or softening of the medium.

"Potato. On the surface of potatoes a delicate grayish yellow growth appears after forty-eight hours when kept at a temperature of $35^{\circ} \mathrm{C}$. Frequently there is no development, owing, presumably, to the acids in the potato.

"Bouillon. In alkaline peptone bouillon at $36^{\circ} \mathrm{C}$. the growth imparts a uniform cloudiness to the liquid within twenty-four hours. If the bouillon contains much sugar the reaction becomes acid, otherwise it remains alkaline. A grayish friable sediment forms in the bottom of the tube. After several days' standing the growth settles, leaving a clear supernatant fluid. In a simple peptone solution containing one-half of 1 per cent sodium chloride the growth is less vigorous than in the one containing the meat juice. In meat extract bouillon the growth is likewise feeble. In acid peptone bouillon there is a very faint cloudiness imparted to the liquid.

"Alkaline bouillon containing 1 per cent dextrose in the fermentation tube becomes cloudy within twenty-four hours after inoculating and strongly acid in reaction. Similar tubes of bouillon containing saccharose and lactose become clouded throughout but they remain alkaline in reaction. The degree of alkalinity increases with age. Gas is not produced during the growth in bouillon containing these sugars.

"Life conditions and properties. This organism develops at a temnerature from 20 to $41^{\circ} \mathrm{C}$. It does not grow well in acid media. It produces indol.

"Resistance. It is destroyed at $50^{\circ} \mathrm{C}$. in fifteen minutes. A 1 per cent solution of carbolic acid was fatal to it in 5 minutes. It resists drying when in films on cover-glasses for from 7 to 15 days.

"Pathogenesis. This organism is fatal to fowls, pigeons, rabbits, guinea pigs, and mice. Other animals have not been tested. Excepting in intravenous injections, comparatively läge quantities of a pure culture trere required to produce fatal results. Fowls inoculated in the wing rein with 0.3 c.c. of a fresh bouillon culture died in from three to thirteen days; usually on the fifth or sixth day. The temperature begins to rise on the second day after inoculation. It reaches 109 to $111^{\circ} \mathrm{F}$. a fer days 
before death occurs. In cases where the fowls live from five to six days they appear perfectly well for at least three days, when the feathers begin to have a slightly ruffled appearance. Pigeons inoculated with 0.2 c.c. of a bouillon culture die in from four to five days. In rabbits the lesions resemble very closely those produced by attenuated hog cholera bacteria (B. suipestifer). Guinea pigs inoculated in the abdominal cavity with from 0.2 to 0.3 c.c. of a bouillon culture die in from five to eight days."

Differences from B. pullorum. The two causative organisms differ materially in their action on sugar media. B. pullorum produces gas in dextrose, mannite and levulose, while $B$. sanguinarium does not produce gas in any of the sugars in common use. Milk is rendered acid by $B$. pullorum and alkaline by $B$. sanguinarium with or without saponification in the latter instance. $B$. pullorum is negative to the methyl red test when grown in 1 per cent maltose bouillon, while $B$. sanguinarium gives a positive reaction.

Symptoms. These are quite similar to those of other acute septicemic diseases. Drowsiness and indifference to surroundings are marked. There is loss of appetite and general weakness. The head may be drawn in close to the body or may hang limp. The mucosæ of the head are pale. Diarrhea is present in the majority of cases. The droppings are yellowish and may be tinged with green. The comb and wattles are usually paler than normal but may be darkened with venous congestion. The blood is pale red in color and presents a marked decrease in red cells and great increase of white cells. The period of incubation is four to six days and the duration of symptoms in fatal cases from four to twelve days. A temperature elevation of three to five degrees is noted.

Morbid anatomy. There is a general anemic appearance of the serous membranes. The mucous membrane of the intestine is usually pale. At times it may show slight areas of congestion, or hemorrhagic points. The most marked changes observed on post mortem examination are in the liver, spleen, kidneys and blood. The liver is greatly enlarged and generally dotted with grayish necrotic spots. It may, at times, be congested throughout or show spots or bands of congestion. It has a tendency to become friable. Microscopic examination of sections of the organ reveals an engorgement of the blood ressels and a breaking down of the parenchymatous tissue. The hepatic cells present different stages of degeneration from cloudy swelling to complete necrosis. The necrotic areas vary in size from that of a few cells to macroseopic lesions. The spleen is sometimes 
enlarged, dark colored and pulpy. It may show necrotic points. The kidneys are somewhat enlarged, may be lighter in color and slightly injected with blood.

The raseular system shows the most constant changes. The heart appears normal or else pale with grayish spots of necrosis. The blood is lighter in color and does not clot readily. Microscopic examination reveals a great disproportion between the number of red and white cells present. The red cells decrease in numbers to a marked degree as the disease advances while the white cells become greatly increased in numbers. This increase is confined principally to the polymorphonuclear leucocytes. The following table from Moore will illustrate the proportionate relationship between the red and white cells during the course of the disease.

Table III.-Blood Changes in Fowl Fed Culture March 26.

\begin{tabular}{|c|c|c|c|c|}
\hline Date & $\begin{array}{l}\text { Temper- } \\
\text { ature }{ }^{\circ} \mathrm{F}\end{array}$ & $\begin{array}{l}\text { Number of } \\
\text { red corpus- } \\
\text { cles per } \\
\text { c. } \mathrm{mm} \text {. }\end{array}$ & $\begin{array}{l}\text { Number } \\
\text { of white } \\
\text { corpuscles } \\
\text { per } \\
\text { c. } \mathrm{mm} \text {. }\end{array}$ & Remarks. \\
\hline Mar. 26 & 106.2 & $3,535,000$ & 18,940 & Well. \\
\hline Mar. 28 & 110.0 & $2,430,000$ & 70,000 & Fowl eats very little. \\
\hline Apr. 2 & 110.6 & $1,684,210$ & 80,000 & $\begin{array}{l}\text { Blood very pale; fowl } \\
\text { weak; refuses food. }\end{array}$ \\
\hline Apr. 3 & 106.0 & $1,745,000$ & 245,000 & $\begin{array}{l}\text { Very weak; many red } \\
\text { corpuscles attacked by } \\
\text { leucocytes. } \\
\text { Found dead. }\end{array}$ \\
\hline & & & & Found dead. \\
\hline
\end{tabular}

In stained preparations of the blood numerous red corpuseles are obscrved undergoing degeneration. This is manifested br the failure of the cellular protoplasm surrounding the nucleus to stain regularly or at all, and also by vacuolization of the protoplasm of the cell.

Bacterium sanguinarium may be recovered in pure culture on artificial culture media from the blood, liver, spleen and kidners of fowls recently dead of the disease.

Differential diagnosis. Fowl typhoid is distinguished from fowl cholera by the absence of severe congestion of the mucous membrane of the intestine especially the duodenum, br the alsence of hemorrhagic spots on the heart, by the failure to find bipolar staining bacteria in the blood, and by the finding and isolation of $B$. 
sanguinarium. Also, the duration of symptoms is apt to be much shorter in the case of cholera.

Fowl typhoid is differentiated from fowl pest by the absence of severe intestinal congestion, failure to find petechiæ in the mucosa of the proventriculus and by the fact that fowl pest is caused by a filterable virus and no organisms can be detected in the blood stream, or isolated on artificial culture media.

Infectious leukemia has somewhat similar lesions and also shows a greatly changed condition of the blood. However, in this disease the loss of red cells and increase of white cells is much more marked than in fowl typhoid. Also, it is the mononuclear cells, instead of the polymorphonuclears, which show the principal increase in numbers in infectious leukemia. Unlike fowl typhoid, infectious leukemia is caused by a filterable virus and no microorganism can be grown, from the blood or organs on artificial culture media.

Fowl typhoid and acute Bacterium pullorum infection of grown fowls have symptoms and lesions which are somewhat similar. The causative organisms also closely resemble each other both morphologically and in their cultural characteristics. In B. pullorum infection, however, the ovary is congested and nearly always presents several hard irregular ova. It is the principal seat of predilection for the pullorum bacterium.

\section{PSITTACOSIS OF PARROTS}

Synonyms. Septic fever of the parrot, parrot septicemia, (English); septicemie des perruches, mycose des perroquets, (French); Psittacosis, (German).

Characterization. The disease is a contagious septicemia involving parrots and parrakeets and causing fever, weakness, stupor and diarrhea.

Transmission to man. The disease has received considerable attention because suspicion has been aroused that the infection may be transmitted to man. A number of outbreaks of infectious pneumonia in man have been observed to occur simultaneously with the bird disease.

Leichtenstern sums up the question of the transmission of the disease to man as follows:

"1. Epidemiological, clinical and especially bacteriological evidence is not disclosed to prove that the infection of house epidemics of pneumonia suspected of being psittacosis, did originate from sick parrots.

" 2 . It is proven that bacterial diseases, especially enteritis, occur fre- 
quently in parrots, particularly those freshly imported, and inflict sporadic and also excessive mortality.

"3. No one will doubt that the streptococci, staphylococci, pneumococei, coli and proteus varieties occurring in these fatal parrot diseases may under certain circumstances also become dangerous for man.

"4. The disease of man designated psittacosis is in elinical and anatomical features an atypical pneumonia coupled with typhoid symptoms and the disease has the same character in all epidemics.

"5. The same house epidemics of pneumonia occur not rarely, without the intervention of parrots and one may from this draw the conclusion that all the former psittacosis epidemics were nothing more than atypical pneumonia in which the sick parrot, accidentally present in the house, played no etiological rôle.

" 6 . The above conclusions under 5 are contradicted in some measure by the no small number of former so-called psittacosis house epidemies. Especially contradictory to these conclusions is the Paris epidemic of 1892 in which the transmission of the disease from the sick parrots to man must be considered on the basis of epidemiological facts as, at the least, apparently wholly indicated."

Etiology. An organism belonging to the hog cholera group has been isolated from cases of the disease in parrots, by Nocard and by Palamidessa. It is an actively motile, Gram negative rod which grows equally well under aerobic or anaerobic conditions. The growth in bouillon and on agar presents no characteristic features. Growth on gelatin at first consists of a shiny, transparent, iridescent streak which develops into a porcelain-white growth. No liquefaction of gelatin occurs, and milk is not coagulated. Growth on potato resembles that of $B$. coli. The organism may be isolated from the blood, bone marrow, spleen and other organs of infected birds. It has been isolated from the intestinal contents of parrakeets which were in apparent good health.

Pathogenesis. The causative organism is pathogenic for the parrot, parrakeet, pigeon, fowl, mouse, rabbit and guinea pig. All discharges of an infected bird are infective and in view of the close contact between birds there is every opportunity for transmission of infection. The disease occur's most frequently among birds during shipment from the tropics and shortly after landing. Contributory factors to its occurrence seem to be the unnsual restraint, crowding. filthy cage conditions, climatic conditions and general hardships suffered during shipment.

Symptoms. The disease is manifested by shivering, inappetence, diarrhea with frothy greenish droppings which are sometimes blocdy. The bird exhibits great thirst, drowsiness and disinelination 
to move. The wings droop, the eyes are closed, the feathers are ruffled and the head is turned on the shoulder. When attempt is made to move, great weakness is evident. The bird usually avoids the perch. Convulsions precede death by a short period. In more chronic cases there is a discharge from the nostrils and eyelids, coughing, snuffling and difficult, panting respiration. Emaciatiou is evidenced by prominent breast bone and loss of weight. Vomiting is occasionally observed.

The disease is usually fatal and hope of recovery may be entertained only in eases which survive for eight or nine days and which display some appetite. Most die in from three to five days after siçness is noted.

The incubation period in natural exposure varies from three days to several weeks.

Morbid anatomy. The organs of the abdominal cavity show intense congestion and ecchymoses are present in the peritoneum. Small grayish spots may be observed in the liver, spleen and kidneys. The spleen is usually enlarged and soft in texture. The intestines show general congestion, or catarrh and often contain ulcers on the mucosa. The muscles often present a striated and spotted appearance. The heart muscle is often dark or hemorrhagic. Ecchymoses may be present in the endocardium and pericardium. The lungs may present various conditions such as total consolidation, yellowish pneumonic patches, areas of atelectasis or of congestion. The air sacs may contain fibrinous or fluid exudate.

Treatment. The patient should be kept in a room having a temperature of from $90^{\circ}$ to $100^{\circ} \mathrm{F}$. continuously. Gray advises that aspirin or cyllin may be given in the drinking water. In cases showing improvement, the aspirin may be replaced by aromatic sulphuric acid and quinine sulphate. Food may consist of bread and milk, banana, sponge cake soaked in sherry, etc.

Prevention. The most effective prevention would consist of improvement of methods of isolation of individuals and of general conditions on shipboard. Prophylaxis on shore involves perfect segregation of birds and the application of disinfection.

DISEASE OF PIGEONS CAUSED BY A BACILLUS OF THE HOG CHOLERA GROUP

Moore observed an infection in pigeons in New Jersey caused by an organism closely related to Bacillus suipestifer. 
Symptoms. The disease is characterized by emaciation and a peculiar turning of the head from one side to the other. Occasionally there are paroxysms of aimless flying about. The disease is popularly designated "megrims."

Etiology. The organism belongs to the hog cholera group of bacteria but differs in several particulars from the speeific organism isolated from affected hogs. The organism from the pigeon is appreciably larger. In bouillon a delicate pellicle develops on the surface of the fluid, and in old cultures, a deposit is formed on the sides of the tube. A marked indol reaction occurs. The pigeon culture is less rapidly fatal for experimental animals than $B$. suipestifer.

Morbid anatomy. Autopsy of a bird that had shown the peculiar head movement for several days revealed no lesions in the thoracic or abdominal cavities. There was a friable exudate about $1 \mathrm{~mm}$. in thickness, in the subarachnoid space over the cerebellum and the posterior lobes of the cerebrum. It was grayish rellow in color and easily removed. The subjacent brain tissue was reddened but otherwise the brain was normal in appearance. The organism in question was recovered in pure culture from the brain lesion but not from the heart blood. Another pigeon found dead, showed great emaciation but few other changes beyond pale, fatty heart muscle. The same organism was recovered in pure culture from the liver and blood.

\section{AN INFECTION IN PIGEONS CAUSED BY BACILLUS PARATYPHOSUS B.}

Zingle observed an infection in pigeons apparently caused by $B$. paratyphosus $B$.

Morbid anatomy. The external appearance of the careass, beyond marked emaciation, presents nothing suspicious. The oral cavity in all cases is free from lesions. Upon skinning, the breast muscle is seen to be colored yellow in some places and permeated with bright yellow knots like oat grains. The liver frequently has a yellowish gray color, and on closer observation it is seen to be envered orer the whole surface with countless hyperemic areas, which are sharply defined from the surroundings. In more cases the liver is permeated with yellow, round, compact knots varying in size from that of a grain of wheat to that of a bean, which give the surface a roughened appearance. The spleen is generally small without particular macroseopic changes. The intestine appears somewhat hem- 
orrhagic. The most marked lesions are shown by the kidneys. These are extraordinarily large, and the borders of the several lobules have completely disappeared. The color of the kidneys is mostly gray-yellow. They appear to be sprinkled through with numerous speck-like foci and they are very fragile in consistency. The lungs appear free from lesions in many cases. Frequently, however, the lungs contain numerous opaque, gray-white foci the size of a hemp seed which are uniformly distributed. Lesions are not observed in the trachea, but the mucous membrane of the crop in isolated cases contains yellow crumbly, button-like areas of exudate.

Etiology. In the bacteriological examination of blood, musculature and organs a bacillus was found which agreed with paratyphoid B in cultural, morphological and serological characters. The culture agglutinated in a dilution of $1: 15,000$ completely and partially at $1: 25,000$ with $B$. paratyphosus $B$ serum of a titre of $1: 30,000$.

All the strains showed the same characteristics as $B$. paratyphosus $B$ in litmus milk, glucose, Endo agar, malachite green agar, gelatin, Barsiekow solution 1 and 2, neutral red agar, orzein agar as well as milk, potato and bouillon.

Pathogenicity. White mice succumbed to subcutaneous injection in two days. It was especially noticeable that the organism occurred in great numbers, and was never observed mixed with other organisms.

Pathological histology. In microscopic sections of the kidneys there are found inflammatory foci somewhat larger than a millet seed with round cell infiltration of the interstitial area. Also parenchymatous degeneration of the epithelium of the convoluted tubules in that the nuclei of the same in part are poorly stained and the protoplasm appears to be granular. The lumina of the tubules are filled with masses of desquamated epithelial tissue. When stained with Sudan they show fatty degeneration of medium degree in the epithelium of the labyrinths.

The investigation of the liver shows that the organ is permeated with numerous abscesses.

The knots in the musculature present themselves microscopically as abscesses varying in size from that of an oat grain to that of a pepper seed, with distinct lymphocyte walls which mingle with the neighboring muscle fibers. In the center of the abscesses there is much fibrin and also pus cells. 
In the lungs there are numerous abscesses analogous in structure to those in the muscle.

Relation to bird pox. Because of a suspicion that bird pox virus was present, inoculation experiments were undertaken. By rubbing of pus from liver, kidneys, lungs, ete., as well as blood and musculature, skin, muscle nodules, and crop exudate, into scarified breast skin of pigeons, there appeared after 2 to 3 days, yellowish swellings which looked exactly like pox. After a week they reached the maximum size of a pea. During this time the breast muscle fell away very markedly. These swellings began to shrink and dry up, while as a rule no fresh swellings appeared. Some of the pigeons died within 2 to 4 weeks after inoculation. By that time the poxlike swellings on the breast skin were totally dried up or had fallen off. The musculature was atrophied, flecked with yellow and in some cases contained numerous knots shaped like an oat grain with section findings like those in the original pigeons.

Other pigeons lived. After some time the pox scabs fell oft, the breast muscle filled out, so that the bird appeared clinically healthy.

These results were obtained easily through inoculation of generation after generation, also with dried out material, and the $B$. paratyphosus $B$ could be recovered each time.

The question arose as to whether these organisms were the primary cause of this pigeon disease or whether like hog cholera, the paratyphoid bacillus played a part of a leader. There was the possibility of a mixed infection with bird pox, and on this account experiments were started with contamination free filtrates of cultures and organs.

Also inoculation experiments were undertaken with cultures that had been grown for many generations in fluid and solid media.

While the filtrate experiments gave no positive result, inoculations with pigeon paratyphosus culture always gave pox-like appearances in two days.

\section{AVIAN SALMIONELLOSIS}

Lignières and Zabala deseribe an infection in fowls caused by an organism which they regard as showing all the specific characters of the hog cholera bacillus. The authors note that their organism is non-motile, while the hog cholera organism of Salmon and Smith is motile. However, they conclude that this difference is not sufficient to differentiate the organisms. Consequently they propose the name avian salmonellosis for the disease observed. 
Etiology. The organism isolated is a non-motile rod which is Gram negative. Whether a precipitation of casein occurs in milk or not, the medium becomes liquid again in 5 to 8 days. The color is grayish and the reaction is strongly alkaline. Indol is not produced and gelatin is not liquefied. Growth occurs on potato. There is nothing distinctive about growth on other media.

Pathogenicity. It is possible to inoculate healthy hens and rabbits with several drops of infected blood without killing them. Subcutaneous inoculation with culture does not always kill hens but intravenous injection of 1 to 2 c.c. of culture has a fatal result. The pigeon is easily killed by intravenous and subcutaneous inoculation. Ducks and geese are resistant to the infection. The rabbit inoculated intravenously with 1 c.c. of culture is killed, while subcutaneous inoculation merely causes tumefaction at the point of inoculation.

Symptoms. No symptoms characteristic of the infection in question are displayed.

Morbid anatomy. The comb is black, and sometimes dark ecchymoses are observed on the skin. There is a clear fluid in the pericardium. The liver and the kidneys are congested. The spleen is enlarged and much more so than in fowl cholera. The mucosa of the digestive tract is reddened in places, but hemorrhages are rarely found.

PATHOGENICITY OF B. ENTERITIDIS AND B. PARATYPHOSUS B TO BIRDS

Reinholdt has found that introduction of either $B$. enteritidis or $B$. paratyphosus $B$ into hens, pigeons, geese and ducks, sometimes causes a transitory disease and sometimes a fatal disease. This is true of a wide variety of methods of inoculation such as intravenous, intraperitoneal, subcutaneous and intramuscular injection, as well as administration by the stomach. Pigeons are most susceptible to infection, followed in order by geese and hens. Administration of the organisms per os under normal conditions of feeding is of least influence. A very large number of organisms is necessary to induce infection. The organisms are always recovered when the bird dies, but this is not always true when a bird is slaughtered while healthy. Agglutinins are detected six days after infection. 
Fowls. Outbreaks of septicemic infections in fowls caused by B. coli have been reported by Lignières, MIartel, Claussen, Zeiss and Schlegel.

The symptoms are practically indistinguishable from those of fowl cholera. The birds show somnolence, depression, diarrhea and inappetence. In the late stages, the comb may be dark colored as in cholera.

At autopsy, there is inflammation of the intestines with hemorrhages in places. There may be a small amount of serous exudate in the abdominal eavity. The spleen is softened and hypertrophied. There is pericarditis manifested either by the presence of hemorrhages, solid exudate or fluid exudate. Microscopic examination of stained smears from the blood and organs shows numerous bipolar staining rods. However, the organisms average twice as large as those of fowl cholera.

In inoculation experiments made by Lignières, hens resisted subcutaneous and intramuscular injection of 1 to 2 c.c. of culture. On the other hand the pigeon succumbed to a dose of 1 c.c. The same dose injected subcutaneously into the rabbit and guinea pig caused only a local abscess, while intrapleural or intraperitoneal injection killed.

Martel killed hens by intramuscular injection of small amounts of culture. Failure resulted from attempts to cause infection by intravenous injection or by ingestion of cultures, exerement and virulent products. The guinea pig, rabbit, white rat and white mouse were found to be susceptible to injections.

Claussen isolated a strain that was uniformly fatal to canary birds, white mice and guinea pigs. Pigeons, hens and ducks became sick only exceptionally. The infection led to the death of only 50 per cent of the inoculated birds, while the remainder recovered after three to six days. The incubation period was on an average, twelve hours long. Rabbits were very refractory to inoculation.

Claussen concludes that colon organisms occurring in the intestines of healthy hens possess the ability of becoming virulent under certain conditions and cause a cholera-like infection. These special conditions are believed to be furnished by the harmful influence exerted by shipment for long distances with resulting hunger, thirst, cold and lack of ventilation.

Zeiss reports a case in which two hens in a healthy flock sud- 
denly displayed lack of appetite with depression and died soon. Autopsy revealed extraordinary softness of the spleen and liver together with limited subcutaneous hemorrhages. Preparations from the blood revealed bipolar rods. The organism isolated from the cases showed the morphology and biologic characteristics of $B$. coli.

The organism was pathogenic for canaries. On the other hand, guinea pigs could not be infected by either subcutaneous nor intravenous injection with 2 c.c. doses of 24 hour bouillon culture.

Pigeons. Sanfelice observed an outbreak of a disease among pigeons caused by $B$. coli. Most of the birds appeared at autopsy as follows: On opening the abdominal cavity a sero-fibrinous exudate is noted on the whole surface of the intestines. The abdominal fluid is increased in amount. The spleen is swollen. There are adhesions of the intestines, the heart and the liver. The exudate appears in the form of a pseudomembrane in that when grasped with tweezers, it pulls off completely. In some pigeons besides the peritonitis described, there is inflammation of the mucosa of the oviduct, which is covered with a purulent exudate. Often eggs are found in the oviduct, which could not be laid on account of the inflammation. An organism identified as $B$. coli was observed in smears and was recovered in culture from the organs. Pigeons inoculated subcutaneously with one to one and one half c.c. of culture, developed an abscess at the point of inoculation but did not die. Larger doses injected into the abdominal cavity caused death.

Pheasants. Klein described an organism isolated from young pheasants dead of an infectious disease. The organism was motile, somewhat shorter than $B$. coli and did not coagulate milk. $\mathrm{He}$ concludes that the organism, though belonging to the same family as $B$. coli, was a different species.

\section{GROUSE DISEASE}

Under the name of grouse disease, Klein has described a pneumonia caused by a member of the B. coli group. Subsequent investigation by a committee of inquiry on grouse disease revealed the fact that the condition of the lungs described by Klein represents post-mortem changes. The supposed etiological agent is regarded by the committee as a post-mortem invader. It was noted that Klein had opportunity to study only birds that had been dead several days. The cause of mortality among grouse was watched by the committee very closely for six years and in no instance was 
there observed an epizootic like that described by Kilein. A large number of agencies are responsible for the death of grouse, and probably the most important is infestation with Trichostrongylus pergracilis as pointed out first by Cobbold.

\section{QUAIL DISEASE}

\section{Synonym. Colibacillosis tetraonidarum.}

Characterization. Quail disease is an infectious disease which according to MTorse is caused by a member of the colon group and characterized by congestion of the lungs, focal necrosis of the liver and ulceration of the intestine. It has been recognized only among birds in captivity.

Species affected. The disease has been observed in the bobwhite (Colinus virginianus), the California quail (Lophortyx californicus vallicola), the Gambel quail (Lophortyx gambeli), the mountain quail (Oreortyx pictus), the scaled quail (Callipepta squamata) and the sharp-tailed grouse (Pediocaetes phasianellus campestris).

History. The disease for many years has been observed among quail shipped for stocking areas of land and appears during transportation, or immediately after. It seems evident that the unusual conditions and hardships endured during the shipment constitute an important factor predisposing to the occurrence of the infection. Many outbreaks occur among quail which have been recently captured in Mexico. Miorse has written an article summing up the available information regarding the characteristics of the disease.

Etiology. Mforse reports finding a bacillus of the B. coli group present in diseased birds, with striking constancy. Cultures are readily isolated from the liver and intestines. It cannot always be cultivated from the heart blood of birds dead of the disease, nor always from the lungs.

Pathogenesis. The organism is not pathogenic for chickens, pigeons nor rabbits but kills mice and guinea pigs with characteristic lesions. Experiments with quail are hindered by the difficulty in obtaining birds that have not been exposed to infection.

Symptoms. The srmptoms first become manifest by dullness, inappetence and ruffling of the feathers. Death may oceur within two or three days after the appearance of symptoms. In chronic cases emaciation becomes extreme.

Morbid anatomy. Is a rule, the carcass is in good condition. 
There is slight congestion of the lungs, and of the liver. The surface of the latter organ presents a few areas of necrosis. Sometimes the liver contains no necrotic spots and is merely congested, but in some cases the necrosis involves a large area. The intestinal wall contains numerous small yellowish areas of necrosis which may be seen through the peritoneal surface. These may be exceedingly minute, or reach the size of a pinhead. In some cases the necrotic process appears in the form of ulcers, some of which may perforate the intestinal wall. In other cases the intestinal lesions may consist of mere reddened areas.

Prophylaxis. It is likely that the removal of birds from the natural wild environment, interference with natural food supply, crowding in cages and shipment are the fundamental factors predisposing birds to infection. These undesirable conditions of unnatural crowding are difficult to correct.

The cleaning and disinfection of shipping eages should be carried out very thoroughly. So far as possible, birds should be isolated in small lots during shipment. All possible precautions to prevent the contamination of feed and water should be carried out.

Treatment. The nature of the disease and subjects are such as to prevent successful treatment.

ASTHENIA IN FOWLS

(GOING IIGHT)

Characterization. The affection is characterized by extreme emaciation associated with reddening of the walls of the duodenum.

History. Dawson described the disease as occurring in the vicinity of Washington, D. C.

Etiology. The organism isolated from the duodenum of affected birds is designated Bacterium astheniev and is regarded as a variety of the colon species.

Pathogenicity. Dawson was unable to induce disease in chickens by feeding organs or cultures, or by inoculating them intraabdominally, subcutaneously and intravenously. Nevertheless he was inclined to believe that the organism in question was the cause of the disease.

Dawson considers that the presence of the organism in the part of the alimentary canal where the digestive process is most active 
interferes with nutrition. Pigeons, mice and rats proved equally refractory. A guinea pig inoculated subentaneously with duodenal contents sucemmed to the infection. Tabbits inoculated subentaneously with culture and fed the same, proved refractory. Intraabdominal inoculation of half a enbic centimeter of culture proved fatal to a rabbit.

Symptoms. Extreme emaciation is the most noticeable stmptom. The appetite is voracious. There is no evidence of diarrhea and no increase of temperature. The comb and wattles are slightly more pale than normal.

Morbid anatomy. Autopsy of affected fowls reveals extreme emaciation of the muscular system and riscera with almost complete absence of fat. There is reddening of the walls of the dundenum and the contents are mucoid in appearance.

Treatment. Dawson recommends purgation to remove the organisms in the duodemum. For this purpose he administers castor oil in a dose of two teaspoonfuls or calomel in one fourth grain doses repeated frequently. He suggests that purgation be followed by a stimulating tonic recommented by Trégnin as follows: Powdered fennel, anise, coriander seed, cinchona, each 30 grains; powdered gentian and ginger, of each 1 dram; powdered sulphate of iron, 15 grains. Mix. Add from 2 to 4 grains of this mixture for each fowl to the food twice a day.

\section{REFERENCES}

1. Claussen. Ueber Kolibakterienseptikämie bei Hühnern als Transportkrankheit. Ztschr. f. Infel.tionskr. d. Haustiere, Bd. 3, 1907, S. 69.

2. Committee of inquiry on grouse disease. The grouse in health and disease. London: Smith, Elder \& Co., 1911.

3. Curtice. Fowl typhoid. Rhode Island Agr. Exp. Sta. Bull. S7, 1902.

4. Dupuy. De la psittacose au point de vue epidemiologique. Prog. Med., 1897, p. 225 .

5. Hadley. Infections caused by bacterium pullorum. Rhode Island Agr. Exp. Sta. Bull. 172.

6. Klein. Ueber eine akute infektiöse Krankheit des schottischen Moorhuhnes (Lagonus scoticus). Centralbl. f. Baliteriol., Bd. 6, 1889, S. 36.

7. Kilein. Ein weiterer Beitrag zur Kenntniss des Bacillus der Grousedisease, Centralbl. f. Bakteriol., Bd. 6, 1589, S. 593.

8. Kilein. Ueber eine epidemische Tírankheit der Hühner, verursacht durch einen Bacillus-Bacillus Gallinarum. Centralbl. $f$. Baliteriol., Bd. 5, 1889, S. 689 .

9. Leichtenstern. Ueber infektiöse Lungenentzundungen und den 
heutigen Stand der Psittacosis-Frage.-- Werden durch spezifish erkrankte Papageien bösartige Lungenentzundungen beim Menschen hervorgerufen? Centralbl. allgem. Gesundheitspf., Bd. 18, 1899, S. 241. Abs. in Centralbl. f. Bakteriol. (Etc.), 1. Abt., Orig., Bd. 26, 1899, S. 651.

10. Lignières. Septicémie a coli-bacille chez la poule Compt. rend. Soc. de biol., T. 46, 1894, p. 135.

11. Lignières and Zabala. Sur une nouvelle maladie des poules. Bull. Soc. Cent. de Méd. Vét., T. 59, 1905, p. 453.

12. Martel. Maladie a coli-bacille de la poule et de la dinde. Compt. rend. Soc. de boil., T. 49, 1897, p. 500.

13. Moore. On a pathogenic bacillus of the hog-cholera group associated with a fatal disease in pigeons. U. S. Dep. Agr. Bur. Animal Indust. Bull. 8, 1895.

14. Moore. A study of a bacillus obtained from three outbreaks of fowl cholera. U. S. Dep. Agr. Bureau Animal Indust. Bull. 8, 1895.

15. Moore. Infectious leukemia in fowls - A bacterial disease frequently mistaken for fowl cholera. U. S. Dep. Agr. Bureau Animal Indust. 12th and 13th Ann. Rep., 1895-96.

16. Morse. Quail disease in the United States. U. S. Dep. Agr. Bureau Animal Indust. Circ. 109, 1907.

17. Palamidessi. Di una infectione nell' womo transmesso probabilmenti dai pappagalli. Policlinico, 1895. Abs. in Centralbl. f. Bakteriol. (Etc.), 1. Abt., Orig., Bd. 20, 1896, S. 316.

18. Pfeiler und Rehse. Bacillus typhi gallinarum alcalifaciens und die durch ihn verursachte Hïhnerseuche. Mitteil. a. d. Kaiser Withelm Institut f. Landwirthschaft in Bromberg, Bd. 5, 1913, S. 306; Abs. in Cen. tralbl. f. Balteriol. (Etc.), 1 Abt. Ref., Bd. 58, 1913, S. 575.

19. Pfeiler and Roepke. Zweite Mitteilung über das Auftreten des Hühnertyphus und die Eigenschaften seines Erregers. Centralbl. f. Bakteriol. (Etc.), 1 Abt. Orig., Bd. 79, 1917, S. 125.

20. Reinholdt. Infektionsversuche mit den "Fleischvergiftern" (Bacillus enteritidis Gärtner und Bacillus paratyphosus B) beim Geflugel. Centralbl. f. Bakteriol. (Etc.), 1. Abt. Orig., Bd. 62, 1912, S. 312.

21. Rettger and Koser. A comparative study of Bacterium pullorum (Rettger) and Bacterium sanguinarium (Moore). J. Med. Research, Vol. 35,1917, p. 443.

22. Sanfelice. Eine Seuche dei Tauben durch Bacterium coli verursacht. Ztschr. f. Hyg. u. Infektionskr., Bd. 20, 1895, S. 23.

23. Smith and Ten Broeck. Agglutination affinities of a pathogenic bacillus from fowls (fowl typhoid). (Bacterium sanguinarium, Moore.) J. Med. Research, Vol. 31, 1915, n. 503.

24. Smith and Ten Broeck. The pathogenic action of the fowl typhoid bacillus with special reference to certain toxins. J. Med. Research, Vol. 31,1915 , p. 523.

25. Smith and Ten Broeck. A note on the relation between B. pullorum (Rettcer) and the fowl typhoid bacillus (Moore). J. Med. Research, Vol. 31, 1915, p. 547.

26. Taylor. A report upon an outbreak of fowl typhoid. J. Am. Vet. M. Ass., n. s. Vol. 2, 1916, p. 35. 
27. Zeiss. Koliseptikämie bei Hühnern. Archiv f. Hyg., Bd. 82, 1914, S. 27.

28. Zingle. Untersuchungen iiber eine Taubenseuche mit Paratyphus-BBazillenbefund. Zeitschr. Infehtionskr. d. Haustiere, Bd. 15, 1914, S. 269. 


\section{CHAPTER VI}

\section{BACILLARY WHITE DIARRHEA}

Characterization. Bacillary white diarrhea is an acute, highly fatal septicemic disease of young chicks caused by Bacterium putlorum. The infection also exists in adult hens in a chronic, rarely fatal form, usually confined to the ovary. The disease as it occurs in hens bears no specific name and is merely referred to as $B$. pullorum infection.

Etiology. Rettger and Harvey describe the characteristics of the organism substantially as follows:

"Morphology, staining properties, etc. The organism is a long, slender bacillus (.3-.5 micron $\mathrm{x} 1-2.5$ microns) with slightly rounded ends. It usually occurs single, chains of more than two bacilli being rarely found. It is non-motile, non-liquefying, non-chromogenic, aërobic and facultatively anaërobic. In its microscopic appearances it resembles the bacillus of typhoid fever. It is stained readily by the ordinary basic anilin dyes. It does not stain by the Gram method; neither does it retain its color when treated with dilute acetic and mineral acids. The organism does not produce spores, or at least they have never been observed.

"The maximum temperature tolerated is 56 to $57^{\circ} \mathrm{C}$. (moist) for an exposure of fifteen minutes. The optimum temperature is 35 to $37^{\circ} \mathrm{C}$.

"Cultural characters: Agar plates. Small white colonies make their apnearance within twenty-four hours. They increase in size slowly and seldom attain more than one millimeter in diameter, even after three or four days' incubation. Under the microscope they appear yellow and vary in form from oval and spindle-shaped to round. The surface is usually marked with a rosette figure or what seems to be a lobed nucleus. Occasionally two or even three of these markings may be seen.

"Slant agar. Growth is quite visible in twenty-four hours, and resembles that of the typhoid bacillus. It spreads little and remains delicate, even after prolonged incubation. On glycerine agar the growth is practically the same.

"Gelatin plates. Small white colonies may be seen in forty-eight hours. They remain small for several days, and only under exceptional conditions do they develop into characteristic surface colonies which to a certain extent resemble the grape-leaf colony of $B$. typhosus.

"Gelatin stab. A delicate growth occurs in forty-eight hours along the whole line of inoculation. It is of distinetly granular appearance and spreads very little on the surface. The gelatin is not liquefied.

"On potato development is very slow. A narrow, almost invisible 68 
streak is produced along the line of inoculation. In litmus milk little or no apparent change occurs within the first forty-eight hours, after which the milk becomes slightly acidified without any signs of coagulation of the casein.

"Gas production in sugar bouillon. Negative results were obtained with maltose, lactose, saccharose, inulin, and dextrin bouillon. Dextrose and mannite were attacked, however, with both acid and gas production. In the dextrose fermentation tubes about twenty per cent of the closed arm was filled with gas, and the mannite tubes averaged about the same. The gas consisted of $\mathrm{Co}_{2}$ and $\mathrm{H}$ in the ratio of $1: 3$.

"Indol and nitrite production. Neither indol nor nitrite could be detected in Dunham's peptone solution at the end of one week's growth in the incubator."

Toxin production. B. pullorum has been shown by Smith and Ten Broeck to produce a toxin fatal to rabbits. Filtrates of bouillon cultures when injected intravenously may cause death within two hour's, or marked dyspnea followed by death over night, or cause loss of weight followed by recovery. The lesions induced, include congestion of varions organs such as liver, spleen, kidneys, adrenals, lungs and mesenteric lymph glands together with hemorrhage into the gastric mucosa.

Source of infection. Infection may oceur in several ways. The most common source is through infected eggs. It has been demonstrated that affected chicks which survive the disease frequently retain the causative organisms in the system and later, in the event that the ovary becomes infected, pass them off in the eggs. Many infected eggs fail to hatch, the embryos dying in the shell at an early or late stage of development. Should one or more chicks be hatched, harboring the disease, the others of the lot are exposed early and nearly all become affected. The chicks are especially susceptible during the first 48 hours of life, but are practically insusceptible after the third day. Another source of infection is through brooders and incubators that have previously held infected chicks. Day-old chicks frequently earry the disease to other points and contaminate brooders or infect other chicks with which they come in contact. However, the largest pereentage of outbreaks is traced directly to the presence of infected hens in the flock from which the eggs for hatching were derived. There is a well defined cvcle through which the bacterium passes; namely, from the infected ovary of the hen through the egg to the chick and hack to the ovary of the pullet which has survived the disease as a chick.

Pathogenicity. Susceptibility to infection bear's a remarkable 


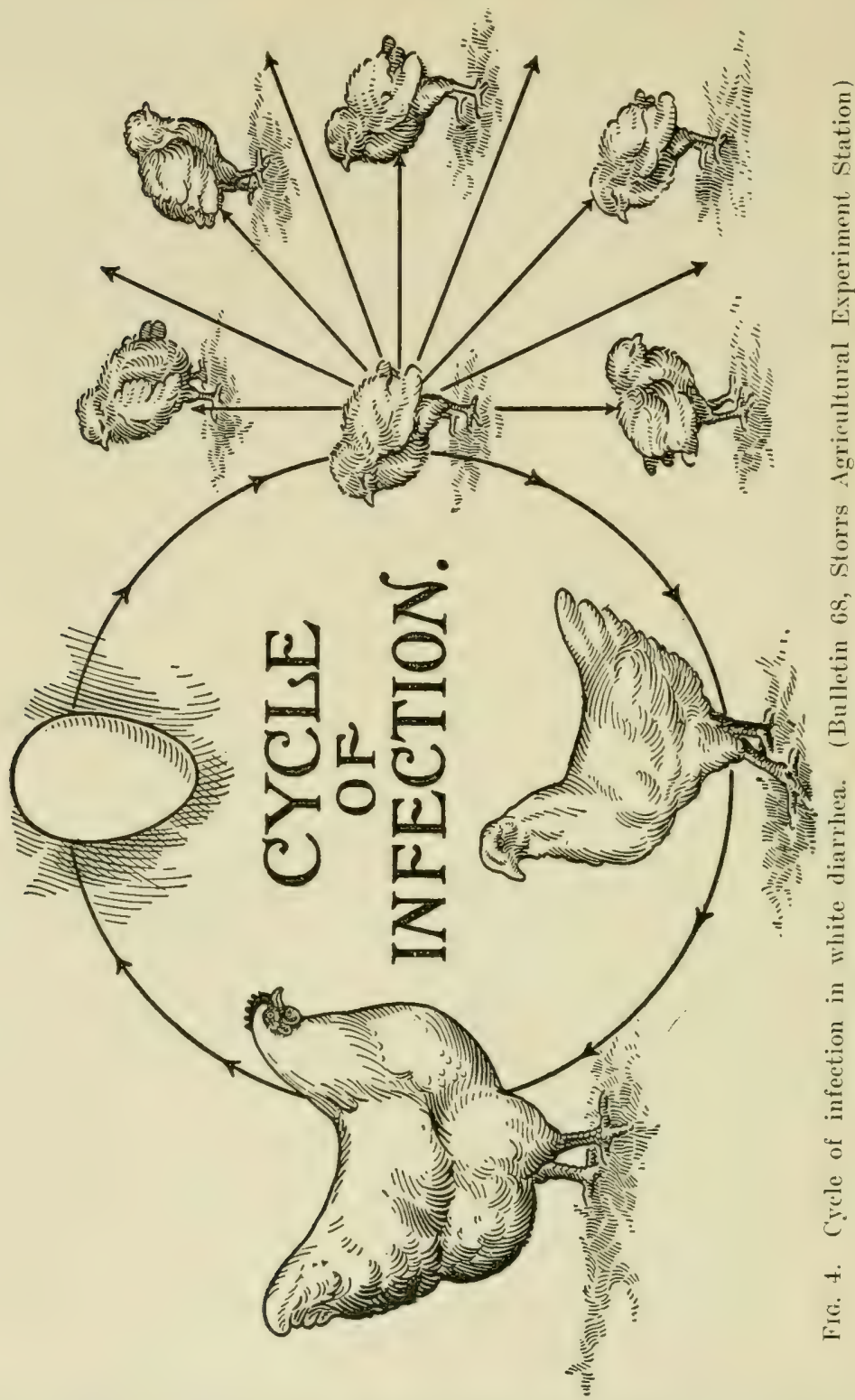

relation to age. During the first forty-eight hours the baby chick is most susceptible to Bacterium pullorum and at this period of life, has practically no resistant forces to combat the infection. In the succeeding twenty-four hours, more resistance is shown by those not 
previously affected. At the end of four days, the unattacked chicks have generally acquired a resistance to acute infection. The resistance at this age, while very apparent in broods of roung ehicks, is not absolute, since fatal outbreaks in old fowls are recorded in the literature on the disease.

Half grown guinea pigs are killed in from 24 to 48 hours when injected subcutaneously. The chief lesion is a large edematous area beneath the skin of the abdomen. Rabbits are killed in three to seven days by subcutaneous injection of one to two c.c. of 48 hour bouillon culture. In the case of all experimental inoculations, the organism may be recovered in pure culture from all the internal oreans.

Mortality. Bacillary white diarrhea is, without doubt, the most fatal disease of young chickens. Among those broods affected, the death rate varies between 40 and 90 per cent. The high pereentage is more often registered than the low one.

Symptoms. The external symptoms of bacillary white diarrhea are similar to those of coccidial and aspergillar white diarrhea. The presence of soft, whitish droppings and similar symptoms in each case caused these three diseases to be grouped under the common term "white diarrhea." The position of each as an important specific disease requires that they should be treated separately, especially since ther are not confined to young chicks but affect grown fowls as well.

The affected bird is drowsy, and dejected in appearance. The feathers are ruffled, the wings droop and the chick sways back and forth in a standing position. The appetite is diminished. The irritation of the droppings when being voided often causes the chick pain as evidenced by a peculiar cry at the time. The droppings may adhere to the down about the vent, blocking the opening. This gives rise to the expression "pasting up behind." In acute cases the chick soon falls to the floor and death occurs after a short period of coma. In more chronic cases the chick remains standing in a dull inattentive position a good portion of the time while not under the hover or hen. It acquires the appearance of having a short back and a large abdomen. Often one or both feet swell. The chick stands on one leg with the toes of the elevated foot just touching the floor. Lameness is shown in attempts to walk.

The disease may show itself within two or three days after the chicks are hatched and spread rapidly through the brool. The period of incubation is four to ten days. Deaths begin to occur 
soon after hatching and continue to the third or fourth week. Chicks which survive, remain stunted and unthrifty for some time.

Morbid anatomy. The lesions observed on autopsy are not clearly distinctive for this disease which is purely septicemic in nature. The early, rapid development of the disease, in a host unprepared to combat it, prevents the formation of many distinctive lesions. The failure of the yolk to be absorbed is the principal abnormal condition that is apparent. However, this condition is observed in very young chicks which have died through other causes, such as weak constitution, faulty incubation, and brooder defects. The liver usually shows an ochre color, with bands or streaks of congestion. This appearance is not constant as in some cases an apparently normal mahogany or chocolate colored liver is observed. In some instances the liver has the normal appearance, and the yolk sac has been absorbed. This is especially common in less acute cases. The lungs may show minute necrotic areas and, as a rule, congestion.

Diagnosis. Diagnostic features of the disease are its early appearance, the high mortality within the first month, the occurrence of deaths within a few days after hatching, and the absence of internal lesions other than unabsorbed yolk or striations on the surface of the liver. Confirmatory positive evidence is obtained by examination of stained smears of the blood, liver or yolk with discovery of the causative organism. Further evidence is yielded by feeding or inoculation experiments with young chicks.

Artificial cultural methods are more precise. The causative organism may be readily isolated in a pure culture from the heart blood, liver, spleen, kidneys or unabsorbed yolk. It may be differentiated from other organisms of a similar type by its cultural and biochemic characteristics, and by its agglutinating properties against a homologous serum.

Differential diagnosis. Bacillary white diarrhea is differentiated from the other diseases commonly classed as white diarrhea by its early occurrence, the exceptional mortality, and the absence of the characteristic lesions of coccidiosis, or of aspergillosis. Coccidiosis is slower in its development and usually affects chicks at a later pericd. It is manifested by hemorrhagic areas in the ceca, dilatation of these organs, and the presence of necrotic casts in their lumina. In the event that this disease is present, the coccidial cysts may be readily demonstrated on microscopic examination of the cecal contents. 
Aspergillosis is comparatively rare in roung chicks. Its characteristic growth may be found in the air passages, and the mreelia, or spores of the fungus can be observed br microsenpic study of the moldy growth.

Treatment. Attempts to cure bacillary white diarrhea are lintile. Some recover, owing to natural resistance, or other reasons. The so-called treatments recommended by some advisers are not to be relied upon. Once the disease has become established in the system, the causative microorganisms are distributed through the various organs by way of the blood stream and a septicemia has developerl which is beyond the reach of any antiseptic treatment which has yet been diseorered. By careful nursing, certain individuals might be assisted in surviving; however, this is inadvisable because of the fact that the monetary value of the baby chick does not justify the expenditure of the time devoted to its treatment. Furthermore, the fact that those which recover are likely to serve as reservoirs for the causative organisms and constitute the sources of future outbreaks would speak against the policy of treatment.

Where the disease is known to exist in a flock of breeding fowls and is anticipated in the hatch of baby chicks, precautions may be taken to lessen its attack by advanced treatment of the chicks. (Permanganate of potash may be placed in the drinking water in sufficient quantity to give the latter a deep red color. $\lambda$ This corresponds to dilution of approximately 1 to 10,000 . In such dilution, the antiseptic is non-irritating to the sensitive mucous membranes of the chick and may serve to inhibit the growth of the pullorum bacterium in contaminated drinking water, and in the digestive tract of the chicks. Buttermilk may be used as a food, as it combats the development of the bacterium in the intestine, due to the lactic acid which it carries.

Prevention. The first principle of prevention is the eradication of carriers in the breeding stock, or the use of eggs for hatching which come from flocks known to be free of pullorum infection. Certain tests which are later discussed may be depended upon to detect the presence of the disease in a large percentage, if not all. of the infected hens of a flock. These birds may be discarded for breeding purposes or disposed of as the owner may desire. In this connection, it has come to our attention indirectly that in rare instances normal hatches have suceeded ones in which high mortality has occurred, the eggs in each case being derived from thocks in which a number of infected hens have been detected but retained as breeder's. 
The significance of this is not apparent, but opens an interesting field for study, if the observations are accurate.

If it is desired to breed from an infected flock for the purpose of perpetuating the particular strain of fowl, or for other reasons, precaution may be taken to minimize the losses by the following procedure. Incubator trays, or shelves, of the pedigree type which are partitioned off into compartments capable of inclosing a few chicks, say from six to ten as the owner may decide, are to be used. The partitions are to be sufficiently high to prevent the chicks in adjacent compartments from coming in contact. This also means that the partitions should be of solid structure to prevent picking back and forth. It is only necessary to use such trays a day or two before the hatch is expected to come off, and for three days after it is completed. The chicks are to be retained in their respective compartments and need not be fed for 48 or even 72 hours, since they are provided with food material during this period through the yolk which is drawn into the abdominal cavity previous to hatching. Should symptoms of disease develop, the chicks in the particular compartment are to be removed and disposed of. The attendant should then disinfect his hands before watering or caring for the others. As the disease is contracted principally during the first 48 hours of life, and only to a slight extent during the succeeding 24 , this method of procedure will prevent a general spread to the hatch. The temperature and air requirements may be regulated to suit the needs of the chicks. IIumidity, due to close confinement and lack of air circulation, is to be guarded against. The trays may be removed to the brooders, or similar structures may be placed in the latter and the chicks transferred to them, with care to avoid mixing the segregated lots. After three days, the danger of infection is reduced to a minimum, and all of the hatch may be allowed to run together.

\section{BACTERIUML PULLORUM INFECTION OF GROWN FOWLS}

Occurrence. Infection of hens by the organism in question is not marked by external changes nor is it regarded as a common cause of death. Jones reports one outbreak due to B. pullorum infection which assumed an acute character with marked srmptoms and considerable mortality. In this case the fowls were infected through the feeding of eggs which had failed to hatch and which came from an infected flock. About 50 birds died during the outbreak. The 
present writer's have eneountered at autopsy a naturally infeeted fowl which had developed fatal $B$. pullorum septicemia from primary ovarian infection.

Symptoms. In the chronic form, no symptoms are apparent to the observer. The fowls continue to lay, although from the number of retained and undeveloped ova, it is evident that laying is retarded. In the acute cases reported by Jones, within a period rarying from two to six weeks after exposure by feeding, the affected birds showed a loss of appetite and energy. They appeared droopy and somnolent, with ruffled feathers and retracted heads. The combs and wattles were pale and more or less diarrhea was present. In the fatal cases, death ensued in from one to several days after the onset of srmptoms.

Morbid anatomy. In the acute cases autopsied ly Jones the most constant lesions were the presence of small necrotic foci in the liver, spleen, pancreas and heart. The capsule of the liver, the pericardium and the heart were in many instances covered by a fibrinous exudate.

In the majority of chronic cases, autopsy will show the lesions to be confined to the ovary. This organ usually presents an appearance quite characteristic. The diseased ova which have partially or wholly developed are hard, shrunken and angular. The natural yolk color has changed to a dark brown or greenish color. On cut section the ova are of a tough cheesy consistency, with a yellowish red, or greenish tinge. The condition of the ova closely resembles "gangrene of the ovary" of earlier writers. The morbid ova may also appear as cysts, with darkened fluid contents. Occasionally only small eysts are present in the ovary and it is through cultural methods alone that the presence of pullorum infection may be detected. Small foci consisting of sac-like structures with rellowish colored solid or fluid contents may occur in almost any portion of the abdominal or pleural cavities. Involvement of the pericardial sac is not uneommon.

The causative bacterium may be readily isolated from the angular ova or cysts and grown on artificial culture media. In some cases where ovarian lesions are not pronounced, it is necessary to remove the ovary and disintegrate aseptically. The mass is placed in a flask of bonillon, from which cultures may be plated on agar and the organism isolated in pure culture.

Diagnosis. Several methods of diagnosing the presence of $B$. pullorum in fowls are available and are more or less satisfactory. The presence of bacillary white diarrhea in a brood of ehicks which 


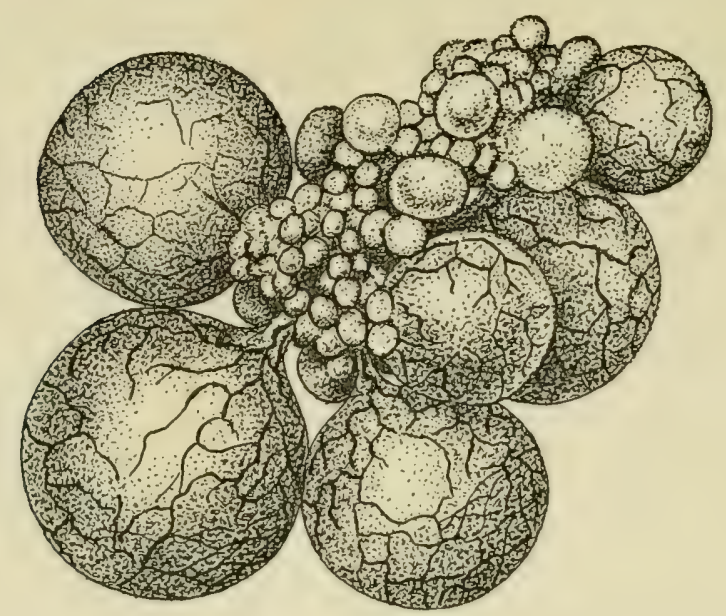

FIG. 5. Normal ovary. (Redrawn from Rettger, Kirkpatrick and Jones)

has been hatched from eggs from a particular flock of hens is proof enongh of infection in the flock, providing that there is a reasonable certainty that contamination has not been introduced by outside sources or from infected incubators or brooders. Detection of the characteristic pullorum-infected ovaries, on post mortem examination, is definite proof that the disease is prevalent in the flock. Bac-

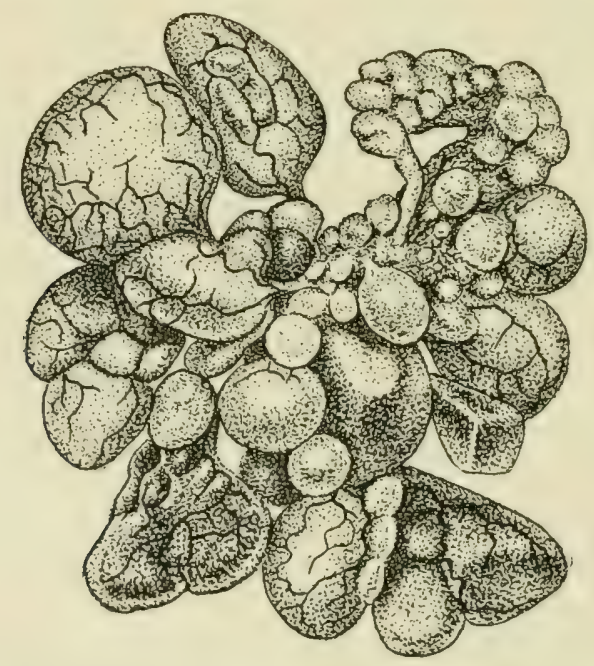

Fig. 6. Ovary infected with $B$. pullorum. (Redrawn from Rettger, Kirkpatrick and Jones) 
teriological examination of eggs from a suspected fluck also leads to a diagnosis. However, this method is impractical because of the fact that an infected hen only oceasionally drops an infected egg and considerable laboratory work is necessary to establish the presence of the disease. For the detection of individual carriers among hens held for breelers, two other methods which give positive proof of the presence of infection in the flock and pick out the infected indivichals with a high degree of accuracy have been developed. These are the agglutination test applied first by Jones, and the intradermal test developed by the authors.

Agglutination test. This test is based on the fact that the blood serum of an animal which has experienced the attack of a given species of bacteria will in some instances cause the organisms to agglutinate or form into clumps when a suspension of the bacteria is mixed with the serum of the animal in proper dilution.

The wing vein is severed at the point where it passes over the elbow joint and 3 to 5 c.c. of blood collected in a sterile test-tube or vial. The flow of blood is then stopped by momentary pressure orer the eut, or by the application of a small pledget of cotton. Usually no effort is necessary to stop the flow of blood, as chicken blood clots readily. Blood may be drawn more rapidly by severing the prominent vein visible on the ventral surface of the wing near the shoulder. The collected sample is set aside and allowed to clot. It should not be agitated while the clot is forming, as this tends to prevent the collection of a clear serum. After the clot has formed, the sample may be placed in a refrigerator or other cool place. In a few hours the serum will have been pressed out by the contraction of the fibrin and can be drawn off as a clear fluid, free of blood corpuscles or hemoglobin. If the test is not to be made immediately, the serum may be preserved by the addition of carbolic acid in sufficient quantity to form a .5 per cent solution. For this purpose, it is convenient to use a 5 per cent solution of carbolic acid as a standard, .1 c.c. being added to .9 c.c. of serum, or 1 drop to 9 drops. For the test a standardized suspension of $B$. pullorum is prepared. This mar be heated at $60^{\circ} \mathrm{C}$. for one hour, to kill the organisms, or used in the live state. The latter has been the practice of the authors. However, there appears to be no marked advantage in the use of live antigen, and killing by heat or .5 per cent carlonlization has the advantage that the organisms are dead and henee absolutely harmless.

In the test, the bacterial suspension is placed in the test tubes 
in the quantity of 1 c.c. to each tube. A series of tubes is employed for each serum, depending on the number of serum dilutions to be made. In practice, a serum which causes agglutination in a dilution of .01 or even .02 is sufficient to establish a positive diagnosis. Using 1 c.c. as the standard amount of bacterial suspension, sera which have been diluted by the addition of normal salt solution to the extent of $.04, .02$, and .01 per cent of the original, are distributed in their respective tubes in quantities of 1 c.c. each. The tubes are labeled to show the identity of the various sera and the dilutions. In practice, it is found convenient to dilute the serum to 10 per cent of its original volume and add $.4, .2$, and .1 of a c.c. to the 1 c.c. of bacterial suspension. The tubes are shaken to insure a thorough mixture of the contents and may be set aside at a temperature of $37^{\circ} \mathrm{C}$. for from twenty-four to forty-eight hours, when readings may be made. Instead, when rapid diagnosis is desired, they may be placed in an incubator at $37^{\circ} \mathrm{C}$. for $1 / 2$ hour and then centrifuged at the rate of 1500 to 1800 revolutions to the minute for several minutes. Either course gives similar results. In positive cases the organisms in the fluid collect in floceulent masses which have a tendency to form a diffuse coating over the bottom of the tube, while in the negative cases the organisms are deposited in a small circular mass in the center of the bottom of the tube. This is particularly apparent where centrifugalization has been employed. On shaking the tubes the clearly positive ones will show a clear fluid containing flocculent masses while the negative ones are uniformly clouded.

The agglutinating serum dilution which will establish a diagnosis has not been definitely fixed. It is a well known fact that normal blood serum is capable of agglutinating organisms to a greater or less degree. In $B$. pullorum infection tests, a serum which in the amount of .02 c.c. or less causes agglutination, undoubtedly indicates a positive case.

Intradermal test. This test depends for its results upon the principle underlying allergic reactions. It is very similar in character to the intradermal tuberculin test, especially as used to detect tuberculosis in fowls.

$A$ culture of several strains of $B$. pullorum from widely separated sources is grown in bouillon at a temperature of $37.5^{\circ} \mathrm{C}$. for a period of thirty days to three months. Whether these time limits are necessary has not been determined. The product is then sterilized at a temperature of $60^{\circ} \mathrm{C}$. for a period of one hour and carbolic 
acid is added in sufficient quantity to form a .5 per cent solution for preserving purposes. In the earlier experiments of the authors, the product was passed through a Berkefeld filter and enneentrated to one-tenth of its original volume. Later it was found that the original sterilized product concentrated to $1 / 5$ its volume gave as satisfactory results.

The material is injected into either wattle of the fowl in the amount of approximately .2 c.c. The injection is made with a hypodermic syringe graduated in tenths of a c.c., and a needle of fine caliber is used to avoid causing traumatic swellings. The injection is made at a point near the lower border of the wattle. Where the flock is apparently free of infectious disease, no disinfectants are employed at the

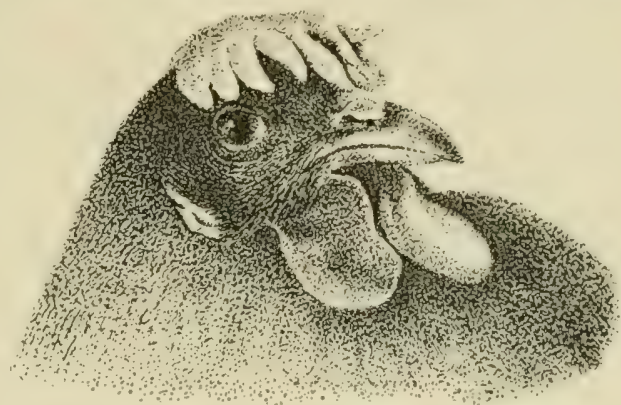

FIG. 7. Edematous swelling of wattle indicating positive reaction to the intradermal test for B. pullorum infection. (Original)

point of injection since the natural resistance of birds to ordinary wound infection is sufficient to guard against infection. In the absence of a graduated syringe, the amount to be injected may be gauged by injecting enough to cause a perceptible swelling at the point of inoculation. During the first three hours after injection the wattle shows an edematons condition which rapidly disappears in non-reacting birds. Readings are taken at twenty-four to thirty hours after injection. The wattle may be swollen to several times its natural thickness and may be readily observed at a distance, or the swelling may be so slight that palpation, or comparison with the uninjected wattle will be necessary. Any swelling other than that due to traumatism as a result of faulty technique, should be considered significant.

\section{REFERENCES}

1. Bushnell and Mraurer. The use of milk cultures of B. bulgarieus in the prevention and treatment of bacillary white diarrhea of young chicks. Am. Vet. Rev., Vol. 44, 1913, p. 195.

2. Gage and Martin. Notes on the histo-pathology of the intestines in young chicks infected with bacterium pullorum. J. Ifed. Research, Vol. 34, 1916, p. 149.

3. Gage and Hyland. On the diagnosis of infection with bacterium 
pullorum in the domestic fowl. Mass. Agr. Exp. Sta. Bull. 148, 1914.

4. Gage and Paige. Bacillary white diarrhea (Bacterium pullorum infection) in young chicks in Massachusetts. Mass. Agr. Exp. Sta. Bull. 163. 1915 .

5. Jones. The value of the microscopic agglutination test in detecting fowls that are harboring Bact. pullorum. J. Med. Research, Vol. 27, 1913, p. 481.

6. Jones. Further studies on bacillary white diarrhea in young chickens. Rept. N. Y. State Vet. Col. 1910-1911, p. 69.

7. Jones. An outbreak of an acute disease in adult fowls, due to Bact. pullorum. J. Med. Research, Vol. 27, 1913, p. 471.

8. Rettger. Septicemia among young chickens. N. York M. J., Vol. 71, 1900, p. 802.

9. Rettger. Septicemia in young chickens. N. York M. J., Vol. 73, 1901, p. 267.

10. Rettger. Further studies on fatal septicemia in young chickens or "white diarrhea." J. Med. Research, Vol. 21, 1909, p. 115.

11. Rettger. Ovarian infection in the domestic fowl and direct transmission of the disease to the offspring. J. Exper. M., Vol. 19, 1914, p. 552.

12. Rettger and Harvey. Fatal senticemia in young chickens, or "white diarrhea." J. Med. Research, Vol. 18, 1908, p. 277.

13. Rettger, Hull and Sturges. Feeding experiments with Bacterium pullorum. The toxicity of infected eggs. J. Exper. M., Vol. 23, 1916, p. 475 .

14. Rettger, Kirkpatrick and Card. Chickens: Milk feeding and its influence on growth and mortality. Comparative study of the value of sweet and sour milk. Storrs Agr. Exp. Sta. Bull. 80, 1915.

15. Rettger, Kirkpatrick and Stoneburn. Bacillary white diarrhea of young chicks. Third report. Storrs Aar. Exp. Sta. Bull. 74, 1912.

16. Rettger, Kirkpatrick and Jones. Bacillary white diarrhea of young chicks. Fourth report. Storrs Agr. Exp. Sta. Bull. 77, 1914.

17. Rettger, Kirkpatrick and Jones. Bacillary white diarrhea of young chicks. Storrs Agr. Exp. Sta. Bull. 85, 1915.

18. Rettger, Kirkpatrick and Jones. Bacillary white diarrhea of young chicks - VI. Storrs Agr. Exp. Sta. Bull. 88, 1916.

19. Rettger and Stoneburn. Bacillary white diarrhea of young chicks. Storrs Agr. Exp. Sta. Bull. 60.

20. Scherago and Benson. Experiments on the intradermal test for Bacterium pullorum. Cornell Vet., Vol. 9, 1919, p. 111.

21. Ward and Gallagher. An intradermal test for Bacterium pullorum infection in fowls. U.S. Dep. Agr. Bull. 517. 


\section{CHAPTER VII}

\section{FOIVL PLAGUE}

Synonyms. Bird plague, fowl pest, bird pest, infectious peritonitis of birds, exudative typhus of birds, Brunswick bird plague; Peste aviarire, Peste des oiseanx (French); Geflïgelpest, Huhnersenche, Huhnerpest, Vogelpest, Geflugelseuche, Kyanolophiea gallinarum, Putensenche (German); Peste aviaria, Epizoozia tifoide dell pollame, Tifo essudativo dei gallinacei (Italian).

Characterization. Fowl plagne is an acute infectious disease of birds, caused by an ultra microscopic virus. It runs a rapid course and causes high mortality.

Geographical distribution. The disease has been reported in Italy, Germany, France and Belgium.

Etiology. The virus is ultra microscopic, passes through poreelain filters, and is believed to be a protozoan. The presence of the virus has been demonstrated in the blood, the nervous system, nasal and oral discharges, serous exudates and feces.

The virus is believed to be largely localized in the red blood corpuscles. The virulence of the red corpuscles is such that death has been produced by a dose of $.000,000,000,163,84$ c.c.

Dessication is not rapidly fatal to the virus for it has been observed to remain active for several weeks. It has been rendered inactive by exposure to direct sunlight or to a temperature of $65^{\circ}$ to $70^{\circ} \mathrm{C}$. for a few minutes. The activity of the virus is destroyed rapidly by whitewash and the common disinfectants.

Certain granules have been observed in the brain of geese, hens and pigeons dead of the disease. Ottolenghi believes that they are derived from the nuclei of the cells. Certain peculiarities suggest to him the supposition that they contain the virus of fowl plague. Other granules in the brains of geese and hens are differentiated from the former by the fact that the eytoplasm of the cell is involved in their formation.

Pathogenicity. The disease has been observed in fowls, turkers, peafowls, guinea-fowls, geese, pheasants, pigeons, dueks, and a large number of wild birds. Wild ducks when placed under conditions of semi-domestication contract the infection. 
The virus is believed to be transmitted naturally by nasal and oral secretions, by the droppings of diseased birds or by eating carcasses of dead birds. Wounds of the skin when contaminated with virus readily result in infection. Mites (Dermanyssus avium) have been tested as to their ability to transmit the disease, with negative results. Likewise ticks have given negative results.

The disease is most frequent in early spring, declines in prevalence in the summer and does not occur during the winter. In artificial transmission, fowls suceumb to subeutaneous or intramuscular injection or to application of the virus to the scarified skin. They likewise become infected by ingestion.

After the subcutaneous or intramuscular injection of moderate amounts of heart blood and pieces of spleen or kidney the size of a. pea the inoculated hens die as a rule within 36 to 48 hours, often in 3 to 4 days, and quite rarely only after 5 to 7 days. Feeding of larger amounts of pieces of organs causes death after about the same length of time or a few hours longer than in the case of subcutaneous injection. Filtrates of nasal mucus, contents of the small intestine, bile, brain, blood and internal organs are infective for hens. Many authors have failed to transmit the clisease to old and young ducks or to young pigeons. Young geese are susceptible to subcutaneous and intramuscular inoculation or by the mouth, and die as a rule after about seven days. On the other hand, old geese are very slightly susceptible to ordinary methods of inoculation. Subdural inoculation of these has succeeded, and caused the nervous type of the disease.

The disease exhibits peculiarities in showing a preference at different times for different species of birds all in contact with one another, such as fowls, pigeons, geese and ducks. Mammals are immune to infection.

In young geese and in pigeons the disease assumes the nervous type, which runs a much less rapid course. In these the virus at first localized in the blood corpuscles seems to disappear therefrom, and becomes established in the central nervous system. However, the virus may reappear in the blood shortly before death.

Cominotti has observed the nervous type of the disease in wild ducks in confinement under conditions resembling those under which domesticated birds are kept. The virus was found always in the central nervous system and was never demonstrated in the blood or the internal organs even when large quantities of inoculating material were used. Transmission of the infection from wild ducks to 
fowls was possible by subcutaneous injection of portions of eerehul substance. It was not possible to infect domesticated ducks with wild duck virus in doses howerer large, and administered by intrarenous inoculation, ingestion, intracerebral innculation or instillation into the conjunctivæ.

Symptoms. The incubation period is usually 3 to 5 days, but exceptionally 2 days or even 7 days. It is much shorter when birds are inoculated. The symptoms greatly resemble those of fowl cholera. Death may occur while the bird is on the roost or on the nest without its having previously exhibited symptoms.

There is an acute type of the disease in which the bird lives at the most three or four days. In a subacute form the birds survive 7 or 8 days. Recovery practically never occurs.

In the acute type, the first noticeable symptom is inappetence. Then the bird declines into a condition of weakness and somnolence, hides in corners, becomes indifferent to surroundings, with half closed eyes, drooping comb, and ruffled feathers. The temperature rises to $110^{\circ}$ or $112^{\circ} \mathrm{F}$., but may drop to subnormal before death. The comb commonly takes on a violet color which proceeds from the borders, or spots form on the sides, but it soon appears black. In some cases the comb is covered with white scales. The bird generally dies quietly in a somnolent condition, which is only oceasionally interrupted by spasms.

In the subacute form which occurs quite frequently in cases of natural infection, the bird ceases to eat and drink, and goes on the nest for protection from the light. The general appearance is deceptive as indicative of the diseased condition. Upon investigation and when an effort is made to see the bird walk, it may not rise to the feet, or moves the feet with difficulty, staggers, with the head wabbling and turned backwards which attitude appears to be involuntary. With the development of the disease, paresis oceurs and stupor becomes deeper. In some cases in this stage a viscid secretion flows from the nostrils and mouth or the material may be squeezed from the nostrils. A diphtheritic type has been observed, in which fibrinous exudate occurs in the nasal, oral and pharyngeal cavities. Conjunctivitis and lachrymation may occur.

As a rule there is no diarrhea. The feces are quite solid, often green in color and of the usual amount. In the later stages of the disease some birds discharge hardly any feces. It death and after, a fluid is discharged from the eloaca and beak.

Morbid anatomy. Freese has conducted numerous antopsies 
of naturally infected birds and describes the lesions as follows:

Hemorrhages in the mucous membrane of the passage between the proventriculus and gizzard are highly characteristic of the disease. They also occur in the mucosa of the true proventriculus between the conical elevations or very seldom they are limited only to these elevations. It is possible for the hemorrhages to occur in both places in the same fowl. The hemorrhages of the mucosa between the papillæ or in the opening between the two stomachs may be superficial, in the mucosa or in the tissues lying beneath. They vary greatly in size and may be barely visible or several centimeters in diameter. They are red or dark red in color and often are not sharply circumscribed. If the conical papillæ are involved, either the whole mass is reddened or only the upper border about the mouth of the gland. The hemorrhages in the proventriculus in many cases are very small and are often quite separated, so that their presence might not be observed on superficial examination. This is all the more likely unless the viscid, gray, white, cloudy mucus is removed.

Small hemorrhages are frequently found in the fatty tissue surrounding the gizzard, on the mesentery of the small intestine and superficially under the cuticle of the gizzard. More rarely, fine subepicardial hemorrhages are observed in the pericardium, especially in the heart fat, and in the tracheal, laryngeal and pharyngeal mucosæ. They are rarely found in the pulmonary pleuræ.

The kidneys are swollen to an extraordinary extent. They are either grayish brown in color and clouded or in the acute cases, dark brown on account of the presence of an excessive amount of blood.

In the functional ovary, there is a marked congestion of the vessels of the yolk capsules. Occasionally hemorrhages are observed under the serosa in the yolk mass.

In acute cases a certain degree of swelling of the spleen is observed. As a rule, however, lesions of this organ do not occur. The liver does not show important lesions, but some writers describe congestion in acute cases.

Inflammation of the small intestine is at the most limited to the first portion of its length. In some cases there is observed only a slight catarrhal inflammation, while in other cases there is a marked inflammation with hemorrhages. The ceca and the remaining large intestine are very rarely inflamed. Inflammation of the intestine occurs in about half of the cases. 
Exudate in the aldominal cavity is either serous or sero-fibrinous. The fibrin as a rule is in suspension in the serous thuid in the form of flakes, or is deposited loose on the peritoneum in the form of threads or sheets.

The peritoneum is not inflamed but is smooth, shiny and transparent. Exudate oceurs in the abdominal eavity in about a quarter of the cases.

Exudate occurs in the pericardium in the form of a serous or sero-fibrinous fluid, which on opening of the pericardial sac often coagulates into a gelatinous mass. The presence of exudate in the pericardium occurs somewhat less often than in the abdominal cavity.

A bluish red coloration of the comb occurs very often. There is nothing characteristic about it as compared with a number of other diseases.

Some authors emphasize the significance of catarrh of the upper air passages and of the pharynx. They note the occurrence of viscid gray white mueus in the beak, throat and nasal cavity, but it is considered to be a normal secretion which remains in its place of production for some time before death.

In wild ducks dying of the nervous type it has been noted that autopsy reveals no characteristic gross changes.

Diagnosis. The presence of hemorrhages in the proventriculus, the swelling of the kidneys and the severe injection of the blood ressels in the yolk capsules of the ovary, oceasionally associated with the presence of hemorrhages, in birds dying of a pest-like disease, are most significant. The autopsy findings alone will not alwars suffice for making a diagnosis. In some cases as in other infectious diseases, the lesions may be so slight as not to warrant drawing conclusions. A diagnosis of fowl plague can be established most certainly by successful transmission to a hen with absence of a causative agent recognizable in tissues or in cultures from the blood and organs.

Differential diagnosis. Phosphorus poisoning canses lesions having the greatest similarity to those of fowl pest and also causes a high mortality. In general the hemorrhages in the proventrieulus necurring in phosphorus poisoning extend deeper tham in forl pest, also in the poisoning, erosions occur in the hemorrhagic mucosa. In phosphorus poisoning there is also a marked inflammation of the upper part of the intestine. Notwithstanding the great similarity of the lesions the two conditions can be differentiated at antopsy by 
the escape of the so-called phosphorus vapor from an opening in the gizzard or crop and by the characteristic smell.

Next in similarity is fowl cholera. This is characterized by the occurrence of subepicardial hemorrhages, an exudate in the pericardium, a marked inflammation of the intestine and by the inflammation frequently cceurring in the lungs. In only a few cases would these lesions be confounded with those of fowl pest.

Rabbit inoculation is valuable, for this animal is immune to fowl pest and susceptible to fowl cholera. Spirochetosis will be suggested by the drowsiness and paralytic symptoms and must be differentiated by microscopic examination of blood taken during an early stage. In spirochetosis such blood will be found to contain spircchetes, but such an examination will be misleading, if the sample be drawn after the crisis has occurred.

Preventive measures. Treatment is unarailing. The general sanitary measures such as recommended for cholera, should be employed.

Attempts to immunize by raccination with diluted red corpuscles have failed. I slight measure of success by vaccination with virus killed by ether, has been obtained. Birds after two vaccinations were protected to a slight extent in that death was delayed.

The agency of poultry exhibitions in rapidly disseminating the infection should be borne in mind. The disease apparently gained a wide distribution in Germany as a result of a show held in Brunswick in 1901.

\section{REFERENCES}

1. Belfanti and Ascoli. Spigolature nella Peste Aviaria e nell' Afta. Clin. Vet., Vol. 39, 1916, p. 577.

2. Centanni. Die Vogelpest; Beitrag zu dem durch Kerzen filtrirbaren Virus. Centralbl. f. Bakteriol. (Etc.), 1 Abt. Orig., Bd. 31, 1902, S. 145.

3. Cominotti. Peste ariaria nella anitre. Clin. Vet., Vol. 39, 1916, p. 129.

4. Dubois. Une maladie infectieuse des poules à microbes invisibles. Compt. rend. Soc. de biol., Vol. 54, 1902, p. 1162.

5. Freese. Ueber Hühnerpest mit besonderer Berücksichtung der pathologischen Anatomie. Deutsche tierärztl. Wchenschr., Bd. 16, 1908, S. 173.

6. Hertel. Ueber Geflügel-cholera und Hühnerpest. Arb. a. $d . k$. Gsndhtsamte., Bd. 20, 1904, S. 453.

7. Joest. Beitrag zur Kenntnis der Bakterienflora des Hühnerdarmes nebst einigen Bermerkungen über eine neue Hühnerseuche. Berl. tierärztl. Wehnschr., 1902, S. 241. 
8. Kleine und MLellers. Ueber Hühnerpest bei Gänsen. Centralbl. f. Bakteriol., Bd. 39, 1905, S. 545.

9. Iraus und Schiffmann. Studien über Immunisierung gegen das Virus der Hühnerpest. Centralbl. f. Bakteriol. (Etc.), 1. Abt. Orig., Bd. 43,1907 , S. 825 .

10. Landsteiner u. Berliner. Ueber die Kultivierung des Virus der Hühnerpest. Centralbl. f. Balteriol. (Etc.), 1 Abt. Orig., Bd. 67, 1912, S. 165 .

11. Leclainche. La Peste Aviaire. Revue Gén. de. Méd. Tét., T. 3, 1904 , p. 49.

12. Lipschütz. Ueber mikroskopisch sichtbare filtierbare Virusarten. Centralbl. f. Baliteriol., Bd. 48, S. 77.

13. Lode und Gruber. Bakteriologische Studien über die Aetiologie einer epidemischen Erkrankungen der Hühner in Tirol. Centralbl. f. Bakteriol. (Etc.), 1 Abt., Orig., Bd. 30, 1901, S. 593.

14. Maggiora und Valenti. Ueber eine Seuche von exudativen Typhus bei Hühnern. Zeit. f. Hyg., Bd. 42, 1903, S. 185.

15. Mane. Immunizierungsversuche bei Hühnerpest. Arb. a. d. $k$. Gsndhtsamte, Bd. 21, 1904, S. 537.

16. Ostertag und Bugge. Weitere Untersuchungen über die Hühnerpest. Zeitschr. f. Infektionskr. d. Haustiere, Bd. 2, 1906, S. 1.

17. Ostertag und Wolffhügel. Untersuchungen über die "Hühnerpest," die neue Geflugelseuche. MIonatschr. f. prakt. Tierh., Bd. 14, 1903, S. 49.

18. Ottolenghi. Ueber einen besonderen Befund bei der Geflügelpest. Centralbl. f. Bakteriol. (Etc.), 1 Abt. Orig., Bd. 67, S. 510.

19. Russ. Beobachtungen über das Virus der Hühnerpest. A $r$ ch。 $f$. Hyg., Bd. 59, 1906, S. 286. 


\section{CHAPTER VIII}

\section{AVIAN TUBERCULOSIS}

Characterization. Avian tuberculosis constitutes an affection closely related to the same clisease in mammals, characterized by the development of tubercles principally in the visceral organs.

Poultrymen refer to the disease as spotted liver, liver complaint, rheumatism, or describe the birds as going light.

Species affected. A considerable number of domesticated and wild birds are affected. The disease has been observed in the fowl, guinea-fowl, peafowl, turkey, duck, goose, swan, pigeon, ostrich, parrot, canary, pheasant, sparrow, and in a large number of species of wild birds kept in zoological gardens.

Etiology. Typical strains of the avian variety of $B$. tuberculosis possess characteristics enabling them to be differentiated from the human and bovine varieties. Avian bacilli are more readily isolated and eultivated in pure culture, than are the mammalian varieties. The avian organisms are somewhat shorter and stain more evenly than those of the mammalian variety. While the guinea pig is most susceptible to mammalian strains, inoculation with avian material may result in emaciation with no visible lesions, or in relatively slight lesions of a non-progressive character. When lesions are not visible after inoculation, it does not preclude the possibility that tuberele bacilli may be present and demonstrable microscopically, or culturally, or by further inoculation of the tissue into other guinea pigs.

The transmissibility of avian tuberculosis to mammals and vice versa is of interest in connection with the control of the disease. As a general rule, fowls are not susceptible to the human and bovine varieties. Experimental evidence exists to show that calves may be infected by injections of avian tubercle bacilli, and that fowls have been artificially infected with tubercle bacilli of mammalian origin. The majority of experiments to test this transmission have given negative results. In view of these findings, the simultaneous occurrence of tuberculosis in eattle or man, and in forvls exposed to infection from them, has slight value as evidence of transmission. All three varieties of the disease are so 
common that coincidences may occur. The literature of tuberculosis reveals comparatively few instances in which avian tuberele bacilli have been found naturally in cattle or in man.

With the exception of the intertransmissibility of avian tuberculosis between pigs and fowls, the disease in fowls stands by itself as an economic and hygienic problem. There is considerable evidence that parrots and canaries may be infected from a mammalian source.

While pigs undoubtedly contract tubereulosis most frequently from bovine sources, they do to a lesser extent acquire it from avian sourees. The possibility of the transmission of tubereulosis between pigs and forls should not be overlooked in comnection with hygienic measures against the disease in either. Outbreaks of avian tuberculosis in pigs in contact with infected fowls have been observed both in Denmark and in the United States. Eastwood and Griffith made cultures from a series of seventy-eight pigs exhibiting only localized tuberenlosis. The avian tuberele bacillus was found alone in twenty-six of these pigs, or thirty-four per cent. Of the remainder, the avian organism was found to be present along with bovine bacilli in two cases.

Symptoms. I'ntil the disease has developed sufficiently to canse emaciation, there are no characteristic symptoms, except in the rare cases when the skin or joints are involved. In the latter case poultrrmen describe the condition as rheumatism. This term as applied to a disease in birds is practically synonymous with arthritis. Anemia and emaciation are constant in advanced cases, the latter becoming extremely marked. The loss of weight is very noticeable; the bird becomes weak and moves about very little. This disinclination to move may be due either to extreme weakness or, more rarely, to involvement of the joints. Under such conditions, the bird spends much of the time in a crouching position. An unnatural character of the gait is noticeable.

The eyes are bright and appetite remains markedly good in advanced cases. The skin and visible mucosæ are pale, and the feathers are ruftled. The temperature is usually within the normal range, and rarely is subnormal. Young birds affected with the disease enough to show stmptoms are not eneountered, doubtless due to the time necessary for the development of serious lesions.

The separation of deaths from one another in point of time is a characteristic feature of the disease.

Morkid anatomy. Emaciation is striking in most calses that 
die of the disease. The muscles of the breast may be represented by barely a trace.

Avian tubercles do not differ greatly in gross appearance from those of the mammalian type. The smallest ones consist of nodules of tissue in which central caseation has not yet begun. In larger ones varying degree of caseation is illustrated, but calcification is not frequently observed. Avian tubereulosis differs from mammalian tuberculosis markedly in that the visceral organs primarily are involved and lesions of lymph nodes are of subordinate importance.

The liver is usually studded throughout with nodules which are easily separated from the hepatic tissue. There may be several sizes of nodules, suggesting that several corresponding invasions of bacteria have occurred. The smaller tubercles are white or grayish white, while the larger, older ones are more yellow in color. The presence of the tubercles brings about secondary degenerative changes in the liver, as a result of which the tissue becomes pale and friable. As a consequence, fatal hemorrhages may occur. The organ is enlarged in advanced cases, and in exceptional cases may constitute one-fourth of the weight of the bird.

The spleen, like the liver, is found involved in practically all advanced cases. The relative enlargement is greater, for this organ may attain the size of a walnut. The small amount of functional tissue remaining is often striking.

The smallest tubercles of the intestine are located in the deeper layers of the mucous membrane. Slightly larger ones are in intimate contact with the intestinal wall but project somewhat. Still larger ones, as much as an inch in diameter, are pedunculated and in most cases the interior of such tubercles communicates with the lumen of the intestine. Lesions of the intestine also occur in the form of numerous but isolated ulcers on the mucous membrane.

The kidneys, ovary, mesentery, and lungs are involved less often, while cases of tuberculosis of the pancreas, gizzard, and skin are rare in fowls. In the kidneys, the tubercles are discrete, hard, and glassy. These organs alone may be affected. Small tubercles are observed on the mesentery associated with tubercles on the intestine. Involvement of the lung apparently is associated with advanced development of the disease and then it occurs comparatively rarely. The tubercles may be isolated or aggregated into extensive areas. Skin lesions may consist of single spherical masses, each surrounding the root of a feather, or more often, consist of larger masses. 
A form of nodular discase of the skin has been observed in which tubercle-like organisms are found, and efforts to cultivate them give negative results. Another point of difference is that the organisms are decolorized by acidulated alcohol, which does not oceur in the case of true avian tubercle bacilli. Unquestionable tuberculous lesions of the skin occur in pigeons. A tuberculons lesion consisting of a cutaneous horn is relatively common in parrots.

Bone lesions have been observed particularly on the breast bone and ribs. Lesions oceur in the knee, back, digital and shoulder joints. The extremities of the bones enlarge with accumulation of cheesy material, or may discharge a semi-fluid substance.

Differential diagnosis. A diagnosis of tubereulosis by physical examination of the living bird is hardly possible in most cases, nor is this important. Emaciation creates a strong presumption of the existence of tuberculosis but is not conclusive, for it occurs in asthenia, in serious infestation with air sac mites and in other conditions less well understood.

At autopsy of fowls, the possibility of the occurrence of tumors of the liver, and nodular treniasis of the intestines should be borne in mind. Nodular lesions not involving the liver and spleen, may quite safely be considered as not tuberculosis. In the turkey the more highly colored areas occurring in infectious entero hepatitis are not likely to mislead one familiar with these lesions. Gouty arthritis and bumblefoot in the fowl are suggestive of tuberculosis. Nodules in the lung caused by aspergillosis may be differentiated from tuberculosis by microscopic examination.

Microscopic examination of smears for tubercle bacilli is available as a means of diagnosis, especially since organisms are unusually abundant in lesions of avian tuberculosis.

Tuberculin test. Numerous efforts have been made to apply the subcutaneous tuberculin test to fowls, using tuberculin prepared from both avian and mammalian cultures, but without success.

Better results have attended trials with the intradermal (intracutaneous) form of the test in manner similar to that in which it is applied to cattle. The wattle is chosen as the site of injection and a very fine needle of No. 26 or 27 gauge is employed. The aim is to deposit the tuberculin not in the deeper layers, but in the stratum Malpighii. It should not be injected so superficially that the tuberculin will be lost by rupture of the epithelium.

The tubereulin employed is prepared in the usual mamner from an avian tubercle culture, and is diluted fifty per cent for use. The 
dose varies from one-twentieth to one-thirtieth of a cubic centimeter. After short experience, attention to the graduations on the syringe will be abandoned and the dose judged by the appearance of the tissues at the point of injection. The head of the fowl should be held with considerable firmuess by an assistant during the injection, in order to obviate the necessity for inflicting unnecessary damage to the tissues, which would interfere with observing the results. Care must be taken to avoid injury of the wattle by rough handling.

A positive reaction to the test consists of a swelling of the injected wattle. Perhaps ten per cent of the swellings will be so slight as to be doubtful. Observations made by Van Es and Schalk indicate that among the reactions considered by them as doubtful, about one-half were found on slaughter, to contain lesions, and half to be free from lesions. The majority of reactions will leave no doubt as to the existence of a pronounced edematous swelling, which may increase the thickness of the wattle to several times normal.

Of 90 birds tested by Van Es and Schalk, showing typical reactions, 85 were found tuberenlous at slaughter and 2 showed no visible lesions. Of 130 birds slaughtered and found without lesions, 120 or 91.5 per cent had failed to react to the test. About 8.5 per cent of the non-reacting fowls were found to have lesions. Thus the results shown indicate that the intradermal tuberculin test for fowls is about as accurate as the same test or the subcutaneous test in cattle.

The test surely furnishes information regarding the extent to which infection has spread in a flock and consequently provides information regarding which birds should be eliminated. Of three and four year old birds in an infected flock, as high as 86 per cent may react. Of two year olds, 24 per cent and of pullets, 3 per cent have reacted. It may further be of use in determining whether or not tuberculosis exists in a flock from which it is contemplated to purchase birds.

Transmission. The tendency of lesions involving the intestine to perforate the wall and discharge into the lumen, provides the main channel for the dissemination of tubercle bacilli. Microscopic examination of such lesions reveals enormous numbers of the organisms. In view of the rarity of pulmonary lesions and the relative frequency with which intestinal lesions oceur, the feces must be regarded as the chief channel of elimination of infection. Tubercle bacilli have been demonstrated to be present in the feces of infected 
birds but are not found in the feces of a very lare percentage of such birds.

Tuberele bacilli have been demonstrated in egos laid by tubereulous hens but it has not been demonstrated that such a chamnel of dissemination of the infection is an important one. It seems quite certain that the disease is not observed in young birds ats might be expected if transmission through the egg were common.

Tubercle bacilli, when directly exposed, are sensitive to sunlight, but the conditions about poultry establishments are such as to warrant belief that they may persist alive for months.

Feeding experiments leave no doubt but that the alimentary tract is the chief portal for entrance of infective material. Exposure of healthy birds by contact with infected birds sets up disease which may persist for a year before important lesions develop. The eating of carcasses of birds dead of the disease undoubtedly contributes to dissemination of infection. In some cases, exposure ly feeding infected droppings has induced disease that was fatal in six months.

While the introduction of tuberculous fowls must be regarded as the most common means of infecting a flock, there exists the possibility of its being accomplished by infected pigeons, among which the disease is not uncommon, or by pigs.

Economic importance. Tubereulosis is capable under farorable conditions of causing a very heavy death rate during the course of a year. Comparatively few accurate observations have been made in this country, but annual losses up to nearly 50 per cent have been reported.

Enongh reports are available from the northern half of the United States, the Pacific Coast, and Canada to indicate that the discase is prevalent in the area mentioned.

The insidious nature of the disease contributes to the unconcern of the owner of an infected flock. Deaths will not occur until the flock has been extensively infected and when they occur, are so scattered in point of time that they do not cause the same concern on the part of the owner as would be occasioned by the same number occurring in a short time. Consequently there is lack of incentive to take up repressive measures.

Prevention. The problem of the control of tuberculosis in fowls presents several features that render it much simpler than the corresponding problem in cattle. The productive uscfulness of hens is ended at three years of age, when it is the common practice 
to market the birds for table use, if this indeed is not done after two years. Thus total replacement of stock is possible in a short period. The extensive use of incubators and brooders assures the isolation of the young from infected old birds. Where the business is conducted on an intensive scale on a small area, isolation of birds of various ages is quite often practiced. Thus ideal conditions for combating the disease already exist in certain plants, if indeed the disease does ever make considerable progress under such conditions. It is undoubtedly true that the disease only becomes serious when there is unlimited opportunity for mingling of birds of various ages as exists where poultry are kept without restraint. This is the condition in the average small poultry yard, or in an extensive poultry business where the system of using unfenced colonies is practiced.

In attempting to control the disease among unfenced birds, the end sought is to secure isolation of young birds and maintain it throughout life.

In every extensively infected flock there will be a certain number of badly diseased, worthless birds, which may be recognized by physical means, and destroyed. The intradermal test may be applied to determine the extent of the infection. The results of this test, the age of the birds and egg yield, will aid in reaching a decision as to whether all or part should be marketed at once. Many reacting birds will be found at autopsy, to contain very minute lesions and there should be no prejudice against killing such for food.

Appropriate disinfection of contaminated buildings, and temporary abandonment of areas of soil too large to disinfect, will naturally suggest themselves.

Van Es reports upon results of testing a flock of 249 birds, of which 43.37 per cent were tuberculous. All reacting and undesirable birds were eliminated, leaving 56 non-reacting birds. These, together with 47 birds purchased after the test, increased the next year to 249. One bird died of tuberculosis after the first test and had undoubtedly failed to react on account of severe lesions. A second test made a year after the first one revealed 2.41 per cent of reactors. These were regarded as having contracted the disease from the one non-reactor of the first test.

Mention of hygienic measures such as good ventilation, sunlight, and warm quarters has no place in a discussion of the control of tuberculosis in fowls. The disease is a veritable scourge in parts of California where such hygienic conditions are most favorable. 
Wild birds. Deaths from tubereulosis are frequent among wild birds kept in captivity. Out of 459 birds dying in the Berlin Zoological Gardens during a given period, 118 or 25.7 per cent were tuberculous. The disease was present in specimens belonging to 10 of the 15 orders of birds represented. The other five orders were represented by few specimens, and the observers, Koch and Rabinowitsch, believe that all orders of birds are susceptible. A similar examination of 500 birds from the London Zoological Garden revealed tuberculosis in 30 per cent of the birds dying.

\section{REFERENCES}

1. Hastings and Halpin. Avian tuberculosis. Univ. of Wis. Agr. Exp. Sta. Research Bull. 28, 1913.

2. Moore. The morbid anatomy and etiology of avian tuberculosis. J. Med. Research, n. s. Vol. 6, 1904, p. 521.

3. Van Es and Schalk. Avian tuberculosis. North Dakota Agr. Exp. Sta. Bull. 108.

4. Ward. Tuberculosis in fowls. Univ. of Cal. Agr. Exp. Sta. Bull. $161,1904$. 


\section{CHAPTER IX}

\section{AVIAN DIPHTHERIA AND BIRD POX}

General discussion. The group of pathological conditions referred to by various authors under such designations as simple catarrh, colds, contagious catarrh, influenza, coryza, roup, canker, diphtheria and bird pox present a complicated subject for discussion.

The pathological features presented by the lesions of the mucosa of the fowl's head in the conditions described as roup or diphtheria, have been concisely summarized by Moore. He studied the disease in the eastern United States and has described three stages or varieties of lesions as follows:

"(1) An exudate of a serous or muco-purulent character in the conjunctiva or nasal cavities. Ordinarily this condition cannot be recognized in the mouth. The mucosa in these cases is apparently but slightly altered.

"(2) The mucosa over a small or larger area is covered with a spreading exudate of a grayish or yellow color. It is firmly attached to the mucous membrane and when removed leaves a raw, bleeding surface. Sections through this exudate and the subadjacent tissues show that the epithelial layer is destroyed, and the underlying tissue infiltrated with cells. The extent of the infiltration varies in different individuals.

"(3) The mucosa is covered with a thick mass of exudate, varying in color from a milky white to a lemon yellow or brown. It is easily removed, leaving a more or less granular and healed surface. This sloughed mass is frequently dried at its margins to the adjacent tissue. It emits a strong putrid odor, due to decomposition. The drying of the margins prevents the fowl from expelling the exudate after it becomes separated from the underlying tissue."

In the far West and South, the lesions described will commonly be found accompanied by the tumor-like lesions known as chicken pox or epithelioma contagiosum.

The earlier studies of the etiology of the diphtheritic lesions of fowls extending over a long period have resulted in a mass of conclusions, in the main contradictory and confusing. Various authors have ascribed the lesions as due to gregarina, chlamdrozoa, coccidia and numerous species of bacteria.

The discovery that the filterable virus of chicken pox is capable of causing diphtheritic lesions has now received abundant confirma- 
tion and furnishes a basis for clearing up the confusion. Among writers who have studied both avian diphtheria and pox and who agree on this point are Uhlenhuth and Mantenfel, Schmid, Bertegh, and Sigwart.

Whether there are other specific infections diseases of fowls characterized by diphtheritic lesions not due to pox virus, remains to be determined by further investigation. The idea has been advanced that diphtheritic lesions of the mueosa of the head of the fowl may result from invasion by various bacteria.

There is ground for questioning the validity of differentiating into separate disease entities such conditions as simple catarrh, common colds, contagious catarrh, influenza and coryza. In large infected flocks, birds with eatarrhal lesions alone, diphtheritic lesions alone, and pox lesions alone will be found in close association with others perhaps exhibiting all these lesions. Clinical evidence in such flocks points very strongly to the conclusion that the cases characterized by the presence of serous exudate in the nostrils are but the early stages of avian diphtheria. Insufficient etiological evidence is available to justify recognizing such conditions as specific infections diseases.

Characterization. Avian diphtheria is an infectious disease, manifested by the formation of catarrhal exudate in the nasal cavity, eyes, and simuses associated with pseudo-membranes in the mouth and wart-like nodules on the external surface of the head. Until recent years, the term diphtheria or roup was applied to cases showing the internal exudative lesions, while those with external growths were designated bird pox, chicken pox or epithelioma contagiosum. The discovery that a single cansative agent can produce both types has resulted in the two conditions being elassed as simply external and internal lesions of one disease.

Etiology. Diphtheria and bird pox are caused by a virus the nature of which is not well known. The organism has not been certainly identified microscopically and is so small that it will pass through a Berkefeld filter. However, according to Marx and Sticker, it will not pass through a porcelain filter.

Widely diverging opinions concerning the nature of the virus have been expressed by the varions writers who have studied the lisease. Friedberger and Fröhner assigned protozoa of the elass gregarina as the cause. Borrel described certain cell inclusions for which Lipschütz has suggested the name Strongyloplasma avium. Halasi believes the etiologieal agent to be a chlamydozoon. 
Tenacity of virus. Burnet observes that finely powdered virus in physiological salt solution placed in sealed ampoules in a water bath at $60^{\circ} \mathrm{C}$. is killed in about 8 minutes. At $56^{\circ} \mathrm{C}$. the finely powdered virus does not resist longer than half an hour. In suspension in water at $37^{\circ} \mathrm{C}$. it has been found inactive after 8 days; active after 3 days; active after 30 days at $22^{\circ} \mathrm{C}$.; active after 6 days at $25^{\circ} \mathrm{C}$. Marx and Sticker found virus active after 3 hours at $60^{\circ} \mathrm{C}$., but the material probably was not as finely powdered as in the experiment conducted by Burnett. The same authors found that desiccated virus in ampoules deprived of air remained active after an hour at $100^{\circ} \mathrm{C}$. Scabs exposed to the sunlight in a Petri dish were still virulent after two months. Reischauer observes that the virus resists dry heat at $80^{\circ} \mathrm{C}$. for 15 to 30 minutes and moist heat 5 minutes at $100^{\circ} \mathrm{C}$. M Tarx and Sticker found a suspension of crusts virulent after $1 \frac{1}{2}$ hours in a 1 per cent solution of carbolic acid. In solutions of 2 and $21 / 2$ per cent it lost its virulence. Reischauer notes that the virus is killed in 5 minutes by 1 per cent solution of potash, acetic acid or carbolic acid. Burnett kept a suspension of virus in ordinary water in a refrigerator at $6^{\circ} \mathrm{C}$. for 60 days and found it virulent after that length of time. Finely powdered virus mixed with glycerine was found to be virulent after 120 days.

Haring and Kofoid tested the effect of various germicides upon powdered pox virus. The virus was saturated with the germicide and after the lapse of a certain amount of time, the virus was inoculated into a fowl by scarification. Five per cent carbolic acid failed to kill in 20 minutes; 2 per cent liquor cresolis compositus failed to kill in 20 minutes; 2 per cent potassium permanganate failed to kill in 20 minutes; 2 per cent copper sulphate failed to kill in 20 minutes; tincture of iodine failed to kill in 10 minutes; mercuric chloride in 1: 1000 solution failed to kill in 20 minutes; steam heat at $100^{\circ} \mathrm{C}$. failed to kill the virus in 5 minutes but did do so in 30 minutes; dry heat at $200^{\circ} \mathrm{C}$. killed the virus in 30 minutes. Powdered pox scabs saturated with physiologic salt solution and allowed to decompose for 10 days in a warm place proved non-virulent. Mry powdered pox scabs kept in a test tube from May, 1906, to September, 1911, proved virulent.

Pathogenicity. Diphtheria and pox are especially prevalent in chickens and pigeons, and are also common in turkeys, geese, ducks and guinea-fowl. Pheasants, quail and various wild birds are susceptible. 
Natural infection. Natural infection occurs under conditions which are not yet well understood. Apparently, the virus penetrates through a slight lesion of the skin or mucous membranes and reaches the skin or the mucous membranes of the mouth by way of the blood stream. Infection by ingestion probably oceurs. An instance of infection by a biting fly, Stomoxysis calritrans, has been reported.

Experimental transmission. The infection may be readily passed from the infected to susceptible birls by rubling infective material into the skin or mucous membrane of the latter, especially if the parts be slightly scarified.

In the pigeon the disease occurs on feathered portions of the body. To secure infection it is only necessary to pull out the feathers and rub in the virus lightly. After five or six days the skin shows a characteristic swelling. The follicles are greatly enlarged and upon pressure, whitish plugs are forced out.

The virus is readily transmitted from the pigeon to the fowl but is transmitted from the fowl to the pigeon with great difficulty, if at all. Passage through the fowl appears to lower the virulence of the virus for the pigeon.

Intravenous injection of the fowl with pox virus as a rule causes only diphtheritic lesions of the mucosa of the head, with no changes of the skin. This is true whether the virus which was injected, originated from the skin or the mucous membrane lesions.

Inoculation of the comb with material from mucous membrane lesions does not always result in the occurrence of pox lesions. Some observers interpret these negative results as proving the absence of pox virus in the diphtheritic material. Others regard the results as mere failures of pox virus to take.

The virus is present in the circulating blood and internal organs in acute cases, as demonstrated by inoculation experiments. In some cases injection of liver and blood of birds affected with chronic lesions of the mucous membranes of the head, will reproduce the disease. When positive results occur, the incubation period is long and the cases induced are mild. In chronic cases, the virus in the blood appears to be weakened or lessened in amount. The virus from either skin or mucous membrane lesions, when filtered, will give positive results in most cases. Even if the material filtered is of high virulence, it will only produce mild cases of mucous membrane infection.

The incubation period for both diphtheritic and pox lesions varies 
from 3 to 12 days, depending upon the virulence of the virus and the method of inoculation. After injection of filtered virus, the period varies from 6 to 15 days. Isolated instances of incubation periods to 25 days have been observed.

Mortality. The fatalities occurring in an infected flock vary within a wide range depending upon individual circumstances. Under farorable conditions, the disease may not spread to more than 10 per cent of exposed birds, with the death rate proportionately low, while in other cases 80 per cent of the exposed may become infected, with deaths relatively numerous, approaching in some instances 50 per cent of those affected. The virulence of the virus is variable and constitutes the main element for consideration in each outbreak. The mortality depends, to a large extent, on climatic conditions. Fowls are more severely affected during the fall and winter months and it is during this period that the heaviest losses are encountered.

Course of disease. Arian diphtheria may follow an extremely acute course, affected birds dying without showing noticeable external symptoms which would attract the casual observer. In less acute cases the disease may be resisted for several days after symptoms are apparent, the fowl finally succumbing apparently as a result of toxemia, or of starvation due to eye affection which inhibits feeding. In' the milder, and especially in the more chronic eases, marked symptoms may persist for several weeks and finally result in recovery, in so far as outward appearances are indicative.

Symptoms. The disease is manifested by three clinical forms: (1) a pure mucous membrane disease, (2) a pure skin disease, and (3) a combination of skin and mucous membrane affection. The disease usnally first manifests itself in a discharge from the nostrils which soon becomes viscid and plugs the nasal passages. In some individuals the first evidence of disease presents itself in the eye. One or both eyes may show a sticky discharge that tends to gum the eyelids and obstruct vision. The exudate continues to form in the conjunctival sac, causing a swollen appearance of the eye and asstming a cheesy character. This, through pressure and the accompanying inflammation, may produce panophthalmia and loss of the eye. The exudate in the nostrils also becomes cheesy in consistency and causes a pressure on the cleft palate and distortion of the bony structures of the adjacent parts. The affected bird early shows extreme dullness and loss of vitality. The tendency is to mope around 
indifferent to its mates, surroundings, or to a person's approach. The head feels warm to the touch and there may be a rise of temperature. Diarrhea is often present. Death may result within two or three days in severe outbreaks or the bird may linger for a week or more with progressively increasing debility and more marked lesions. The head in many cases shows a pronounced swelling on one or both sides below and in front of the eye due to the accumulation of a cheesy mass within the suborbital sinus. Sneezing, which has been apparent in the early stages, gives way to a whistling or rattling in the throat. The inflammation in the nostrils may spread to the mouth and pharynx, or even to the larynx and trachea, where exudates are formed which are principally of the croupous type. Breathing is entirely carried on through the mouth and the continual passage of air over the tongue tends to dry the tip with the result that its surface becomes hard and contracted. This condition is commonly termed "Pip" and may oceur in any atfection which closes the nostrils, and compels breathing through the mouth. Death may be caused by dyspnea from the presence of massive accumulations in or upon the larynx, from toxins absorbed from the primary causative agents and secondary invading organisms, or from starvation, owing to inappetence, or the failure to discover food in blind cases. No doubt the combination of causes acting together are responsible for the heavy mortality, amounting to 90 per cent in some instances, among young birds. Before death, the subject becomes emaciated, sleepy, and finally uneonscious for a period of several hours.

Mild cases show less marked disturbances and may go on exhibiting stmptoms for several weeks, finally apparently reeovering their normal condition. Certain individuals retain the disease in a chronic state and serve as carriers for future outbreaks.

Oftentimes the bird shows no external manifestations of illness. The comb and wattles have a deep red appearance and the hen continues to lay. She dies quite suddenly and, on examination, the only lesion apparent is the formation of a false membrane on the border of the larynx or just inside that organ. This exudate has occluded the air passage and caused death by suffocation. Frequently the condition is discovered in time to save the fowl through the owner's attention being attracted by a peculiar noise which accompanies the effort of the bird to breathe. It will be observed to open its mouth widely and attempt to dislodge the exudate he a spasmodic movement of the respiratory apparatus. The resulting ef- 
fect may be termed a "bird cough." Prompt attention in such cases nearly always saves the life of the fowl.

The disease spreads rapidly through a flock affecting a large percentage of the birds and causing the death of a variable percentage

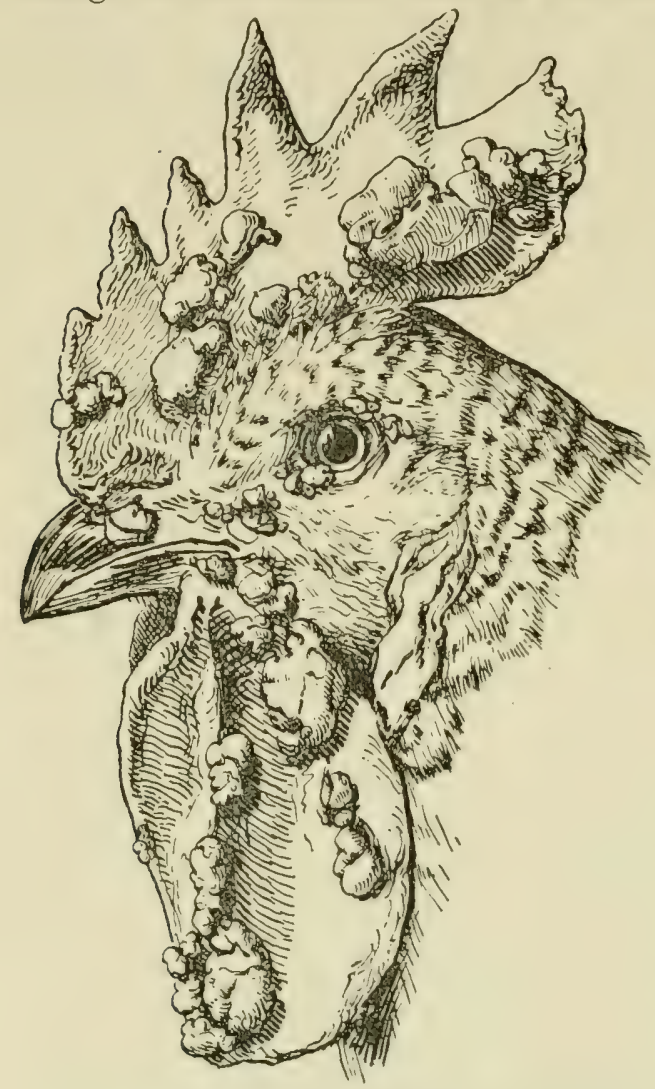

Fig. 8. Head of a Plymouth Rock cock affected with epithelioma contagiosum (bird pox). (Klee) according to the virulence of the virus. Young birds are especially susceptible, adults less so, and a few exhibit complete resistance.

Morbid anatomy. The external lesions have the appearance of wart-like growths prominently raised from the surface of the skin. The size may vary to a considerable degree according to location, or through combination of several nodules. Generally they have a diameter of $1 / 8$ to $1 / 4$ of an inch. These pox tumors occur chiefly on the unfeathered or lightly feathered portions of the body, and particularly on the comb, wattles, eyelids and at the commissures of the beak. They may also be found at times on the under surface of the wings, particularly if there has been an abrasion or bruise at this point. They may be rarely observed around the vent. The nodules first appear as small whitish points which rapidly increase in size, reaching their maximum size in four to six days. They form an integral part of the epithelium, being in reality an extrusion of enlarged epithelial cells. If the superficial scale of exudate is removed, the tumor will be seen to consist of whitish cylindrical masses arranged perpendicularly to the skin. The mass assumes a yellowish color which later changes to a dark brown or black as the tuberosity degenerates and develops 
into a hard dry scab. After seven to nine days this scab may be easily removed leaving a whitish area only slightly clevated above the surface and usually pitted. The scabs retain the virus of the disease and when rubbed on the scarified skin or mucous membrane

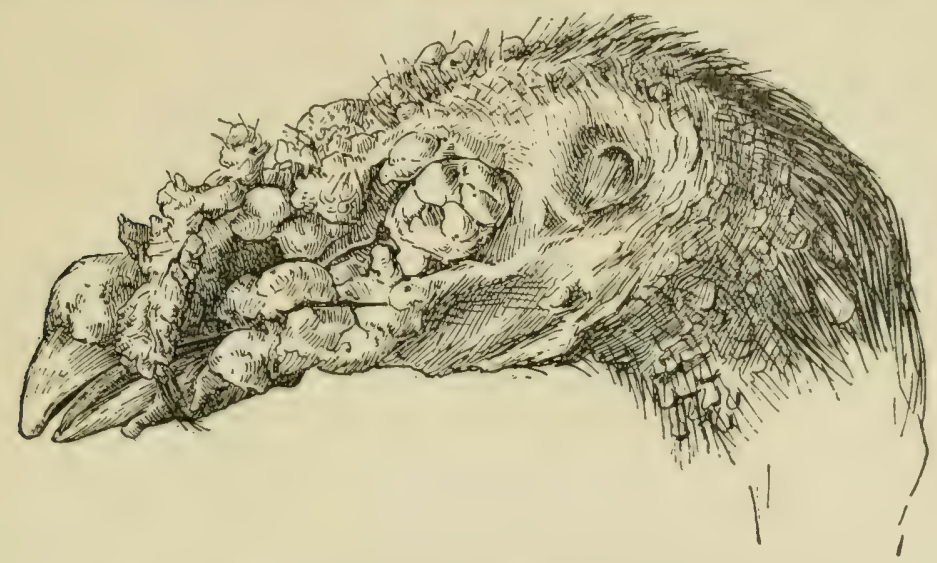

FIG. 9. Head of a turkey affected with epithelioma contagiosum (bird pox). (Klee)

of the mouth, even after a period of five years, may reproduce typical lesions.

Haring and Kofoid describe the microscopic structure of chicken pox tissue as follows:

"The epithelial tumors are produced by a hyperplasia of the epithelium due to an increase both in the size and number of cells. The zone of growth is in the stratum of Malpighii, the principal region of proliferation being in the outer edges from which the cells increase in both directions. A proliferating epithelium forms cell nests surrounded by thick bands of connective tissue, which contain blood vessels with thickened walls. The rapidly proliferating peripheral cells of the Malpighian layer are seen thickly studded with granules. The nucleus contains deeply staining chromatin bodies which are in an active process of proliferation. Karyokinetic figures are common in this region. Nore deenly in the epithelial tumor mass the cells are greatly increased in size and have relatively fewer granules. The nuclei are nale and show little evidence of cell division. In these cells, are large cell inclusions which are very striking in appearance, and which Reischauer and others liave thought to be protozoa. There is usually but one of these bodies in each cell. They vary in size from five to twenty microns, most of them are round, although some of them are quite irregular in shape. They are evidently fatty in structure as they stain black with osmic acid. When eosin and methylene blue are used, they stain a faint pink, resembling somewhat the Negri bodies found in the brain tissue of rabid animals, although they are 
usually much larger. They seem to have no definite internal structure, being usually homogeneous in appearance. Deeper in the epithelial mass the cells are very large and show evidence of degeneration, here the cytoplasm stains poorly and has but few granules. Burnett has called this condition hydropic. In the center of the older epithelial cell masses, the cells are so degenerated that they form a necrotic mass. The nucleus. of the cell first degenerates. The cause of this degeneration is probably due to the fact that the cell nests increase in size by multiplication at the periphery. The outside cells consume all the nutriment at the expense of the central ones and cause their degeneration. The central necrosis rapidly extends, involving the greater part of the tumor mass."

The cell inclusions characteristic of pox have been studied extensively and the interpretation of their nature and significance has been the subject of much discussion. Some observers see in them, stages in the life of a protozoan, while others regard them as products of degeneration of the cell.

In the early stage of false membrane formation, the external layer of the mucosa is principally involved. There is an exfoliation of the cells, which become swollen and amorphous. The cell protoplasm early loses its identity, while the nuclei disintegrate more slowly and appear as independent bodies showing different degrees of degeneration. Mrucous exudates which have undergone coagulation, together with the epithelial cells, enclose leucocytes in various stages of degeneration. An edematous area is formed around the membrane, and wandering leucocytes are observed in the Malpighian layer of the mucosa. During this stage there is a well marked line of differentiation between the morbid area and the comparatively unaffected base. As the pseudo membranes are extended, the underlying tissues are more deeply involved. The deeper epithelial cells become infiltrated and exhibit cloudy swelling. A fibrinous exudate accumulates which holds the cell elements in a compact tenacious mass. In this may be observed numerous organisms, principally of the bacterial type, which are to be considered as secondary invaders. Protozoal organisms may also be present including flagellates, amebas, coceidia and other forms. Some investigators have attributed the origin of avian diphtheria to certain of these protozoa. However, the evidence brought out has not been sufficient to establish these organisms as specific causes. The course of development of filterable viruses in the animal system is a problem which has not been satisfactorily solved, and until this is accomplished, it is unsafe to give etiologic significance to organisms or cell inclusions which may be found associated with certain morbid conditions. 
The gross lesions of the mucous membranes are not characteristic of infection with pox virus alone. Haring and Kofoid have produced similar lesions by mechanical injury or by the injection of progenic bacteria. However, in diphtheritic lesions not due to pox virus, the cell inclusions characteristic of pox infection are not present.

Diagnosis. In trpical outbreaks, the discase is readily recognized by the closely adherent pseudo membranes on the mucosa of the mouth and pharynx, and by the characteristic nodules on the comb and wattles. When the latter are present, the diagnosis is established.

Treatment. This, to a certain extent, must be individual and aceording to the stage of the disease. When the excretions are watery or viseid they may be partially removed from the nostrils by pressing on both sides with the fingers in a manner which will force the material through the openings. The nasal channels are then flushed ont with an antiseptie solution such as permanganate of potash in 2 per cent solution, or boracic acid in 3 per cent solution. This can be accomplished by means of a syringe or medicine dropper. The most. effective method, however, is to submerge the head in the antiseptic solution for a period of 30 seconds, keeping the mouth open and the beak elevated to permit the air to escape from the nostrils and be replaced by the fluid. The treatment should preferably be repeated twice daily.

Certain features of the structure of the fowl's head interfere with natural drainage in diseased conditions, and likewise, interfere with medication. The sub-orbital sinuses so often involved, have no natural drainage. The passages connecting them with the nasal carity lead upward, with the result that fluids cannot escape. The turbinated bones also impede the exit of tenacious exudate. The presence of exudate in both the turbinated bones and in the sub-orbital sinuses prevents access of fluid to the affected mucosa.

When patches are present in the mouth, they should be carefully removed mechanically before treating as above; or the affected areats may be swabbed with tincture of iodine, 2 per eent carbolic acid, in per cent alcohol or argrerol in 20 per cent solution. Swellings of the sides of the head are to be freely opened and the contents remored. The eyes should be kept free of exudate and a mild antiseptic instilled between the eyelids. For this purpose argyrol in 20 per cent solution has given the best results. Two drops are placed beneath the eyelid twice daily. The lachrymal duet and nostrils are also bene- 
fited by the eye injection since the solution passes into them from the eye. In the case of severe eye involvement it may be necessary to feed the fowl artifically because of the difficulty encountered by the bird in finding its food. The question of whether it is profitable to treat all cases rests with the individual owner.

Since the pox tumors disappear in the natural course of the disease, the value of medicinal agents in hastening this end is questioned. Among the remedies that have been recommended are tincture of iodine, an ointment of glycerine, vaseline or lard containing 2 per cent of carbolic acid and 70 per cent alcohol.

Prevention. In some instances infection is brought to a flock by new birds from infected flocks, or by exposure at shows. It is possible that infection is carried on the clothing of persons who have been among infected birds, or by materials on which the excretions of diseased birds have been deposited. Free flying birds may also serve as carriers although it is quite likely that they are rarely responsible for outbreaks. Newly purchased fowls and those which have been exhibited at shows should be quarantined on their arrival for a period of two weeks and carefully examined before being set free among others. When a fowl is observed to be ill with symptoms of diphtheria, it should be isolated immediately. Often it is good policy to destroy the patient because of the danger of the attendant who is treating the bird carrying the virus to healthy fowls. Those handling diseased fowls should disinfect their hands before feeding or caring for others. It would also be advisable to wear rubbers in the hospital and remove them on leaving in order to guard against carrying infection on the shoes.

When infection has broken out in a flock particular eare shonld be exercised in keeping the houses as clean as possible. They should be disinfected by spraying or washing with carbolic acid in 5 per cent solution, compound cresol in 2 per cent solution, or formaldehyde in 2 per cent solution. The yards may be spread with unslaked lime or chloride of lime, or the soil should be plowed under. All birds that die should be burned or buried deep. Those which have recovered, if retaining any evidence of the disease, should be destroyed, as certain ones may serve as carriers and furnish the source of a future outbreak. The drinking water may be medicated with permanganate of potash. A sufficient amount of the latter should be employed to give the water a deep purple color.

Immunization. An attack of the disease confers an immunity complete in from 12 days to 3 weeks but which varies in duration 
and degree according to the severity of the infection. Thus, chronic mild infection of the mucous membrane does not always produce absolute immunity. Infection of the mucous membranes alone will induce an absolute immunity against inoculation of the skin. Various authors have demonstrated immunity in fowls for periods varying from 2 months to 2 years. Live virus only has been shown to confer immunity and the injection of killed virus has no immunizing effect.

A number of workers have attempted the immunization of fowls against diphtheria and chicken pox and treatment of the same conditions by means of a vaceine prepared from the exudates and pox nodules of affected fowls. The material is ground up in a sufficient amount of normal salt solution to form a suspension and is filtered through cotton to remove large particles. It is then diluted until it appears moderately cloudy and heated at $55^{\circ} \mathrm{C}$. for 1 hour. Doses of 1 c.c. are injected subeutaneously on two or three occasions with intervals of 5 to 7 days.

A product prepared by this method has been favorably reported upon as an inmunizing and curative agent by several workers. Experiments by the present writers have not sustained the claims made for the immunizing value of a vaccine prepared by this method. Fowls which have been vaccinated and later artificially inoculated with virulent material have developed characteristic internal and external lesions of diphtheria with accompanying fatalities.

Bacteriology of avian diphtheria. Many investigations of the bacteriology of avian diphtheria were made previous to the recognition of the role of pox virus in causing diphtheritic lesions, so it is not possible to determine whether the lesions were primarily due to pox virus, or were due to bacteria alone. Other reports of investigations conducted more recently do not contain evidence that pox virus was not concerned in the causation of the lesions.

Extensive investigations of the bacteriology of avian diphtheria have been made by Harrison and Streit, Bordet and Fally and by Hausser.

Moore first reported finding a member of the hemorrhagic septicemia group in diphtheritic lesions in fowls, which observation has been confirmed by a large number of investigators. Sigwart has olserved the spontaneous appearance of fowl cholera in birds affected with diphtheritic lesions of the mucous membranes. In these cases the primary cause of the diphtheritic lesions was considered to be pox virus. IIe explains the occurrence by assuming that the 
pox lesions favored the increase of virulence of saprophytic hemorrhagie septicemia organisms commonly present in the mouth.

Jackley has worked on the bacteriology of roup, reaching the following conclusions:

"1. A bacterium, designated culture ' $33 \mathrm{~A}$,' having all of the morphological, cultural and biochemic characters of the pasteurella group, apparently deserves recognition as the etiological factor in roup.

"2. The organism has been recognized by smears in all cases of roup examined.

" 3 . It has been isolated and grown upon artificial culture media and the characteristic local lesions of the disease again reproduced.

"4. Finally, an absolute protection has been shown against the natural disease after immunization with a pure culture bacterin of this organism."

Beach, Lother and Halpin isolated from roup lesions an organism probably belonging to the hemorrhagic septicenia group. Injection of killed culture was shown to produce immunity against injection with virulent culture. The organism was regarded as a secondary invader, as it did not produce lesions of roup or chicken pox. It was regarded as of importance as a secondary invader in wound infection.

The identity in name and similarity of lesions of diphtheria in man and in birds has attracted attention to the possible relationship between the two diseases. There is no doubt that the two diseases are quite distinct in etiology even though rare cases of infection of birds with Bacterium diphtherio have been reported.

SIMPLE CATARRH OR COLD

This condition is a mild inflammation of the nasal passages and is common to all species of domesticated birds. It cannot be differentiated from the early stage of avian diphtheria.

Etiology. No definite cause may be assigned to this affection. It has been asserted that weak or improperly nourished birds are more likely to be attacked than strong, vigorous, well nourished individuals. Exposure to unfavorable conditions in which rain or dampness aceompanied by cold draughts tend to so affect the mucous membranes of the nostrils that the various organisms which are normally present in this region become pathogenic, has been held to be the primary contributing cause.

Symptoms. The affected fowl is more or less dull in appearance, according to the severity of the attack. The appetite is diminished, breathing becomes difficult, and a watery discharge from one or both nostrils is early in evidence. This discharge may disappear in the 
course of two to four days, or it may take on a viscid consisteney, closing the nitsal openings and necessitating breathing through the mouth. The discharge may also eseape through the mouth. This is rendered easy by the cleft palate peculiar to birds.

Morbid anatomy. The mucous membrane of the natsal prasiages becomes swollen and congested and an exeessive secretion of mucous fluid is poured out.

Treatment. As the disease is of short duration, it is usually only necessary to place the patient under more favorable conditions to bring about recovery. In the more severe cases, the nostrils should be washed out twice daily with boracic acid in 3 per cent solution, or creolin in 1 per cent solution.

\section{REFERENCES}

1. Beach. Suggestions to poultrymen concerning chicken pox. Univ. of Calif. Coll. of Agr. Circ. No. 145, 1915.

2. Beach, Lothe and Malpin. An outbreak of roup and chicken-pox in which the high mortality was apparently caused by a secondary invader. J. Agr. Research, Vol. 17, 1915, p. 554.

3. Bertegh. Ueber die Beziehungen zwischen Geflügeldiphtherie und Gefliigelpocken. Centralbl. f. Bakteriol. (Etc.), 1. Abt., Orig., Bd. 67, 1912, S. 43.

4. Bordet and Fally. Le microbe de la diphtherie des poules. Ann. de l’ Inst. Pasteur, T. 24, 1910, p. 563.

5. Burnet. Contribution a l'epithelioma contagieux des oiseaux. Ann. de l'. Inst. Pasteur, T. 20, 1906, p. 742.

6. Gallagher. Epithelioma contagiosum of quail. J. Am. Vet. Med. Ass., Vol. 3, 1916, p. 366.

7. Hadley and Beach. Controlling chicken-pox, sore-head or contagious epithelioma by vaccination. Proc. Am. Vet. Med. Ass., 1913, p. 704 .

8. Halasi. Beitrage zur Kenntniss de Geflugelpocke und der Geflugel diphtherie. Inaug. Diss. Közl.

9. Haring and Kofoid. Observations concerning the pathology of roup and chicken pox. Proc. Am. Vet. Med. Ass., 1911, p. 413.

10. Harrison and Streit. Roup. Ontario Agr. College. Bulls. 125 and 132.

11. Hausser. Bacteriologische Untersuchungen über Geflugeldiphtherie. Centralbl. f. Balteriol. (Etc.), 1 Abt. Orig., Bd. 48, 1909, S. 535.

12. Jackley. A study of the etiology of roup in birds. Kansas Agr. Exp. Sta. Tech. Bull. 4, 1917.

13. Mack. The etiology and morbid anatomy of diphtheria in chickens. Am. Vet. Rev., Vol. 28, 1905, p. 919.

14. Mack and Records. The control of epithelioma in chickens by raccination. Nevada Agr. Exp. Sta. Bulls. 82 and 84.

15. Manteufel. Beiträge zur Kenntniss der Immunitätserscheinungen hni den sngennannten Geflügelpocken. Arb.a.d.k. Gsndhtsamte., Bd. 33, 1909-10, S. 305. 
16. Marx u. Sticker. Untersuchungen über das Epithelioma contagiosum des Geflügels. Deutsche Med. Woch., Bd. 28, 1902, S. 893.

17. Moore. A preliminary investigation of diphtheria in fowls. $U . S$. Dep. Agr. Bureau Animal Indust., Bull. No. 8, 1904.

18. Müller. Zur Aetiologie der Geflügeldiphtherie. Centralbl. f. Bakteriol. (Etc.) 1 Abt. Orig., Bd. 41, 1906, S. 423.

19. Reischauer. Uber die Pocken der Vögel, ihre Beziehungen zu den echten Pocken und ihren Erreger. Centralbl. f. Bakteriol. (Etc.), 1 Abt. Orig., Bd. 40, S. 356.

20. Schmid. Untersuchungen über der Beziehungen zwischen Geflügeldiphtherie und Epithelioma contagiosum. Centralbl. f. Bakteriol. (Etc.), 1 Abt. Orig., Bd. 52, 1909, S. 200.

21. Sigwart. Experimentelle Beiträge zur Frage der Identität von Geflügeldiphtherie und Geflïgepocken. Cenlralbl. f. Bakteriol. (Etc.), 1 Abt. Orig., Bd. 56, 1910, S. 428.

22. Sweet. A study of epithelioma contagiosum of the common fowl. Univ. of Calif. Pubs. in Zoology, Vol. 71, 1913, p. 29.

23. Uhlenhuth und Manteufel. Neue Untersuchungen ïber die ätiologischen Beziehungen zwischen Gefiügeldiphtherie (Diphtheria avium) und Gefligelpocken (Epithelioma contagiosum). Arb. a. $d . k$. Gsndhtsamte, Bd. 23, 1909-10, S. 288.

24. Ward. Poultry diseases in California. Proc. Am. Vet. Med. Ass., 1904, p. 164.

25. Ward. Observations on roup in chickens. Proc. Am. Vet. Med. Ass., 1905, p. 198. 


\section{CHAPTER X \\ ASPERGILLOSIS AND FAVUS}

\section{ASPERGILLOSIS}

Synonyms. Pneumo-mycosis, brooder pneumonia.

Characterization. Aspergillosis is a rlisease of the pulmonary region due to infection with fungi. It is characterized by the formation of an exudate, usually of a moldy appearance, on the mucous membrane of the air passages, principally the air sacs of the abdominal cavity.

Etiology. The disease is almost invariably introduced hy fungi of the genus Aspergillus, Aspergillus fumigatus being the most constant causative agent. Other members of the aspergillus group have been identified in the morbid processes. These, however, are of slight significance as they have not been associated with the incidence of pulmonary mycosis to any great extent.

Aspergithes fumigatus is widely distributed in nature and is often observed on vegetable matter of all kinds which has been exposed to dampness. Birds scratching among moldy grains or in moldy litter may inhale the spores. These find a favorable place for propagation on the mucosa of the bronchi, or of the air sacs.

The fungus may be readily grown on potato, rye bread or plain agar which is acid in reaction. The most suitable temperature is between $35^{\circ}$ and $40^{\circ} \mathrm{C}$. The growth appears after 24 . hours incubation as a whitish downy layer over the surface of the medium. This is composed of an interwoven mass of trans-

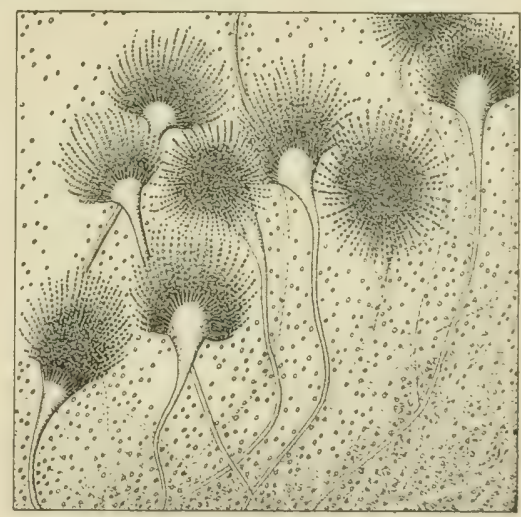

FIG. 10. Aspergillus fumigatus. (Redrawn from Mohler and Buckley)

parent mycelial threads which later give off branches culped ly truit heads. Each of the latter is composed of a mass of small rounded 
bodies closely associated in the form of a ball, the outer area of which shows radiating lines of spores. These are clear and highly refractile. Their diameter varies from 2.5 to 3.5 microns. The color of the growth changes with age, succeeding from white to greenish and later, in old cultures, to a brownish color. The spores are quite resistant. They are not destroyed by heating at $65^{\circ} \mathrm{C}$. for a period of seven hours and require an exposure of 12 hours to a 5 per cent solution of carbolic acid for their destruction. In the dried state, they are very resistant and may germinate after several months or even several years when conditions are favorable.

Fathogenicity. Of the aspergillus group, A. fumigatus is the most pathogenic to birds. A. nigressens, A. glaucus and A. candidus are less pathogenic in the order named.

All species of birds are apparently susceptible to infection. Domesticated ones, especially those confined in large flocks and exposed to moldy organic matter, are most often affected. These include chickens and pigeons. Young chicks frequently are attacked by an acute form known as brooder pneumonia. Cage birds, particularly those in zoological gardens, are often fatally affected. In France thousands of pigeons are fed by men in a manner similar to the method used by parent pigeons in feeding their young; viz., loading the mouth with grain and fluid and passing it directly to the mouth of the pigeon. Among these men severe cases of pulmonary aspergillosis occur as a result of their exposure to contaminated grain.

The disease may be transmitted artificially to animals and birds by inoculation or by causing the organism to be inhaled. Inoculation of small quantities of spores into the veins or peritoneal cavity of rabbits, guinea pigs, fowls, or pigeons causes death from septicemia in from 24 hours to 1 or 2 weeks. In the older cases necrotic areas are present in the viseeral organs and the aspergillus fungus is readily isolated from these. Lesions resulting from inhalation infection are usually confined to the air passages. Fowls and animals exposed in rooms to dust or rye flour containing aspergillus spores, have contracted the disease and died as a result.

Aspergillosis in the ostrich is discussed on pp. 228 and 229.

Mortality. Fatalities may be high in broods of chicks that have been extensively exposed to infection. Those attacked apparently all succumb. Older birds are quite resistant. Evidence points to the conclusion that once the disease is established in the system a fatal termination follows.

Symptoms. Considerable variation in the external signs of as- 
pergillosis may be expected. As a rule the morbid condition has existed for some time before symptoms are noticed. When the lesions are located in the trachea or loronchi, hoarseness of breathing, or a rattle in the throat may be the only indication of infection in the early stages. In air sac involvement alone no respiratory symptoms are shown. It is doubtful if external symptoms of infection of the air channels of the bones of the wing are manifested.

As the disease progresses the bird becomes dull and less active. There is a noticeable decrease in weight acempanied by emaciation. The term "going light" is frequently applied to this condition. Diarrhea is not a constant symptom but is usually present in the later stages. Temperature elevation is not constant in the more chronic form. Difficult respiration, sleepiness, extreme weakness and marked emaciation precede death. The appetite decreases as the disease progresses. Death results from asphyxia and toxemia.

In baby chicks the symptoms are apparent early and are similar to those of bacillary white diarrhea and intestinal coccidiosis. In both young and old birds the lesions observed at post mortem readily reveal the nature of the disease.

Morbid anatomy. The lesions are principally confined to the respiratory system. This system is complicated in birds for in addition to the lungs it includes several air sacs in the peritoneal cavity and air channels in certain of the bones. In some instances through penetration of the walls of blood vessels by the mycelia, new areas of infection may be established in other organs as a result of metastasis.

The mold may develop at any point on the respiratory mucous membrane or may be spread over a large portion of its surface. Our observations at numerous autopsies place the abdominal air sacs as the most severely involved region. The morbid process is manifested by the presence of a membrane of greater or less thickness depending on its location on the mucosa, or at times by nodules in the parenchyma of the lungs. In the trachea and bronchi the false membrane is comparatively thin while in the air sacs it may be much thicker and give a rigid contour to these organs. Frequently the sacs are filled with fungoid growth and cellular exudate, the whole forming a solid mass. The bronchioles may also be pluged with mycelia, spores and cellular exudate consisting of exfoliated epithelial cells, lenecertes and oceasionally red blood compuseles. The surface of the membrane may be grayish or greenish in color. The latter color is nearly always in evidence at some point. It is in 
this area that numerous spores may be demonstrated on microscopic examination.

The mycelial threads penetrate the mucosa to the submucosa or may enter the adjacent blood vessels forming thrombi and establishing an area of necrosis resulting in a nodular formation similar to that of tuberculosis. In the abdominal region the organs in contact with the air sacs are often involved through adhesions or direct passage of the fungus into their interior.

In the main, the pathologic condition progresses by direct contact. Organs affected through the blood stream reveal small areas of necrosis in which the mycelial threads may be detected. The kidneys are more often affected in this manner than the liver. Artificial inoculation through hypodermic injection, intraperitoneally or intravenously, produces extensive lesions in these organs.

The mycelia may be demonstrated microscopically in necrotic areas or in nodules in the earlier stages of development. Mycelia may also be observed in the mucous membrane lesions, and especially in greenish colored areas are spores readily discovered. The tissues surrounding the growths are found infiltrated with small round cells, leucocytes and connective tissue cells, indicating an attempt on the part of the system to build a protective wall against the spread of the parasite. This attempt is usually abortive although evidence of regenerative changes are apparent in some lesions. Giant cells are also occasionally present in the tubercle formations.

Course of disease. Among older birds the disease has a tendency to occur sporadically, more or less extensive intervals elapsing between deaths from this cause in a particular flock. Where the exposure is great, outbreaks of considerable importance may result. The resistance of vigorous individuals, however, is high and epizootics such as are associated with certain other diseases are rarely encountered. In grown birds aspergillosis follows a semi-acute or chronic course. Death may occur in from 1 to 8 weeks. In brooder chicks resistance is less pronounced and acute outbreaks often follow exposure to moldy food or material. The disease runs a rapid course with fatal termination in 2 to 7 days. Birds confined to limited areas as is the case in zoological gardens, show a high percentage of fatalities from this cause.

Diagnosis. Tuberculosis, coccidiosis and aspergillosis are not readily differentiated symptomatically in grown fowls. On autopsy the lesions are quite distinctive. Tuberculosis principally affects the 
liver, intestine and spleen, being less of a pulmonary disease in birds than is the case in other animals. The tubercles are of a fleshy type on cross section and show small yellowish points. Coccidiosis is practically confined to the intestinal tract. Its lesions are of a necrotic type involving principally the duodenum and ceca. Aspergillosis tends to localize on the walls of the air passages and forms a moldy growth generally exhibiting a greenish tinge, at least in spots.

Microscopic examination of the morbid material furnishes a specific diagnosis since the causative organisms in each case are usually numerous and very different in morphology.

The disease in young chicks called brooder pneumonia, shows symptoms similar to those of bacillary white diarrhea and coccidiosis. In all three the chicks have a droopy appearance associated with diarrhea and loss of appetite. In general it may be said that bacillary white diarrhea is more highly fatal, with deaths oceurring shortly after hatching. Coceidiosis usually appears at a later period and is also apt to result in heavy losses. Autopsy is necessary, however, for positive diagnosis. The lesions in the air passages are not as extensive as in older fowls because of the acuteness of the affection, but may be discerned by careful examination. Bacillary white diarrhea is a purely septicemic disease, while coccidiosis usually shows intestinal lesions with accumulation of necrotic or blood stained material in the ceca. Microscopic or cultural methods may be resorted to in obscure cases.

Treatment. It is inadvisable to attempt treatment because of the deep seated position of the parasitic fungus, its resistance to remedial agents and the advanced stage of the attack when srmptoms are observed.

Prevention. The widespread distribution of Aspergillus fumigatus renders absolute prevention difficult. Care in the selection of grain and litter will minimize to a great degree the chances of infection. Good housing arrangements which guard against dampness will prevent the propagation of molds if present to a slight extent in feed or litter. When the disease is discovered in a flock the source of infection should be located. The feed may have a musty odor or a greenish appearance in places, or straw litter may be moldy. In the former instance cooking will destroy the funguis. Moldy litter should be discarded.

Experiments have shown the possibility of infection being carried to newly hatched chicks through the egg. The mycelia of the fungus 
are known to be capable of penetrating the egg shell and developing in the albuminous material with consequent destruction of the embryo. The aspergillus colony may be observed as a dark spot on the internal membrane of the air chamber. Infection through this source is no doubt rare. It may be guarded against by using clean straw or chaff in nests for laying or sitting hens, and by dipping eggs for hatching in grain or wood alcohol before incubating. Feeding discarded eggs to young chicks is dangerous since these may carry the causative organisms of several diseases.

FAVUS

Synonyms. White comb; favus de la poule (French); Weisser Kamm (German).

Characterization. Farus is a contagious, chronic disease of the skin characterized by the presence of white areas most commonly localized on the head and is caused by a fungus.

Etiology. The fungus causing the disease is designated Lophophyton gallince. It grows readily on agar containing 1 per cent peptone and 4 per cent of glucose or maltose at room temperature when the medium is seeded with material from the diseased patehes. Growth first appears as a small, round, pure white, downy colony. As it enlarges it takes the form of a button which is slightly cup shaped. The culture ordinarily remains white if kept at a low temperature. At $27^{\circ}$ to $37^{\circ} \mathrm{C}$. the colony takes on a delicate rose color, becomes umbilicated, wrinkled and divided into sectors by lines radiating from the center. At $30^{\circ} \mathrm{C}$. the culture is wholly rose colored, but more or less deep depending upon the culture. Sometimes the color varies in the same culture tube. The diffusion of the raspberry color in the medium is said to be an exclusive character of the species in question.

Pathogenicity. The infection is readily transmitted to a fowl by rubbing the comb with scales from a diseased bird. The discase is unmistakably present on the fifteenth day after inoculation and persists for months. Similar results are obtained with cultures. Mice, rabbits, guinea pigs and man are susceptible. Infection may oceur through direct or indirect contact.

Symptoms and morbid anatomy. The disease first appears on the head and its appendages such as the comb, wattles and barbs. Nearly always the initial lesion is in the vicinity of the beak, in the form of numerous small white points. Under a magnifying glass 
they are seen to be covered with a fine, short down which later disappears quickly and does not return again. The white point enlarges and forms a layer 1 or $2 \mathrm{~mm}$. thick adhering to the epidermis underneath. The color is generally white or gray. In time the thickness of the crusts increases as do the surface dimensions. Finally the small patches originally isolated, coalesce. The extension is regular. A white patch may reach the dimensions of the comb itself. Sometimes the dimensions of a patch remain small but it is not rare to observe patches 7 or $8 \mathrm{~cm}$. long and 3 or $4 \mathrm{~cm}$. broad on large combs.

The diseased area examined closely presents for study thin places and thick places. Those which form small hard eminences of a grayish white color or slightly reddened at the top, are irregularly disseminated over the area of the plaque. Between these

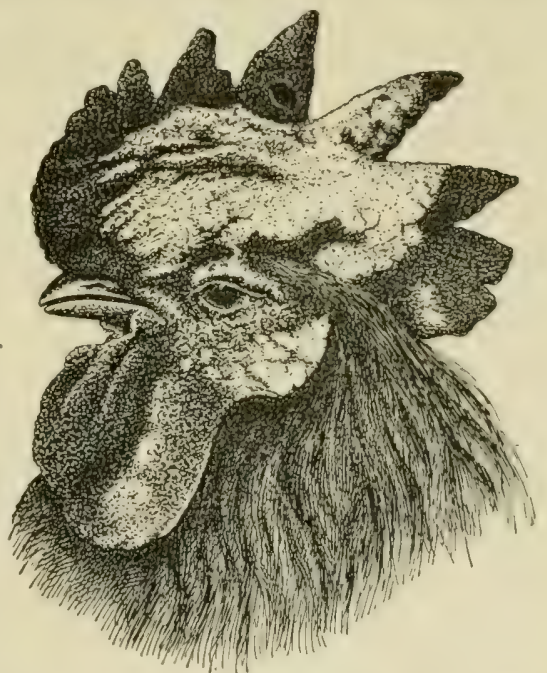

FIg. 11. Favus, involving the head. (Drawn from photograph by Sabouraud, Suis and Suffran)

elevations the patch is merely a thin membrane of a pearly white color. Wherever the white area is thickened, the scratching detaches small fragments like a white powder, of which certain pieces resemble mica flakes.

When the lesion in extending, encounters the feathers, its appearance changes slightly. A deposit of whitish crusts accumulates at the base of each feather. Some feathers fall out spontaneously, and all those which have this squamous collar at the base, have little resistance against being plucked. If a feather is pulled it comes out entire with its yellow root part, but it bears with it, like a collar, the whitish deposit which surrounds the point where the feather emerges from the skin.

Course of disease. The disease is benign. Instances in which affected birds become cachectic and die, are exceedingly rare. Recovery may oceur in three months spontanenusly, or the disease may last for years. Long duration may be considered as an example of a succession of infections, rather than the persistence of one infection. The disease persists in subjects associating with other infected 


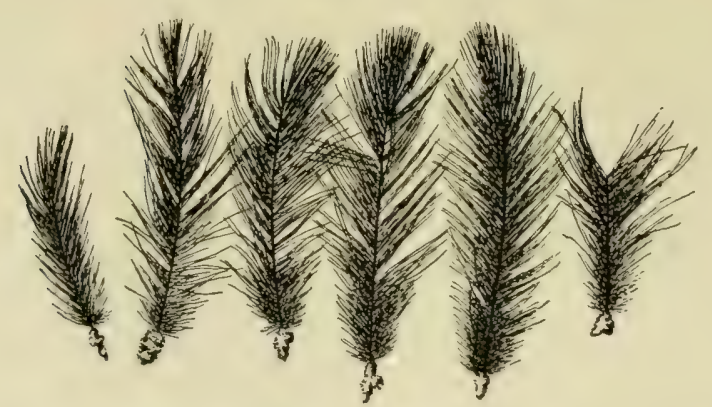

Fig. 12. Deposit of crusts on quills of feathers occurring in favus involving feathered areas. (Drawn from photograph by Sabouraud, Suis and Suffran)

birds, in infected quarters, while there is a tendency to recovery when affected birds are isolated.

Treatment. Isolation of affected birds from the flock and from other infected individuals in some cases is sufficient to cause the disappearance of the disease. Previous to the application of any remedy the affected areas should be softened with soap and warm water, after which as much of the deposit as possible should be removed. The comb lesions may be treated with carbolized vaseline or green soap containing five per cent of carbolic acid. A mixture of glycerine 6 parts and iodine 1 part has been employed on the comb as have salicylic acid ointment $(1: 10)$; tincture of iodine diluted with equal parts or more of alcohol; five per cent formalin ointment or solution; and an ointment made of red oxide of mercury 1 part, to 8 parts of lard. A $1-500$ solution of bichloride of mercury may be used among the feathers.

Prophylaxis. In an infected flock, the closest examination of the exposed birds should be made, so that those slightly infected may be isolated and treated. Otherwise they will perpetuate the infection in the flock. Care should be exercised to prevent the introduction of birds from flocks not positively known to be free from the disease. In an infected flock, thorough cleaning and disinfection of the quarters should be carried out.

\section{REFERENCES}

1. Balfour. Aspergillary pneumokoniosis in the lung of a turkey. Fourth Rep. Wellcome Research Lab., 1911.

2. Beach and Halnin. Observations on an outbreak of favus. J. Agric. Research, Vol 15, 1918, p. 415. 
3. Lignières et Petit. Péritonite aspergillaire des dindon. Rec. de Méd. Vét., T. 75, 1898, p. 145.

4. Lucet De l' Aspergillus fumigatus chez animaux domestiques et dans les oefs en incubation. Paris, 1897.

5. Matruchot et Dassonville. Recherches expérimentales sur une dermatomycose des poules et sur son parasite. Rev. Gén. de Bot., T. XI, 1899, p. 430 .

6. Mohler and Buckley. Pulmonary mycosis of birds. U. S. Dep. Agr. Bureau Animal Indust. Circ. 58, 1904.

7. Neumann. Aspergillosis in domesticated birds. J. Comp. Path. and Therap., Vol. 21, 1908, p. 260.

8. Sabouraud. Le trichophyton de la poule et la maladie humaine qu'il détermine. Arch. de Méd. Expérim., Mai 1909.

9. Sabouraud, Suis et Suffran. La "crête blanche" (favus) de la poule et son parasite. Rev. Vét., T. 34, 1909, p. 601. 


\section{CHAPTER XI \\ INFECTIOUS ENTERO-HEPATITIS OF TURKEYS}

\section{Synonym. Blackhead.}

Characterization. Entero-hepatitis is an infections disease of turkeys and fowls, characterized by distention of the ceca with necrotic material and the formation of yellowish or yellowish-green necrotic areas in the liver.

Geographic distribution. It is widely distributed over the United States, and has also been reported from Canada, Europe, Australia and Africa.

Etiology. The causative organism as deseribed by Snith belongs to the genus ameba and has been designated by him Ameba meleagridis. Through the researches of Hadley, the rôle of an ameba in the causation is disputed. The latter investigator asserts that a flagellated protozoal organism, Trichomonas, is the causative agent and that the organism described by Smith merely represents a transitory stage in the life cycle of the flagellate. The difficulties to be met with in establishing the etiologic factor of this disease are far greater than is the case in bacterial affections. In routine examination of entero-hepatitis cases by the present writers, it has been observed that flagellate forms are frequently present in the cecal exuclate of turkeys dead of entero-hepatitis while more often protozoal organisms of the ameba type are seen in comparatively large numbers. Further and more extensive investigation appears to be necessary in order to establish firmly the specific etiology of this disease.

The organism described by Smith is round or oval in form with a single contoured outline. The structure appears homogeneous throughout with the exception of a small granular area eccentrically located and representing the nucleus. This encloses a smaller rounded body distinguished as the nucleolus. The protozon in fixed or hardened tissue are from 6 to 10 microns in diameter, in fresh smears from 8 to 15 microns in diameter.

The organism attributed by Hadley as the causative agent is a flagellate. This is most easily recognized in the motile stage. In this form it presents an irregular outline. It may be pear shaped, ovoid, triangular or elliptical in form. It has three anterior fla- 
gella, a vibratory membrane and one posterior flagellum. The nucleus is placed anteriorly. One or more food racuoles may be observed. The average length of this form is about 10 microns.

Pathogenicity. Young turkeys are especially suseeptible, although there appears to be no period of immunity to primary infection during the life of the bird. The greatest losses vecur during the first two or three months of life. Practically all of the exposed poults contract the disease at this time. Young chickens are only slightly susceptible but may serve as carriers of the organism.

Source of infection. The disease is principally spread throngh exposure to infested ground. Once the causative agent has been brought on the premises, it is apt to continue its activity year after year. Whether this is wholly due to the resistance of the encysted stage of the parasite, to climatic or other external influences or whether it is perpetuated in the intestinal tract of comparatively resistant turkeys or chickens is open to speculation. From the fact that mature turkeys may show symptoms of entero-hepatitis during all seasons of the year, it is reasonable to assume that they constantly harbor the organism in a saprophytic form and only develop the disease as a result of adverse conditions or weakened constitution from other causes. Curtice states that chickens though rarely infected may serve as carriers. In any case, the disease is contracted by the poults ranging on ground infested by the droppings of carriers of the parasite. The parasite passes from the intestine in the encysted or resistant stage. In this form when not exposed to the direct rays of the sun or to considerable drying it may retain its vitality for a long period, possibly one to two years. Earth worms may play some part as mechanical carriers of eysts which have survived in the soil under favorable conditions. When these encysted forms are taken into the alimentary tract by poults in feeding, they undergo changes which renew their activity. The tough resistant cuvering is discarded and forms developed from the central granular mass or nucleus penetrate the mucosa of the intestine, especially the ceca and may be carried by the blood stream to the liver. The parasites multiply rapidly in the intestine and are passed out in enormous numbers in the droppings. By this means, other susceptible birds in the flock are directly infected through coming in contact with the contaminated material in feeding.

Symptoms. The srmptoms of entero-hepatitis become apparent within a period of four weeks after exposure. Toung birds show less resistance and develop manifestations earlier than grown ones. 
The disease runs a rapid course in the former and deaths may follow in a few days. Older turkeys, fatally affected, usually succumb within four weeks after the onset of symptoms. The mortality approaches 90 per cent in poults and 10 per cent or more in the grown birds, according to the extent of exposure. Affected poults exhibit a drowsy attitude with constantly increasing lack of vigor. They are unable to keep up with the flock in its daily travel over large areas. The appetite is diminished, the feathers ruffled, wings are pendant, and a general air of debility is manifested. Diarrhea is nearly always present and quite characteristic. The droppings are soft and yellowish in color or may be mixed with brownish excrement. During the later stages the head may present a darkened or purple appearance due to impaired circulation of the blood. This symptom has led to the disease being termed "blackhead." However, this term is misleading since the darkened appearance of the head is not a constant symptom and may also be observed in other diseases which lead to a weakened heart action. In older turkeys the symptoms are less pronounced and may be of much longer duration. They show loss of appetite, unthrifty appearance, lessened activity, progressive emaciation and diarrhea, with soft yellowish droppings. The disease may persist for a period of two weeks to two months and result fatally or the bird may regain its normal condition. Relapses frequently oceur during the following year. The disease is more prevalent during the late summer or fall in young poults but may occur at any season and is frequently reported among grown turkeys during the winter and spring.

Morbid anatomy. The lesions of entero-hepatitis are confined to the intestine and liver. In the intestinal tract the ceca are the principal seat of infection. In many cases only one cecum exhibits macroscopic alterations but quite frequently both ceca are equally involved. The walls become greatly thickened and covered by a neerotic exudate which completely fills the cavity or leaves but a small central space. The accumulation of exudate gives rise to a marked distention of these blind pouches plainly apparent when the intestine is exposed at autopsy. The exudate is dull gray in color and of a friable or cheesy consistency. The entire length of the cecum may be involved or only a limited area, depending on the severity or duration of the morbid process. In the majority of cases the liver is found infected to a greater or less degree. The lesions are characteristic. Areas of degeneration appear on the surface in circular form and of varying size. They are usually about 
$1 / s$ to $\%$ of an inch in diameter and may be isolated and few in number or numerous and closely associated, even coalescent. Their color varies from a yellowish to a yellowish-green. These areas of necrosis extend more or less deeply into the structure of the liver and result from plugging of the smaller arterioles with the parasites and cellular debris. They are not always of a homogeneons color, but may appear mottled with small areas of approximately normal liver tissue. No elevation above the liver surface is in evidence as may be the case in tuberculosis or sareomatosis of the liver and the tendency is rather to a slight depression beneath the capsule. The liver is often enlarged to a considerable degree and congested.

In very acute cases the ceea show slight evidence of exudate but are more or less inflamed while the liver is free of lesions. Other areas of the intestine, especially the duodenum and rectum, harbor the causative parasite and may exhibit congestion of the mucous membrane, hemorrhagic points or even spots of necrosis. However, the ceca and liver are the main seats of disease and more to be relied upon for diagnostic evidence. Infrequently the morbid process within the lumen of the cecum extends through the mucosa, submucosa, muscular layers and serous coat causing perforation and exudation into the peritoneal cavity leading to peritonitis or extensive adhesions of the cecum to the surrounding loops of intestine. This condition is apparently due to the action of invading bacteria since the protozoal organisms have not been demonstrated in these secondary lesions.

Microscopically the cecal exudate is found to be composed of protozoal bodies, exfoliated mucosa cells in various stages of degeneration, connective tissue shreds, blood cells and an amorphous detritus. The cecal wall is greatly thickened due to infiltration and connective tissue hypertrophy. The mucosa shows extensive degenerative changes or is absent in areas. Depending on the depth of the ulceration, the circular or longitudinal muscular layers show more or less infiltration with small round cells and congestion, which may extend to the serosa. In the liver the necrotic foci show varying stages of cell degeneration and are surrounded by a congested area. Walling off of the affected spots is not well marked or is totally absent. Small round cells, protozoal organisms and nuclear remnants are distinguished in the amorphous necrotic material. In both the intestinal membrane and in the liver the protozoa occur either singly or in groups between the epithelial, or parenchrmatous cells where they may be enclosed in a connective tissue network. 
They have also been observed in the interior of giant cells (Smith), and epithelial and endothelial cells (Hadley).

Diagnosis. Entero-hepatitis is readily differentiated from other diseases by the definite character of its internal manifestations. The presence of yellowish or yellowish-green spots on the surface of the liver is specific. Turkeys are much less frequently affected with tuberculosis, or malignant tumors than fowls and these diseases may be eliminated where several birds in a flock become infected at the same time, especially in the case of poults. The lesions of tuberculosis, on cut section, present a fleshy appearance whitish in color with yellowish points and usually extend to the spleen, intestine and mesentery where they appear as rounded nodules. Tumor formations vary greatly in size, are fleshy in character and are usually found on the serous membrane. They are of a chronic character and oceur sporadically. In tuberculous infection, tubercle bacilli will be found in large numbers on microscopic examination of smears from the nodules.

Treatment. Extensive experiments looking to the treatment of entero-hepatitis have so far failed to develop a satisfactory method. The insidious nature of the affection and the deep seated location of its parasite in the wall of the intestine and in the liver render it practically immune from interference by curative agents. Emetin hydrochlorid, a derivative of ipecae, by repeated hyporlermic injection has given more or less satisfactory results in the treatment of amebic dysentery in man, a disease somewhat analogous to entero-hepatitis of turkeys. However, the above disease of man is of a chronic nature and lends itself to a more prolonged course of treatment than is permitted in the case of the comparatively acute disease under consideration. Also the value of young turkeys does not justify the time and expense which would be involved even were the treatment fairly successful. Giving ipecac in small doses might prove beneficial. The most promising and economical treatment is the administration of crude catechu by means of the drinking water. This has been recommended by Fantham for cocidiosis of fowls and has given good results in our experience. One-third teaspoonful of crushed crude catechu is added to each gallon of water. It is quite possible that the use of catechu in this manner during the first three months would bring the exposed poults safely through the most dangerous period. Should signs of constipation develop as a result of this treatment, the entire flock may be given Epsom salts in the proportion of one teaspoonful to every ten poults. The salts can 
be dissolved in water and the solution mixerl with a feed to be given early in the morning: Turkeys on range would require special attention for the successful operation of the above measures. Isolation or disposal of birds showing symptoms of disease is of the first importance in guarding against a further spread of the malady. Thorough cleaning and disinfection of quarters and yards will tend to keep the outbreaks in cheek. Carbolic acid in 5 per cent solution, or compound cresol in 3 per cent solution are efficient disinfectants.

Prevention. Prevention of entero-hepatitis in infected communities is more difficult than the prevention of other diseases of domesticated birds, because of the fact that turkeys range over an area of several miles in diameter when allowed to run at large as is the usual custom in this country. The possibility of healthy flocks becoming infected by feeding on ground contaminated by diseased flocks is very apparent and renders preventive measures under such conditions rather difficult of execution. Where turkeys are confined to a limited area or do not come in contact with other flocks which harbor the parasite, simple precautionary measures may insure freedom from the disease. The main channel of entrance is through the introduction of birds which come from infected flocks. Particular care in selecting purchased birds should be exercised. The fact should be established beyond doubt that they are of a flock which has shown no symptoms of disease and have not been associated with neighboring diseased flocks. A period of quarantine extending over 30 days is also advisable before newly acquired turkeys are placed with the home flock.

Where the disease has been prevalent on premises to such an extent as to make the raising of turkeys unprofitable, it is advisable to dispose of the entire lot and allow a period of approximately two years to elapse before restocking. Local conditions may modify or suggest preventive measures. Limeing or plowing the runs or home feeding yards is indicated. The principal fact to be considered is that the disease must first be introduced, either by infected birds (almost invariably the turkey) or by mechanical carricrs, such as persons coming from infected farms and carrying the causative parasite on their shoes. Wild birds may possibly serve as carriers. Other agencies eould be mentioned by means of which the organism of entero-hepatitis might be earried from infected farms; howerer, these are of minor importance and cannot easily be guarded against. Eggs for hatching, from infected turkeys may be dipped in 95 per cent alcohol to destroy any parasites which may have become at- 
tached to the shell. Practically the only danger in using eggs from infected flocks is that the shells may have come in contact with droppings harboring the infectious parasite.

On first indication of the presence of entero-hepatitis the affected birds should be destroyed and their carcasses burned or deeply buried. The quarters should be thoroughly cleaned and disinfected with 5 per cent carbolic acid or compound cresol in 3 per cent solution. The runs or local feeding ground should be cleaned and limed or plowed under as the chance for infection is greatest at the points where the flock congregates most often. The confinement of turkeys to inclosed yards has been tried with apparently favorable results. By this method of rearing, entero-hepatitis could be more easily controlled.

Hadley regards the prevention of Trichomonas infection in turkeys as presenting a somewhat different problem from that involved in the prophylaxis of many diseases. The parasites are found normally present in the intestines of all poultry. Their pathogenic activity in turkeys, in his opinion, depends upon factors present in the host which are probably quite unrelated to virulence on the part of the infecting organism. This view would suggest the exercise of great care in feeding in order to preserve health even though the flagellates are present in the intestine.

\section{REFERENCES}

1. Curtice. Notes on experiments with blackhead of turkeys. U.S. Dep. Agr. Bureau Animal Indust. Circ. 119, 1907.

2. Curtice. The rearing and management of turkeys with special reference to the "blackhead" disease. Rhode Island Agr. Exp. Sta. Bull. 123.

3. Curtice. Further experiments in connection with the blackhead disease of turkeys. Rhode Island Agr. Exp. Sta. Bull. 124.

4. Cushman. Nature of blackhead in turkeys. Rep. Rhode Island Agr. Exp. Sta. 1894.

5. Hadley. Blackhead in turkeys. A study in avian coccidiosis. Rhode Island Agr. Exp. Sta. Bull. 141.

6. Hadley. The rôle of the flagellated protozoa in infective processes of the intestines and liver. Rhode Island Agr. Exp. Sta. Bull. 166.

7. Milks. A preliminary report on some diseases of chickens. Louisiana Agr. Exp. Sta. Bull. 108.

8. Moore. The direct transmission of infectious entero-hepatitis in turkeys. U. S. Dep. Agr. Bureau Animal Indust. Circ. 5, 1896.

9. Smith. Infectious entero-hepatitis in turkeys. U. S. Dep. Agr. Bureau Animal Indust. Bull. 8, 1895.

10. Smith. Further investigations into the etiology of the protozoan disease of turkeys known as blackhead, entero-hepatitis, typhlitis, etc. J. Med. Res., Vol. 33, 1915, p. 243. 


\section{CHAPTER XII}

\section{COCCIDIOSIS}

Characterization. Coccidiosis is primarily a disease of the intestinal tract. In young chicks it may also affect the liver and in geese, the causative organism may be localized in the kidneys. It is one of the greatest scourges of fowls, pigeons and turkeys.

Etiology. The organism causing coccidiosis is designated Eimeria (Coccidium) avium and belongs to a class of protozoan parasites known as sporozoa. The organism is included in the animal kingdom and reproduces by both sexual and asexual processes. The parasite was long known as Coccidium avium but owing to the rules of priority in zoological nomenclature, the familiar name of the genus Coccidium has been replaced by Eimeria.

In the oöcyst or resistant stage, the one most easily recognized on microscopic examination of the intestinal contents, the protozoan generally appears as an oval borly containing a central granular mass surrounded by a highly refractile zone with a double contoured border. The size varies from 25 microns to 35 microns in length and from 15 microns to 20 microns in breadth.

Life history of Eimeria avium. The life history has been admirably worked out by Fantham. There are two principal stages in the life cycle, a stage of asexual multiplication, termed schizogony, and a stage of sexual reproduction in which male and female elements unite to form resistant bodies for life outside the animal cells. In the asexual stage the newly formed parasites pass from one cell to another, and it is during this cycle that the great destruction of intestinal mucosa occurs.

(a) The young, growing parasite. The oöcyst reaches the duodenum of the bird through the medium of the food or water. Here the tough cyst wall is softened and four small oval bodies or spores are released. From each of these are given off two actively motile vermiform bodies with one end more pointed than the other, which vary from 7 to 10 microns in length. These are the primary infecting germs or sporozoites (fig. 13 A). The sporozoite passes into an epithelial cell lining the first portion of the intestine where it curls on itself (fig. 13 B), takes on a spherical outline (fig. 13 C) and be- 


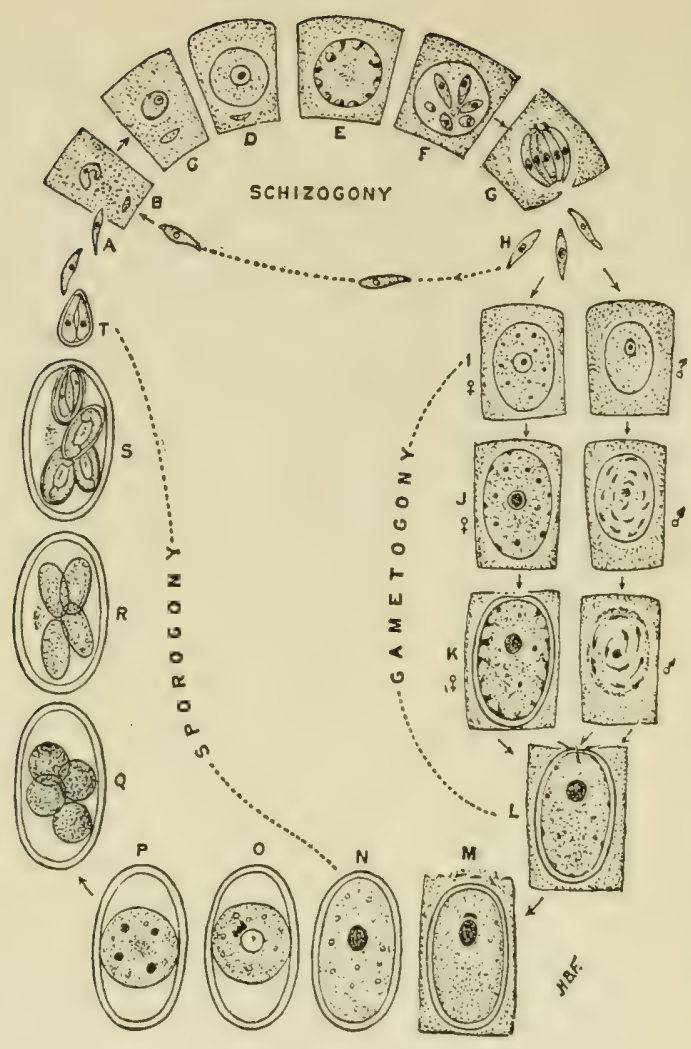

Iơ. Young microgametocyte with fine gramules.

Jf. Growing female mother cell, showing chromatoid and plastinoid granules. Jo". Microgrametocyte with nucleus divided to form a number of bent, rodlike portions, the future microgametes.

K\%. Macrogamete which has formed a cyst wall for itself but left a thin spot for the entry of the microgamete.

Ko". Microgametocste with many biflagellate microgametes about to separate from it.

L. Fertilization. One microgamete is shown penetrating the macrogamete, while other male cells are near the micropyle but will be excluded.

M. Fertilization. The male pronucleus is lying above the female chromatin. Degenerating microgametes are shown outside the oöcyst.

N. Oöcyst (encysted zygote) with contents filling it completely.

O. Oöcyst with contents concentrated, forming a central, spherical mass. Many such cysts seen in infected cecal droppings.

P. Oöcyst with four nuclei.

Q. Oöcyst with contents segmented to form four rounded sporoblasts. (As seen in fresh preparations.)

R. Oöcyst with four sporoblasts which have grown oval and are becoming sporocysts.

S. Oöcyst with four sporocysts, in each of which two sporozoites have differentiated.

T. Free sporocyst in which the sporozoites have assumed the most suitable position for emergence. 
gins to grow in size at the expense of the host cell. This is known as the trophozoite stage (fig. 13 D).

(b) Ascxual multiplication or schizogony. When fully dereloped the trophozoite has a diameter of 10 to 12 microns. In preparation for propagation the nucleus divides into a number of daughter nuclei which arrange themselves in a zone at the periphery. This constitutes the schizent stage and represents the begimning of asexual multiplication or sehizogony (fig. $18 \mathrm{E}$ ). Each nucleus becomes surrounded with protoplasm and assumes a long narrow form with pointed ends. The length ranges from 6 to 10 microns (fig. $13 \mathrm{~F} \mathrm{G}$ ). These bodies, known as merozoites, differ from sporozoites in that their nuclei contain a small particle of chromatin, the karrosome. From 8 to 20 merozoites are formed from 1 schizont. On separation from one another (fig. $13 \mathrm{H}$ ), the merozoites work their way into other cells, lining the intestinal tract and may repeat the process of development just outlined, or differentiation into sexual forms may take place within the newly invaded cells. This process is termed gametogony.

(c) Sexual reproduction or gametogony. Two forms of the organism are now produced, the macrogametocyte or female mother cell (fig. $13 \mathrm{I} \mathrm{J}$ ) and the microgametocyte or male mother cell (fig. $13 \mathrm{I} J$ ). Both forms are oval in shape, the former being somewhat larger and more granular than the latter. Also the former gives rise to only one daughter cell or macrogamete (fig. $13 \mathrm{~K}$ ) while the latter produces a large number of minute, biflagellate, actively motile male cells or microgametes (fig. $13 \mathrm{~K}$ ). The macrogametes are from 11.8 to 17.5 microns in length and 6 to 11 microns in breadth, in sections. The microgametes are 3 to 4 microns in length, in sections.

(d) Fertilization. Fertilization takes place through the entrance of the microgamete or male cell into the structure of the female cell or macrogamete at the thin spot at one end known as the micropyle (fig. $13 \mathrm{~L}$ ). After the entrance of the male cell the macrogamete secretes protoplasm which plugs the opening and prevents the entrance of other microgametes. On union of the nuclei of the two gametes, a zygote is formed which proceeds to spore formation or sporogony (fig. $13 \mathrm{M} \mathrm{N}$ ).

(e) Sporogony. The zygote is surrounded by a tough double contoured membrane which is extremely resistant. This stage is known as the egg stage, or oöcyst. The contents are at first granular and grayish in appearance (fig. $13 \mathrm{~N}$ ). Later the granular material is 
gathered into a compact circular mass approximately in the center of the oöcyst (fig. $13 \mathrm{O}$ ). The nucleus imbedded in the central matter now undergoes division into two and these in turn divide, forming four daughter nuclei (fig. $13 \mathrm{P}$ ). These are surrounded by cytoplasm and separated from each other. As a result four rounded bodies are formed within the cyst. These are sporoblasts (fig. $13 \mathrm{Q}$ ). Later the sporoblast loses its rounded form, becomes oval and is inclosed in a cell wall (fig. $13 \mathrm{R}$ ). In this form it is termed a sporocyst and from it develops a spore which contains two young infective parasites or sporozoites. The life cycle is completed by the rupture of the oöcyst wall in the intestinal tract of the host, which releases the spores and the primary infecting sporozoites.

From experiments made by feeding fowl chicks with coccidian oöevsts, Fantham concludes that the period for the total life cycle of the parasite is from eight to ten days.

Pathogenicity. Apparently coccidiosis is infectious to all domesticated and wild birds which are exposed to its causative organism. It has been observed in a wide variety of these birds and its incidence has been marked by high mortality, especially in those confined to limited areas. It is highly destructive to young chickens and frequently affects baby chicks. Its greatest ravages are seen in chickens which have passed the brooder stage and are exposed to the infective agent in infected houses or grounds. Limited range, as is enstomary in the rearing of domesticated birds, and especially in the case of chickens closely confined in large flocks, is conducive to heavy infection and high mortality in the event that coccidia are present. Next to chickens, pigeons are no doubt most severely affected. Outbreaks among ducks and geese occur less often as an epizootic unless the birds are closely confined, but individual infection commonly occurs.

Birds of all ages are susceptible. Mature birds exhibit a fair degree of resistance. They may harbor the coccidia and appear normal until they become grossly infected, or beeme weakened through other causes to such an extent that their power of resistance is lost.

Mortality. In chicks affected at the age of two to ten weeks, the disease runs a rapid course and the mortality may reach 100 per cent in closely confined flocks. In older fowls, the fatalities, while not so great, are usually very heavy in those surrounded by unsanitary conditions. Mature hens have a greater power of resistance but even among these, severe losses may be observed. The mortality is governed by the degree of exposure. 
Course of the disease. The coccidium when once introduced inio a flock develops rapidly in the cells of the intestinal mucosa, giving rise to a great number of sexual and asexual forms which are passed out in the droppings and are ingested by healthy birds. Accumulation of infected droppings naturally tends to increase the number of these organisms ingested and results in gross infection. Where fairly good sanitary conditions are maintained, the spreal of the disease is less general and the amount of infectious material consumed is proportionally lessened. As a result, birds which have a degree of normal resistance are protected against heavy infection.

Symptoms. The outward manifestations of coccidiosis depend to a considerable degree upon the age of the birds attacked. In young chicks, the disease develops rapidly and the mortality is high. The affected ones display the usual appearance associated with debilitating internal disorders, such as weakness, disordered feathers, droopy wings, bunched appearance, loss of appetite, and somnolence. The droppings are semi-fluid and usually whitish in color, but may be of a fluid nature with brownish tinge. In very acute cases in young chicks the droppings are deeply stained with blood. Affected chicks of an age of three weeks or less to two months seldom survive in a severe outbreak and those that recover are stunted and worthless. Chicks affected fatally succumb in from one to several days after outward symptoms are apparent, depending upon their age. The older ones show a stronger resistance, but in these too, the fatalities are extremely heavy.

In grown fowls, the disease usually assumes a more chronic type, although acute outbreaks are not infrequent. In these acute outbreaks, the affected birds show the droopy appearance which is more or less characteristic of all infectious diseases of fowls, and which, in itself, has comparatively little value as a diagnostic feature. In semi-acute cases, the fowl develops a progressively increasing listlessness and loss of activity. The comb becomes pale and periods of dejection, during which the bird stands in an isolated position, increase as the disease progresses. The appetite may remain normal and is frequently ravenous when the fowl is aroused at feeding time. During intervals between regular feeding, there is little effort to scratch for food with the others of the flock. The symptoms may he apparent for from one to three weeks, death occurring quite suddenly or after a short period of coma. In the more chronic form, the fowl retains a pale appearance of the comb and wattles for several weeks, the appetite appears normal while the bird is eating, 
but the amount of food consumed is less than usual, owing to the general indifference to food during prolonged intervals. Emaciation is marked both in semi-acute and chronic cases, principally due to the fact that the destruction of the lining membrane of portions of the intestine prevents normal assimilation of the food. Leg weakness and paralysis are frequently observed.

Affected geese become greatly weakened and emaciated. After walking a few steps they will fall and after a struggle roll on their backs, a position which is frequently assumed.

Morbid anatomy. The lesions are prineipally confined to the intestinal tract and are most noticeable in the ceca, in chickens and turkeys. In young chicks in which the disease assumes an acute course, the ceca are filled with a bloody semi-solid mass which shows through the intact tubes and gives them a distended contour. This mass consists of blood cells, exfoliated mucosa, fecal matter, and extremely numerous coccidial forms of which the oücrsts or egg forms are especially apparent on microseopic examination. In older fowls, the ceca may be distended to a greater or less degree. In the majority of cases, the distention is marked and the tubes are firm to the touch over a considerable extent of their length. Hemorrhagic areas may show through the serous membrane, or the affected parts may appear pale and deadened. One cecum only may give evidence of morbid changes, but as a rule both are equally affected. On incision, the lumina are found packed with a solid necrotic mass of a grayish color and cheesy consistency. The mucous membrane is completely degenerated and forms a part of the caseated mass. On microseopic examination, the necrotic material appears as an amorphous mass in which mucus, cells in various stages of degeneration, food material and numerous coccidial eysts are incorporated. The mucous lining of the duodenum is invariably the seat of pathologic changes and is the portion of the intestine first affected. In many instances, this is the only part which shows lesions on post-mortem examination. The lining membrane is deeply congested, or hemorrhagic.

Diagnosis. Coceidiosis is readily diagnosed by a microscopic examination of the droppings of infected birds, or of the intestinal contents at autopsy. A small amount of the material is broken up in several drops of a 1 per cent potassium hydrate solution, normal salt solution, or water and about two drops of the mixture are placed on a glass slide and a cover glass applied. The coccidia usually are very numerous in advanced cases and are easily recognized in the oöcyst or resistant stage. They have an oval appearance with 
a central, rounded, darkened area surrounded by a wide, elear, transparent zone with double contoured border. Other stages of development may be observed, however, the oöcyst form is most easily recognized. Srmptomatically the disease may be confused with bacillary white diarrhea and aspergillosis in young chicks, and with tuberculosis, aspergillosis, and infections lenkemia in older fowls. It is differentiated from bacillary white diarrhea by the fact that deaths from the latter occur principally within the first three weeks of life, while coccidiosis usually appears at a later period. Aspergillosis eceurs more rarely and is generally restricted to a relatively small number of the flock. It is easily differentiated from coccidiosis on post-mortem examination by the localization of its lesions in the air passages, especially in the air sacs of the abdomen. Tuberculosis is readily distinguished by its characteristic nodular formations in the liver and intestines, or by microscopic examination. In infectious leukemia, the liver is enlarged and usually studded with small necrotic spots while the ceca are free of masses of caseated material.

Treatment. Attempts at treatment have not given very satisfactory results owing to the highly resistant powers of the organism during certain stages of its life cycle and to the position which it cecupies in the intestinal membranes. Crude catechu, recommended by Fantham, has, in our experience, proved more effective than other preparations which have been recommended. The most convenient method of administration is by means of the drinking water. The lumps should be broken up by grinding or pounding into a coarse powder and added to the water in the proportion of $1 / 3$ teaspconful to each gallon of water. This solution is to be kept constantly available to the flock while the disease is prevalent. Should signs of constipation develop, the entire flock may be given a dose of Epsom salts in the proportion of $1 / 3$ teaspoonful to each adult forwl. The salts can be dissolved in water and mixed in a mash. The purgative effect is greatest if the mash is fed in the morning when the crop contains a minimum amount of food. It is advisable when symptoms of eoceidiosis are first noticed to give the flock a dose of Epsom salts before the crude catechu treatment is started. Where catechu is not available, bichloride of mercury may be substituted. It is given in the drinking water in the strength of 1 to 6000 . Permanganate of potash 1 part in 500 parts of drinking water has also proved effective.

Prevention. Once this disease has been introduced, the most sat- 
isfactory method of combating it is through preventive measures against its spread. Birds showing symptoms should be isolated immediately, the houses thoroughly cleaned, and the floors, dropping boards and roosts soaked with carbolic acid in 5 per cent solution, or crude carbolic acid, or compound cresol, in 2 per cent solution. Other disinfectants, especially the coal tar products, may be substituted, provided sufficiently strong solutions are applied. The disinfectant may be spread by means of a spray pump or brush, but it is important that the places exposed to droppings should be covered by the fluid. During the course of an outbreak the droppings are to be removed frequently as it is through these that the infection is passed to healthy birds while feeding in contaminated pens. Runs to which diseased fowls have had access are highly dangerous to the non-infected and should be abandoned for a year at least if possible. These may be plowed and seeded, or if their use as runs is imperative the soil may be limed and plowed under. Since earth worms are credited with serving as hosts of the coccidium, stringent measures are necessary on badly infected premises, to accomplish complete eradication of the disease. Covering the ground with chloride of lime or unslacked lime at intervals may prove sufficient, especially where preventive measures have been adopted early and a minimum exposure to infested droppings is indicated. Fowls received from outside sourees, mnless known to be unexposed, should be quarantined for a period of three to four weeks before being placed in the flock.

\section{REFERENCES}

1. Fantham. Experimental studies in avian coccidiosis. Proc. Zool. Soc. London, Vol. 3, 1910, p. 708.

2. Fantham. Coccidiosis in British game birds and poultry. J. Econ. Biol., Vol. 6, 1911, p. 75.

3. Jowett. Coccidiosis of the fowl and calf. J. Comp. Path. and Therap., Vol. 24, 1911, p. 207.

4. Neyer and Crocker. Some experiments on medical treatment of coccidiosis in chickens. Am. Vet. Rev., Vol. 43, 1913, p. 497. 


\section{CHAPTER XIII}

\section{SPIROCHETOSIS AND RARE INFECTIOUS DISEASES}

\section{SPIROCHETOSIS}

Synonyms. Fowl fever, spirillosis of fowls, spirillose des poules, Hühnerspirillose.

Characterization. Spirochetosis is an acute, highly fatal, febrile, septicemic disease of birds caused by spirochetes and transmitted by fowl ticks.

Geographical distribution. The disease has been reported in North and South Africa, India, Australia, Russia, Hungary, Runmania, Bulgaria, Cyprus, South America and the West Indies.

The existence of the disease in the United States within the area of distribution of the fowl tick (See Fig. 53, p. 221) has been suspected by various writers, but so far as known its presence has not been definitely proven. Symptoms ascribed to excessive tick infestation, such as paralysis, drooping of wings, rufled feathers, loss of appetite and even death have suggested the idea that spirochetosis may be present.

Etiology. Spirochata (Treponema) gallinarum (S. marchouxi) causes the disease in fowls, while Spirochata anserinu has been identified as the cause of a similar infection in geese. Some consider $S$. anserina as identical with $S$. gallinarum.

The general shape of a fully developed spirochete is that of a narrow sinuous thread, in some cases reaching nearly 20 microns in length. The cells possess active motility. Spirochetes are generally regarded as protozoan organisms, although the belief that they are bacteria has many adherents. The organisms may be stained in blood films by fixing with osmic acid vapor, after which the films are hardened in absolute alcohol and stained with Giemsa or one of the modifications of that stain. Carbol fuchsin may be employed as a stain.

Noguchi has cultivated $S$. gallinarum in a special medium devised to provide suitable conditions. An infected bird is placed under ether anesthesia and blood is drawn aseptically from the heart. To prevent coagulation the blood is mixed with an equal amount of 
1.5 per cent solution of sodium citrate in a .9 per cent solution of sodium chlorid. A piece of kidney of a normal rabbit or a piece of pectoral muscle of a fowl is placed in a test tube. Ascitic fluid is added to make the column of fluid $10 \mathrm{~cm}$. high or about 10 to 15 c.c. To this is added a few drops of the infected blood. The fluid is covered with a layer of paraffin oil that has been autoclaved twice. Ascitic fluid is not always suitable and it may be necessary to try many lots.

The maximum growth is reached at about the fifth day, the cells being fully developed typical spirochetes. After the fifth day degeneration of the cells begins and proceeds slowly. The organism has been demonstrated to remain virulent for chickens through 13 generations of culture. However, under certain cultural conditions it may lose its virulence. The inoculation of birds with such avirulent strains induces a resistance to subsequent infection with a virulent strain.

Pathogenicity. Fowls, geese, ducks, guinea-fowls, turtle doves and sparrows have been reported as susceptible. Animals commonly employed in laboratory work are immune, but Levaditi reports that he produced a transitory spircchetosis in a rabbit lasting three or four days.

Poultrymen, when spirochetosis occurs, are apt to attribute losses to excessive tick infestation and to the resulting loss of blood without recognizing that an infectious disease is the cause of the losses.

The prevalence of the disease will coincide with the season that is most favorable to the multiplication of ticks, but deaths will occur at any time when susceptible stock is introduced, providing ticks are at all active.

Bevan notes that birds in infected flocks become immune. However, the birds previously immune will suceumb after the introduction of newly arrived susceptible birds starts the infection anew. It is possible that the passage through susceptible birds increases the virulence of the virus.

Upon introduction of infection into a flock hitherto uninfected, spirochetosis may be exceedingly fatal and exterminate a flock in a few days. Young birds are particularly susceptible.

The course of the experimentally produced disease varies according to the method of the introduction of the virus, the activity of the spirochetes and the receptivity of the bird. When the inoculation is made by means of the tick, after 6 to 8 days and sometimes more, the spirochetes are encountered in the circulation. If inocu- 
lation is made with virulent blood through the skin, the period of incubation is reduced to a period varying from 48 to 72 hours. The period of incubation is longer when the virus is placed on the skin superficially.

The disease is acquired easily through the mouth, especially if the virulence of the parasite has been raised by a number of passages.

Symptoms. V'ery commonly the disease occurs in such an acute form that birds apparently healthy at night are found dead under the roost in the morning. In the less rapidly fatal form there is dullness, ruttling of the feathers, somnolence and diarthea. The comb becomes pale as a result of anemia. The temperature reaches $110^{\circ}$ or $112^{\circ} \mathrm{F}$. but drops abruptly at the crisis, which coincides with the disappearance of the spirochetes from the circulating blood. During the course of the disease, there is a marked decrease in the number of red corpuseles and a leucocytosis characterized by increase in the number of polynuclear lencocytes. After the crisis, the polynuclear cells diminish and the mononuclear cells increase in number. The acute type of disease may be terminated by death in from four to five days. after the onset of symptoms. Death often occurs during convulsions.

The chronic type may follow the acute phase or appear independently. Death may not occur for fifteen days. Paralytic symptoms are noted. The wings may droop, or the head be twisted back, or the legs may be involved. Inability to use the claws is an early symptom of leg paralysis. There may be disturbances of gait, and the bird may appear knock kneed or bowlegged. Emaciation and anemia are also particularly characteristic of the chronic type.

Morbid anatomy. In birds dead of an acute attack, the spleen is found to be enlarged to several times normal. The liver also is greatly enlarged, shows fatty degeneration and sometimes focal necrosis. The other organs do not usually show marked lesions beyond the paleness of muscles, lungs and kidners resulting from the anemia. The intestines sometimes are congested, and show punctiform hemorrhages. In chronic cases on the other hand, the liver and spleen are smaller than normal.

The skin shows evidence of tick bites in the form of subentancons hemorrhagic areas.

Mieresenpic examination of the blood of a bird dead of the disease, or during life after the crisis, will not reveal spirochetes.

Life cycle of spirochetes. After the spirochete in the blood reaches a length of 16 to 19 microns it divides by a transwerse divi- 


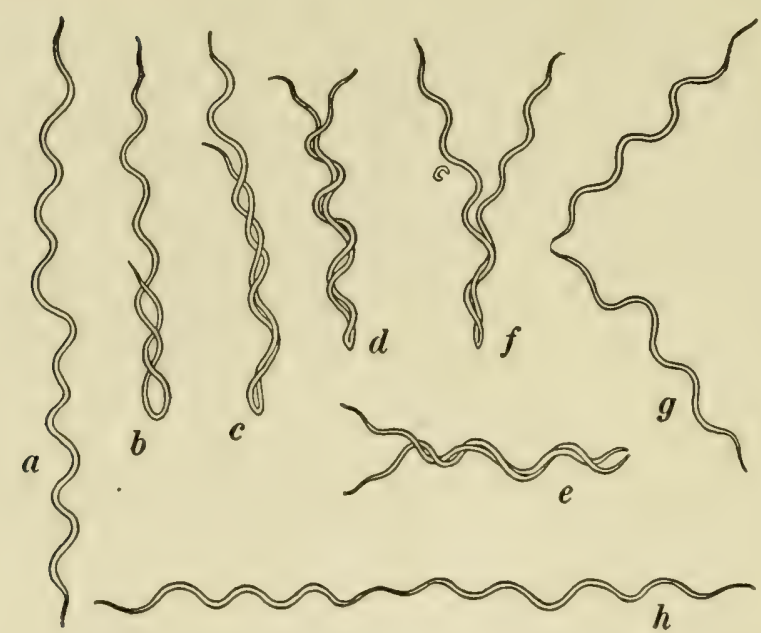

FIG. 14. Various stages in the flexions and transverse divisions of Treponema (Spirochota gallinarum). (Hindle)

sion. This is accomplished only after a series of movements consisting of doubling back on itself, coiling of the two portions, uncoiling and eventual separation. The process is illustrated in Fig. 14 , the successive stages of the process being indicated by the letters a to h. Sometimes the separation occurs as illustrated in e, but usually takes place after the position $h$ is reached. Whether or not transverse division takes place directly without previous flexion has not been definitely determined. The act of uncoiling and separation as illustrated in $d, f$, and $g$ of Fig. 14 very closely simulates a process of longitudinal division and has been erroneously so interpreted by some observers. The process of transverse division doubtless occurs repeatedly in the blood.

Penetration of red blood cells and invasion by spirochetes has been observed but seems to be uncommon.

At the time of the crisis of the disease, which is synchronous with the disappearance of spirchetes from the circulating blood, some spirochetes break up into a number of coccoid bodies in a manner analogous to the formation of spores within a bacterial cell. It is not certain that these coccoid bodies formed under the conditions in question redevelop into spirochetes within the blood of the fowl.

Some of the spircchetes taken into the tick with a feeding of fowl blood, penetrate the wall of the gut and gain access to the coelomic fluid which occupies the body cavity of ticks. After a short time. 


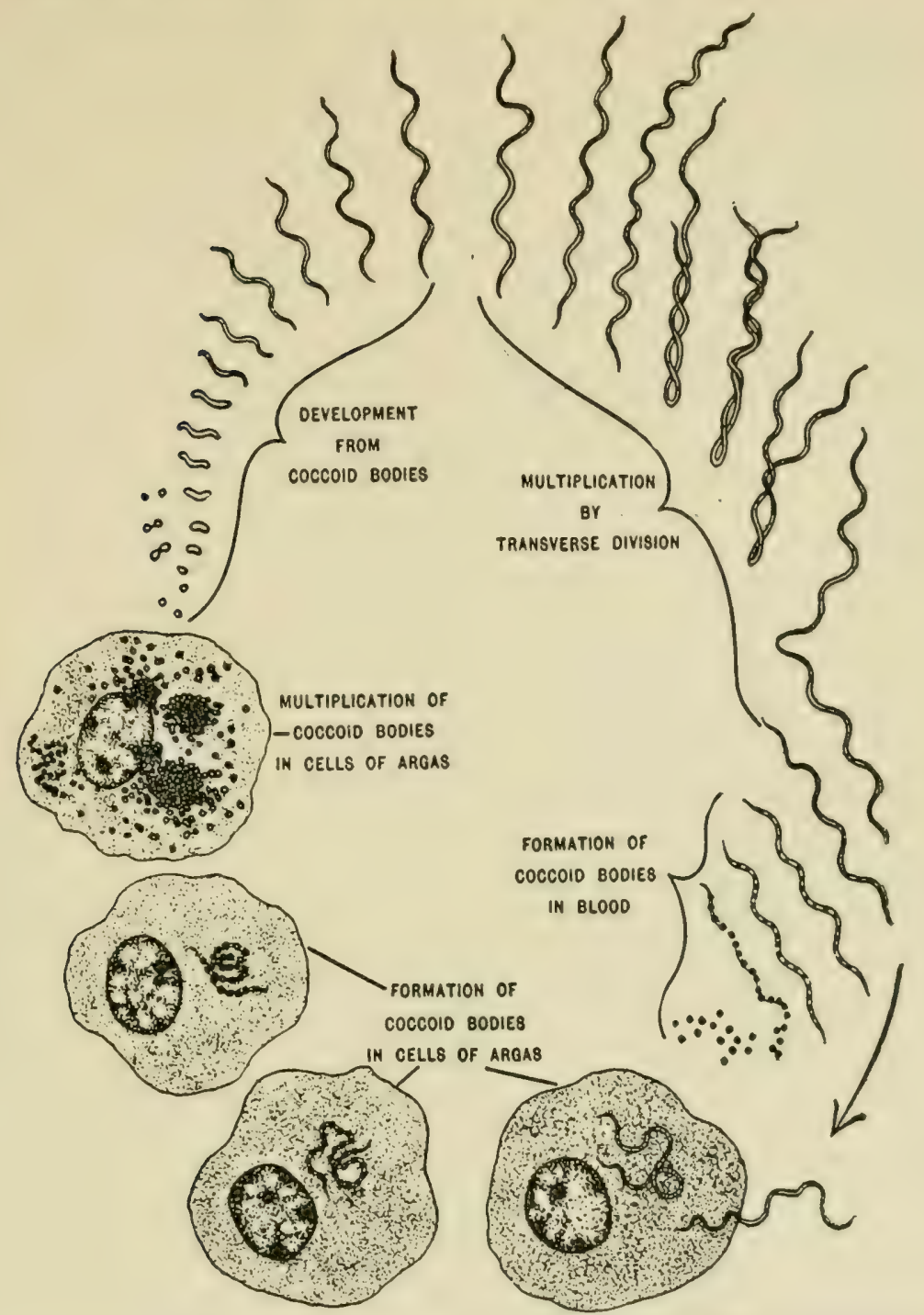

FiG. 15. The life-cycle of Treponema (Spirochata) gallinarum illustrated diagrammatically. (Hindle)

the spirochetes further penetrate into various organs of the tick, including the ovary and Malpighian tubules where they multiply by ordinary fission. In the cells of these organs the spirochetes break up into eccoid bodies. Other spirochetes remaining in the lumen of the gut also form coccoid bodies. These circumstanees re- 
sult in the infection of the Malpighian secretion and excrement with coccoid bodies.

While the salivary gland has been considered with reference to the part played by saliva in introducing infection into fowls, the observations tend to show that contamination of the tick-bite wound by coccoid bodies in excrement and coxal fluid, is responsible.

Coccoid bodies are capable of development into the typical spirochete by a process of gradual lengthening. This has been observed in the ticks but not in the blood of a fowl.

The egges of an infected tick eontain cocenid bodies and the progeny of such a tick is infective either by feeding on a fowl or when ingested by a fowl.

Ticks after feeding on infected blood are most liable to be infective when kept at a temperature of $30-35^{\circ} \mathrm{C}$. When ticks are kept at $15-18^{\circ} \mathrm{C}$, the spirochetes disappear from the alimentary tract, and such ticks do not transmit the infection.

Differential diagnosis. The various septicemias of fowls, more particularly fowl cholera, might realily cause symptoms and lesions simulating spirochetosis. Microscopic examination of stained blood smear's should furnish conclusive evidence by showing whether the septicemia is eaused by spirochetes or by bacteria. The limited area of the United States in which ticks ocenr, see p. 221 , should be borne in mind.

Treatment. Dodd reports that $1 / 5$ grain of soamin dissolved in one c.c. of sterile water and injected intramuscularly, modified and shortened the attacks with rapid and complete recovery. Uhlenhuth gave atoxyl in an average dose of 5 centigrams at the time of infection or two days later. It prevented infection or cured but the blood remained infective.

Haner conchudes that salvarsan is capable of destroying spirochetes in the body. The curative action of this substance is established in all cases on the day of treatment and acts in a remarkable mamner even after the use of a limited amount of salvarsan. Even in cases where the treatment was first given on the fourth day after infection, when the bird was somnolent and when the blood was swarming with spircchetes, one injection of the agent in not too small a dose led to striking improvement and recovery. The immunity which salvarsan confers upon birds protected with it, is of high degree and of long duration.

Haner experimented on fowls with a wide variety of doses of the drug. The lowest lethal dose was found to be $.3 \mathrm{gram}$ and .15 
gram was the maximum dose tolerated. The curative dose on the second day of infection when spirochetes were observed in the blond, varied from .02 to .003 gram per kilo of body weight. Even the lowest dose caused the disappearance of spirochetes from the circulation and the birds recovered. Birds treated on the third day of infection received doses varying from .03 gram to .0025 gram per kilo of body weight. The lowest dose that miformly caused the disappearance of the spirochetes was .005 gram per kilo. A dose of .05 gram per kilo was effective when administered to birds on the fifth day of infection. Aragào recommends atoxyl in a dose of .03 gram, salvarsan .0035 gram and neosalvarsan in .0015 gram per kilo of body weight.

Immunization. An attack of spirochetosis confers a certain amount of immunity. The blood serum of such a bird possesses strong immunizing properties and shows marked agglutinative action in vitro.

Aragao induces immunity by injecting a vaccine prepared from the blood of infected fowls. On the fifth day of the infection the blood is drawn aseptically into flasks holding 300 c.c. each and defibrinated by shaking with shot. Blood drawings from the various fowls are mixed to secure a uniform distribution. The lefibrinated blood is distributed in quantities of 50 c.c. each in flasks of a capacity of 125 c.c. The flasks are plugged with cotton which has been dipped in formalin. The fluid is left thus exposed to the vapor of formalin for eight days and is shaken occasionally during that period. After carrying out suitable tests for sterility, it is injected subcutaneously in one c.c. doses for immunizing birds before introduction into infected flocks. A similar product is prepared by Aragão by mixing defibrinated blood and glycerine in equal parts, omitting the formalin vapor treatment. It is used in two c.c. doses and is preferred to the one prepared by using formalin. A single injection is employed, for subsequent attacks by ticks in an infected flock are relied upon to strengthen the immunity. In uninfected flocks where the vaccination is employed as a protection against imported infection only, revaccination is recommended once a year.

Marchoux and Salimbini have observed that the virulence of the spirillum in blood is greatly diminished or lost after a period of about 48 hours. They produce successful vaccination by using virulent blood held for two to four days, or after heating at $55^{\circ} \mathrm{C}$. for five to ten minutes. 
Control of spirochetosis. The relation of fowl ticks to the spread of the disease is so direct, that the destruction of ticks is of necessity the first measure to be employed in combating the disease. See p. 223. Fowls not infested with ticks, but liable to become so, might be immunized with Aragào's vaccine. Susceptible birds before introduction into tick infected localities might be so immunized.

\section{RARE INFECTIOUS DISEASES}

RABIES

Several writers report the occurrence of rabies among fowls. Affected birds show restlessness and great fright. The feathers are ruffled and the bird attacks its fellows, other domestic animals and even man.

Autopsy findings reveal no marked lesions. Injuries of the skin, foreign bodies in the gizzard together with congestion of the kidneys and intestines are conditions most frequently found.

The prophylactic measures indicated are isolation of birds during outbreaks of rabies with slaughter of infected birds.

Intracranial inoculation of fowls with rabies virus causes paralysis of the feet and neck, associated with uncertain gait.

\section{FOOT AND MOUTII DISEASE}

The occurrence of foot and mouth disease has been observed in fowls, waterfowl and pigeons but a few times. The lesions have been reported as occurring on the skin of the head, on the buccal mucous membrane and about the feet.

Ehrhardt states that this disease is very rarely transmitted to fowls from cloven hoofed animals, for birds show a very high resistance to both natural and artificial infection. The disease is manifested by the occurrence of vesicles on the appendages of the head, on the mucous membrane of the mouth and throat as well as on the feet. In waterfowl the lesions appear mostly on the webs of the toes, and on the mucosa of the mouth and throat. In most cases the course of the disease is favorable and the lesions heal spontaneously in from eight to fourteen days without treatment. As a result of the cessation of eating, the affected birds appear weak and cease laying. In severe eases lameness and fever are observed followed by decline and death. 


\section{MALTA FEVEIR IN FOWLS}

Dubois reports the occurrence of this disease in fowls. The outbreak lasted three months, with a mortality of 70 per cent.

Symptoms. Birds of all ages are affected. The disease presents two clinical forms: an acute or subacute form in which the duration is 8 to 10 days at the most, and a fulminating form lasting only a few hours.

In the acute form the affected birds appear feeble, walk with difficulty and display inappetence. After three or four hours the birds stop moving and appear depressed. The wings drop, the birds allow themselves to be eaught easily, and sometimes show diarrhea with green discharges. There is extreme emaciation at the last.

In the fulminating type few characteristic signs are observed. There is only weakness and depression. Some birds die suddenly without having shown symptoms.

Morbid anatomy. In the fulminating type the lesions consist of ecchymoses on the lungs, a marked hypertrophy of the spleen and a certain degree of congestion of the liver.

Incculations made with the liver, the spleen, and heart blood of sick birds have given negative results. Likewise, inoculation of rabbit, guinea pig and pigeon with the pulp of the spleen and heart blood of sick birds has always failed to affect these animals.

The writer determined the agglutinative properties against $M$. melitensis possessed by the blood of the affected birds. Of 8 fowls $2-5$ years old, 3 presented a positive reaction; of 9 birds $2-4$ months old, 5 gave positive reaction.

\section{ANTHRAX}

Dawson points out that chickens may contract anthrax by eating the carcass of an animal dead of that disease. The disease runs a rapid and fatal course within 24 hours. The affected bird shows fever, high temperature, weakness, tremors and convulsions, together with bloody fecal discharges. Swellings may oceur on the coml, wattles, sides of the head, in the mouth or on the feet.

Möllhoff concludes that birds are more or less susceptible to inoculation with anthrax. Exception is made in the case of hens, which in his experiments showed high resistance. Out of sixteen hens exposed by subcutaneous, intramuscular and intraocular injection, with doses as high as 2 c.c. or by feeding, only one suc- 
cumbed. This bird before inoculation was observed to be highly emaciated and anemic. Two geese receiving subcutaneous injections of 4 c.c. resisted infection. Of 16 pigeons inoculated, 7 contracted anthrax. Other birds such as ducks, sparrows, canaries, jays, hawks and crows, were very susceptible to inoculation. That writer makes a distinction between susceptibility to anthrax by inoculation, and susceptibility to the natural spontaneous infection. He knows of no instance of the latter.

Möllhoff concludes that the resistance of the hen to anthrax consists of action of the body fluids or lymph by virtue of strongly bactericidal materials contained in them. The anthrax bacilli in the subcutaneous tissues are killed in a short time by the bactericidal action of the lymph, so that no local development nor general infection can occur.

The origin of this bactericidal material of the lymph, especially with reference to whether or not it originated in the leucocytes, conld not be determined. Phagocytosis is not of decisive importance in connection with the destruction of anthrax infection in the hen. The resistance of hens against anthrax does not depend upon their high body temperature.

Anthrax occurs in the ostrich as a common natural infection and is discussed in Chapter XVIII.

\section{REFERENCES}

1. Aragào. Espirochetose (treponemose) das Gallinhas. Rev. de Vet. e Zootech. (Rio de Janeiro), Vol. 7, 1917, p. 3.

2. Bevan. Spirochetosis of fowls in Southern Rhodesia. J. Comp. Path. and Ther., Vol. 21, 1908, p. 43.

3. Dawson. Anthrax with special reference to the production of immunity. U. S. Dep. Agr. Bureau Animal Indust. Bull. 13.

4. Dubois. La fièvre de Malto chez les poules. Rev. Vet., Vol. 67, 1910 , p. 490 .

5. Ehrhardt. Die Krankheiten des hausgeflügels. 3. aufl. Aarau: E. Wirz. 1914.

6. Hauer. Untersuchungen über die Wirkung des Mittels 606 auf die Hühnerspirillose. Centralbl. f. Bakteriol. (Etc.), 1 Abt. Orig., Bd. 62, 1912 , p. 477.

7. Hindle. On the life-cycle of spirochæta gallinarum. Parasitology, Vol. 4, 1911, p. 463.

8. Jowett. Fowl spirochetosis at Cape Town. Vet. Jour., Vol. 18, 1911, p. 240.

9. Mrarchoux et Salimbini. La spirillose des poules. Ann. de l'Inst. Pasteur, T. 17, 1903, p. 570.

10. Möllhoff. Untersuchungen über die Empfanglichkeit des Geflügels 


\section{SPIROCHETOSIS AND RARE INFECTIOUS DISEASES}

für Milzbrand und über die Gründe der Resistenz des Huhner gegen diese Krankheit. Inaug. Diss. Bern.

11. Noguchi. Cultivation of spirochæta gallinarum. J. Exper. MY, Vol. 16, 1912, p. 620.

12. Pereira. A Espirochetose das Gallinhas. Rev. de Vet. e Zootech., Vol. VI, 1916, p. 327. 


\section{CHAPTER XIV}

\section{LEUKEMIA AND PSEUDO LEUKEMIA}

Characterization. Leukemia in fowls is a disease of the blood, and blood forming organs. It is marked by changes in the composition of the blood manifested principally by an enormous increase in the number of lencocytes and an accompanying decrease in the number of erythrocytes. Besides these changes, lymphoid enlargement of the liver, spleen and kidneys is usually to be observed. Pseudo leukemia is a term designating a condition in which lymphoid tissue is present in the various organs, without changes in the circulating blood. The two conditions are very closely related, if indeed they are not different stages of the same process.

History. The disease in fowls was first recognized by Warthin in 1907 and soon after by Ellerman and Bang. Butterfield in 1905 and Yutaka Kon in 1907 very likely observed cases of leukemia, but did not examine the blood. Pickens presents a thorough survey of the literature of the disease accompanied by original observations on a series of cases. The disease has been reported in Denmark, Germany and the United States.

Etiology. Ellerman and Bang and also Yutaka Kon found protozoa-like bodies in the organs and bone marrow of affected fowls. The significance of these bodies has not been definitely determined. In typical cases of leukemia encountered by the present writers, attempts to isolate an organism from the blood and parenchymatous organs have given negative results. The fact that Ellerman and Bang succeeded in transmitting leukemia to fowls by intravenous and intraperitoneal injection of a cell free filtrate of infected exudates, points to an ultra-microseopic virus as the causative agent.

The disease is readily transmitted by non-filtered organ suspension. The virus is present in all affected organs, but the infective properties of these organs are lost in a few days after death.

There are three principal theories as to the etiology of the disease in man which are reflected by various writers in the interpretation of lesions in the fowl: 1. The lesions of leukemia are a simple hyperplasia. 2. Leukemia is a neoplasm. (See lymphoma). 3. Lenkemia is a result of the multiplication of an infective agent. 
Pathogenicity. Leukemia has not been reported as oceurring in pigeons, turkeys or guinea-fowls and experimental efforts have failed to produce the disease in these birds. Fowls appear insusceptible to subeutaneous inoculation but are infected in about 50 per cent of cases through intravenous or intraperitoneal injection of a suspension of the organs or of the blood of affected fowls. Ellerman and Bang observed that inoculation may produce either leukemia or pseudo leukemia.

Course. The duration of the disease is variable. The period of ineubation ranges from about 2 to 8 weeks. Death may result within several days after the appearanee of symptoms or may be delayed for one to three months. Recovery is only rarely observed.

Symptoms. While birds may die suddenly from an acute attack, the manifestations of leukemia are usually of a chronic character. There is observed a progressive emaciation with dull, depressed appearance and noticeable weakness. In some cases the abdomen droops. The appetite generally remains good in the chronic cases but may fail in acute cases. The comb, wattles and skin are pale as a result of the diminution of the red blood cells and increase of the white cells. Blood when drawn presents a pale red color and fails to clot readily, while normal fowl blood clots very rapidly.

Morbid anatomy. The carcass has an anemic appearance. U'sually the liver and spleen show the most marked changes. The kidneys are at times also severely involved. The liver may be slightly enlarged, congested and sprinkled with minute whitish points, or it may be markedly enlarged and congested. Not infrequently rupture of the liver resulting in internal hemorrhage is observed. The enlarged liver may appear much darkened, soft and pulpy in more acute cases. In cases of longer duration, this organ is greatly enlarged and pale in appearance or else mottled and sprinkled with grayish white spots. It has a firm consistency and rigid contour. On cut section the tissue appears bloodless. The spleen generally shows changes in conformity with those of the liver. It may be only slightly congested or may be greatly enlarged and congested. As in the case of the liver, the consistency varies from soft and pulpy to a firm, compact mass. The color is bright mahogany in the case of severe congestion and sometimes grayish and hrownish in spots. The kidneys may be several times the normal size, congested in the early stage or firm and pale at a later period. The intestine appears pale on the serous surface but areas if engestion 
may be present on the mucous membrane, especially in the duodenum.

Microscopically, the blood is found to contain a greatly increased proportion of leucocytes to red blood cells. The normal number of leucocytes per cubic millimeter is approximately 30,000 . This is increased in leukemia to from 100,000 to 500,000 per cubic millimeter, while the normal number of red blood corpuscles, approximately $3,000,000$ per cubic millimeter is reduced to nearly $1,000,000$ per cubic millimeter. The normal proportion of about 1 white cell to 100 red cells is thus changed to 1 to 2 or 1 to 3 . The mononuclear leucocytes increase more rapidly than the polynuclear leucocytes, or the smaller lymphocytes. Round nucleated red blood cells representing normoblasts and megaloblasts are to be seen in stained preparations. The hemoglobin content decreases from a normal of $50-65^{\circ}$ to $15-20^{\circ}$. The blood is pale red in color and clots with difficulty. The liver, spleen and kidneys are found engorged with lencocytes. These fill the smaller capillaries and occupy a perivascular position in the parenchyma. There is a hyperplasia of the cells of the bone marrow and spleen, the latter being an important source of the lencocytes which infiltrate the liver and kidneys. Degenerative changes are observed in the affected organs.

Diagnosis. The presence of the disease is indicated by the anemic condition of the fowl, the enlarged liver and spleen, and the absence of a demonstrable microorganism in these tissues. This latter feature distinguishes it from bacterial septicemias in which the liver and spleen may be swollen and congested. Microscopical examination of the blood is of the greatest value in differentiating from other affections. In simple leucocytosis accompanying infectious diseases, the preportion of white to red blood cells while at times approaching 1-25 is not as striking as in infectious leukemia. Also the absence of myelocytes is noted in the former disease. In leukemia the white cells in the blood have approximately the following percentage relation: Mononuclear 70 per cent, small lymphocytes 20 per cent and eosinophiles 6 per cent. The diagnosis of pseudo leukemia during life presents great difficulties.

Treatment. As in the case of other deep seated diseases of a malignant trpe, treatment of individuals is unfortunately of questionable value. There is no definite method of treatment known which alters the course of the disease so as to bring about recovery. Potassium icdide in doses of 3 to 7 grams daily, and careful nursing together with the administration of tonies, such as gentian, nux vomica 
and iron, may be of benefit in mild forms of the disease. Arsenic might also prove beneficial.

Prevention. In sporadic cases of leukemia, no special precautions against the spread of the disease can be recommended other than the usual precaution of isolating or destroying the sick bird. Where several cases develop in the course of a few days, all birds showing the slightest abnormal appearance should be immediately separated from the flock. The droppings, litter, etc., should be removed and the quarters thoroughly disinfected. The drinking water may be medicated with permanganate of potash up to a 1-1000 solution.

Pseudo leukemia. Ellerman and Bang regard this affection as being indicated by the same lesions as the true leukemia. The enlargement of the spleen and of the liver is often very noticeable. The blood is not leukemic. They consider it very probable that this disease has the same cause as true leukemia for cases of psendo leukemia are encountered in outbreaks of the true disease. Inoculation of a hen with organs from a case of pseudo leukemia caused alteration of organs, typical of lenkemia, with a blood picture suggesting the beginning stage of leukemia.

\section{REFERENCES}

1. Pickens. Leukemia and pseudo leukemia. Rept. N. Y. State Vet. Col. '1915-1916, p. 226.

2. Ellerman u Bang. Experimentelle leukämie bei Hühnern. Cen. tralbl. f. Balkteriol. (Etc.), 1 Abt. Orig., Bd. 46, 1908, S. 595.

3. Warthin. Leukemia of the common fowl. J. Inf. Dis., Vol. 4, 1907, p. 309 . 


\title{
CHAPTER XV
}

\author{
GENERAL DISEASES
}

GOUT

Characterization. Gout is a disease characterized by the increase of uric acid content of the blood and the deposition of uric acid salts in the joints as well as in various organs, especially on the serous membranes. The disease apparently does not oceur in birds living free but is frequent in cage birds of zoological gardens and in all sorts of domestic birds. Among these, hens are affected most frequently, waterfowl less frequently, and pigeons least.

Etiology. Gout appears to depend for its causation, upon the use of feeds rich in protein in the absence of which many breeders believe that it will not occur. Furthermore, a one sided diet, close confinement, lack of exercise and heredity appear to be predisposing factors.

Two types of gout are recognized, a rare gouty arthritis and a more common visceral gout.

Visceral gout. In visceral gout the serous membranes of the chest and abdominal cavities are covered with a layer of uric acid crystals of greater or less thickness. In the pericardium which is affected with gout with greatest frequency, there is often a deposition of a layer of uric acid salts 1 to $2 \mathrm{~mm}$. in thickness.

Articular gout. This type preponderates in the joints of the feet as well as in those of the wings. The joints are thickened and inflamed, while the joint capsule shows a doughy swelling. Knotty swellings varying in size up to that of a cherry, occur in the vicinity and break outwards, discharging white or grayish yellow masses which consist of uric acid salts.

Hebrant and Antoine describe a case showing unusually marked articular lesions. The subject from which the legs were taken was a cock two years old. The bird otherwise appeared in good health. The feed had been corn and wheat. The subject could not walk for it was unable to stand upon the feet.

The lesions consist of a number of tumors, some of which are as large as a pea. They are particularly numerous on the inferior 


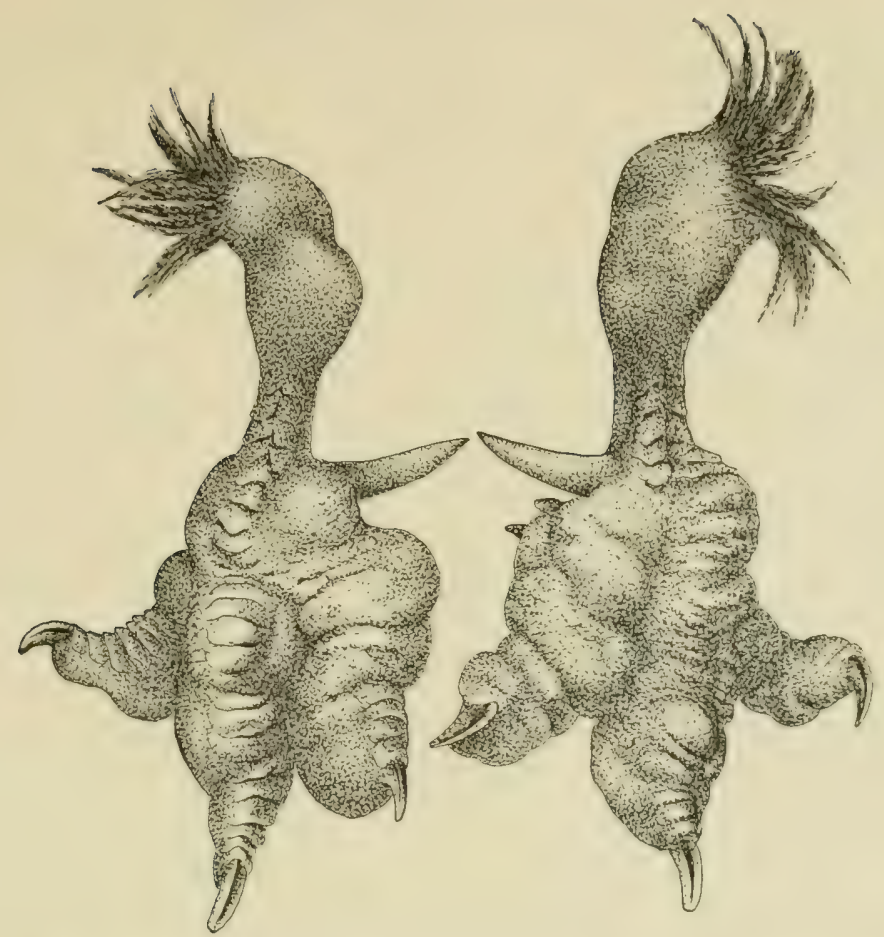

FIG. 16. Legs of cock showing lesions of articular gout. (Drawn from plıotograph by Hebrant and Antoine)

surface of the digital region in the vicinity of the articulations. Because of interference with circulation the tumor's have a gravish yellow color. Some of the tumors fluctuate and their appearance is suggestive of peri-articular abscesses. On incision the tumor's are found to contain a whitish, creamy, pasty mass consisting of crystals of uric acid. The majority of the tumors are located in the periarticular connective tissue and a small number communicate with the articulation.

Gout produced experimentally. In order to determine what influence is exerted by protein on nutrition and the occurrence of gout, Kionka fed hens exclusively on horse meat that had been minced, and freed completely from fat. They were allowed to drink water as desired and soon became accustomed to the compulsory diet. Within a period varying from three to fifteen months they all became affected with gout appearing in three forms.

In every case at first the gait became uncertain and diffienlt and the affected birds fell often while walking. In the first trpe of 
the disease the pain seemed to appear suddenly and on account of it the bird crouched and stopped eating. This coincided with the appearance of isolated gouty nodules. Gradually the periods of pain became more frequent, the appetite was entirely lost and death followed. The deposits of uric acid crystals in the joints were not especially marked. In the second form the attacks of pain did not appear in so typical a manner, but the nodules were larger and occurred principally on the joint capsules and tendon sheaths of the wings. The third type was visceral and the deposits were limited principally to the serous membranes and the kidneys. The experiment proves the dietetic origin of gout.

Diagnosis. The symptoms of the joint inflammation are distinct: occasional loss of appetite, swelling of the joints, disinclination to move. At other intervals, the appetite is good and the swellings decrease. At last there is no eating, inability to move, emaciation, rapid loss of strength and death.

Visceral gout cannot be recognized in the living bird.

Treatment. Operative treatment is of little use but may be resorted to in valuable birds. The joint tubercles should be opened to evacuate the contents and an antiseptic dressing applied.

As soon as the cause of death is revealed by autopsy an appropriate diet should be provided for the other birds kept under similar conditions. The feeding of a rich proteid diet should be restricted, with a fast of one day a week. Grain feed, abundant green feed or roots should be provided.

\section{RACHITIS}

Characterization. The disease consists of disturbance of normal bone formation of which the most prominent evidence is the deficient deposition of calcium salts. While the course of the disease is not well understood, it seems clear that it is caused by a general interference with nutrition. Deficiency of intake of lime is concerned. Rachitis is a disease of young birds and seldom oceurs in birds over half a year old. It is limited almost exclusively to fowls and oceurs seldom in the turkey, waterfowl and pigeons.

Symptoms. The birds show exhaustion, difficulty in locomotion, inappetence, diarrhea, paleness of the mucose and emaciation.

Morbid anatomy. Autopsy reveals intestinal catarrh and lesions of the bones. The large bones of the extremities are bent as 
is also the breast bone which becomes $\mathrm{S}$ shaped. The present writers conducted an autopsy on a young turkey in which the skcleton was apparently wholly lacking in mineral constituents. The larger bones could be bent with the fingers and sliced with a knife. The bird was reported as being one of a number affected in a similar manner.

Treatment. Normal feeding should be the first matter considered. Birds should be supplied with dry, warm quarters, with access to grass if possible. Feed coarsely cracked grain, cracklings, or meat meal and burned oyster shells. Calcium phosphate may be administered in doses of .5 to 2.0 grams per bird, according to size. Small amounts of sulphate of iron may be given by putting this material in the drinking water in the proportion of one or two parts per 1000 parts of water. No treatment should be attempted on those birds liable to become cripples. Such should be killed.

\section{LEG WEAKNESS}

Leg weakness is a term designating unsteadiness of gait, which may be followed by total inability to stand on the legs. At first the bird otherwise appears healthy, but soon shows the effect of inability to compete with its fellows for food. If the condition constitutes paralysis, "limberneck" may also be observed. Leg weakness also is observed in rachitis. It occurs in well fed young growing birds under conditions not well understood. Tnder such circumstances the ration should be reduced and green feed should be supplied. At other times it occurs in closely confined birds kept on a monotonous diet. In such cases trouble disappears when birds have access to the soil with consequent variety of diet.

Polvneuritis of fowls, a condition induced by feeding polished rice or similar products, is the one form of leg weakness, the etiology of which is understood. The affection may be produced experimentally in from fifteen to twenty-five days by feeding a diet limited exclusively to polished rice, while a diet of natural unpolished rice dnes not produce this result. The condition of the fowl designated polyneuritis is generally regarded as identical with the disease of man called beri beri, which latter is common among people living almost exclusively on polished rice. It is evident that the rice hull contains sulbstances, the absence of which induces neuritis. These have been designated vitamines.

Vitamines of this character are not restricted to rice. It has 
been demonstrated that they are found in a large number of natural foodstuffs. They are very common in the seeds of plants such as cereals. In these, the vitamines are mainly deposited in the germ or embryo and to a less extent in the bran. White wheat flour is deficient in vitamines and is capable of producing polyneuritis.

The facts observed during experimentation on fowls in connection with the study of the etiology of beri beri of man, suggest the possibility that in isolated instances, leg weakness of chickens may be induced by a similar cause.

\section{LIMBERNECK}

Limberneck is a symptom resulting from partial or complete loss of control of the muscles of the neck. Probably various causes such as digestive disturbances, intestinal parasites and the eating of spoiled meat may cause this symptom. Feeding on maggots from decaying meat will cause limberneck.

According to Dickson, chickens when fed botulinus toxin, beeme dull and inactive, refuse to eat, remain quiet in one place with the feathers ruffled. They gradually develop weakness of the leg's, wings and neck so that they are unable to stand. The wings droop and the beak or the side of the head rests upon the floor of the cage. Death occurs within 24 hours after feeding.

Treatment consists of administering castor oil. Preventive measures should include precautions against allowing birds access to decaying meat.

\section{DISEASES OF THE ALIMENTARY TRACT}

PIP

The condition commonly termed "pip" does not represent a specific disease, but is usually found associated with avian diphtheria. Then the nasal passages become closed by the presence of mucous secretion or exudate, the fowl is compelled to breathe through the mouth. The constant passage of air over the tongue tends to dry and harden it at the tip. The dry, horny covering contracts and presses upon the soft tissues beneath, causing the fowl much annoyance. The hardened layer may partially separate from the soft tissues and expose a raw, inflamed surface. The practice among some poultrymen of removing the horny tip should be discouraged since it only increases the discomfort of the fowl by leaving a raw, sensitive surface exposed to foreign irritants and microorganisms. 
To alleviate this condition the primary cause should be remored. An effort should be made to keep the nostrils open by removing exudates and syringing the parts with an antiseptic solution such as 3 per eent boracic acid or 2 per cent permanganate of potash. If the disease which is responsible for "pip" is successfully treated the condition will disappear.

When the symptom is noticed it is advisable to rub the tongue with some substance such as vaseline, cottonseed oil or glycerine.

\section{THRUSH}

Thrush or soor has been observed to affect the month and crop of fowls, pigeons and turkeys. The affection is characterized by the formation of grayish white or yellowish colored patches adhering to the mucous membrane without inflammatory changes in the latter. Severe involvement causes death. The organism causing the infection is designated Oidium (Saccharomyces) albicans. Treatment of oral lesions may consist of the application of bichloride of mercury in a solution of $1: 1000$. The crop may be irrigated with boric acid solution as in catarrh of the crop. Authoritative

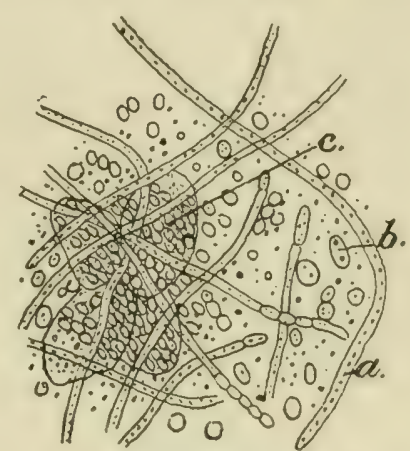

FIG. 17. Oidium albicans, a, cylindrical mycelium; b, spore; c, epithelial cells reports of the occurrence of the disease in fowls are rare.

\section{CATARRH OF THE CROP}

This is a mild inflammation of the lining membrane of the crop due to excessive irritation by ingestion of material of an irritating nature, or by the products of fermentation when food for one reason or another, stays in the crop for an unusual length of time. It occurs in cases of impaction and may also be associated with certain infectious diseases. Parasites at times may cause the condition.

Symptoms. The crop is usually distended with food or gas. The fowl appears sluggish and the appetite is diminished or lost. Occasionally the beak is opened wide as if to eatch the breath or as if attempting to vomit. The head and neck also describe tossing movements. Later a gray, sour, ill-smelling fluid is discharged from 
the beak or from the nostrils. Without treatment the strength of the subject fails rapidly.

When the disease has existed for some time in a chronic mild form it may assume the condition designated hanging crop. In such cases the musculature of the crop has been overstretched, so that after recovery from the catarrh, the organ does not return to its normal position.

Treatment. This consists of the removal of the contents of the crop as soon as symptoms are noticed. If the contents are soft the fowl may be held head downward and the crop manipulated in such a manner that the material will be passed back through the esophagus to the mouth. This is relatively easy owing to the large diameter of the esophagus of fowls. Should the crop contents be hard or dry they may be softened by giving the bird several teaspoonfuls of water before attempting to remove the material. When the crop is empty, administer 1 teaspoonful of castor oil. Feed should be withheld for about 24 hours, but buttermilk, or sour milk may be freely given together with water. A small amount of bicarbonate of soda may be administered and the bird should have access to charcoal in case fermentation is noticeable. Fowls showing frequent attacks or chronic eatarrh of the crop should not be retained.

\section{IMPACTION OF THE CROP}

Feeding with dry grains such as corn, peas, oats, or with bran may cause a distention of the crop. Various foreign bodies may cause the same condition. The enlargement of the organ will first attract attention. If not remedied, the condition will cause death of fowls in a few days, and in waterfowl in a few hours, because of pressure on the trachea. Massage may permit the removal of material through the beak, but usually incision of the crop is indicated.

\section{INFLAMIMATION OF THE CROP IN PIGEONS}

The crop of the pigeon secretes a milk-like fluid for the nourishment of the young. If the parent birds lose their young while the crop is secreting this fluid, an inflammation of the crop glands frequently results and the organ feels hard and solid. The disease may be quickly remedied by substituting another squab. Only one should be sulstituted for often pigeons will not adopt two young ones, but kill them and throw them out of the nest. Besides, if two 
were taken, the parents thus robbed of their young, would develop the same trouble. If such a procedure is not possible, the sick ones should be isolated without food. They may be allowed water slightly acidulated with vinegar. If abscess-like lesions oceur on the breast they should be opened.

\section{GASTRITIS}

Gastritis is an inflammation of the mucous membrane of the proventriculus or first stomach. It may result from digestive disturbances but is generally associated with the presence of strong irritants such as certain mineral poisons, or with infectious diseases of a septicemic type. Inflammation of the crop may extend to the proventriculus. In itself, it is not of great importance.

Symptoms. The diagnosis of gastritis is diffieult. If no symptoms of disease are noticed other than a loss, of appetite and a sluggish appearance, simple gastritis may be suspected. When associated with other diseases the symptoms which accompany these will be observed.

Treatment. The affected fowl should be given 2 teaspoonfuls of eastor nil to which 10 drops of oil of turpentine have been added. Feed lightly for a time. Buttermilk or sour milk may be freely given. Give 30 drops of tincture of gentian twice daily.

\section{INDIGESTION}

Mrégnin distinguishes four sorts of indigestion in birds. It may result from lack of quality or appropriate volume of food or by ingestion of foreign bodies. Thus pigeons which naturally are granivorous will suffer if circumstances necessitate their consuming herbage. Birds in zoological gardens are liable to suffer from lack of appropriate food. Storks, herons, swans, geese and ducks in the winter and spring failing to obtain their natural food such as molluses, slugs, etc., attempt to cheat the appetite with plants or aquatic mosses. These materials are to them true foreign bodies which cause fatal indigestion. It is the same in the case of the small insectivorous or semi-insectivorous birds. When it is attempted to feed them exclusively on grains or vegetable mashes they succumb to the diet.

Grains too large for the digestive organs of a bird act like a foreign body and cause fatal indigestion. Parroquets and young pheasants may be killed in this way by grains of maize. 
Overloading the digestive organs is a second general cause of indigestion. Dry grain which is inviting to certain birds like the gallinacer is less suitable to others such as palmipeds which are accustomed to soaking their food in water. Thus such birds may readily orerload their stomachs with dry grain which may swell and cause death.

Indigestion may be caused by lack of silicious gravel in the gizzard. This material is indispensable to granivorous birds to triturate the grain in that organ. Omnivorous birds likewise need grit. In the absence of such material, trituration is not accomplished and grain accumulates in the gizzard. Thus death may be caused by indigestion occasioned by repletion, or whole grains may pass into the intestine and cause a fatal inflammation. This may occur among a large number of birds. The present writers have observed severe enteritis in a wild goose occasioned by heavy snowfall and prolonged freezing of a pond, which circumstances prevented access to grit. In the absence of grit of suitable size, birds will attempt to swallow articles entirely too large, with fatal results.

There is in the opinion of Mégnin, an indigestion resulting from lack of food, or disease of hunger. Domesticated birds may accidentally be deprived of food or may be prevented from eating by stronger companions.

Indigestion in birds is rarely recognized during life and is only encountered at autopsy. That caused by overloading may be recognized and treated with a little olive oil. Prevention along the line of providing suitable food and grit must be relied upon chiefly.

\section{INDIGESTION IN PARROTS}

The affection may result from a variety of causes such as improper feeding and failure to provide grit which impairs the natural function of the gizzard. The affected bird yawns, displays loss of appetite and occasionally manifests nervous svmptoms. Correction of feeding is of first importance. Castor oil in a dose of from 5 to 10 minims may be administered. Bicarbonate of soda in 5 grain doses may be given in the drinking water for several days. Nux vomica is indicated.

\section{ENTERITIS}

Enteritis is an inflammation of the mucous membrane of the intestine. It is perhaps the most common symptom observed in connection with poultry diseases. 
Etiology. Enteritis is associated with a large number of important diseases. It varies in the intensity of the inflammation with different affections. In fowl cholera, fowl plague and several other septicemias the inflammation is very severe, while in fowl typhoid and infectious leukemia the mucous membrane may show little or no congestion. Enteritis is associated with such protozoal diseases as coccidiosis and infectious entero-hepatitis, and with intestinal worm infestation.

Mineral poisons may set up an inflammatory condition, as may also moldy or putrid food.

In birds deprived of grit, the food passes from the gizzard without being triturated and causes severe enteritis with hemorrhage into the lumen of the intestine.

Symptoms. The most prominent symptom of enteritis is diarrhea. The droppings may be watery or semi-solid. The color varies according to the particular disease which is present. It may be white, brownish, greenish, yellowish or mixed in color. The fowl displays a loss of appetite in the more severe forms of enteritis, becomes droopy, weak and somnolent. Paralysis of the legs frequently occurs in connection with inflammations of the intestine. Unusual thirst is often in evidence.

Treatment. As enteritis is usually associated with some more important disease, the disease should be properly diagnosed and treated. Simple enteritis due to minor causes may be treated by remoring the irritating substances from the intestinal tract by means of a purgative such as castor oil in 2 teaspoonful doses or Epsom salts in $1 / 3$ to $\frac{1}{2}$ teaspoonful doses. A tonic consisting of powdered gentian 1 dram, powdered ginger 1 dram and powdered sulphate of iron 15 grains may be given. Mix $1 / 4$ teaspoonful in the feed twice daily. The water for the entire flock may be made antiseptic by the addition of $3 / 4 \mathrm{oz}$. of carbolic acid to the gallon of water. This will tend to prevent the spread of infection to others of the flock in the event that the affected bird or birds are harboring an infectious disease. The carbolic acid treatment may be discontinued after a few days in the event that no more fowls are attacked.

\section{SIMIPLE DIARTHEA}

Fowls are sometimes affected by a diarrhea which is not apparently associated with one of the more important diseases. A watery or semi-fluid discharge may result from an irritation or derangement 
of the intestine independent of the action of infectious organisms. Among the causes which may produce a catarrhal condition of the intestinal mucous membrane and result in simple diarrhea are digestive disturbances of an obscure nature, sudden changes in the feed or an unusually large supply of green feed, too much meat scrap, especially irritating substances in the feed, moldy grains or decomposed flesh. Mineral or other irritants which are not taken in sufficient quantity to cause poisoning or enteritis may produce a simple diarrhea. Fermentation of the contents of the intestine may result in diarrhea.

Treatment. The cause of simple diarrhea being direct intestinal irritation, the obvious remedy is to remove the cause. Where only a few birds are affected they should be given 2 teaspoonfuls of castor oil each. The entire flock may be given Epsom salts if necessary, in the proportion of $1 / 3$ to $1 / 2$ teaspoonful to each grown fowl. The salts may be dissolved in water and mixed in a mash to be fed early in the morning. If suspicion points to the feed as the cause of diarrhea it should be changed or fed in smaller amounts combined with other feeds.

\section{CONSTIPATION}

This is apparently not a common ailment of fowls. It does, however, occur to some extent in fowls confined in small quarters for long periods with insufficient exercise. Lack of green or succulent feed also tends toward constipation. Following diarrhea there may be a temporary or slight constipation. The condition in young chicks suffering from white diarrhea known as "pasting up behind" cannot be considered a true constipation since the droppings are merely held back by the drying of the discharges in the down surrounding the vent. Fowls allowed free range, or large runs and properly fed are practically free of constipation.

Treatment. The condition may be relieved by administering substances which will cause an increased secretion of fluid from the intestinal mucosa, or increased peristalsis. Two teaspoonfuls of castor oil or $1 / 3$ to $1 / 2$ teaspoonful of Epsom salts to each affected bird would be indicated.

Gage and Opperman have determined the dosage of Epsom salts for birds of various ages as follows: 
Age

1 to 5 weeks

5 to 10 weeks

10 to 15 weeks

15 weeks to 6 months

1 year and over
Dose

10 grains

15 grains

20 grains

30 grains

40 to 45 grains

To facilitate figuring quickly the number of ounces required for treating a number of birds they furnish the following information concerning equivalent weights:

1 pound avoirdupois equals 7000 grains.

1 ounce avoirdupois equals 437.5 grains.

1 pound Troy or apothecary equals 5,760 grains.

1 ounce Troy or apothecary equals 480 grains.

The constipation may be due to obstruction by foreign bodies, to worms in the intestines, or to matting of feathers over the cloaca. In the latter condition, softening of the mass and removal of the obstruction is first in order. After removal of the material in the cloaca, rectal injections of soapy water or olive oil may be made. In very small birds a probe wet with glycerine or castor oil may be inserted in the rectum.

\section{DISTENTION OF THE BURSA OF FABRICUS}

Oceasionally the bursa of Fabricus may become enormously distended into a cyst-like structure containing a clear, colorless fluid. Schantyr has observed two cases in which the fluid amounted to over 700 c.c. He observes that when an exploratory puncture is made in case of distention of the abdomen with fluid, a vellowish fluid indicates ascites, while a colorless fluid indicates hydrops of the bursa of Fabricus.

\section{DISORDERS OF THE LIVER}

Disorders of the liver such as enlargement, fatty degeneration, jaundice and the occurrence of various sorts of diseased areas in the organ cannot be classified as separate entities. In most cases, liver lesions merely consist of a portion of the lesions characteristic of the common infectious diseases. Various septicemias, tuberculnsis, entero-hepatitis, leukemia and tumors may display more or less characteristic lesions in the liver. Perhaps under some ennditions hypertrophy or enlargement of the organ may be due to heary 
feeding under close confinement as occurs among fowls fed on corn in the winter.

\section{DISEASES OF THE RESPIRATORY TRACT}

\section{BRONCHITIS}

Fowls are quite frequently affected by a catarrhal inflammation of the mucous membrane lining the air passages of the throat and lungs. This condition usually results from the extension of inflammation from the mouth and nasal chambers. Often birds which have recovered from avian diphtheria are observed to be affected with bronchitis. This may persist for a short time or become chronic.

Symptoms. In severe cases the forvl displays a marked difficulty in breathing. The neck is more or less straightened out to facilitate the passage of air. The breathing is noisy and the fowl may gasp in a manner similar to that shown by a chicken affected with gapes. The beak may be kept open, and a mucous discharge is given off through the mouth. The bird is observed to make efforts to dislodge this discharge by shaking the head.

In less severe or chronic bronchitis the symptoms are not so noticeable. There may be only slight difficulty in breathing, accompanied by a wheezing sound. The mucous membrane of the bronchi and trachea becomes thickened and may show patches of yellowish exudate especially in the two large bronchi.

Treatment. Since bronchitis is often attributed to exposure to cold draughts and dampness these conditions should be corrected. Affected birds should be placed in warm quarters, free from air currents. Under good hygienic surroundings the attack may pass off in a short time. In acute cases the administration of 5 drops of wine of ipecac is beneficial. In chronic bronchitis treatment is not satisfactory.

\section{CONGESTION OF THE LUNGS}

The lungs may become congested as a result of the extension of an inflammation from the larger bronchi into the minute air tubes and air sacs. The congestion is generally of an acute type. It may be induced by the fowl becoming wet and severely chilled. In the early stages of pneumonia congestion of the lungs is the most marked symptom.

Symptoms. Simple congestion is differentiated from pneumonia on post mortem examination by the bright red appearance of the 
lungs in the former and by the fact that pneumonic lungs are hepatized and will sink when placed in water. Affected bixds show a distressed appearance and breathe with diffienlty. The respirations are short and panting. The discharge and wheezing observed in bronchitis are absent. The normal temperature of $107^{\circ}$ to $10 \mathrm{~S}^{\circ} \mathrm{F}$. is raised 2 or 3 degrees. The birds have an increased thirst. Evidence of pain is produced by pressure on the ribs over the lung area.

Where both lungs are affected death may follow shortly after the onset through pressure on the air cells resulting in at shutting off of the air supply and suffocation. In less severe cases the congestion may subside or the condition may develop into pneumonia.

Treatment. The bird should be placed in warm quarters and given a diet consisting largely of green food. One third to one half teaspoonful of Epsom salts dissolved in water should be administered once a day for two or three days. Also give 10 drops of spirits of nitre twice daily.

\section{PNEUMONIA}

Characterization. Pneumonia of fowls is of relatively rare occurrence in the classic form distinctive of this disease in other animals. The disease is sporadic in character and frequently only one bird in a large flock may be infected.

Etiology. Pure pneumonia cases which have come under the observation of Gallagher have been due to the presence of the pneumococcus. It has the characteristic diplococens form on microscopic examination and is similar in its cultural characteristics to the pneumococcus of Frankel. Its infrequent sporadic invasion suggests that the fowl is normally resistant, and that predisposing causes of an obscure nature are responsible for its pathogenicity in certain individuals.

Symptoms. The most characteristic symptoms are more rapid breathing with physical signs of distress and general malaise. There may be a mucous discharge from the nostrils, and the temperature is elevated. As the disease progresses, the comb assumes a darker color at the tips, or rear, or in hanging combs at the more dependent portions. This darkened appearance progresses with the disease and practically the entire comb may assume a purplish color due to lack of oxygen in the circulating blood and weakened heart action. In fatal attacks, the strength fails rapidly and the hird reaches a comatose condition in which it may linger for several hours before death. 
Morbid anatomy. In the normal fowl, on post-mortem examination, the lungs are found deflated, fairly compact and closely applied to the ribs. They occupy less volume relatively, than is the case in other animals. This is, no doubt, due to the peculiar respiratory system of birds which has, in addition to the lungs, a number of air sacs in the pleural and abdominal cavities, and also air canals in the larger bones. In a pneumonic condition the lungs are distended, darkened and solidified to a greater or less degree, approaching the condition known as red hepatization or liver consistency. They will sink in water, while normal or simply congested lungs will float. The pneumonic lung stands out in a firm position surrounding the heart and may be found adhering to the pericardial sac. There are usually no visible areas of necrosis, but the bronchioles and larger air tubes are filled with an emphysematous mucous discharge.

Treatment. Owing to the fact that pneumonia is induced by the presence of organisms in the lung tissues, and that these cannot be reached by disinfectants which would not destroy the sensitive membranes of the air cells, treatment has been found futile in most cases. During the early stages, or in milder cases, stimulants such as strychnine in doses of $1 / 60$ to $1 / 30$ gr., or 50 per cent alcohol in quantities of $1 / 2$ dram may be given by way of the mouth. Because of the dense breast muscles, local external applications are not indicated. The patient should be placed in quarters where the temperature approximates $70^{\circ} \mathrm{F}$. and soft mashes, or sweet or buttermilk given as a food. Since the disease is sporadic in a flock, no special preventive measures are necessary to guard against its spread.

\section{DISEASES OF THE OVIDUCT}

INFLAMMATION OF THE OVIDUCT

The oviduct may be highly congested or inflamed as a result of the condition known as "egg bound," prolapse, or the presence of accumulated egg material which has been arrested by a tumor formation in the wall of the oviduct. Organisms which gain access to the oviduct find a favorable medium for development in such material. They give rise to decomposition products which irritate the mueosa of the organ and set up an inflammatory condition. Broken eggs in the oviduct or excessive effort in laying may lead to inflammation. The oviduct may become affected through the spread of inflammation from the ovary or other adjacent organ or it may be involved with other organs in certain generalized diseases. 
Symptoms. Where the inflammation is confined to the oviduct no general change in the appearance may be apparent except in severe cases. A tendency of the fowl to make efforts to lay at frequent intervals is often noticed. Eggs which are abnormal either in their structure or form may be passed. These may be blood stained. MLst frequently the eggs are small and contain only albumin. Shell-less eggs are also laid. In fatal cases the fowl exhibits symptoms of general debility, with loss of appetite, pale comb and leg paralysis.

Treatment. When symptoms are apparent an effort should be made to locate the cause of the trouble. Manipulation with the finger through the cloaca or over the abdominal wall may disclose the presence of an obstructed egg, broken egg or egg concretion which may be removed after the manner described for the treatment of "egg bounl." The fowl should be isolated, given green food, soft mashes and $1 / 2$ teaspoonful of Epsom salts.

\section{RUPTURE OF THE OVIDUCT}

In severe inflammation of the oviduct when a large mass of egg concretion is present, or an attempt to pass an egg of large size is made, the wall of the oviduct may be split allowing the mass or egg to pass into the peritoneal cavity. Other eggs or egg material mar follow through the opening and accumulate in the carity. These later induce peritonitis, or the rupture may heal and the oviduct continue its normal activities.

The condition would be suspected by the sudden suspension of laying accompanied in many cases by a rapid increase in the size of the abdomen. Manipulation would disclose the presence of abdominal egg concretions or accumulated eggs.

Rupture of the oviduct is of comparatively rare occurrence. Treatment if advisable would be by surgical means.

\section{EGG BOUND}

This condition is of frequent occurrence in fowls, especially in rommg pullets. It represents an inability on the part of the bird to pass the egg in the normal manner from the oviduct, or cloaca. This failure may be due to an inflammatory condition, stricture, or tumor formation in the posterior portion of the oviduct, or cluaca, or to prolapse of the oviduct. In pullets which are beginning to lay, howerer, the usual cause is the undeveloped state of the egg. 
passage which has not yet become sufficiently dilated to accommodate eggs of large size. Mature hens may become egg bound through attempts to pass malformed, or double yolked eggs. It is generally noted that the first eggs of the pullet are elongated and of smaller diameter than those delivered after laying has become well established. Also the first eggs frequently show streaks of blood indicating the difficult passage through the last portion of the oviduct or through the vent. Cross breeding which unites a breed of large egg type with one of a smaller egg type would be conducive to the formation of a large egg for which the egg passage might not be developed in proportion.

Symptoms. The affected hen is observed to be restless, leaving the others and going frequently to the nest to make attempts to lay. The effort after a time results in an inflamed condition of the oviduct and cloaca. The condition often results in an eversion or prolapse of these organs. The distress of the fowl and the extruded, inflamed parts may attract other fowls. These begin to pick at the membranes and if the victim is not rescued in time a large portion of the intestine may be torn away and dragged through the vent causing the death of the bird. Where prolapse has not occurred the affected fowl may continue to make an effort to lay the egg until successful or overeome by weakness.

Diagnosis. The presence of an egg in the oviduct may be determined by palpation of the posterior abdomen or exploration through the vent.

Treatment. Several methods of treatment are practiced. The fowl may be held vent downward over steaming water for a time and then placed upon the nest. The steam has a tendency to relax the parts and make easier the voiding of the egg. Lubricating the vent and cloaca with sweet or linseed oil may also aid the fowl in mild cases of egg bound. However, the quickest and surest method is the removal of the egg by the following procedure. The hen is held by an assistant with her back downward, while the operator passes the forefinger through the vent and pushes aside the membranes until the egg shell is felt. With the fingers of the other hand pressing on the external wall of the abdomen the egg is forced toward the vent, being guided along the inflamed membranes of the egg passage by the inserted finger. When the shell is visible through the vent it is punctured by means of a sharp pointed instrument such as a knife or awl. It is broken into small pieces with a pair of forceps or with the finger and is removed with its contents. The 
patient should be isolated and the inflammation redueed by frequent injections of cold or ice water into the cloaca.

Fowls which have suffered from egg bound due to the presence of a large egg or to tardy development of the egg passage are not permanently affected. Egg bound due to pathologic alterations in the oviduct is not open to practical treatment.

\section{PROLAPSE OF THE OVIDUCT}

Eversion of the oviduct is usually associated with difficulty in laying as in the condition known as "egg bound." The mucosa of the extruded cloaca and oviduct becomes highly congested. The prolapsed organs are observed as a dark red or purplish mass projecting through the vent. The affected forvl is soon noticed by the others of the flock and if it is not rescued in time it will be attacked and destroyed as a result of the protruding tissue being torn away and devoured.

As soon as the bird is discovered it should be placed by itself and carefully treated. The oviduct should be explored with the finger, which should be greased or oiled, to locate an egg or other cause. If an egg is present it may be removed as described for "egg bound." The prolapsed tissue is carefully returned through the vent, after which cold water is injected to reduce the congestion and promote the contraction of the oviduct wall. This may be continued for some time or repeated at frequent intervals until a cure has been effected.

\section{VENT GLEET}

Characterization. Tent gleet or eloacitis is an inflammatory disease of the vent and cloaca of fowls. It appears to be spread entirely by coitus and may be considered an infections venereal disease. Its causative agent has not been determined.

Symptoms. In the early stages of the disease there is a congestion of the membrane of the posterior portion of the cloaca and margin of the rent. This may extend to the lower portion of the oriduet and rectum. The fowl exhibits signs of severe irritation, frequently voiding small droppings. A watery discharge which later beenmes purulent and foul smelling is passed out through the vent. The skin around the rent beenmes swollen and reddened and an offensive odor is usually present. The irritation causes the fowl to peck at the part. Other fowls may be attracted by the discharge, and the reddened appearance of the affected region and may peek at the skin 
causing an ulceration of the surface. Frequently the diseased fowl dies as a result of the tearing of the cloaca and rectum by its cannibalistic mates. The presence of the disease in a flock causes a marked falling off in the egg yield and a decrease in the fertility of the eggs.

Treatment. Vent gleet is very resistant to treatment. It is usually better to destroy the diseased bird than to attempt treatment. This is especially so in the case of the first birds attacked as by this means its spread may be prevented. Where treatment is desired the affected birds should be isolated. The discharge may be removed from the skin and feathers by washing with warm water. Antisepties such as 5 per cent carbolic acid, or 1-1000 corrosive sublimate are to be applied to the external affected area, or a 2 per cent carbolic ointment may be rubbed over the part. Argyrol in 15 per cent solution or cresol in 2 per cent solution may be injected into the cloaca twice daily. Roosters should be removed from the flock while the disease is present and if any are found diseased it would be advisable to destroy them.

STRUCTURE OF THE FECUNDATED EGG OF THE FOWL

The ovum or yolk on its release from the ovary and entrance to the oviduct appears as a yellowish ellipsoid body about one inch in diame-

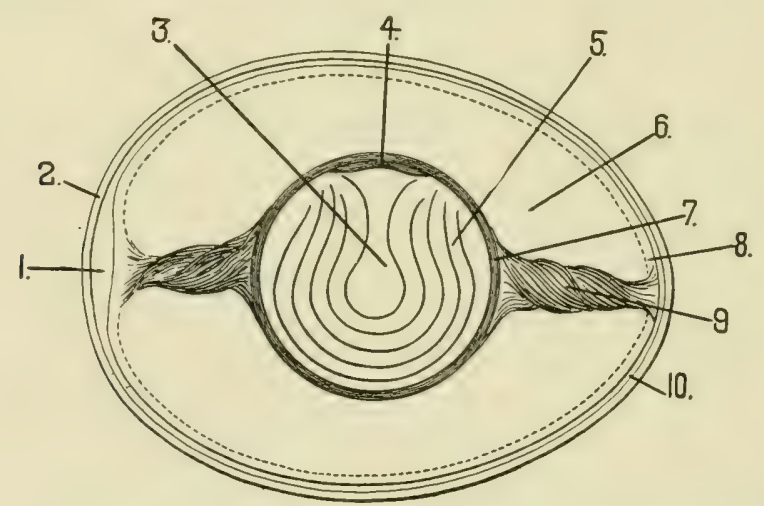

FIG. 18. 1, air sae; 2, shell; 3 , white yolk; 4, blastoderm; 5 , yellow yolk; 6 , dense albumin; 7 , chalaziferous layer of albumin; 8 , fluid albumin; 9 , chalaza; 10 , shell membrane. (Original)

ter. It is somewhat flattened on one surface, which presents an opaque whitish circular spot, the germinal dise (Fig. 18). Cell 
multiplication is already taking place in this dise, from which the embryo develops. The yolk is composed of a central light colored portion (Fig. 18) and an outer mass colored a deeper yellow. The latter is formed of concentric layers showing different stages of yolk deposit (Fig. 18). The germinal dise and yolk are surrounded by a thin strong vitelline membrane (Fig. 18). The ovarian ovum on entering the oviduct is passed along by peristaltic movement in a rotary course to its completion in the uterus or shell secreting portion. In the anterior portion of the oviduct the membrane chalazifera is applied over the vitelline membrane. It consists of a dense layer of albumin and gives rise to the two chalazæ which extend from each pole of the ovum. These strands become twisted in opposite direction during the further passage of the ovum through the oviduct. Over the chalaziferous membrane are several albuminous layers secreted by the glands of the oviduct. When the ovum enters the isthmus or narrow portion two egg membranes are secreted. These become separated at the large end of the egg as air is absorbed. The shell is acquired in the uterus.

\section{FOREIGN BODIES IN EGGS}

Eggs may contain a variety of foreign bodies such as pebbles, fragments of plants, feathers, roundworms, etc. Such bodies after gaining access to the cloaca penetrate the oviduct far enough to encounter an egg and thus be included within the shell.

Otherwise normal eggs have been found to contain such parasites as the fluke Prosthogonimus ovatus and the roundworm Ascaridia perspicillum. The latter worm may be present surrounded by albumin in an abnormal egg lacking a yolk. It is apparent that under such circumstances the presence of the worm has stimulated the secretion of the albumin. Tapeworms and segments of the same have been found in eggs. The parasites found in eggs evidently have migrated into the oviduct for a sufficient distance to meet a developing eg. and to become enclosed in shell. Parasites of microscopic size such as coccidia, amebæ, aspergillus fungi and bacteria have been demonstrated in eggs.

\section{BLOOD SPOTS IN EGGS. (LIVER SPOTS)}

It frequently happens that streaks of blood, or clots of varying size are found in eggs. This abnormality is especially noticed during the heavy laying period. At such time the ovary is plentifully supplied 
with blood to promote the rapid formation of ova. As an ovarian follicle ruptures to release an ovum some blood may escape from a congested blood vessel of the follicle. The blood passes into the oviduct aleng with the ovum where it becomes surrounded by albumin and is incorporated in the egg. The size of the spot or clot depends upon the amount of blood which has escaped. These clots are often taken for pieces of flesh and are commonly referred to as liver or meat spots. The wholesomeness of such an egg is not impaired. There is no practicable means known for preventing the occurrence of blood spots in eggs. However by candling the entire product of a flock, it is possible to prevent such eggs frem going on the market.

\section{DISCOLORED YOLKS}

Newly laid eggs in some instances exhibit a darkened appearance of the yolk which is not due to developmental changes in the ovum or to decomposition as a result of bacterial action. The entire yolk may appear dark in color or the discoloration may be confined to spots or streaks on the surface or in the depths of the yolk material. In testing for freshness by candling or on opening the egge, such a discoloration may be mistaken for an evidence of addling. The condition results apparently from the action of sulphur, normally present in the yolk, on small globules of cottonseed oil which are absorbed into the yolk. This occurs when the fowls are being fed cottonseed meal or material containing cottonseed oil. This oil may be present in table scraps since it is often an ingredient of butter, oleomargarin or cooking oils. Cattle or hog fats or their products may contain cotton seed oil where the animals have been fed cotton seed meal shortly before slaughter.

Yolk is composed of albumin 17.50 per cent, oil and salts 28.75 per cent, and water 53.75 per cent. The yolk granules are arranged in thin concentric layers as they are received through the surrounding vitelline membrane from the blood vessels in the ovarian capsule or follicle. This arrangement would explain the disposition of the discoloration, depending on whether the fowl was receiving cottonseed oil constantly or only at intervals. The color reaction between sulphur and cottonseed oil is utilized in the Halphen test for the presence of the latter as an adulterant in certain food products. In a positive test a pink color is produced but this is changed in the yolk to a brownish or dark yellowish color. It is not improbable that discolored yolks may result also from the 
action of the contained sulphur on certain metallic salts which the fowl may ingest and which through metabolism may be incorporated into a yolk.

There is no apparent reason why egg's so affected should be unwholesome. They may be detected by candling.

\section{DOUBLE YOLKED EGGS}

The inclusion of two yolks within one shell is due to rapid ovulation or delay of one yolk in its progress to the isthmus or egg membrane-secreting part of the oviduct, thus allowing the following yolk to reach it and be surrounded by a common membrane. When the two yolks do not meet until arrival at the isthmus, each is surrounded by a separate layer of albumin. When two yolks are ovulated within a short interval or meet in the anterior portion of the oviduct they may have a common layer of albumin and common membranes.

\section{SOF' SHELLED EGGS}

When the egg reaches the uterine portion of the oviduct under normal conditions the double membrane secreted by the isthmus is covered by a felty fibrous layer into which is deposited the material which forms the calcareous shell. Absence of the hard shell may be due to several causes. If the bird does not have feed containing lime salts in sufficient quantity, or access to substances rich in these salts it is apparent that a normal shell cannot be produced. Providing the fowls with erushed oyster shells or mortar will remedy this defect. Irritation of the oviduct as a result of inflammation may cause an abortion of the egg before it is properly formed. Sudden fright may also lead to a premature laying of the egg before it has acquired its hard shell. Excessive attention by the male is said to cause the laying of soft-shelled eggs. Where this is considered as the cause the removal of the cock is indicated. Except when eggs are desired for incubation the presence of a male bird in the flock is not necessary since fowls lay equally well or better in his absence. Unfertilized eggs keep better than fertilized ones, a fact which causes many poultry men to place cock birds in the flock during the breeding season only. 


\section{DISEASES OF THE PERITONEAL CAVITY}

\section{ABDOMINAL YOLK CONCRETIONS}

The presence of masses of yolk material lying free in the peritoneal cavity of hens is frequently observed on post-mortem examination. The cause of this condition is not always apparent. Injuries to the membrane of the fallopian tube, or tumor formations in the oviduct which interfere with the entrance of the volk or its passage through the oviduct are usually responsible. Yolks or partly formed eggs may be returned through the oviduct and aborted into the peritoneal cavity by reverse peristalsis induced by inflammatory changes in the wall of the oviduct. At times the contents of only one yolk or orum may be present while in other cases the concentric layers of yolk substance indicate that the process has been going on for a considerable period of time. In the latter instance the mass may be as large as a tennis or base ball and of firm consistency. The layers are easily separated when newly formed or before organization or decomposition changes have progressed. Some accumulations reveal a center of albumin, or partially formed shell membrane, while others exhibit a yolk formation throughout. Occasionally a normal yolk is found surrounded by a small amount of albumin and inclosed in a soft or partially calcified membrane. In this case there is evidence that the ovum has passed into the oviduct and owing to an abnormal state of this organ, reverse peristalsis has caused its return through the fallopian membrane into the abdominal cavity. Quite frequently as many as five to seven perfectly formed yolks in a good state of preservation are found between the intestinal convolutions. These apparently represent a recent discharge from the ovary in contrast to the solidified yolk substance constituting the typical yolk concretion.

Symptoms. The presence of yolk deposits is not disclosed by external appearances where putrefactive changes are absent. The tendency is for the material to become partially absorbed. The unabsorbed portion is formed into a rounded body which increases in size according to the number of ova aborted into the cavity. In the event that organisms find their way into the peritoneal cavity, presumably by way of the oviduct, a favorable culture medium is at hand and decomposition changes may be inaugurated which lead to a septic condition and result in peritonitis, or septicemia. 
Treatment. The removal of the yolk masses is easily accomplished by an incision through the abdominal wall once the nature of the affection has been determined by digital exploration. As a rule the presence of aborted egg material is not suspected during the life of the fowl and treatment is therefore not practiced. It is also apparent that treatment to be effective would require the correction of the factors which lead to the misplacement of the ova. The obscure nature of these renders attempts at their control impractical.

\section{DROOPING ABDOMEN}

In the heavy breeds, especially, the abdomen may show a tendency to assume a pendant position, frequently coming into contact with the ground when the bird is in a standing position. The skin of the abdomen may be devoid of feathers, and usually shows a reddened appearance. This is often due to a marked accumulation of fat in the abdominal wall and visceral organs. Nore often it results from a displacement of the gizzard. This organ, owing to an elongation of the proventriculus or true stomach, is carried backward and instead of resting in its normal position on the posterior floor of the sternum, takes up a position on the much less resistant membranous wall of the abdomen. As a result of its weight and muscular activity in a cavity already overfilled with distended intestines and accumulated fat it forces the inferior abdominal wall into a drooping or dragging position. The abnormal position of the gizzard may be detected by palpation of the parts immediately behind the sternum where the outline of the dense muscular organ can easily be followed.

Treatment consists of the restriction of fat forming foods in the diet. It is inadvisable to use birds showing this condition for breeding purposes, since the evident tendency to lack of tone and resistance in the parts concerned would be perpetuated to a greater or less extent in the progeny.

\section{ASCITES (DROPSY)}

Characterization. This condition consists of an accumulation of fluid in the peritoneal cavity or abdomen.

Etiology. No one specific causative agent is responsible. The accumulation of fluid results from a filtration of blood scrum through the serous membranes of the intestine, or the peritoneal covering of the abdominal cavity or paryenchymatous organs. It 
may be present in severe, or chronic cases of enteritis, sarcomatosis, tuberculosis, peritonitis or other debilitating diseases affecting the abdominal organs.

Symptoms. Except in a marked dropsical condition, no external manifestations of ascites are readily noticeable. On palpation of the abdominal region the presence of fluid may be detected. Its presence has no great diagnostic importance since it is merely associated with a more serious affection which may be difficult of accurate determination during the life of the bird and which in the large percentage of cases is not open to practical treatment.

Differential diagnosis. Ascites is often confused with an excessive formation of fat in the abdominal wall or with a drooping abdomen. Palpation of the abdomen and the absence of emaciation or other signs of disease would eliminate ascites. When the latter is present a distinct fluctuation of liquid is felt, much the same as in the palpation of a water bag.

Treatment. When detected, the fluid may be evacuated by puneture of the abdominal wall with a hypodermic needle. Except in cases of septic ascites this method of procedure would be of little permanent value since the fluid would be readily absorbed in the event that the primary cause of its presence were corrected.

\section{PERITONITIS}

Characterization. Peritonitis occurs frequently in domesticated birds. It is manifested by an inflammatory condition of the serous coverings of the visceral organs accompanied by a serous, or coagulated exudate in the peritoneal cavity.

Etiology. Various causes may be responsible for this affection. There are numerous specific diseases of a septicemic nature in which the involvement of the peritoneum is of secondary consideration. Besides these the most important direct causes of peritonitis are infected rolk concretions or deposits, ovarian infections, extension of inflammation of the oviduct, or rectum, as a result of rupture, perforation of the digestive tract by foreign bodies or as a result of ulcerative changes in the intestinal wall such as sometimes occur in coccidiosis of fowls, or entero-hepatitis of turkeys.

Symptoms. These are nonspecific since the general attitude of an affected bird is similar to that displayed in several other infectious diseases. Diagnosis is difficult except through post-mortem examination. The disease is usually of an acute nature, death 
resulting shortly after the appearanee of symptoms. The affected fowl shows loss of appetite, ruffled feathers, elevation of temperature, weakness and extreme depression. Diarrhea is frequently observed especially when the intestinal serosa is extensively involved. In cases of peritonitis due to rupture of the rectum, the droppings are scanty owing to the passage of a portion of the intestinal contents into the peritoneal cavity. The presence of yolk concretions, or fluid in the abdominal cavity may assist in a diagnosis. In either case the abdomen may show a pendant position. On palpation, the fluctuation of liquid may be felt, while the presence of enneretions is determined by their dense consisteney, free disposition and rounded contour.

Morbid anatomy. The pathologic picture in peritonitis in birds is not usually marked by extensive congestion of the serous lining of the abdominal cavity and visceral organs. There may be localized areas of congestion, or hemorrhagic spots on the visceral peritonemm, especially that covering the ovary. In the majority of cases no macroscopic evidence of congestion is apparent. The disease is more often marked by exudates either of a serous, or semi-solid form. Evidently the morbid condition results principally from the absorption of toxins generated by the action of microorganisms on foreign matter which has gained entrance to the cavity. The serous exudate is at times clear and straw colored, at other times cloudy and putrid. The septic condition is generally associated with a cheesy exudate which covers the viscera in small masses and is non-adherent to the membrane. Yolk concretions, or individual aborted yolks, display putrefactive changes. Where rupture of the oriduct or intestine has cccurred, egg material or intestinal contents respectively will be found and the rupture may be easily located.

Treatment. The difficulty in diagnosing peritonitis during the life of the bird and the advanced stage reached when srmptoms become apparent renders attempts at treatment impractical. Should a physical examination reveal the presence of yolk masses free in the abdominal cavity, or a dropsical condition of the abdomen, surecical methods could be employed to remove the acemulations. The cavity eould then be washed with sterile water or a mild antiseptic to remove septic exudates.

\section{VICIOUS IIABITS}

Toe pecking. The conditions surrounding incubator chickis kept together in large mumbers in brooders, are very different from those 
afforded a brood of chicks under the care of a hen. One of the most troublesome results is the habit of pecking toes. Anything unusual about the foot of a chick attracts the attention of another member of the flock which pecks at the foot. The attention of others is drawn to the action and they repeat the pecking. As soon as blood is drawn, the deep seated instinct of chickens to attack the injured or the weakling, is aroused and the victim is soon killed or seriously maimed. The habit is likewise manifested by pecking at the vent. In this way a chicken is disembowled in a surprisingly short length of time. Once the taste for blood is aroused, the chicks attack one another indiscriminately and cause heavy losses.

The immediate remedy is to remove instantly all wounded birds, which must be kept in isolation until entirely recovered. General precautionary measures will include those designed to keep the chicks occupied with harmless pursuits. Various kinds of food hung so as to be somewhat inaccessible, furnish diversion. Dried meat has been so employed.

Egg eating. The habit of eating eggs is often established by the accidental breaking of an egg. The practice spreads rapidly by imitation. Careful observation will reveal yolk stains on the beaks of offenders, who should be isolated or killed.

Nests well supplied with straw will minimize the possibility of the accidental breaking of eggs, and darkened nests will prevent a broken egg from being seen. Craving for lime in the egg shells may be forestalled by supplying an abundance of oyster shells and bone.

Feather eating and feather pulling. These habits constitute very troublesome vices not only among fowls but also in cage birds. A bird may pull out its own feathers or those of its companions. The sensation of having the feathers plucked apparently is not disagreeable, for a bird will stand quietly while another pulls out its feathers. Finally the birds taste blood on the emerging feather and as a result, never allow a feather to develop. The vice is often observed in cage birds such as parrots, among hens that are closely confined, and occasionally among hens lacking certain elements in the feed. Parasites of the skin are important causes of the itching leading to feather pulling. In some instances the eruption of normal feathers during molting, occasions an itching sensation and starts the habit. Perhaps the most common cause is a monotonous diet of a restricted number of elements. Hens at large in warm weather can satisfy individual food requirements from among the 
various materials available, such as grass, insects and mineral substances.

Krlee finds it very useful to supply blood in a cooked form, mixed with bran and eurd. Under these conditions the birds do not aequire a taste for blood. Burned shells and green feed are also desirable elements in the ration. Green feed is best surrounded by a wire netting in such a way that the birds are compelled to reach through the meshes to obtain the feed. In winter time cooked beets or sprouted oats may be used.

Breaking up the habit in parrots often causes difficulty. Daily sprinkling with water and providing variety of feed are useful. Klee also has observed many cases benefited by smearing the feathers with sepia, or unsweetened chocolate and by keeping the bird in the dark. It is possible to fit a broad aluminum collar on the neck of a parrot in such a way as to prevent the bird from reaching the feathers.

According to Klee, lack of feathers is observed in pigeons from time to time without being due to feather pulling. In some instances the pin feathers are present in the skin but do not break through. Persistence of the feather sheath also occurs in pigeons, in which case the feathers develop while rolled up in a horny covering. Under normal conditions this sheath is shed early in the development of the feather. Sometimes if the bird is kept until the molting period the new feathers will develop normally.

In chickens and in any growing birds, interference with nutrition such as intestinal catarrh will cause retardation of the feather development in the molting period. Molting exerts such a drain upon the strength of birds that only a well nourished bird is able to molt normally. As a result, birds are more inclined to become diseased during molting than afterwards.

\section{REFERENCES}

1. Dickson. Botulism. A cause of limberneck in chickens. $J .-A m$. Vet. Med. Ass., Vol. 3, 1917, p. 612.

2. Hebrant et Antoine. $\Lambda$ propos de la goutte ou diathèse urique. Ann. de Méd. Vét., T. 58, 1909, p. 321.

3. Kilee. Die hauptsächlichsten Geflugel-Krankheiten. Leipsig. 1905. 


\section{CHAPTER XVI}

INTERNAL PARASITES

pARASITES OF THE ESOPHAGUS, CROP, PROVENTRICULUS AND GIZZARD

FOWLS

Dispharagus nasutus, a filarial worm 7 to $9 \mathrm{~mm}$. long, is one of the most important parasites occurring in the portion of the alimentary tract of fowls anterior to the gizzard. The worms are found in great numbers in the mucosa of the proventriculus and of the gizzard. The affected birds become emaciated and depressed without losing appetite. The mucosa of the gizzard has been observed to be bristling with the parasites, some in the mucosa and others attached to the mucosa by one end and floating free in the lumen. The proventriculus when infested may be increased to double the usual size, becoming spherical in form. The mucosa is greatly thickened in places where the parasites are most numerous. Cases have been observed in which the worms were so numerous as

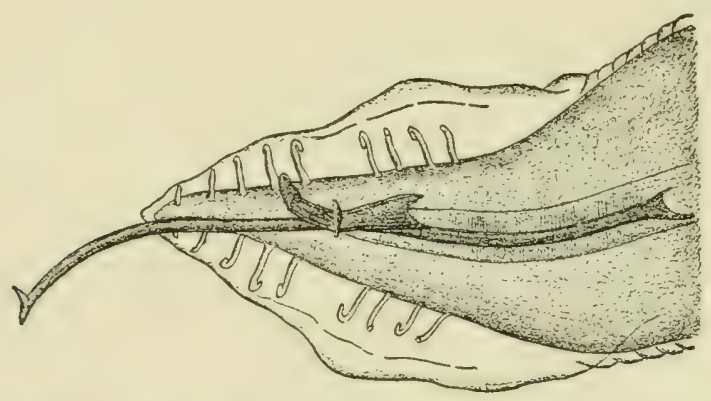

Fig. 19. Spiroptera pectinifera. Male. Caudal extremity. $\times 175$. Neumann)

to give the glands a villous appearance. Colucci obtained good results by the administration of capsules of .5 gram of oil of turpentine morning and evening.

D. hamulosus has been reported from fowls in Brazil as occurring in little fleshy excrescences on the surface of the gizzard. D. laticeps has been found in the fowl and certain rapacious birds. Physaloptera truncata has been observed in the gizzard of a fowl in Brazil. spiroptera pectinifera is a hair worm which infests the gizzard of 
fowls. Individuals vary from 4 to $9.5 \mathrm{~mm}$. in length and are 2 $\mathrm{mm}$. broad. The worms are attached to the mueosa of the gizzard by their anterior extremities. The mucosa is covered by a brownish deposit 2 to $3 \mathrm{~mm}$. thick, consisting of a mixture of blood and debris of the parasites. The affected mucosa shows irregular depressions which are gramulated, rediened and hemorrhagic. The affected hirds show anemia, progressive cachexia and good appetite to the last. One to three months may elapse between appearance of symptoms and death, depending upon the age and vigor of the subject. This parasite has been reported as eausing heavy mortality among chickens in France. Its presence in the guinea-fowl has been reported.

Ransom has described a nematode worm Gonglyonema inglucicolu parasitic in the crop of fowls. The presence of the females may be detected by noting the cccurrence of small coiled ridges on the surface of the mucosa, discernible with the naked eye. The males are only discovered by the use of a hand lens. Specimens may be obtained for detailed study by teasing the tissue. The females vary from 32 to $45 \mathrm{~mm}$. in length, while the males are from 17 to $19 \mathrm{~mm}$. long. The infestation does not so far as known, result in serious disturbance of health. The same parasite has been recognized in Europe by Ciurea.

Trichosoma annulatum is a worm 15 to $80 \mathrm{~mm}$. in length which has been reported as occurring under the epithelium of the mucosa of the esophagus of the fowl. Another member of this genus, T. nodularis causes serious trouble in waterfowl.

Dispharagus spiralis, a worm 7 to $9 \mathrm{~mm}$. long has been reported in the same location as well as in the gizzard. Prosthogonimus pellucidus, a trematode, has been found in the esophagus of fowls.

\section{PHEASANTS}

Trichosoma strumosum causes serious loss among young pheasants. It lives in the large folds of the epithelium of the esophagus and sometimes penetrates the mucosa. It forms passages in which the female deposits eggs. This destruction of epithelium occurs in the esophagus, buccal eavity and the trachea. The disease causes great weakness at the begimning,

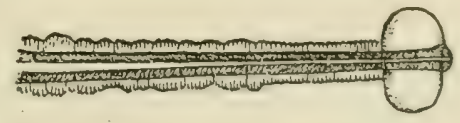

FIG. 20. Trichosoma strumosum. Female. Anterior extremity. (Reibisch) followed by rapid emaciation. Death 
occurs four to six days after the appearance of the first symptoms. Treatment consists of administering a 2.5 per cent solution of carbolic acid in the drinking water or in the mash.

\section{WATERFOWL}

Strongylus nodularis has been reported as occurring in waterfowls, most frequently in the mucosa of the gizzard. The female worms vary in length from 12 to $22 \mathrm{~mm}$. while the males vary from 10 to $16 \mathrm{~mm}$. The parasites are about $5 \mathrm{~mm}$. in diameter, and are threadlike in form. They are more narrow at the ends, especially at the head end. Freese reports a loss of 200 out of 700 geese, due to the parasite.

Symptoms. Single individuals occur very frequently in healthy geese without causing symptoms or noteworthy lesions. Extensive invasion of young geese is attended with serious results. At the beginning of sickness the young geese appear weak, sit most of the time and move with reluctance. They make characteristic choking movements and soon show more or less diarrhea. The birds have a very good appetite and consume their feed ravenously. In spite of the large amount of food consumed, the birds gradually become emaciated almost to skeletons. Finally they fall from weakness and are unable to rise again. In this stage the birds stop eating and die in a day or so. The whole course of sickness occupies three to eight days. The younger the geese, the more they are affected. The young, as a rule, die.

Morbid anatomy. The carcass is excessively emaciated and only traces of the breast muscles remain. The mucosa of the small intestiue, especially near the gizzard, is reddened, thickened and covered with a large amount of viscid mucus. The principal lesions are found in the gizzard. Except where the epithelium normally forms horny plates, it is covered with a thick, bark-like mass, dark brown or dark brownish red in color. The consistency of the mass is more or less tongh and in some places, is more like mucus. In and under this material are innumerable parasites in coils. Not infrequently the worms are attached to the bark-like mass by their heads, with the other ends free in the lumen. Sometimes a mucous layer, with worms, is located in the lower third of the esophagus. Occasionally a few worms may be found on the mucosa of the small intestine. The statement of some authors that the parasites occur under the mucosa is incorrect, for they occur only under the epithelium and less commonly directly in the epithelium. 
Dispharagus uncinatus has been reported as occurring in the esophagus and proventriculus of geese, ducks and swans. They oecupy cyst-like structures which are most frequently encountered in the wall

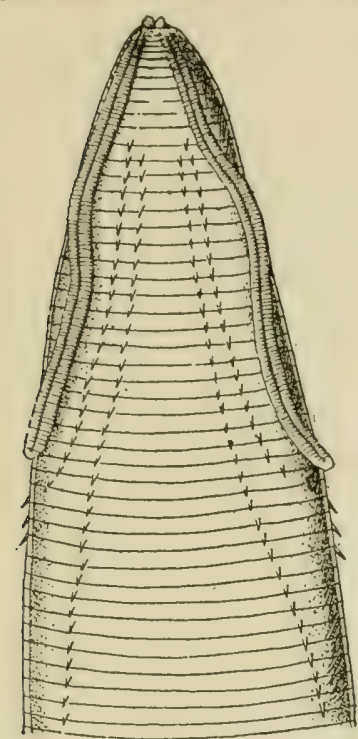

FIG. 21. Dispharagus uncinatus. Dorsa 1 view of anterior extremity. Enlarged 60 times. (Schneider)

of the proventriculus. When these are opened the worms are found surrounded by a bloody fluid or a yellowish caseous mass. The wall of the proventriculus is thickened and the mucosa is covered by a large amount of yellowish green viscid mucus. $\Lambda$ stage of the life cycle of the parasite is passed in a certain aquatic form, Daphnia pulex. The embryos, free in the stomach of the bird, gain access to the water where they are taken in by the Daphnia, which creature is in turn eaten by the waterfowl.

Prophylaxis must depend chiefly upon killing of infected birds, and upon keeping birds away from stagnant water, colored brownish or yellowish by the presence of Daphnia in large numbers.

Sclerostomum anseris infests the gullet, proventriculus and gizzard of geese. It burrows under the mucous membrane and causes the formation of dark brown crustlike layers on the epithelium. In young ducks it causes gradual emaciation and debility. The location of the parasites discourages the use of antihelminthics.

Hystrichis tricolor is a worm which infests the proventriculus of ducks, and causes extensive destruction of tissue. Very large ulcers are formed which almost perforate the walls of the organ. Cysts about the size of a bean, containing the parasite, are also found. A similar parasite Hystrichis elegans also occurs in the proventriculus of ducks and of a large number of palmipeds. $H$. cygni occurs in similar location in the swan.

Tropisurus fissipinus form cysts in the submucous region of the esophagus of ducks, some-

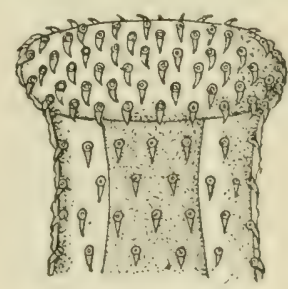

FIG. 22. Hystrichis tricolor Cephalic extremity. (v. Rátz) times causing a fatal inflammation.

Trichosoma contortum necurs in ducks and geese besides other birds. They infest the dilated portion of the gullet, which in dueks 


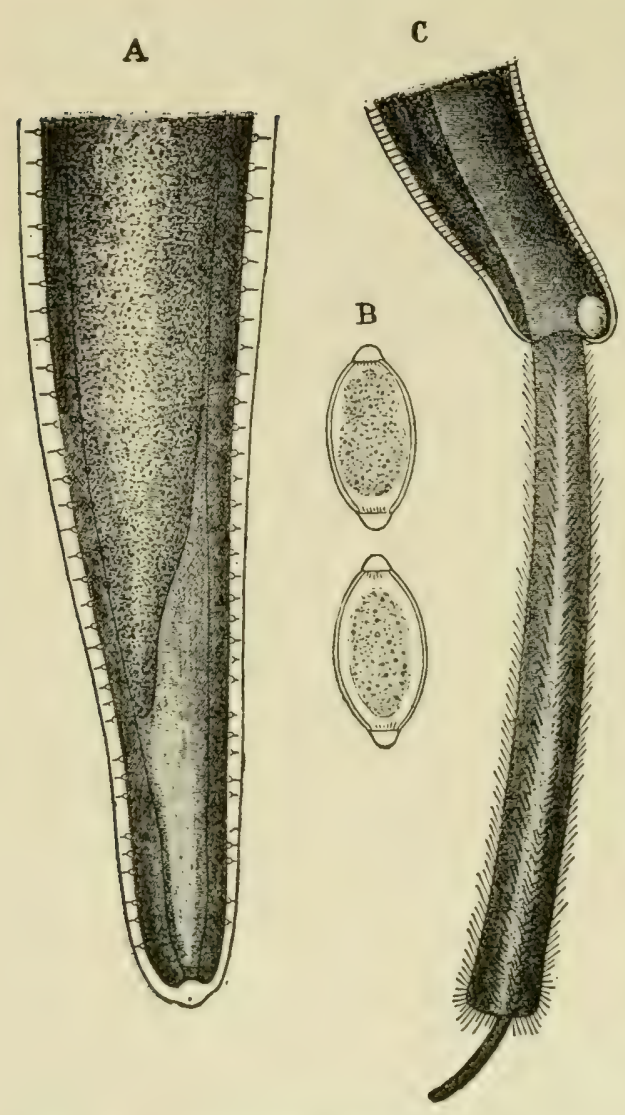

Fig. 23. Trichosoma contortum. A, caudal extremity of female; $B$, eggs from the uterus in various stages; $C$, caudal extremity of the male. Enlarged 300 diameters. (Railliet) and geese, performs the functions of a crop. The presence of the parasites causes a distention of the gullet and a condition quite similar to distention of the crop in fowls. The infested birds display arrested development, emaciation, difficult locomotion, epileptic movements, somnolence and lack of appetite. Death is frequently due to asphyxia resulting from over distention of the gullet.

At autopsy it is observed that the galleries formed by the worms appear as whitish curved lines which stand out in prominent relief against the congested mucosa. Microseopic examination of the tissue will reveal the presence in the galleries of the worms and their eggs.

Positive diagnosis is difficult during life unless microscopic examination is made of the feces or of water with which the crop has been rinsed.

Treatment may consist of administering two tablespoonfuls of a mixture of turpentine with twice its volume of olive oil.

Filaria cygni has been observed in the intestine and ceca of a swan which was exceedingly emaciated.

\section{TAPEWORMS}

General character. Tapeworms, also designated cestodes, constitute important parasites of the intestines of birds. When present in large numbers they occasion a disease designated tæniasis. The individual worm is composed of a head called the scolex and a 
variable number of segments designated proglottides. The scolex is armed with a series of hooks by which it becomes attached to the intestinal mucosa of the host. Each proglottid or segment of the worm is virtually an individual and is bisexual. The segments are not provided with an alimentary canal, but

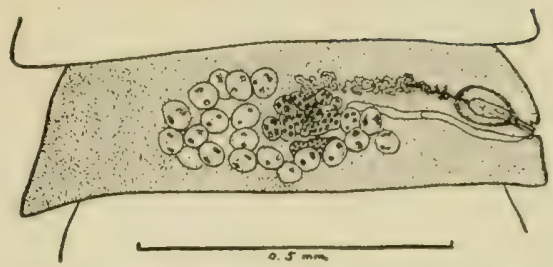

FIG, 24. Segment of Davainea echinobothrida showing the reproductive organs. Enlarged. (Ransom) absorb nourishment directly from the intestinal contents of the liost. As the terminal segments in turn mature, they are separated from the worm and are discharged in the droppings.

Species of cestodes. A large number of species of tapeworms have been reported as occurring in the intestines of domesticated anỏ wild birds. Ransom has examined nine species in American chickens and turkeys as follows: In chickens, Hymenolepis carioca, Choanotaenia infundibulum, Davainea tetragona, D. echinobothrida, D. proglottina, Amoebotaenia sphenoides; in chickens and turkers, D. cesticillus, Hymenolepis cantaniana; in turkeys, Metroliasthes lucida. He notes that the tapeworms of American ducks, geese, and pigeons have not yet been investigated. Neumann lists four more species as having been found in fowls. These are Cotugnia diagonopora, Taenia fasciolaris, T. exilis and Bothriotaenia longicollis.

Tapeworms found in the various birds are listed by Neumamn as follows :

Turkey: Choanotaenia infundibutum, Metrotiasthes Iucida, Davainea cantaniana, D. cesticillus, D. friedbergeri, Hymenolepis carioca, H. musculosa, H. meleagris, Not named.

Pheasant: Choanotaenia infundibulum, Davainea echinobothrida, D. cantaniana, D. friedbergeri, Hymenolepis phasianina.

Pigeon: Choanotaenia infundibutum, Davainea echinobothrida, D. crassula, Bertiella delafondi.

Duck: Hymenolepisanatina, H. gracilis, H. simuosa, H. coromula, H. parvula, H. megalops, H. lanceolata, Choanotaenia infuntibulum, Davainea crassula, Fimbriaria fasciolaris.

Goose: Hymenolepis lanceolata, $H$. setigera, $H$. gracilis, $H$. fasciata, H. sinuosa, H. tenuirostris, Fimbriaria fasciolaris.

Neumann considers tapeworms as oceuring infrequently in pioeons and rarely present in sufficient number to cause trouble. In ducks they exert feeble pathogenic action. IIymenolepis setigera and 
H. lanceotata, singly or together cause injury among geese in Europe. Davainea friedbergeri causes great losses among pheasants.

Life history. From time to time the tapeworm in the intestine of the chicken discharges ripe segments filled with eggs, which are voided in the feces. To maintain the life cycle it is necessary that the tapeworm eggs be taken with the food into some creature known as a secondary host. In this host the embryos escape from the eggs and migrate to some organ where they form a cyst-like structure known as a cysticercoid. The life cycle is completed in case the intermediate host is eaten by a chicken in which event the cysticereoid develops into the adult form.

Comparatively little is known about the secondary hosts of tapeworms of poultry. Of the five species of tapeworms that have been reported in chickens in the United States, the intermediate host of but one has been discovered. It has been determined that the intermediate host of Choanotania infundibulum is the common house fly, Musca domestica.

The intermediate hosts of a number of tapeworms of birds reported as occurring in other countries have been discovered. 'Thus a slug is necessary for the life cycle of Davainea proglottina, a chicken tapeworm. The intermediate hosts of certain tapeworms of the duck have been found to be various species of fresh water crustaceans.

In general, the intermediate host is to be sought among the various insects and other furms of animal life to which the infested birds have access such as snails, insects, crustaceans or worms.

Symptoms. These vary to some extent in different birds according to age and the degree of infestation. A few worms are not harmful to the bird and can hardly be noticed. Young birds are affected more seriously than old ones. In moderate infestation the bird is always hungry and experiences excessive thirst. Restlessness is marked and doubtless accounts fur the lean condition of the bird. Heavily infested birds show such symptoms as drooping wings, emaciation, ruftled feathers and isolate themselves from the flock.

The condition of the feces is more ur less altered in tapeworm infestation. Heavy infestation causes inflammation of the intestine and diarrhea with mucous droppings. The secretion is at first clear, transparent, semi-liquid and slightly wirisu in colur. Later the mucus becomes brownish yellow in col whe to hemurrhage caused by the worms, and this coloration cons ituies one of the most char- 
acteristic symptoms. When the bird is very heavily infested, gas bubbles are observed in the droppings, and remain risible for some time.

Segments of tapeworms may be found in the feces by careful search, and microseopic examination may be utilized for detecting the presence of tapeworm eggs.

Tapeworm infestation in geese has been reported in Germany as causing very marked nervous symptoms. The birds walk very little and assume an upright position like that of the penguin.

Morbid anatomy. In excessive infestation the carcass is anemic and emaciated. On slitting the intestine, worms will be found in

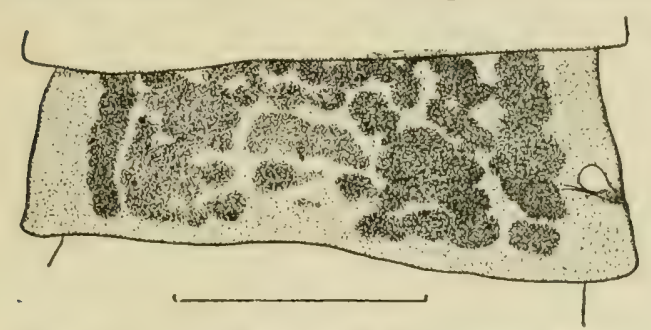

FIC. 25. Gravid segment of Davainea echinobothrida. Enlarged. (Ransom)

nea echinobothrida which results in the formation of nodules in the intestine. This condition has been described by Moore under the name of nodular tæniasis. The nodules are most numerous in the lower third of the small intestine. Exceptionally they occur in the duodenum and colon. In severe infestation the presence of nodules causes numerous protuberances from the serosa, varying in size from those barely perceptible, to elevations $4 \mathrm{~mm}$. high. The color varies from pale or dark yellowish in the larger ones, to the normal gray of the serosa greater or less number throughout its whole extent between the gizzard and the ceca. They are attached to the mucosa by their heads and may cause more or less inflammation. The most serious pathological changes are caused by infestation with Davai

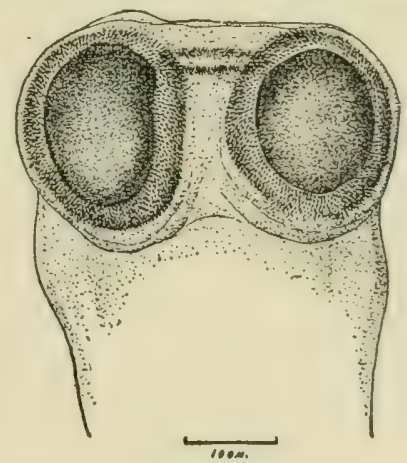

FIG. 26. Head of Davainea echinobothrida. Enlarged. (Ransom) shown by the smallest ones. Similar elevations are observed on the mucosa of the intestine. Small tapeworms oceur attached to the mucosa over the nodules. Over some of the nodules there are areas in which the mucosa has sloughed, leaving ulcers.

The contents of the larger nodules eonsist of greenish yellow, necrotic material which on section has a glistening, homogenenus alp- 
pearance. Surrounding this, there is a thin layer of infiltrated tissue. The smaller nodules contain a substance more resembling pus, and in these the microscopic study of sections most readily reveals the presence of tapeworm heads. The penetration of the intestinal wall by the heads induces infiltration and eventually results in the formation of nodules.

Diagnosis. The chief points to be relied upon in diagnosis are emaciation, excessive appetite and thirst together with the characteristic yellowish-brown color of the droppings. The detection of tapeworm segments in the feces is best done by immersing the suspected white mass in water. If it consists of urates alone, it will be dissipated in fine granules, while the worm segments will be recognized as firm masses. Some of the general symptoms of unthriftiness are also indicative of nematode infestation, but in this case the birds often pass blood. Ultimate decision will rest upon the information

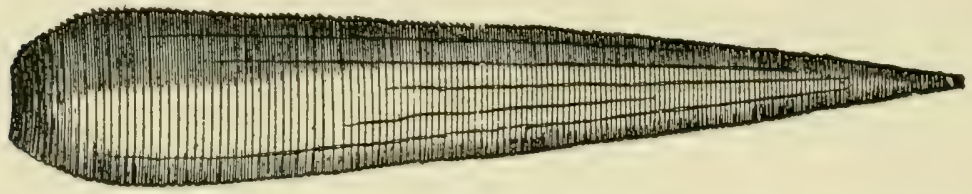

Fig. 27. Hymenolepis lanceolata. Natural size, in a medium state of extension. (Railliet)

yielded by an autopsy. The intestine should be opened while immersed in warm water, to facilitate recognition of the worms. They will be found attached to the mucosa, and vary in size from microscopic objects to worms four or five inches long.

Treatment. Turpentine is held in high repute as a tæniafuge and has the additional advantage of expelling round worms also.

The fowls are fasted for twenty-four hours during which time they should receive a dose of Epsom salts. One teaspoonful of salts is allowed for each bird, the whole being dissolved in warm water and mixed with mash. Greater accuracy of dosage will be assured if the dose is administered directly to each bird. The most convenient system of carrying out the vermifuge treatment is to withhold food in the morning and give the salts in the evening. The following morning the turpentine should be given in a dose of from one to two teaspoonfuls per fowl. Owing to the offensive qualities of turpentine it is not possible to give it in food, but it must be administered to each bird individually. The most expeditious method is to inject the dose directly into the crop with a hypodermic syringe. A slower 
method is to introduce the dose into the crop by means of an oiled muber tube inserted through the oral cavity and esophagus. The turpentine, diluted with an equal amount of olive oil may be administered by the mouth. If the mixture is given slowly with a spoon or medicine dropper the bird will swallow it naturally and will cause very little trouble. Three or four hours after giving the turpentine, the birds should receive another laxative dose of salts administered in a mash as before.

Owing to the habit of burying their heads in the intestinal wall, tapeworms are very resistant to treatment. Consequently it is advisable to repeat the vermifuge treatment in about three weeks.

Gutberlet reports favorably upon the use of lye for expelling tapeworms. A tablespoonful of concentrated lye was added to one gallon of a mixture of wheat and oats which was cooked slowly for two hours. Fifteen birds, after fasting for about fifteen hours, were allowed to eat the mixture. A second dose is given twelve hours after the first one.

A number of other substances are employed to expel tapeworms, but some fail on account of having lost their active properties. Areca nut in doses of 30 to 45 grains may be

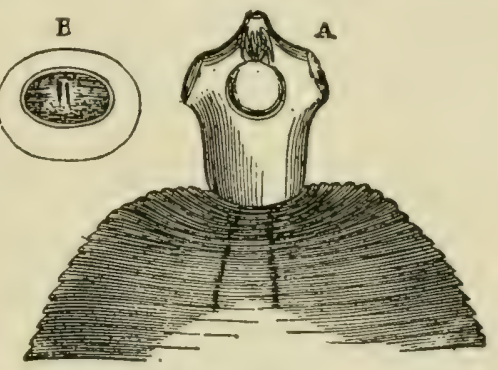

Fig. 28. Hymenolepis lanceolata. A, cephalic extremity, enlarged 100 times; $\mathrm{B}$, egg enlarged 300 times. (Railliet) administered to fowls in the form of a pill, but turkeys do not tolerate this remedy well.

Powdered pomegranate root bark may be fed to fowls in a dose of one teaspoonful to each 50 birds. It may be followed by a purgative dose of 2 or 3 teaspoonfuls of castor oil per bird. Male fern in a dose varying from 30 grains to 1 dram of the powder may be given morning and evening before feeding.

Mrégnin recommends treating pheasants for tapeworm with kamala, mixed into a paste with hard boiled eggs and bread.

Prophylaxis. An accurate knowledge of the secondary host for each species of tapeworm, might suggest very effective preventive measures but in the absence of much of this information, such measures must be of a general nature. It is desirable to collect the droppings and treat with lime. Lime or ashes may be seattered over the droppings under the roosts. General sanitary measures are of value, and particularly, the practice of moring birds to new 
ground should be encouraged. The knowledge that the house fly is the intermediate host of a chicken tapeworm suggests the advisability of combating that insect. It breeds commonly in bird or horse manure and any decaying vegetable matter. These breeding places should be eliminated as far as possible besides using fly traps.

\section{ROUND WORMS}

General nature. Round worms or nematodes are common parasites of the intestines and are capable of inflicting considerable damage. They are long; slender and thread-like in form. Mouth, alimentary canal and anus are present. The female worm is generally larger than the male.

Species of round worms. There are two principal species in fowls. Ascaridia (Heterakis) perspicillum occurs in the small intestine. The male is 3 to $8 \mathrm{~cm}$. long, while the female is 6 to $12 \mathrm{~cm}$. in length. The color of the body is yellowish white. They are often present in numbers sufficient to ocelude the lumen of the intestine. The same species is found in the turkey and guinea-fowl. Heterahis papillosa, a much smaller worm with white body, is very common in the ceca. The male is from 7 to $13 \mathrm{~mm}$. long while the female varies from 10 to $15 \mathrm{~mm}$. in length. This species is found also in the turkey, gninea-fowl, peafowl, and pheasant. Five other less important species, Heterakis lineata, H. brasiliensis, H. compar, $H$. compressa and $H$. differens have been reported by Neumann as occurring in fowls. This writer lists other nematodes of the intestine as follows:

Pigeon: Heterakis columba.

Duck: Ascaris crassa, Heterakis dispar, $H$. vesicularis, $H$. lineata, Strongylus tenuis.

Goose: Heterakis dispar, H. vesicularis, Strongylus tenuis, Trichosoma anatis.

Life history of Ascaridia perspicillum. Eggs are deposited by the worms within the intestine and are expelled in the feces. The length of time worm eggs will remain alive in the droppings is not fully determined, but there is evidence that they are viable up to a year. The life cycle of the worm is completed by eggs gaining access to a fowl through contaminated feed or water. Ordinarily, eggs do not hatch until they are taken into the alimentary canal of a fowl. The worms require three to four weeks to develop into sexually mature forms. 
Symptoms of Ascaridia infestation. Affected birds appear unthrifty and emaciated. Either diarrhea or constipation may occur. The affection is most severe in young birds.

Treatment. Beach conducted experiments with a number of remedies to determine their value in causing the expulsion of the worms and came to the conclusion that tobaceo stems are most efficient. For one hundred fowls he takes one pound of tinely chopped tobacco stems and steeps them for two hours in enough water to keep the mass covered. The liquid and the stems are mixed with half the amount of ground feed usually allowed the birds for one feeding. On the evening before the day of administering the remedy, the birds should be fed only half a ration and they should be kept without feed until afternoon of the following day. Then the mixture of tobaceo and feed is given and under the circumstances is sometimes readily eaten. Occasionally, fowls refuse the mixture. Two hours later, the birds are given a quarter size ration of ground feed mixed with water containing eleven ounces of Epsom salts for each hundred birds. No difference of dosage to currespond to birds of different ages is attempted, for the matter is adjusted by the amounts of the mixtures that the birds can eat. Reasonable care should be exercised to make certain that birds have equal opportunity to eat. . The treatment is calculated to cost about ten cents per hundred fowls.

The same procedure should be repeated in two weeks which has been found to be sufficient to free the birds entirely from parasites. Difficulty in administering the treatment is sometimes encountered because the birds will refuse to eat the mash containing the tobaceo.

Theobald recommends thymol as an agent against nematodes. The dose for the fowl is 06 gram given in the form of a pill. Two or three hours after giving the thymol, a teaspoonful of olive oil is administered.

Mégnin recommends administering santonin in the food in a dose of 4 to 5 grams for every ten birds.

Klee recummends 5 to 10 drops of anise oil in 1 to 2 teaspoonfuls of olive oil, also 4 to 6 drops of benzol with oil.

Prophylaxis. Thorough disinfection of the yards and quarters is necessary in order to minimize the possibility of reinfection from worm eggs that have been deposited in the feces. INerms and Beach have determined that worm eggs are found only in the top two inches of the soil of infected yards. After sweeping the yard and remoring rubbish the area was treated with a 1:1000 solution of bichloride of mercury. About one gallon of solution to each ten square feet of 
soil was found necessary to secure penetration of the fluid to a depth of two inches. A trial of the effectiveness of the treatment in preventing the reinfection of birds that had been freed of worms yielded evidence of its value. Such birds increased in size rapidly and post mortem examination of the poorest specimens after three weeks yielded no evidence of worms. Other fowls on a control area, not disinfected, became seriously infested in a similar length of time.

Bichloride treatment of roosting houses and other buildings to which birds have access, is indicated. Netting under the roosts is of value in keeping birds from contact with feces.

Round worms in the pigeon. Heterakis columbce occurs in the intestines of pigeons in enormous numbers. As many as 400 to 500 worms have been observed in one individual. The infestation causes loss of appetite, periodic mucous diarrhea and emaciation which is

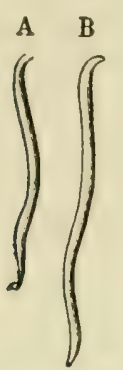

Fig. 29. Heterakis columber. A, male; $\mathbf{B}$, female. Natural size. (Railliet) particularly marked in the pectoral muscles. $\Lambda t$ autopsy the worms are observed packed closely together. The mucosa is distended in patches which are engorged with blood and ulcerated. These areas are covered with thick mucus. Occasionally the parasites perforate the wall of the intestine and are found in various parts of the thoracic and abdominal cavities. Treatment advised by Zürn consists of administering 6 centigrams of calomel in the form of pills with bread or with butter. More serious cases may be treated with areca nut in 1 gram doses, given in the same manner. Two hours after administering the pills, each bird is given a teaspoonful of castor oil. The affected birds may be given a weak decoction of garlic to which one-half per cent of sodium salicylate has been added.

Strongylus quadriradiatus has been observed by Stevenson in the intestine of the pigeon. The presence of a few worms causes no harm, but large numbers cause harm attributed to injury of the wall of the intestine permitting subsequent invasion by bacteria.

\section{TRICHOSOME INFESTATION OF CHICKENS}

Several worms of the genus Trichosoma have been described as harmless inhabitants of the intestines of fowls. However, Freese has observed that Trichosoma retusum is capable of causing great destruction among chickens. The same parasite has been reported in the guinea-fowl. The worms vary from 13 to $19 \mathrm{~mm}$. in length 
and from .06 to $.07 \mathrm{~mm}$. in breadth. Other species reported in the fowl are $T$. collare, T. dubium, $T$. caudinflatum and $T$. gallinum.

Symptoms. The chickens become less lively, allow the wings to droop and show diarrhea. The appetite is at first very good lut later becomes poorer. There is increased thirst. The bird becomes more and more emaciated and finally ean hardly move because of loss of strength. Without treatment the disease usually ends fatally. The duration of the disease varies from three to ten days according to the resistance of the victim.

When old hens are affected, they show similar symptoms. At the beginning of sickness egg laying drops off and soon stops entirely. The disease in hens runs a course of 2 to 4 weeks.

Morbid anatomy. The carcass is greatly emaciated and anemic. All organs with the exception of the intestinal canal, are free from lesions. The mucosa of the small intestine throughout its whole extent is covered with a grayish yellow exudate. The mucosa is thickened and reddened on the surface by short streaks and points. The contents of the ceca and rectum are thin and viscid. Innumerable trichosome worms are present on the mucosa of the small intestine throughout its whole extent, and a few also occur in the ceca.

Diagnosis. The disease caused by the trichosomes can be recognized with certainty only by autopsy. On account of the lair-like fineness of the worms they can only be discovered after the closest scrutiny. The contents of the intestine are teased with a needle, or the needle is stroked over the mucosa. If the worms are present, it is likely that several will adhere to the needle. Another method is to remove a portion of the exudate from the mucosa and mix with water. The hair-like worms may be detected with the naked eye. In any event it is desirable that microscopic examination be made.

Treatment. Freese recommends the administration of oil of anise in early stages of infestation. On each of two successive days each chicken is given a dose of 5 drops of etherial oil of anise in 1 teaspoonful of olive oil. Old hens require twice as much anise oil.

Trichosomes in the pigeon. Trichosoma columber is frequently found in the large intestine of the pigeon. It is capable of causing an intense intestinal catarrh. It may be present in colossal numbers in the intestine causing the mucosa to show tumefaction, infiltration, petechiæ and red streaks. 


\section{FLUKES}

General nature. Trematodes (flukes) are hermaphroditic worms possessing suckers by which they become attached to the mucosa of the intestine of the host. The intestinal tract ends blindly without an anus. In the temperate zone they are generally harmless and uncommon parasites.

Species and hosts. Neumann states that Echinostomum echinatum occurs in the fowl, duck, goose and swan; E. conoideum in the fowl, duck and goose; E. recurvatum in the fowl and duck; Clinostomum commutatum in the fowl, turkey and pigeon; Notocotyle vernucosa in the fowl, duck and goose. Bunodera linearis has been reported in the fowl alone, Cyclocolum mutabile in the turkey alone, while Holostomum gracile and $H$. spharocephalum occur in the duck alone.

\section{THORN HEADED WORMS}

Three species of the genus Echinorhynchus occur in ducks but only one has been reported as causing injury.

Echinorhynchus polymorphus is fusiform in shape and colored orange red. The anterior extremity of the worm is provided with an ovoid proboscis armed with eight rows of hooks. The male worm measures 4 to $6 \mathrm{~mm}$. in length while the female varies from 15 to 25 $\mathrm{mm}$. in length. The worms usually are fixed firmly to the wall of the intestine.

Before opening the intestine the serous surface of the same is seen to be covered with numerous nodules, the centers of which are orange colored. On opening the intestine the mucosa is found studded with worms of the same color attached by their anterior extremities. Their presence sets up a severe inflammation of the mucosa and causes the formation of the nodules on the serous surface. The condition described often causes diarrhea, emaciation and anemia.

The same species occurs in the swan. E. filicollis and E. spoerocephalus are found in the duck.

\section{FLAGELLATES}

Flagellated protozoan organisms constitute a small number of forms parasitic in the intestines of birds. These are nucleated bodies provided with one or more flagalla and sometimes possess an undulating membrane. Neumann lists the following species: Trichomonas eberthi has been observed in the glands of Lieberkuhn of a duck and in a goose. It is little known and without pathogenic 
importance. T' columbe has been reported as causing the formation of caseous foci in the liver of the pigeon with fatal result. Monas (Monocercomonas) anatis has been reported as occurring in the small intestine of the duck. Martin and liobertson have studied the flagellates of the ceea of fowls dividing them into the four species: Chilomastix gallinarum, Trichomonas gallinarum, 'T'. eberthi and Trichomastix gallinarum.

Hadley observes that the intestinal fauna of any young turkey after it has been on the ground for a few days will contain a varicty of protozoan parasites. Among these, the most common are flagellates, certain sporozoa and amebas. At least three types of flagellates are usually present, Trichomonas, Chilomastix and Lamblia. He considers that Trichomonas plays a pathogenic rôle in cecal and hepatic infection in birds comparable to intestinal and hepatic amebiasis as observed in mammals. Hadley considers the flagellates described by him as identical with bodies described by Theobald Smith under the name Amoba meleagridis and the canse of blackhead in turkeys. He regards Trichomonas as a facultative parasite in that under certain conditions it exists as a harmless, ever-present intestinal parasite. According to this view the Trichomonas under certain unknown circumstances invades the tissues and causes disease.

Trichomoniasis of chicks. Teinzirl has described a highly fatal disease of chicks attributed to a new species designated Trichomonas pullorum.

Symptoms. Chicks under a month old only are attacked. 'The disease most commonly appears about the tenth day after hatching. The affected birds droop, they huddle together, the wings drag, the eyes are closed and the skin of the head is pale. Acute cases succumb in a day or two without showing diarrhea. Other cases become chronic, in which stage diarrhea is present. The temperature is subnormal and is usually about $102^{\circ} \mathrm{F}$. The disease is not observed until late spring and summer.

Morbid anatomy. Autopsy reveals no lesions except a general anemic condition. The ceca may be slightly enlarged and the contents thin and slimy. The parasites collect in the erypts of the mucosa frequently in pure culture. When diarrhea exists, the protozoan may be found in the intestine and in the voided mueus.

Etiology. The flagellate Trichomonas pullorum regarded by Weinzirl as the cause of the chick disease occurs in two forms. One, the pear shaped type moves about actively by means of flagella and also exhibits active ameboid movements. This type varies from 3.5 
by 7 microns to 5.5 by 9 microns, and is the only form occurring in the cecal crypts. The globular form occurs in the lumen of the ceca.

Control of disease. Feeding of sour milk is favorably regarded as a means of saving life.

\section{PARASITES OF THE LIVER}

The liver of birds is rarely subject to parasitism, if entero-hepatitis of the turkey be excepted.

A flagellate Cercomonas hepatica has been reported in the liver of squabs, causing yellowish nodules.

Echinococcus polymorphus, the cystic phase of the tapeworm of the dog, occurs sometimes in birds, and exhibits a preference for the liver. Echinococci have been reported in the goura, the peafowl and the turkey. The liver becomes enlarged by the presence of the cysts until it occupies a large part of the abdominal cavity. The hepatic tissue is reduced to thin pieces between the cysts.

Three species of flukes, Opisthorchis simulans, Metorchis xanthosomus and Bitharziella polonica have been found in the hepatic canals or biliary vesicles of the domestic duck. The latter species occurs in the blood of a large number of species of wild ducks.

Heterakis columba, normally a parasite of the intestines of pigeons, has been reported as occurring in nodules in the liver.

Blastomycosis. Martin and Daille have described lesions in the liver of the goose, due to Blastomyces (Cryptococcus) anseris. The parasites are contained in small sacs about the size of a pea, attached to Glisson's capsule. These sacs are yellowish white in color, fluctuate on pressure and are connected with one another by narrow channels. The contents of the sacs consist of a yellowish white gelatinous material enclosing the blastomrces. These latter consist of round or oral nucleated refractile bodies surrounded by a distinet membrane.

\section{PARASITES OF THE BURSA OF FABRICUS}

According to Neumann, the bursa of Fabricus harbors species of flukes of the genus Prosthogonimus. They are encountered there but rarely, for that pouch disappears in the adult. The parasites consequently are obliged to leave that diverticulum and enter some of the other canals connecting with the cloaca. Sometimes they enter the rectum, or they may penetrate the oviduct and become enclosed in an egg. Probably all the forms found in the oviduct and the egg also occur in the bursa of Fabricus. 
Prosthogonimus ovatus occurs in the bursa of Fabricus of a large number of birds. It has been found in the oviduct of the hen and many observers have found it in hen's eggos. Pr. pellucidus and $P r$. japonicus have been found in eggrs. P'r. cuneutus has been reported as occurring in the oviduct of a pea hen.

\section{COCCIDIA}

Coccidia occurring in the intestines are discussed under the heading coccidiosis, page 127.

\section{PARASITES OF THE NASAL CAVITY}

A species of mite of the genus Rhinonyssus infests the nasal cavity of fowls, causing a sort of coryza. The mites also occur in squabs which sometimes succumb to the parasite.

Sternostomum rhinolethrum occurs in the nasal fosse of the duck.

A leech Hemiclepsis tessellata occurs in the nasal and oral cavities of geese and ducks, attached to the mucosa. The parasites also attach themselves in the eyes, esophagus, larymx and trachea. The affected birds are tormented by the presence of the parasites and rapidly become emaciated. Mégnin recommends applying a two per cent salt solution to cause the detachment and death of the parasites.

A fluke Cyclocolum arcuatum is reported as a harmless parasite of the infraorbital sinus of the domestic goose.

\section{GAPE WORMS}

Gapes is caused by the roundworm designated Syngamus trachealis which attaches to the mucous membrane of the trachea and causes severe inflammatory changes. These lesions and the additional factor of mechanical blocking of the air passages may canse death. The air sacs are sometimes infested.

Species affected. The fowl, pheasant, turkey, peafowl, pigeon, certain cage birds and a considerable number of wild birds have been reported as hosts of Syngamus trachealis. The adult turkey appears to be very much more frequently infested than the adult fowl. Ransom found about 23 per cent of a number of turkeys infested when no parasites were found in 635 chickens examined. A related specie's S. bronchialis occurs in the trachea, air saes and bronchi of young geese. 
General character. The female worms are about four-fifths of an inch in length and reddish in color. In most cases, the male, about a quarter of an inch long, is in copulation, attached to the female near the anterior extremity. Thus the appearance of a forked worm is induced.

Symptoms. The presence of the worms is indicated by the peculiar wheezing cough, gasping and the expulsion of frothy saliva from the beak. The birds appear dull with ruffled feathers, lose appetite, breathe with diftieulty and displar cachexia. Spontaneous recovery is rare, and in the absence of treatment the parasites cause heavy mortality. Only young birds experience harm from their presence.

Morbid anatomy. The presence of the parasites in the air passages enveloped in foamy mucus is the most important feature observed at autopsy. The point at which the parasites adhere often consists of an abscess filled with yellow colored pus of caseous consistency. Such an abscess may cause asphyxia.

Life history. The sexually ripe females are expelled by coughing, whereupon the eggs are disseminated by the disintegration of the female. Thus water and soil become contaminated with the larva hatching from the eges. It is not believed that an intermediate host is necessary in the life cycle of the parasite, even though it has been shown that the feeding of earthworms will induce the disease amme chicks otherwise protected against infection. L'ndoubtedly earthworms may contain larve in the intestinal tract, due to earth ingested by them, without however, playing the rôle of a host. It has been demonstrated repeatedly that chicks may be infected by drinking boiled water artificially infected with ova and young gape worms. The young worms may live for some time in water, and the persistence of the infection is favored by a wet soil. Infection undoubtedly occurs through ingestion. The embryos probably make their way frem the gullet or crop, to the trachea by perforating the walls of the organs in question.

Treatment. The practice of dislodging the worms by the application 'f rarinus remedies applied by inserting the tip of a feather into the trachea and twisting, is widespread. The small size of the trachea in young chicks and the fact that some worms are located at its lower extremity out of reach, makes the operation painful and uncertain in its results. Among the remedies applied are oil of cloves, ril $\circ$ f turpentine thinned with double its volume of olive oil "nd kep"sme nil. The feather also acts mechanically in dislodging 
the parasites, wherenpon they are expelled hr conghing. A coiled horse hair is frequently employed for the same purpose, but like the feather its use is dangerous to the chick. A few worms may be caught with tweezers if the larynx is raised high enough.

It has been asserted that pounded garlie in the proportion of one bull, per day added to the food of ten birds would result in the expulsion of the worms. Asafoetida has been administered in the food with the idea that the agent, eliminated through the air passages, would kill the parasites.

Fumigation with various agents has been emplored. Exposure to sulphur fumes has been used to induce coughing and expulsion of the worms but is dangerous. Affected birds may be exposed to tobacen smoke until they beenme almost insensible, after which they are allowed to recover in the open air. Fumes of heated carbolic acid hare been employed.

Klee has great confidence in intra-tracheal injection which requires no more time than other more dangerous kinds of indiridual treatment and is regarded as more effective. The affected bird is injected in the trachea with 1 c.c. of a 5 per cent aqueous solution of sodium salicylate, administered with a hypodermic srringe. This fluid simultaneously loosens all the parasites occurring there and a

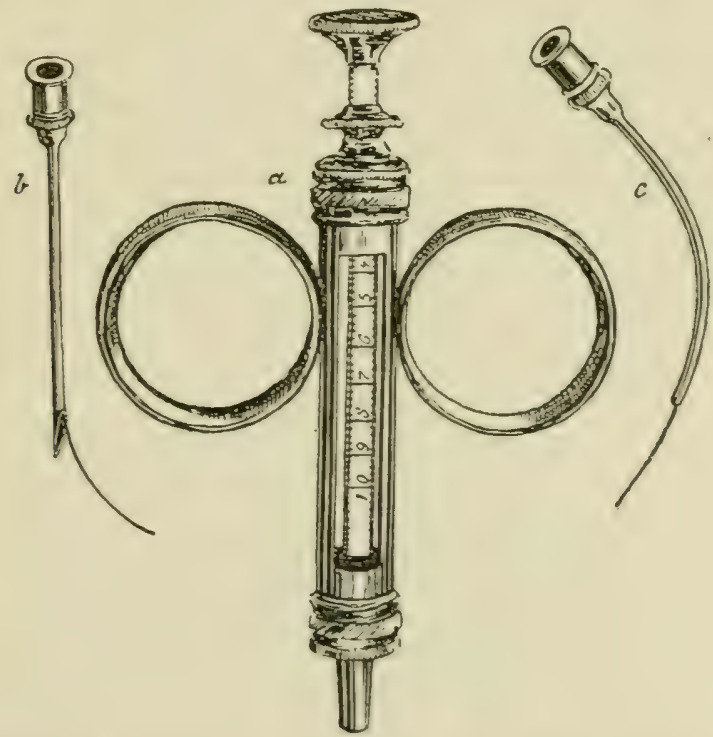

FIs. 30. Apparatus for making injections in trachea. a, Syringe; b, Straight canula; c, Curved canula. (Filee) 
violent cough will expel them. The operation in question can be performed by one person, for a syringe with two rings on the barrel can be guided easily with one hand. The sick bird is held between the knees with the breast in front. The neck is bent up and back with the left hand and the trachea lies between the thumb and index finger. Next, the syringe lying conveniently, is grasped with the right hand. The needle is placed near the trachea, after which with necessary care it is inserted into the trachea slanting downwards and the injection made quickly. The introduction of a dull canula into the glottis through the beak is even more suitable than the foregoing method. After the injection the bird suffers a light attack of choking but soon recovers.

A very safe but a somewhat less effective remedy is to cause the affected birds to breathe air containing finely powdered chalk and camphor. Theobald recommends a mixture of two parts by weight of powdered chalk and one part of powdered camphor. This is dusted into a closed box in which the chickens are confined.

Prevention. Contaminated water and soil are to be regarded as the chief sources of infection. Infectious larve have been kept alive for a year in moist soil. Chicks raised on board floors and thus isolated from the soil with separation from older birds until a month or six weeks old, will escape infection provided the water and food supply are not infected. Rotation in the use of land to which chicks are allowed access is a valuable practice in avoiding the use of contaminated soil from year to year. Above all, chickens should not be kept on soil that has been occupied by turkeys within a year or so. Disinfection of drinking vessels and of soil, so far as possible, deserves consideration. Salicylate of soda, 3 drams to the quart of drinking water is said to kill the embryo worms. Contaminated soil may be disinfected by drenching with a one per cent solution of sulphuric acid. Wet areas should so far as possible be dried by draining.

The isolation of the sick and thorough disposal of the dead are obvicus precautions.

\section{THE AIR SAC MITE}

Cytoteichus (Cytodites) nudus has a wide distribution in the United States and Europe affecting a large number of gallinaceous fowls and also pigeons. The mites are barely large enough to be detected by the unaided eye. They have been observed in the trachea, 
lungs, air sacs, hollow bones and the peritoneum. They have been reported as occurring exceptionally in the heart, liver and kidneys.

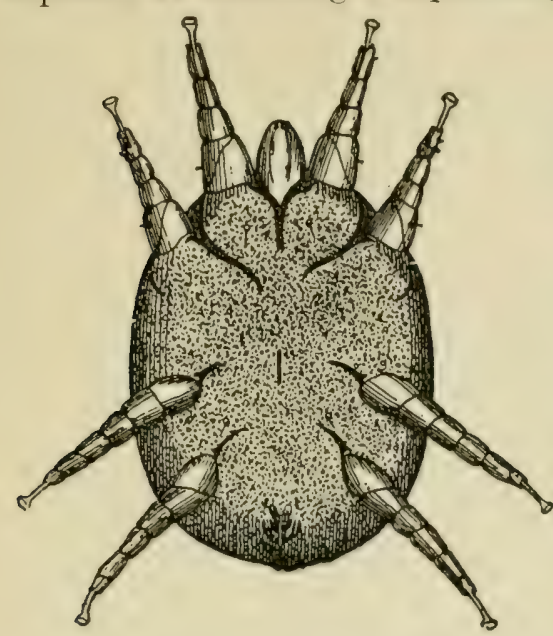

Fig. 31. Cytodites nudus. Male, ventral aspect. Enlarged 100 times. (Railliet) pear to be of pathological importance. Gerlach regarded them as the cause of enteritis as did also Zundel. Williams attributed high importance to them as a parasite

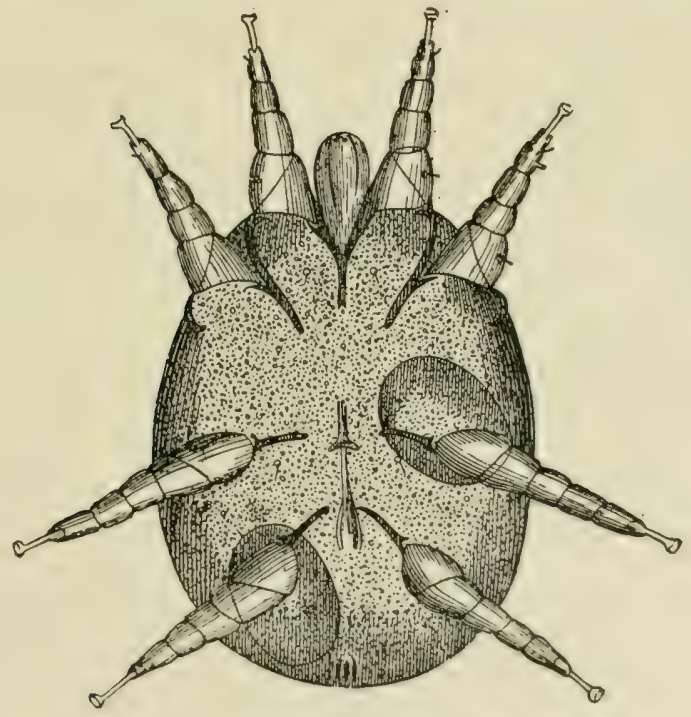

FIG. 32. Cytodites nudus. Ovigerous female, ventral aspect. Enlarged 100 Their presence within tissues is not readily explainable since their structure is not adarted to the penetration of tissue. They are most commonly noted in the air sacs. Hens apparently in perfect health may be heavily infested, so that the economic importance of the parasite seems to be not very great. The method of transmission has not been thoroughly explained. Their exit from the body could oceur through the trachea by expulsion during coughing.

A few writers have observed cases in which the parasites ap- 
of fowls in Montana, a view that Wilcox was not able to confirm.

Among the later observers Walker has reported upon two autopsies of fowls in Grahamstown, Cape Province, in which lesions occurring in the lungs, were in his opinion, the cause of death. The owner reported that the comb turned purple, appetite was lost and the birds. seemed fretful and weak in the legs. Birds were noticed to be sick for a week, when sudden collapse oceurred. Observations by Walker follow.

Morbid anatomy. Cytodites are frequently found in the thorax and peritoneal cavity, fairly frequently on the mesentery and in the trachea. The lungs are mottled in appearance by some purple patches .2 to $.3 \mathrm{~cm}$. in diameter. On section, hyperemia is observed and serous fluid exudes from the eut surfaces. Small miliary tubercles of a gelatinous consistency are scattered through the affected portion of the lung, and parasites are visible in the midst of each of these. The larger bronchi and some of the bronchioles contain an exudate in which the parasites are imbedded. Other organs and blood appear normal. The conditions observed warrant the pathological anatomical diagnosis of hyperemia and edema of the lungs, and bronchial pneumonia.

Microscopic examination of sections of stained tissue, under 300 magnification shows that the lung tissue surrounding the invaded air passages is infiltrated with leucocytes and red blood corpuseles.

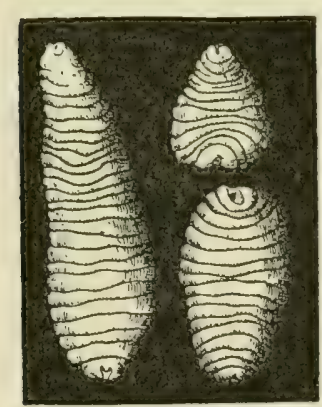

FIG. 33. Dithyridium variabile, from a fowl. Enlarged 10 to 12 times. (Neumann) The blood vessels are congested. Some of the parasites have wandered from the air conduits into the surrounding parenchyma breaking down the regular network conformation of the latter. In some of the invaded air passages, the parasites have lodged themselves in the inner lining and have broken it down. In places cytodites are to be seen in the interlobular fibrous tissue. The larger bronchi contain an exudate.

\section{CESTODE CYSTS IN THE LUNGS}

Neumann has described a case in which the encysted parasite known as Dithyridium variabile occurred in great numbers in the lung of a fowl. These represent the cystic stage of a tapeworm, which has not been identified with the adult form. The cysts were observed in the inferior surface of the lungs in the an- 
terior and posterior diaphragmatic reservoirs. They wore globular or oroid bodies 3 to $4 \mathrm{~mm}$. in diameter, somewhat transparent and filled with a colorless liquid containing a parasite. Some were free, and others adhered slightly to adjoining tissues. The dithyridium has a milk-white body, varying in length from 1.5 to $2.5 \mathrm{~mm}$. It is sometimes as broad as long and sometimes much longer than broad. An invaginated cestode scolex is present.

\section{MANSON'S EYE WORAI}

At least 38 species of nematode worms have been reported as parasitic in the eyes of birds throughout the world. However, only one, Oxyspirura mansoni, is known to affect common domesticated birds. This has been found in fowls and peafowls. Ransom has reported upon its presence in the United States, and upon the prevalence of nematodes in wild birds.

General description. The parasite known as Oxyspirura mansoni or Filaria mansoni or Spiroptera emmerzii is a small white, thread-like

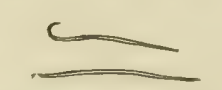

Fig. 34. Manson's eye worm (Oxyspirura mansoni). II a le above and female below. Natural size. (Ransom) worm slightly over half an inch in length and about as thick as a fine scwing needle. It is thickest in the middle and tapers toward the ends. The number present in the eyes may vary from a few located beneath the nictitating membrane to as many as 200 .

Symptoms. In some cases the worms are present in the eyes without causing apparent inconvenience. In more severe infestation, symptoms of irritation become evident. The affected bird appears uneasy and scratches the eyes. The latter show acute inflammation with abundant lachrymation. Subsequently white cheesy exudate collects within the conjunctiva and upon the edges of the lids, causing them to adhere together. The inflammation extends to the tissues surrounding the eye and to the infra ocular sinuses. Erentually the whole eyeball becomes affected, with consequent destruction of the organ. Catarrhal changes also involve the nasal cavities. In severe cases the subject dies.

Treatment. The first requisite to successful treatment consists in removing the worms. This may be done by the use of forceps or by irrigating the eyes with some solution such as bicarbonate of soda or a two per cent solution of creolin. When the worms are partially dislodged by the fluid, they may be entirely remored with a soft cloth. The inflammation may be alleviated by instilling into 
the eyes a few drops of saturated solution of boric acid. Ransom recommends anointing the eyes with a mixture of lard 9 parts and iodoform, 1 part or with carbolized vaseline.

Life history of the worm. Very little is known of the life history of the parasite. It seems probable that the embryos pass a portion of their existence either free or in a secondary host. The reports of the presence of the worm in chickens are, so far as known, limited to sea coast regions. This suggests the possibility that some conditions peculiar to the sea coast are necessary to the development of the parasite.

\section{LEECHES IN THE EYE}

One incomplete report exists of the occurrence of leeches in the eyes of geese in Ireland. Intense irritation of the eye occurred, with symptoms of severe pain. The birds had access to a pond teeming with leeches.

MITES PARASITIC IN CONNECTIVE TISSUE

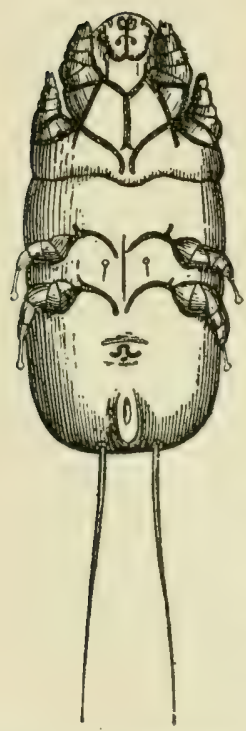

FIG. 35. Laminosioptes cysticola. Male, ventral aspect. Enlarged 200 times. (Railliet)
Laminosioptes cysticola, according to Neumann, is a mite occurring in gallinaceous birds such as fowls, pheasants and turkeys. The parasites live on the surface and in the thickness of the skin causing the formation of scurf. They are encountered rarely except in the subcutaneous tissue especially in the places where it is very loose, such as the flanks, the vent, on the thighs, the breast and the neck. They are able to penetrate the deep connective tissue. Microscopic examination of fragments of subcutaneous connective tissue permits all phases of their development to be observed. When they die, the carcass acts as a foreign body and causes the formation of a miliary nodule about it. This promptly undergoes calcareous infiltration. On treating these nodules with

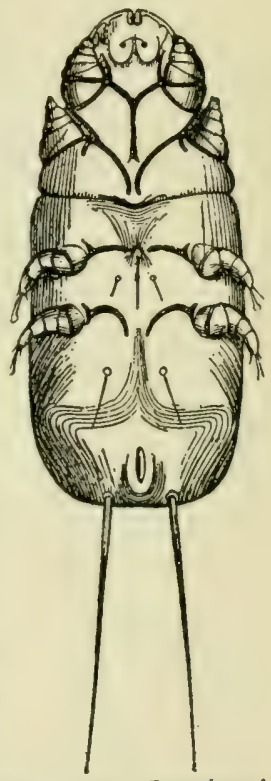

FIG. 36. Laminosioptes cysticola. Ovigerous female, ventral aspect. Enlarged 200 times. (Railliet) 
acidulated water, the remuants of the parasite may be recorered. The nodules are yellowish in color, oval in shape and may become as large as $1 \mathrm{~mm}$. in diameter. They may be very numerous in an individual and are very common in old cachectic birds. These parasites are not injurious to health except perhaps when they are present in excessive numbers.

Two other connective tissue parasites occur in the pigeon. A feather mite, Falculifer rostratus, spends a portion of its life cycle in the subcutaneous comnective tissue. Filaria clava is located in the subcutaneous connective tissue of the pigeon. Neither have any effect upon the health of the host.

\section{TRYPANOSOMIASIS OF BIRDS}

The blood of a very large number of wild birds is infested with trypanosomes which apparently do not exert much pathogenic effect. Gray lists nine species of these parasites occurring in birls. Among domesticated birds, they have been observed in the fowl and pigeon.

\section{FILARIA IN - THE BLOOD}

Filaria anatis has been observed in the heart of a duck. Embryos of filaria have been observed in the blood of a pigeon.

\section{PARASITE OF AVIAN MALARIA}

Symptoms. The parasite of Danielewsky, Hcmoproteus danielewshyi has also been designated Homomoba danielewshyi, Halteridium danielewskyi and Plasmodium danielewskyi.

General nature. The parasite of avian malaria, one of the hematozoa of birds, has many points of resemblance with the parasite of malaria of man.

Distribution. The parasite occurs in a large number of wild birds. Cardamatis examined the blood of 38 species of birds in Greece, including 936 specimens. Of 724 local birds, 21.79 per cent were affected. Of 212 migratory birds, 32.07 per cent were infected. The domestic pigeon is the only domesticated bird reported as infested by the parasite. Aragão refers to the parasite of the pigeon as a separate species, $H$. columboe.

Symptoms in wild birds. Cardamatis observes that birds intensively infected appear less lively, the plumage is dull and emaciation causes them to appear smaller. 
Lesions in wild birds. The organs according to Cardamatis, show characteristic alterations resembling those caused by malaria in man. The blood of sick birds is sometimes watery, less abundant than normal, pale, and coagulates slowly. The spleen is colored like
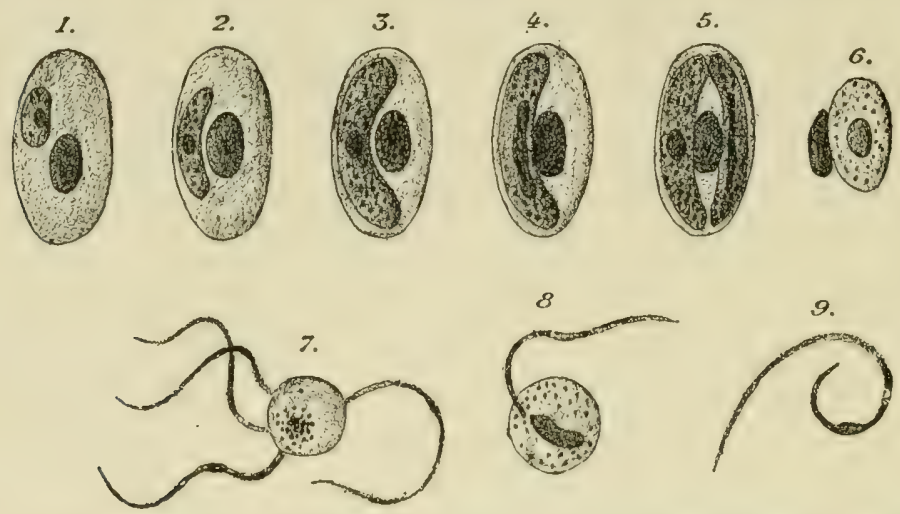

FIG. 37. Hamoproteus danieleusky in blood of the pigeon under different aspects. 1, 2, red blood corpuscles containing small parasites; 3 , red blood corpuscle containing a large female parasite; 4, red blood corpuscle containing a large male parasite; 5, corpuscle with a female and a male parasite; 6 , parasite after rupture of the corpuscle which had contained it (The nucleus of the cell is still seen coupled with the parasite); 7, male parasite with four flagella; 8, female parasite into which a flagellum is penetrating (fecundation); 9, a free flagellum. Enlarged 1500 diameters. (Neumann)

chocolate or darker and is always swollen to twice the normal size. This writer found turtle doves and lanners most susceptible. Iturbe and Gonzales note that while the hematozoa frequently have no effect upon the host, the mortality may reach 90 per cent in Myiozetes texensis, Chlorophonia prettii and Sycalis flaveola.

Transmission. Aragio observes that the natural transmittors of H. columbe in Brazil are Lynchia livicolor and Microlynchia persilla; in Algeria, L. maura; in the Transvaal, L. capensis. Direct inoculation from pigeon to pigeon fails.

\section{LEUCOCYTOZOA}

Lencocytozon are blood parasites which so modify the appearance of the host cell that the identity of the latter is in controversy. Fantham inclines to the view that small mononuclear leucocytes are the cells invaded. Leucocytozoa have been observed in a large number of wild and domesticated birds. Among the latter, fowls, pigeons, ducks, ostriches and turkeys are known to be infested. In general they are of slight importance as disease producers. 


\section{LEUCOCYTOZOÖN INFECTION IN DUCKS}

Wickware observed an outbreak of disease among young ducks under circumstances that led him to suspect that leucocytozoa were responsible. Bacteria could not be excluded as a cause, for it was not possible to seed culture media.

Symptoms. The affection runs a rapid and fatal course, with few symptoms to indicate its onset. Impaired appetite is first noticed in that the birds refuse to respond to the call for feeding. In some cases ducks succumb during the first paroxysm, while others survive a series of exacerbations. Affected ducks lie in a semicomatose condition with the neck bent backwards and the head resting upon the dorsal portion of the spinal column. When roused from this stupor the bird experiences a period of intense excitement during which remarkable movements are made. The head is held in various positions, or describes circles in the air, or at other times swings to and fro. In some cases the neck is completely turned on itself with the head resting on the ground in an upright position. 'The power of equilibrium is lost and the bird turns over backwards until exhausted. In recovered birds, there is lameness and consequent diffienlty of locomotion. Purulent ophthalmia with adherent lids is a quite constant symptom. The mortality is about 65 . to 70 per cent.

Etiology. The leucocytozoön present in the blood of ducks in two outbreaks of disease is designated by Wickware as Leucocytozoön anatis. The shape of the parasites is fairly uniform although there appears to be considerable pleomorphism in the gamete forms. The predominant type is a spindle shaped organism 35 microns to 60 microns in length by 10 microns in width, showing an oval elongated or irregularly shaped nucleus, with dark chromatic band extending along one border. With Giemsa, the nucleus stains a dark blue and has a granular appearance. In the center there may be observed a small chromatin staining body varying slightly in shape. The cytoplasm appears almost transparent or at most stains a faint pink. At each end it terminates in an acute angle. Considerable variation occurs in the staining characteristics of the mature forms.

Morbid anatomy. Other than acute hemorrhagic inflammation of the large intestine behind the eeca, no abnomality of the viseeral organs was observed. The blood in all cases of infection showed an increase of eosinophiles, besides the lencocytozoa. 


\section{REFERENCES}

1. Aragão. Pesquizas sobre o "Hæmoproteus columbæ." Brazil-Medico. 4 et 11 Nov., 1916. Abs. in Bull. de l' Inst. Pasteur, T. 15, p. 300.

2. Cardamatis. Le paludisme des oiseaux on Grèce. Étude biologique et histologique du parasite de Danielewsky. Centralbl. f. Bakteriol. (Etc.), 1 Abt. Orig., Bd. 59, 1909, S. 351.

3. Ciurea. Nematoden aus dem Pharynx und Ösophagus des Haushuhn. Ztschr. f. Infehtionshr. d. Haustiere., Bd. 15, 1914, S. 49.

4. Freese. Ueber die durch den Strongylus nodularis hervorgerufene Magenwurmseuche bei jungen Gänsen und die durch Trichosomen ('Trichosoma retusem Railliet) verursachte Darmwurmseuche. Deutsche tierärztl. Wchnschr., Bd. 16, 1908, S. 713.

5. Gage and Opperman. A tapeworm disease of fowls. Md. Agr. Exp. Sta. Bull. 139, 1909.

6. Gutberlet. Studies on the transmission and prevention of cestode infection in chickens. J. Am. Vet. M. Ass., Vol. 2, 1916, p. 218.

7. Hadley. The rôle of the flagellated protozoa in infective processes of the intestines and liver. Rhode Island Agr. Exp. Sta. Bull. 166.

8. Hadley. The avenue and development of tissue-infection in intestinal trichomoniasis. Rhode Island Agr. Exp. Sta. Bull. 168.

9. Hadley. The case of trichomonas. Amer. Nat., Vol. 51, 1917, p. 209.

10. Hadley. The part played by the goblet cells in protozoan infections of the intestinal tract. $J$. Med. Research, Vol. 36, 1917, p. 79.

11. Herms and Beach. Round worms in poultry-life history and control. Univ. Cal. Agr. Exp. Sta. Circ. 150.

12. Iturbe and Gonzales. El paludismo de las aves en Venezuela. Lab. du Dr. Iturbe, Caracas, 1916.

13. Martin et Daille. Sur une blastomycosis Hepatique de l' Oie. Rev. Vét., T. 7, 1912, р. 129.

14. Martin and Robertson. Further observations on the crecal parasites of fowls, with some reference to the rectal fauna of other vertebrates. Quart. J. of Micr. Sc., Vol. 57, 1912, p. 53.

15. Moore. A nodular tæniasis in fowls. U. S. Dep. Agr. Bureau Animal Indust. Circ. 8.

16. Neumann. Parasites et maladies parasitaires des oiseaux domestiques. Paris: Asselin et Houzeau. 1909.

17. Ransom. Manson's eye worm in chickens. U.S. Dep. Agr. Bureau Animal Indust. Bull. 60.

18. Ransom. A new nematode (Gongylenoma ingluvicola) parasitic in the crop of chickens. U.S. Dep. Agr. Bureau Animal Indust. Circ. 64.

19. Ransom. The tapeworms of American chickens and turkeys. $U . S$. Dep. Agr. Bureau Animal Indust. Circ. 85.

20. Robertson. Notes on ostrich parasites. Agr. Jour. Cape of Good Hope, Vol. 33, 1908, p. 583.

21. Stiles. Report upon the present knowledge of the tapeworms of poultry. U. S. Dep. Agr. Bureau Animal Indust. Bull. 12.

22. Theobald. The gape worm and the white intestinal worms of poultry. Jour. Bd. Agr., Vol. 6, 1899, p. 157. 
23. Theobald. Parasitic diseases of poultry. London: Gurney \& Jackson, 1896.

24. Walker. A short note on the occurrence of a leucocytozoön infection. Host the ostrich. Union of So. Africa. Dep. Agr. Ann. Rpts. Dir. Vet. Res., 2, 1912, p. 384 .

25. Weinzirl. Trichomoniasis of chicks: a new and highly fatal disease. J. Bact., Vol. 2, 1917, p. 441.

26. Wickware. Intestinal parasites of poultry, their prevention and treatment. Dominion of Canada, Dep. of Agr. Health of Animals Branch. Bull. 25, 1917.

27. Wickware. Is Leucocytozoön anatis the cause of a new disease in ducks? Dep. Agr. Canada. Rept. Vet. Dir. Gen., 1915, p. 95.

28. Wilcox. The internal chicken mite. Montana Agr. Exp. Sta. Bull. 22, p. 30. (Bibliography.)

29. Williams. The air sac mite of the fowl. Am. Vet. Rev., Vol. 22, 1898, p. 8. 


\section{CHAPTER XVII}

\section{EXTERNAL PARASITES}

IICE

General nature. Bird lice are wingless parasitic insects with mouth parts adapted to biting, not sucking. Lice in general permanently infest the host bird except when moving to other individuals. Opportunity exists for this migration on the roost, the nest, during copulation or while a hen is hovering chickens. Occasionally lice which are normally parasitic for the hen, guinea-fowl or turkey will be found on another species in close contact.

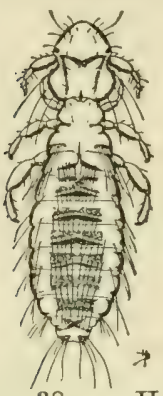

FIG. 38. Head louse. Male, top view. Greatly enlarged. (Bishopp and Wood)

Lice feed on epidermal products such as feathers, scales and the skin itself. Blood dried on the skin may be eaten but lice are not blood suckers.

Injury caused by lice. Their presence causes pruritis set up by their claws and mouth parts. Excessive infestation undoubtedly is a serious hindrance to normal growth and development. Brood hens are annoyed in some cases to such an extent that there is interference with incubation. In the Southern States, there is a heavy mortality among chicks hatched by hens after April 1, which may be attributed to the heary infestation by lice. Bishopp and Wood regard symptoms of lice infestation as consisting of droopiness, lowered wings and ruffed feathers. In grown fowls heavy infestation may be tolerated without causing ill effect. In other cases there is reduction in egg yield and loss of weight.

Species of lice. More than 40 species of lice occur on domesticated birds. Some species are restricted to one host, while others infest several different host species. Bishopp and Wood state that seven species are very commonly found on chickens in the United States, four or five on pigeons, two or three each on geese and ducks, three on turkeys and several each on guinea fowl and peafowl. These are: On chickens, the head louse (Lipeurus heterographus), the body louse (Menopon biseriatum), the shaft louse (Menopon pallidum), the wing louse (Lipeurus variabilis), the fluff louse 208 
(Goniocotes hologaster), the large hen louse (Goniocotes abdominalis), the brown chicken louse (Goniodes dissimilis); on turkeys, the large turkey louse (Goniodes stylifer) and the slender turkey louse (Lipeurus meleagridis). The common body louse and the shaft louse of chickens sometimes are found on the turkey. On ducks Docophorus icterodes and Lipeurus temporatis are common; on geese a variety of $D$. icterodes; on pigeons the slender pigeon louse (Lipeurus baculus) and the broad pigeon louse (Goniocotes compar) cause most of the harm from lice.

Neumann lists 6 species as occurring on the pheasant as follows: Lipeums variabilis, Goniodes colchicus, G. truncatus, Goniocotes chrysocephalus, Menopon productum, and M. biseriatum; on the peafowl, Goniodes falcicornis, G. parviceps, Goniocotes rectangulatus and Menopon phceostomum; on the guinea-fowl, Lipeurus numida, Goniodes numidianus, Goniocotes rectangulatus, Menopon numidoe and Lipeurus variabile of the fowl. In addition to the two species listed by Bishopp and Wood as important lice on the pigeon in the United States, Neumann mentions Goniodes minor, G. damicomis, Menopon giganteum, $M$. longicephalum, Liotheum longicaudum and $M$. biseriatum of fowls as occurring on pigeons; on geese, Lipeurus crassicornis, $L$.

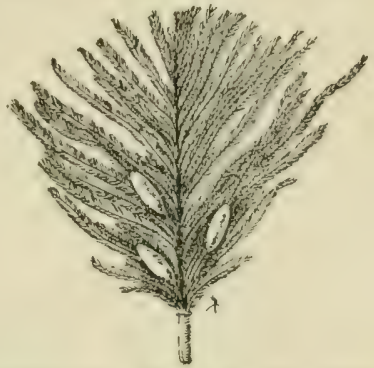

FIG. 39. Eggs of the head louse (Lipeurus heterographus) on feather. Greatly enlarged. (Bishopp and Wood) anseris, Trinoton anseris and T. lituratum; on ducks, $L$. anatis, $T$. luridum and Menopon obscurum; on swans, Omithonomus cygni, Philopterus cygni and Trinoton anseris of the goose.

The terms head louse, body louse, wing louse, etc., as applied to the lice of fowls indicate where the various species are found in greatest numbers and where their structure best adapts them to lire. Thus the head louse exhibits a preference for the head but is occasionally found on the feathers of the wings. Some lice are adapted to live on the skin only, while others cccur on the feathers as much as an inch from the skin.

Life history. In general, lice spend their whole life on one host. All the finer details of the life history of lice have not been worked out. The eggs, attached to the feathers are commonly known as nits. In the case of the head louse they hatch in four or five days. The young, after molting the skin several times, reach the adult stage in 17 to 20 days. 


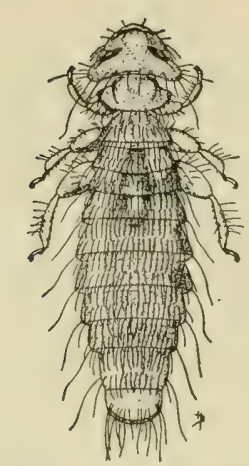

Fig. 40. Body louse (Menopon bis eriatum): Male, top view. Greatly enlarged. (Bishopp and Wood)

Combating lice. Owing to the habit of lice of remaining upon the host, the most direct method of combating them consists of the application of an insecticide to the bird.

Bishopp and Wood favor starting a campaign against lice in the late summer or early fall. The weather conditions are favorable and the amount of stock to be treated is the lowest of any time in the year. There is no reason to prevent a flock from being entirely freed from lice and kept free.

These writers conclude that the commereial grade of sodium fluorid is the most satisfactory agent to use against lice. It kills lice in all stages including those hatching subsequent to its application. They apply the agent in two ways. When applied in the powdered form, the bird is held in a pan to catch superfluous powder. They apply one pinch on the head, one on the neck, two on the back, one on the breast, one below the vent, one on the tail, one on either thigh and one scattered on the inner side of each wing when spread. They recommend this method of application for newly hatched birds and sick birds exclusively. The action of the powdered sodium fluorid is comparatively slow, but it remains on the bird, and in four or five days all lice will disappear. The dust is somewhat irritating to the air passages and the operator should wear a dust protector over the mouth and nostrils. The powder causes a transitory effect only on the air passages of the treated birds. The method costs about $\$ 1.25$ per hundred fowls, calculating the man's time at 20 cents per hour.

Applying the agent in solution by dipping has the advantage in reducing cost of materials and is more rapidly done, without discomfort to the operator. Furthermore, the lice die very promptly after being touched by the fluid. The first requisite is a warm, sunny day so that the fowls will dry quickly. The solution is prepared in a tub in the proportion of three-fourths to one ounce of commercial sodium fluorid to each gallon of tepid water. In dipping the birds, the wings are held over the back with the left hand while the bird is submerged in the solution with the head out. The feathers are thoroughly ruffled with the right hand to secure penetration of the fluid to the skin all over the body. The head is ducked once or twice, the bird is drained a few seconds and is released. The work of dipping should be stopped sufficiently early in the afternoon to permit the 
birds to become dry befure night. By the dipping method the cost for material is 58 cents per hundred birds. For material and labor both, it is 71 cents.

Bishopp and Wood note that several other agents are effective in destroying lice, but are more expensive than sodium fluorid. Flowers of sulphur applied with a dust can in an amount of about 6 pounds per hundred fowls is effective in about four days. Dipping fowls in a solution of 1 ounce of laundry soap to a gallon of water, repeated in ten days is effective.

Herrick strongly recommends the Cornell or Lowry dusting powder made as follows: Two and one-half pounds of plaster of Paris is spread in a shallow pan or tray. One-fourth pint of crude carbolic acid is poured into a cup, and into this is poured three-fourths pint of gasoline. The mixture of acid and gasoline is poured over the plas-

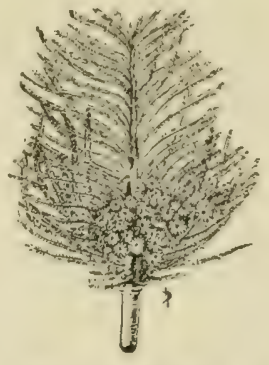

FIG. 41. Mass of body louse egrgs at t a ched to feather. Greatly enlarged. (Bish. opp and Wood) ter of Paris and thoroughly mixed. It is then rubbed through a wire window screen on a piece of paper and allowed to stand for from one and one-half to two hours or until thoroughly dry. It must not be placed near a flame or any heat. The powder should be kept in a closed can or jar, where it will retain its strength for a long

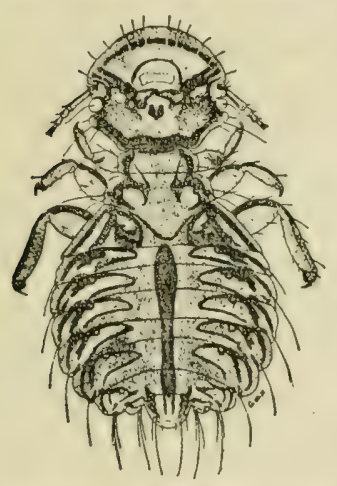

FIg. 42. Large hen louse. (Goniocotes abdominalis): Male, top view. Greatly enlarged. (Herrick) time. The powder is applied by means of an ordinary sifter or with the fingers. It is worked in among the feathers about the vent, in the fluff, and under the wings. Two and perhaps three applications should be made. A small pinch of the powder is sufficient for a fowl. The rooster should receive espeoial care in being kept free from lice, as he is an important factor in spreading the parasites.

The expense of this treatment including labor should not exceed 32 cents per hundred fowls.

The effectiveness of this method of dusting birds for lice has been called into question by several observers.

Lamson and Manter recommend the application of a dilution of either mereurial ointment or blue ointment. Mereurial ointment was considered cheaper when judged on the hasis 
of content of metallic mercury. The mercurial ointment is mixed with one or two parts of vaseline. One ounce is sufficient to treat about seventy-five hens. In the case of chickens it is desirable to place a piece of ointment the size of a pea, under the wings or around the vent. In older stock the best place is on the skin just beneath the vent. The treatment seems to remain effective for some time, so that lice hatching after the application are also killed. It is not clear that the use of mercurial ointment is cheaper or more effective than sodium fluorid. Bishopp and Wood find that when used as recommended, the mercurial product has little effect upon head and wing lice. Used in larger amount it is more expensive and injurious.

The dust bath constitutes the natural defense of fowls against lice and should be considered in connection with the measures against them. It is desirable that the box be located in the sunlight, and if covered, will prevent widespread dissemination of dust in the poultry house. The finer and lighter the material available, the better. Fine siftings from coal ashes is a desirable addition to the dust, as also are snuff, sulphur and dry slaked lime. The dust bath, while desirable, cannot be depended upon to the exclusion of more aggressive measures. It has only a temporary effect upon the numbers of lice on the fowls. Some birds seldom or never use the dust box.

In a flock infested with lice special care must be taken to free the sitting hen from these parasites and to protect young chicks in like manner.

\section{THE BIRD FIEA}

The common bird flea, Pulex avium, attacks a large number of species of birds including fowls and pigeons. The parasite is provided with piereing mouth parts with which it penetrates the skin and extracts blood. The irritation produced by the fleas causes pruritis and loss of rest.

Life history. The eggs are laid in hens' nests or on manure on the floor of the poultry house. These nits after a week or ten days hatch into white larvæ which are nearly a quarter of an inch in length. The larval stage lasts from ten days to three weeks, depend-

FIG. 43. Larra of hen flea, enlarged 20 times. (Railliet)

ing upon the temperature. The fully developed larva becomes secreted in some crevice and spins itself a cocoon. In this structure the larva develops into the adult stage during a period varying from 
ten to twenty-one days. Fleas are temporary rather than permanent parasites, and are nocturnal feeder's. During the day they hide in various protected places about the poultry house. Sitting hens suffer severely from their attacks.

Combating fleas. Repression of fleas is accomplished by the spraying necessary for combating mites. The use of whitewash containing a small percentage of kerosene oil destroys larve, fills up lurking places and drives adults away if it does not kill them. Wood sharings is a desirable material for making nests, as it is repellent to fleas. Simple construction of interior fixtures to facilitate removal and cleaning is as necessary in connection with combating fleas as in combating mites.

\section{CHIGOE}

A second true flea, the chigoe, Sarcopsylla gallinacea, causes injury in quite a different manner. The male penetrates the skin, while the female becomes fixed to the skin so it is not detached quickly. Fowls, ducks and various animals are subject to attack. The parasite is found in great numbers about the eyes and neck. It is widely distributed, having been reported as occurring in Ceylom, Florida, South Carolina, Italy, Turkestan and various portions of Africa.

\section{FLIES PARASITIC UPON PIGEONS}

I fly, Ornithomyia avicularia, is parasitic upon a number of wild birds and has been reported as occurring on pigeons in France. It moves between the feathers and draws blood from the host. Lynchiu maura is a closely related species parasitic upon pigeons in Sicily and Algeria. These flies are especially abundant upon squabs before the plumage has developed. Their attacks cause emaciation and sometimes also a fatal anemia. Sitting birds are disturbed by the parasites and make quick movements which may result in breaking the eggs. Sometimes the birds are so tormented that they abandon the dovecote. Sergent reports this fly as the secondary host of the blood parasite Homoproteus danielewskyi. L. capensis has been reported as parasitic upon pigeons in Teneriff.

\section{BED BUGS}

The enmmon bed bug Cimex (Acanthia) lectutaria nceasionally infests forls and pigeons at night for the purpose of sucking biood. During the daytime they inhabit eracks and sheltered places about the poultry house. 
NEST BUGS

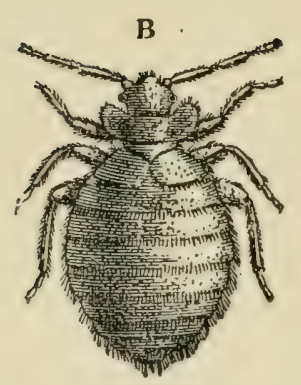

A

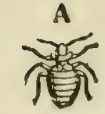

Fig. 44 Dovecote bug. $A$, natural size; $B$, enlarged. (Railliet)

The nest bug or dovecote bug Cimex (Acanthia) columbara is a species closely related to the bed bug and which also attacks pigeons and fowls. Sitting hens suffer severely. Both this species and bed bugs are held in check by the routine measures recommended in combating mites. C. inodorus occurs in Mexico, New Mexico and Texas.

\section{LARVAL BEETLES ATTACIING HENS AND PIGEONS}

Tenebrio molitor is a beetle the larve of which have been reported as attacking sitting hens and pigeons. Pigeons have been reported as subject to the attack of larvæ of Dermestes lardarius and other larvæ of the genus Necrophorus and genus Silpha. These coleopterous larve develop in the manure of the dovecote, attack the skin of the squabs on the neek and abdomen and even the superficial muscles. The wounds become infested with fly maggots and are frequently fatal. The presence of the larve may be prevented by cleanliness. Wounds may be treated with antiseptics.

\section{RED MITES}

Synonyms. Poultry mite, chicken mite, roost mite.

Hosts. Pigeons, fowls, turkeys, phensants, domesticated mammals and man are subject to attack by the parasites.

General nature. The common poultry mite, Dermanyssus gatlinc in general, infests birds only temporarily while they are on the roost. It is a blood sucker, and besides causing loss of blood, induces intense irritation.

Individual mites are barely visible to the rmaided eye, but owing to the habit of collecting in masses, they are easily detected, especially when engorged with blood and colored red. When not engorged, they are gray in color.

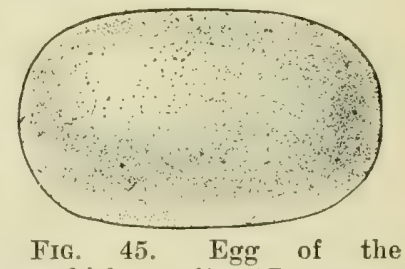
chicken mite (Dermanyssus gallina). Greatly enlarged. (Bishopp and Wood) 
The parasite is widely distributed throughout the world. Its depredations are more serious in warmer countries because of favorable elimatic conditions.

Habits. During the daytime the mites collect in cracks, or unler droppings and rubbish. They make their way at night to the birds on the roost where they cause annoyance while engorging with blood. Occasionally mites will attack hens on the nest during the daytime. When engorged, the mite leaves the bird. Some mites that fail to become engorged the same night they gain access to the bird, will remain on the host during the following day or so. Thus hens have been found to carry as many as a hundred mites during the daytime. Eggs are laid in the places sheltering the mites, and hatch in two days or more depending upon the temperature. The larvæ hatching from the eggs shed their skins in about two days and become nymphs. After several

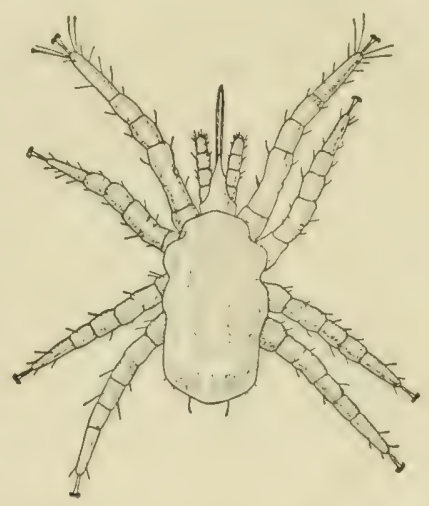

FIg. 46. First nymphal stage of the chicken mite, unfed. Greatly enlarged. (Bishopp and Wood) engorgements and molts, the parasite reaches maturity. The whole life cycle is complete in a week. The parasites are not active in cold weather. In the absence of poultry, mites will remain alive in infested houses for four or five months. Moisture seems to favor their longevity.

Injury caused by mites. Excessive mite infestation has been held responsible for increase in cost of maintenance, decrease of egg laying and general unthriftiness. Sitting hens have been driven by the irritation, to abandon the nest. The attack of the mites rarely leaves a trace on the skin.

Combating mites. In combating the pest, it is recommended that all interior fittings be removed so as to minimize the number of cracks in which mites could escape the action of the spray. If the house is of the older type, with built-in accessories such as perches and nests, everything should be torn out leaving merely the four bare walls. The whole house should be thoroughly swept and all rubbish removed. Kerosene oil, or kerosene emulsion constitute suitable agents for killing mites. One of these agents should be applied with a spray pump, particular care being taken to force the fluid into cracks. Since it is quite likely that some mites and eggs will 
escape being hit by the fluid, it is necessary to repeat the application in a week and perhaps a third time may be necessary.

Bishopp and Wood recommend crude petroleum as the most effective agent and one that retains its killing power for weeks. To

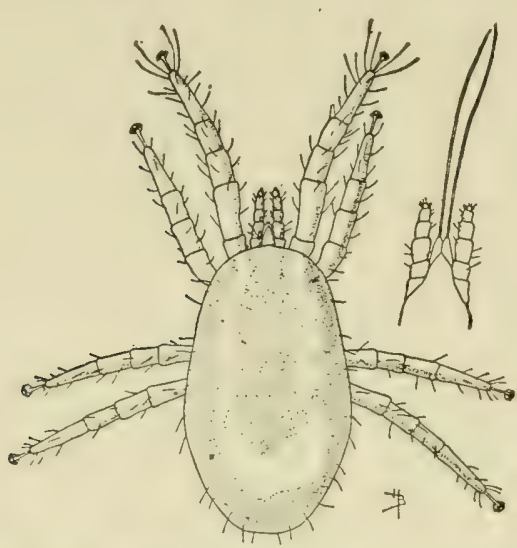

FIG. 47. Female chicken mite before feeding, greatly enlarged. Mouth parts at right highly magnified. (Bishopp and Wood.) improve its spraying quality it may be thinned with one part of kerosene to four parts of crude oil. They likewise find arsenical dip such as used for dipping cattle to be fairly satisfactory in killing mites when applied several times.

Repp recommends preparing kerosene emulsion as follows: One-half pound of laundry soap or whale-oil soap is shaved fine and dissolved in 1 gallon of water. The soap is best dissolved if the water is nearly or quite at the boiling point. When the soap is dissolved and the water is hot it is removed from the fire, 2 gallons of kerosene oil is added, and the mixture is agitated or churned violently until emulsion is formed. The best way to produce the emulsion is to pump the liquid back into the same container through the pump until the mixture becomes creamy. To make a ten per cent emulsion, 17 gallons of water are added to the 3 gallons of stock mixture; to make a fifteen per cent emulsion, $10^{1 / 3}$ gallons of water are added to the 3 gallons of stock mixture.

Subsequent to spraying with kerosene emulsion it is desirable to dust the entire interior of the houses with a mixture of air slaked lime, 3 parts and sulphur, 1 part.

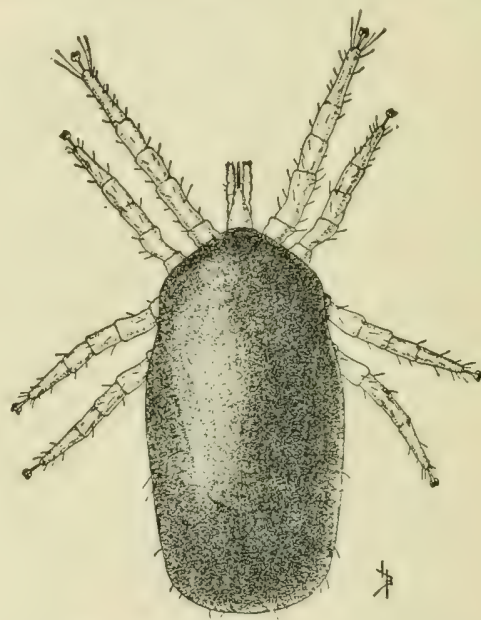

FIG. 48. Female chicken tick after feeding. Greatly enlarged. (Bishopp and Wood)

A further measure against mites consists in painting the perches 
with a mixture consisting of three parts of kerosene to one part of crude carbolic acid.

The presence of all sorts of rubbish affords lurking places for mites and decaying matter such as broken eggs attracts them particularly.

When fowls are moved to fresh quarters from infested quarters it is desirable that they be left for three days in a pen so that all mites will have opportunity to leave the birds.

Mites are disseminated not only by live birds bearing them, but by all such articles as crates and other objects that have been in poultry houses. The introduction of mites by pigeons or sparrows is quite possible.

Infestation of man. People in close contact with infested hen roosts are liable to be attacked by the mites. They cause an ephemeral itching lesion on the back of the hand and other exposed portions of the body. However, they may affect any portion of the body.

Occurrence on horses. When poultry roosting quarters are near or in the stable, horses are subject to infestation. They cause severe pruritis during the night. The mites cause the formation of small vesicles single and grouped together. These vesicles are rubbed off, accompanied by depilation, but the areas soon become covered with new epithelium. This gives the skin a characteristic flea-bitten appearance. To these changes are added others resulting from excessive rubbing.

Mites in the external ear. Mites have been observed in the outer ear of chickens and ducks. In the case of the latter, unusual movements of the head occurred. The mites were considered by the observer to be $D$. gallince but Neumann expresses doubt concerning the identification.

\section{HARVEST MITES}

Harvest mites are first stage larvæ of certain species of the genus Trombidium. They are commonly known as chiggers, a name which is evidently a corruption of the term chigoe, used to designate a species of flea. The harvest mites attack a wide variety of hosts, including poultry. They attach themselves in groups to the skin beneath the wings and on the breast and neck. Their presence causes areas of serere irritation and suppuration. Young chickens are most susceptible and their death has been attributed to the result of the presence of the parasites.

Mites oceur in a wide variety of places such as in damp locations 
along the borders of streams, in trees and shrubbery and on the borders of forests.

In the United States they are most numerous in the South but are well distributed in Kansas, Indiana and Iowa. In England and Scotland they are known popularly as gooseberry bugs.

Chickens should, so far as possible, be kept from ranging in areas known to be infested. Dusting the feathers with sulphur is useful in keeping the mites off. The application of sulphur ointment or kerosene and lard for killing the mites on the skin is recommended by Bishopp and Wood. Eroded areas may be treated antiseptically.

\section{SCALY LEG OR FUOT MANGE}

The mite designated Cnemiodocoptes mutans (Sarcoptes mutans)

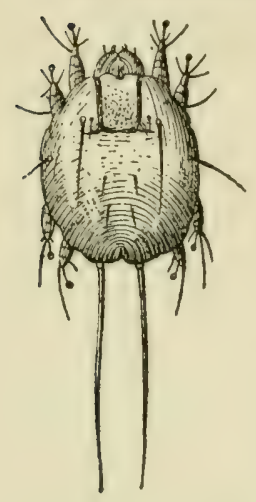

FIG. 49. Cnemiodocoptes (Sarcoptes) mutans. Male, dorsal aspect enlarged 100 times. (Neumann) affects the legs of fowls below the tarsal joint. Other domesticated birds are affected more rarely. The first evidence of infestation consists of the appearance of grayish white areas which gradually coalesce and thicken. The parasites burrow in the skin and set up cellular proliferation with exudation. This thickens the skin and canses the natural scales of the leg and toes to protrude. In an advanced ease the skin is covered with thick grayish white crusts which appreciably increase the size of the leg and toes. The enlargement of the leg interferes with the motion of the joints. The skin is inflamed and raw. Examination of some of the crusts will reveal the presence of mites. Itching is present as indicated by the pecking of the lesion by the bird. In extremely advanced cases the bird may become lame, arthritis may develop, toes may be lost, and the bird may squat continuously. The infestation may be accompanied by loss of condition and even cachexia terminated by death.

Treatment. The legs of the affected bird should be soaked in warm soapy water and be thoroughly cleaned

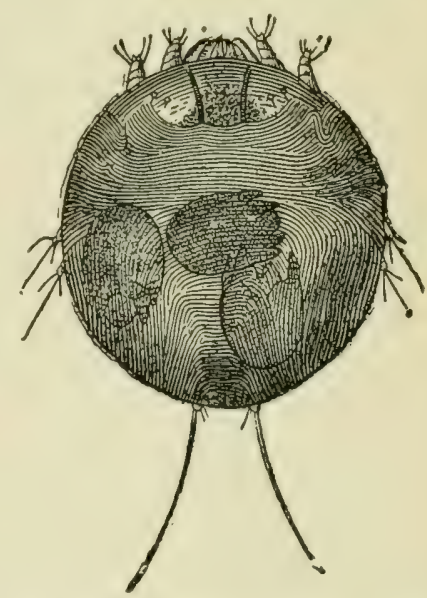

FIG. 50. Cnemiodocoptes (Sarcoptes) mutans, ovigerous female, dorsal aspect, enlarged 100 times. (Railliet) 
by serubbing with a brush. The most effective remedy consists of the application of an ointment consisting of one part of caraway oil and four parts of lard or of white vaseline. Another remedy consists of sulphur ointment made of 1 part of sulphur and nine parts of lard. Crude petroleum is effective. Plain kerosene oil sometimes employed is somewhat effective but will become smeared on the thighs as soon as the bird perches, and will blister the skin. The application of any remedial agent should be repeated.

Prophylaxis. Removal of infected birds from the flock, and general disinfection should be carried out immediately following the discovery of the disease.

\section{DEPLUMING SCABIES}

The mite designated Cnemiodocoptes (Sarcoptes) lavis may occasion loss of

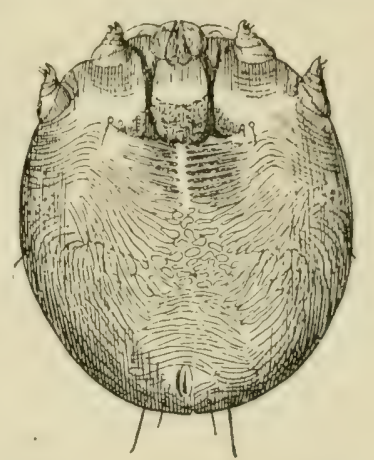

Fig. 51. Sarcoptes mutans. Ovigerous female. Dorsal view. Enlarged 100 times. (Railliet)

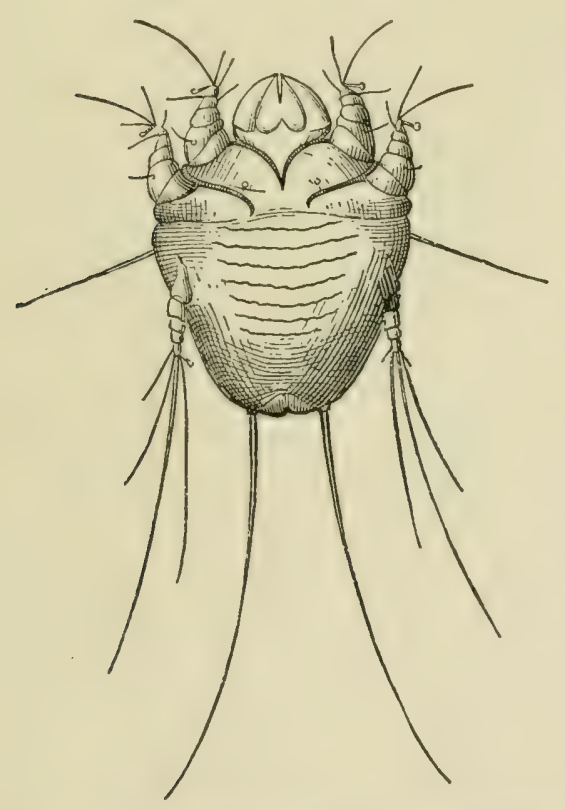

'Fig. 52. Cnemiodocoptes (Sarcoptes) levis, larva, ventral aspect, enlarged 200 times. (Railliet) feathers in fowls, pigeons and pheasants during the spring and summer. It infests the portion of the quill that lies beneath the surface of the skin. The affection usually appears first on some spot on the body, and progresses gradually to the neck, thighs and head, or the head and neck may be affected first. The frequency with which the back is first affected suggests that treading may be the principal means of dissemination. The large feathers of the wing and tail are not generally involved.

The feathers are either shed entire, or the quills break off close to the level of the skin. Sometimes the stumps are cracked and are surrounded by epidermal scales. 
The mites may be observed on the stumps of broken off quills or of feathers drawn from the border of the bald area. The irritation set up by the mites at the root of the feathers often causes the bird to pluck its feathers, or even sets up the habit of pulling the feathers of other birds.

The skin is generally normal in appearance but occasionally appears red and nodular. In severe cases, egg laying may be interfered with, and the birds become emaciated and die.

Treatment. Treatment consists in the repeated application of essentially the same remedies as recommended for scaly leg, such as sulphur ointment. Salmon recommends two liquid preparations, a solution of balsam of Peru in alcohol (1:3) or a mixture of creolin, 1 dram; glycerine, 2 ounces; alcohol and water, 1/2 ounce each. Schleg's solution has been recommended for treatment. This consists of white arsenic and potassium carbonate each 1 dram, 3 pints of water and 3 pints of vinegar.

\section{OTHER MITES AFFECTING SKIN AND FEATHERS}

The mites Epidermoptes bilobatus and Epidermoptes bifurcatus have been found associated with a form of scabies affecting the skin. The beginning of the infestation is indicated by the appearance of fine yellowish scales which eventually develop into thickened brownish crusts. The parasite in question is frequently found on birds without the presence of lesions, so their presence in a lesion does not necessarily indicate that they are the cause. Treatment is similar to that suggested for scaly leg and depluming scabies.

Other mites parasitic upon the skin and feathers and of less economic importance are listed by Neumann as follows: On the fowl, Pterolichus obtusus, Dermaglyphus minor, D. elongatus, Megninia cubitalis and M. columbe; on the turkey, Freyana chanayi, $D$. minor and II. cubitalis; on the guinea-fowl, D. minor and $D$. varians; on the pheasant, $P$. obtusus, $P$. uncinatus, $M$. cubitalis and $M I$. ginglymura; on the peafowl, $M$. cubitalis and $M$. ginglymura; on the pigeon, Falculifer rostratus, $F$. cornutus, M. columbe, Analges bifidus and Pterophagus strictus; on the goose and swan, F. anserina; on the duck, $F$. anatina and $M_{i}^{\top}$. velata.

\section{OTHER PARASITIC MITES}

Mites parasitic in parts of the body other than the skin, occur in the nasal cavities, air sacs and connective tissue. These are discussed in Chapter XVI. 
TIIE FOIVL TICK

Designations. Argas miniatus (A. Americanus): In various scetions of the country the tick is designated by such popular names as tampan, blue bug or blood sucker. It has been referred to in the literature as the miana bug, from whence its specifie name is derived.

Hosts. Besides infesting fowls, it attacks turkeys, gecse, pigeons, ostriches and canaries.

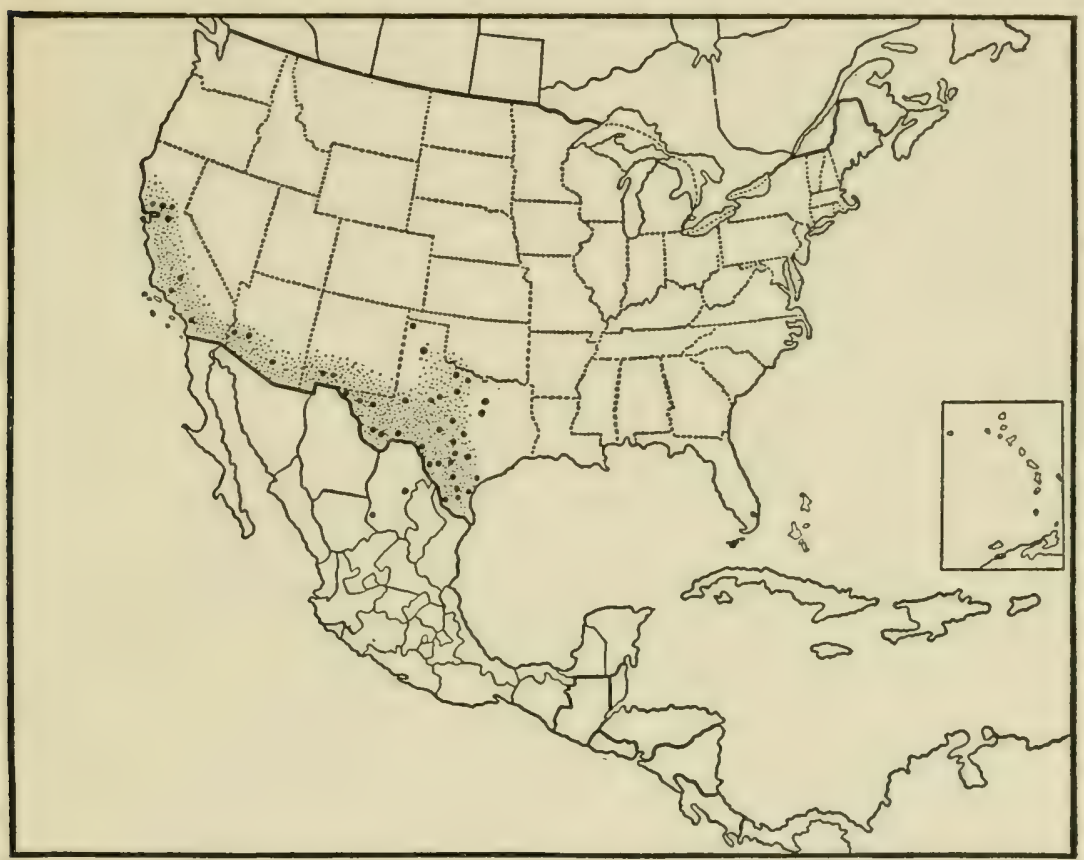

FIG. 53. Distribution of the fowl tick (Argas miniatus) in the United States. The large dots indicate localities where this tick has been collected by the Bureau of Entomology. The small dots show the normal distribution of the species within the United States. (Bishopp, Bureau of Entomology, U. S. Dept. Agr.)

Injury produced. Bishopp summarizes the ways in which harm is done as follows: (1) Through death which may oceur in ponltry of all ages; (2) by the lowering of the vitality of the fowl so as to make it readily susceptible to disease; (3) by greatly reducing egg production; (4) by stunting the growth of chickens; and (5) by disturbing sitting hens. He further observes that death may be caused by gross infestation which drains the fowl of blond and 
produces intense irritation. This occurs when fowls are placed in coops which have not been oceupied for months and which harbor hungry ticks. The infested birds appear paralyzed, the wings droop, the feathers are ruffled and appetite is lost. Death may oceur within a few days after such heavy infestation, but if removed from the ticks, the birds will recover.

The discussion of the subject of harm induced by tick infestation alone, is complicated by the fact that in most countries ticks are the carriers of spirochetosis, which is described on p. 135. In the absence of positive information as to whether or not the fowl ticks of this country carry spirochetosis, it is not possible to state just how much injury is done by ticks alone and how much by a disease transmitted by them.

Distribution. The tick oceurs in the western half of Texas and in a zone extending westward along the southern part of New Mexico, Arizona and California. In the latter state the area extends northward along the western half of the state to a point slightly north of San Francisco. The area involved is indicated in Fig. 53. The distribution of the tick is thus seen to be confined to the warm portions of the arid and semi arid regions. In Texas it does not extend into areas where the annual rainfall exceeds 30 inches, which apparently prevents its extension into the eastern part of the state or eastward on the Gulf coast. Ticks may occasionally be shipped to northern states and find favorable conditions for a limited period. Elsewhere it occurs in Persia, Australia, Africa, South America, the West Indies, Mexico, Panama, India, Southern Russia and Roumania.

Life history of the tick. The habits of the fowl ticks resemble those of bed bugs in that they are night feeders and spend the day in cracks and other protected places. Thus considerable injury may be inflicted to poultry without the knowledge of the owner.

Eggs are deposited in secluded places and in the summer, hatch in from 10 to 15 days. In the winter the hatching period may extend over three months. The young seed ticks soon make their way to a fowl and attach themselves to the skin of the breast, thighs, or elsewhere and engorge with blood. This period of feeding, during which the ticks are constantly attached to the fowl occupies from $31 / 2$ to 10 days. When it is completed the ticks drop from the host. Before feeding they are light gray in color but after feeding they become dark blue, and are about one-tenth of an inch in length.

After 4 to 9 days the seed ticks undergo a molt and develop into 

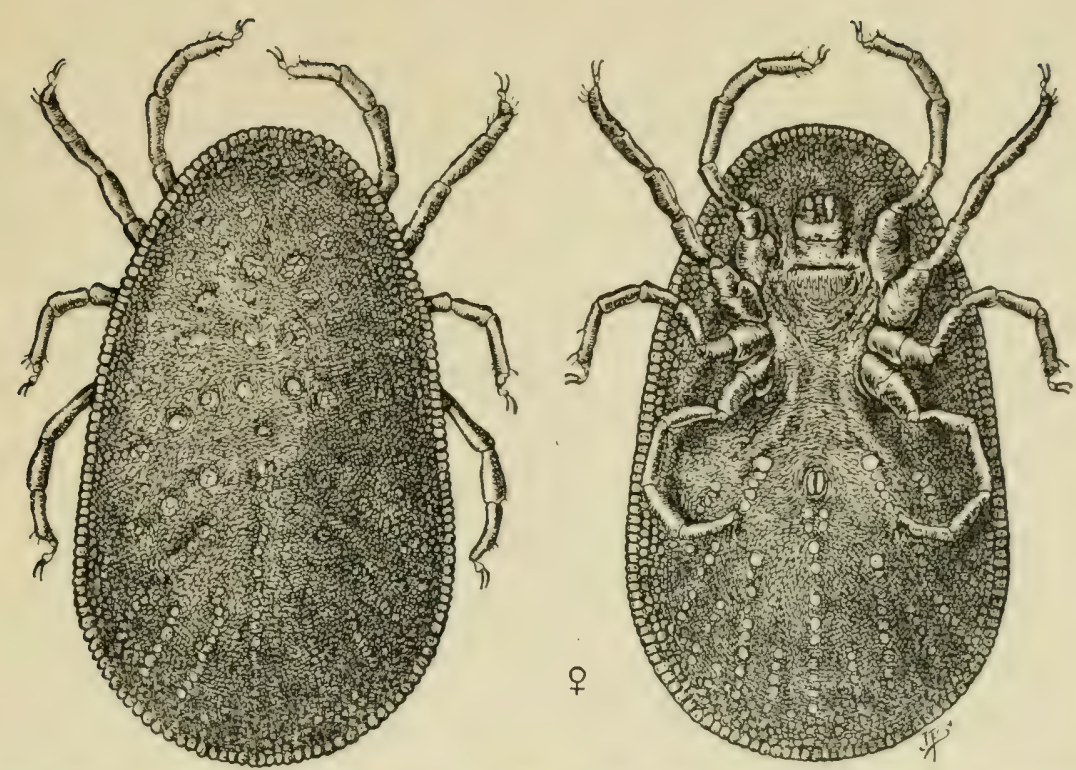

Fig. 54. Female fowl tick, as seen from above and beneath. Greatly enlarged. (Bishopp)

nymphs. The feeding habit of nymphs is different in that they remain attached to the fowl for but a short time, at night. Nymphs molt two or three times, alternating with feeding periods before they become adults. The adult female tick is shown in Fig. 54.

Contrary to the habits of many ticks the adult female does not die after depositing eggs. She may alternate feeding periods on the fowl with egg laying for three, four, or even seven times.

Ticks display a remarkable ability to exist for long periods without food. Seed ticks have lived for $5 \frac{1 / 2}{2}$ months without food, those in the first nymphal stage for nine months, those in the second nymphal stage for 15 months, and adults for $2 \frac{1}{2}$ years.

Combating ticks. The difficulties associated with eradicating ticks frcm a hen house are so great that in case the building is of little value, burning the structure would save time and trouble. Under conditions forbidding the employment of this extreme and effective measure, various substances may be sprayed on the woodwork and in the cracks. Crude Beaumont petroleum, carbolized whitewash, boiling water, strong kerosene emulsion and ririous creosote disinfectants have been recommended. Hot tar eliminates cracks and imprisons ticks. Ticks are very resistant to substances such as insect powder, kerosene and even fumigation with hydro- 
cyanic acid gas. No one application of any substance should be depended upon to kill all ticks, because of their ability to secrete themselves, and because eggs secreted in cracks may escape destruction and hatch.

In an infested flock, control measures must not be limited to the buildings, because first stage larvæ remain attached to birds for days. To deal with these it may be quite sufficient to isolate the birds in other than the usual quarters, until the crop of larvæ become engorged and drop off. An application of kerosene and lard to the breast and underneath the wings will destroy larvæ. Dipping the birds in a 10 per cent solution of one of the coal tar dips is effective, but in general dipping is not recommended.

Since ticks must crawl to the birds and do so only at night, suitable isolation of the roosting perches is a valuable measure in protecting birds. The construction of the roosting poles should be such as not to afford harboring places. The poles may be suspended from the ceiling by rods passing through cans of kerosene or supported from beneath by a similar device. Such measures are eftective until the fluid becomes bridged over with dust or other material.

Tick-proof construction. The secretive habits and resistance of ticks are such that the menace occasioned by them can effectively be met in the long run only by providing special building construction. A poultry house built of painted corrugated iron or of galvanized iron over a framework of piping solves the tick problem. Cracks are reduced to a minimum and the extreme heat attained by the building during the daytime, is destructive to ticks. Furthermore, ticks may be destroyed by building a fire inside the building, or by the use of a gasoline torch. Tick-proof perches and nests may be secured by setting the supports in vessels of kerosene.

If it is not possible to provide all-metal construction, the wooden building should be put up with reference to avoiding cracks. In any event a shingle roof should be avoided.

\section{THE PIGEON TICK}

The pigeon tick (Argas reflexus) occurring in Europe is a species closely related to the fowl tick. It is somewhat rare in England, Germany and other northern countries. Its habits are similar to the fowl tick. This species is a serious pest of pigeons, while fowls, ducks and geese are also attacked. 


\section{REFERENCES}

1. Bishopp. The forl tick. U. S. Dep. Agr. Bureau Entomol. Circ. 170.

2. Bishopp and Wood. Mites and lice on poultry. U. S. Dep. Agr. Farmers' Bull. 801.

3. Fuller. A fowl infesting tick. Agr. Gaz. N. S. Wales, Vol. 7, 1896, p. 590 .

4. Haidub. Die Fussräude des Geflugels. Inang. Diss. Giessen.

5. Hassall. Note on the chicken tick. U. S. Dep. Agr. Bureau Animal Indust. 16th Ann. Rept., 1899, p. 496.

6. Herrick. The chicken mite. Miss. Agr. Exp. Sta. Bull. 78.

7. Herrick. Some external parasite of poultry. Cornell University Agr. Exp. Sta. Bull. 359.

8. Lamson and Manter. Some lice and mites of the hen. Connecticut (Storrs) Agr. Exp. Sta. Bull. 86.

9. Laurie. The poultry tick. Dep. Agr. So. Australia, Bull. 74, 1912.

10. Lounsbury. The fowl tick. Agr. Jour. Cape of Good Hope, Vol. 23, 1903, p. 261.

11. Repp. The chicken mite. Iowa Agr. Exp. Sta. Bull. 69.

12. Robertson. Fowl tick experiments. J. Dep. Agr. West Australia, Vol. 12, 1905, p. 561. 


\title{
CHAPTER XVIII
}

\section{INFECTIOUS DISEASES AND PARASITES OF THE OSTRICH}

\author{
ANTHRAX IN THE OSTRICH
}

Characterization. Anthrax of the ostrich is an acute febrile infection caused by Bacterium anthracis.

History. The disease was first reported by Henning in the Cape of Good Hope in 1894, and later by Robertson. Theiler in 1912 also has described the disease.

Etiology. Anthrax of mammals and of the ostrich is identical, the causative agent being $B$. anthracis.

Pathogenicity. The disease is transmissible from ostrich to ostrich by incenlation with heart blood. It is also communicated from the ostrich to the sheep and goat by inoculation. A strain of anthrax occurring originally in cattle was inoculated into a sheep, with fatal result, and blood of this animal when inoculated into an ostrich caused death from the same disease.

The susceptibility of man to anthrax constitutes an important reason for care in conducting autopsies.

Symptoms. According to Theiler anthrax occurs in the ostrich in two forms: the peracute or apoplectic form and as anthrax fever. The peracute form attracts most notice for it invariably ends in death. In natural cases the birds are generally found dead in the morning, or in a moribund condition, comatose, paralyzed and apparently insensible. A bird may present the picture of health one moment and be dead of the disease a few minutes later. In such a sudden attack, the feather's become erect and the whole body is shaken with tremors. The bird staggers, lies down on the side and kicks convulsively, while the neck twists and untwists spasmodically.

More chronic cases have been designated anthrax fever. The birds show symptoms of a general illness and do not feed well for a few days. The attack results in death, or more commonly in recovery.

Morbid anatomy. In the apoplectic cases the carcass is frequently in the best of condition and rigor mortis usually is absent. The blood escaping from the distended cutaneous and subcutaneous veins is usually dark in color and coagulates badly. 
A smaller or greater amount of liquid is present in the pleuroabdominal cavity and is occasionally colored red.

Petechire, echymoses or larger diffuse patehes of hemorrhagic infiltration are observed on the ribs, on the pericardium, the peritoneum and mesentery. Gelatinous infiltration of tissues also is observed.

The pericardial fluid may be increased in amount. Hemorrhages and extensive hemorrhagic infiltrations oceur on the endocardium and epicardium. The blood in general is black in color, tarry in consistency and is either coagulated not at all or only partially.

The lungs, proventriculus and gizzard are usually normal in appearance.

The small intestines usually show more or less intense infiltration with blood throughout the mucosa, which structure shows a uniform reddish discoloration. In some cases the hyperemia is irregularly distributed in patches and streaks throughout the length of the intestines. The condition is visible from the serous surface of the intestine. The acute inflammation may be found to be more pronounced in the colon where the mucous membrane is yet thicker. The surface of the mucosa is corroded, the submucosa is gelatinous and studded with hemorrhages, or softened and enntaining neerotic patches. The contents of the colon may include blood.

The liver shows acute congestion, is enlarged and bluish in color. Blood escapes freely when the liver is incised.

The spleen sometimes is normal in size and condition. However, usually it is enlarged and dark colored. The kidneys may be normal or congested, dark in color and friable in texture.

Treatment. Theiler recommends carbolic acid diluted in water, in doses varying from one dram for a three months old bird to four drams for a two year old bird.

Prevention. The preventive measures of isolation of sick and disinfection are very similar to those indieated in outbreaks of the disease among mammals. Carcasses preferably should be burned without opening or if this is impossible, should be buried in quicklime at a good depth. All blood spilled at an autopsy as well as all excreta of infected birds should be burned. Abandonment of infected runs is desirable, but if this is impossible, the use of the Pasteur type of anthrax vaccine may be undertaken.

Anthrax infection of soil constitutes practically a permanent eondition. Opportunity for direct infection from the soil by contamination of feed, or by wounds will vary with seasonal conditions of 
moisture. Inundations and extension of irrigation systems may spread the infection to new foci. On some of the infected farms cited by Theiler the infection seems to have first attacked cattle. In one case the infection had been introduced by cattle. In other outbreaks, ostriches died occasionally, while horses and cattle escaped.

Every precaution should be taken to prevent the exposure of anthrax-infected blood to the air. Under such conditions spores form, which are capable of remaining alive in the soil for an indefinite period.

\section{ASPERGILLOSIS IN ADULT OSTRICHES}

Walker has observed that in adult birds, marasmus is the only sign of aspergillus infection. When the lungs are affected, respiration is accelerated and the beak is kept partly open. There is pronounced loss of condition when the liver is involved. The disease usually runs a sub-acute or chronic course.

The lesions are found to be more or less generalized, the liver and respiratory tracts being most generally involved. Degenerative changes in the tubercles and destructive changes in the surrounding tissues are marked. The tubercles may exhibit the various changes in the evolution of a tubercle, such as miliary granulations, caseous degeneration and fibrous transformation. The lesions in the liver usually consist of extensive caseous areas caused by the degeneration of agglomerated tubereles and by necrosis of adjoining liver tissue.

Aspergillosis in the adult ostrich as reported by Jowett may present a condition very closely simulating miliary tuberculosis of the lungs. The lungs are consolidated, dark red in color, and are filled with yellowish-white miliary tubercles about the size of a pinhead, which stand out prominently against the background of congested and hepatized lung tissue. The tubercles are not readily removed for the periphery of each is united to others. They are fibrous and shot-like in consistency. They show no evidence of caseation nor calcification. There is slight thickening of the pleura, and a few nodules are present, but no lesions are observed in the mucosa of the trachea and bronchi.

Examination for tubercle bacilli yields negative results, but microscopic examination of crushed nodules in caustic potash reveals the true nature of the infection.

Study of sectioned specimens shows that each nodule is composed of: (1) an unstained or very faintly stained central area, (2) sur- 
rounding this an intensely stained peripheral zone, and (3), surrounding all, a zone in which the air cells show inflammatory changes but are still pervious. The center of the nodule has undergone coagulation necrosis and contains filaments and free spores. The next zone contains leucocytes, endothelioid or epithelivid cells together with many filaments.

The fungus undoubtedly is an aspergillus, and quite likely fumigatus, although no cultures were made.

The source of infection was regarded as moldy food.

Archibald has reported upon aspergillosis in the Sudan ostrich, in which quite different lesions were observed. On opening up the respiratory passage a peculiar lesion of the bronchioles is observed. The upper portion of a bronchus is lined by dark granular masses which project into its lumen. The masses are friable, but are closely adherent to the wall. The portion of a bronchiole next to the lung shows several "plaques" raised from the surface. These are grayish in color with a nodular center and vary in size from a millet seed to a pea. Most of them are discrete, but a few have coalesced. Their surface is thrown into ridges and depressions. Microscopic examination reveals the nature of the infection.

Aspergillosis in other birds is discussed in Chapter $\mathrm{X}$.

\section{ASPERGILLOSIS IN OSTRICH CHICKS}

Synonyms. Yellow liver, chick fever.

Characterization. Aspergillosis in the ostrich chick is a highly fatal affection caused by aspergillus fungi, of which Aspergillus fumigatus is the most common.

History. The disease was mentioned by Douglas in 1881 who observed that it was so destruetive that on some farms in Cape Province every chick died.

We are indebted to Walker for making a useful experimental study of the various aspects of the affection, which has elucidated the problems of etiology and prophylaxis.

Symptoms. In young chicks a few days old the first symptom is a disinclination to feed. The bird may go through the motions of picking, without swallowing food. The chick appears lull, weak, the eres are half closed, it moves about slowly or stands, and eccasionally utters a plaintive note. The neck is commonly flexed and the head is lowered and kept close to the body. The abdomen feels less tense and full than that of a healthy chick. The respiration is 
sometimes accelerated. The beak may be kept continually open, or only kept open during a long inspiration. The temperature is irregular, there being a variation of $3^{\circ}$ to $5^{\circ} \mathrm{F}$. between the morning and evening temperatures. In healthy birds the temperature varies about two degrees, that is between $102^{\circ}$ and $104^{\circ}$ F. Sub-normal temperature precedes death.

In some cases whitish nodules, about the size of a pin head may be detected on the buccal mucous membranes and the epiglottis.

Destructive outbreaks of the disease occur among chicks varying in age from a few days to 3 or 4 weeks. It is much more prevalent among artificially reared chicks than among those raised naturally.

Death occurs within a few days after the appearance of symptoms and it is seldom that an affected bird recovers.

Morbid anatomy. In young chicks naturally infected the lesions are chiefly confined to the lungs and air sacs. Less commonly nodules are observed on the buccal mucosa. A close examination of the lungs will usually reveal more or less rounded nodules averaging $1.2 \mathrm{~mm}$. in diameter, and yellowish white in color. Rarely caseous foci or pneumonic patches are observed in the lungs. The lesions on the walls of the air saes consist of either small tubercles or fibrinous exudate.

The tubercles are composed of epithelioid cells, lencocytes and some eosinophile cells, mixed with mycelium. The duration of the disease in chicks is so short that caseous degeneration and fibrous transformation of the tubercles do not usually occur.

Etiology. The ostrich is susceptible to several species of aspergillus fungi but the one most pathogenic, and more commonly encountered is Aspergillus fumigatus. A. asper also is pathogenic for the ostrich. The fungi are widely distributed among substances of importance in connection with the artificial rearing of ostriches. Straw may be naturally infected as also lucerne (alfalfa), and the soil upon which lucerne is growing. The feces of an ostrich fed upon lucerne are infective.

Pathogenicity. Intravenous injection of ostrich chicks with spores produces a more or less generalized infection in which the lungs and liver particularly contain tubercles. Death oceurs in from 2 to 7 days. Inhalation of spores produces lesions in the lungs consisting of dirty white colored nodules isolated or agglomerated, and lesions in the air sacs consisting of nodules. Chicks varying in age from a few days to 31 days die in from one to ten days after the 
introduction of spores into the trachea. Ingestion of cultures administered in a gelatine capsule causes the formation of tubereles, but no marked intestinal changes. The chicks die in from 5 to 18 days.

Dosing with the fungus in a gelatin capsule proluced no effect upon one bird 41 days old, and upon one adult.

Transmission. Chicks are susceptible to infection by both inhalation and ingestion. Opportunity for infection exists even before hatching, for the aspergillus fungus often infects the air sac of the egg. It is capable of penetrating unbroken shell and can thus pass from infected to non-infected eggs. Thus ahicks become infected before or during hatching, and the incubators become contaminated, constituting a menace to later hatches. Infected straw used as bedding in chicks' sleeping boxes is a serious source of infection.

Diagnosis. Aspergillosis may be identified by microscopic examination of nodules after treatment with potassium hydrate, in which case the mycelium and fruiting heads may be recognized. Nodules planted on potato and incubated for $2 \pm$ to 36 hours at $35^{\circ} \mathrm{C}$. produce small whitish growths visible to the naked eye. After incubation for a day more the growth appears bluish in color and still later turns dark bluish green.

Control. Prophylaxis of aspergillosis concerns securing non-infected eggs for incubating, non-infected incubators, non-infected bedding and safe feed for the chicks.

Treatment. No medicinal treatment is available and all efforts against the disease must be centered upon prevention.

\section{PARALYSIS OF THE OSTRICH}

\section{Synomym. Leg-weakness.}

History. In 1900 Marx observed the disease among Sudan ostriches in the Frankfurt Zoological gardens. Robertson in 1909 described a form of paralysis occurring among ostriches near Cape Town. The disease is of considerable economic importance and seems to be becoming more common.

Symptoms. The disease is abrupt in onset. There is sudden inability to rise, the interference apparently being in the toes, which are flexed on themselves at right angles. The birds make futile and persistent efforts to rise, and in consequence stageer along on the horek joints. After a few days, effort to rise is abandoned and the paraly- 
sis eventually involves the wings and neck. Appetite remains good and there is no evidence of pain. Marx noted the presence of marked lachrymation in every case. Affected birds have been observed to live for seven months and no instance of complete recovery has been noted. Young birds are most commonly affected, but the disease may be encountered among those of any age. The disease occurs at irregular intervals in certain areas.

Morbid anatomy. The one constant lesion as observed by Robertson is the congestion or inflammation of the duodenum and the reddened appearance of the mucosa, accompanied by a deposit of exudate thereon. This lesion is present in some degree in all cases, whether early or of long standing. The sub-mucous layer of the intestine is thickened and infiltrated with a clear straw colored fluid.

Marx observed more or less peritonitis and purulent foci in the spleen in each case. In some cases there was enteritis with necrotic patches on the mucosa.

Bacteriology. Robertson obtained from the inflamed mucosa, a short rod which does not stain by the Gram method. It grows fairly well on the common culture media under aerobic or anaerobic conditions. There is copious white growth on plain agar, glycerine agar, agar, gelatin and malachite green agar. On the latter the color is discharged along the needle track. Gas is evolved in sugar agar stab cultures. Growth oceurs in plain bouillon, glycerine bouillon and sugar bouillon causing turbidity and foul odor. A brownish colored raised growth cecurs on potato. Milk is not coagulated nor does liquefaction occur in gelatin. In natural cases, besides the organism in question, Stuphylococcus pyogenes aureus almost invariably is present in cultures from the sub-mucosa of the duodenum.

Marx isolated a non-motile, non-spore bearing, Gram negative, oval, bipolar staining rod, which is killed by exposure to $68^{\circ} \mathrm{C}$. for an hour. It grows readily on the ordinary culture media. Coagulation of milk occurs, growth on gelatin resembles that of B. coli, and a brownish viscid layer is produced on potato. Glucose is fermented and no indol is produced. After 24 hours on agar there appear grayish white colonies, iridescent by transmitted light which eventually develop into a moist greenish white layer. Bouillon becomes turbid in a few hour's and a deposit of sediment forms in the tube. Marx regards the organism as belonging to the hemorrhagic septicemia group.

Pathogenicity. Subcutaneous injection of 25 c.c. of a 24 hour culture of Robertson's organism into a cock ostrich resulted in the 
appearance of the typical symptoms in seven days, followed by death in about a month. Similar results were obtained with a number of other ostriches. The organism injected can be recovered from the lesions induced in the duodenum. Robertson regards the symptoms as indicative of a poisoning. He holds the opinion that the organisms do not invade the general tissues, but remain localized as in the case of diphtheria and tetanus. The poison is elaborated by the organism in the intestine and carried to the spinal cord and thus induces paralysis.

The organism isolated by Marx was found to be pathogenic for the gray mouse, pigeon, and various small birds. Guinea pigs and rabbits were insusceptible.

Control. The common source of infection is regarded as contaminated food and water. Robertson advises that infected birds be killed, and that the birds be shifted to bare veld and fed by hand with cut green crops. The continuous breeding of birds on one spot seems to be a factor contributing to the occurrence of the disease.

Treatment. Gray suggests the trial of intestinal disinfection with cyllin, beta-naphthol, sodium salicylate, quinine or bismuth.

WIREWORMS IN OSTRICHES

The wireworm, a nematode designated Trichostrongylus douglasi, symonym Strongylus douglasi, is a parasite infesting the mucosa and glands of the proventriculus of the ostrich. Adult birds, if well fed tolerate the parasite comparatively well but ostrich chicks frequently succumb. This parasite occasions great losses in the ostrich farming industry of South Africa.

Comparatively little was known of the life history of the wireworm and remedial measures until the publication of the work of Theiler and Robertson from which we have drawn largely in discussing the problem.

Life cycle of the wireworm. The parasite undergoes four molts corresponding to four different stages. Two stages are encountered in the life cycle outside the host and two inside the host. The crcle may thus be divided into a parasitic and a non-parasitic period.

The wireworm in its non-parasitic stages is free living. Its future propagation and dissemination depend upon gaining aceess to the body of an ostrich. This phase of its life history is of great practical interest in connection with the application of measures to prevent the infection of clean birds. 
The egg of the wireworm is expelled from the body in the feces of the bird in about three days after expulsion from the worm. Examination of feces of a number of birds has shown that one dropping of feces may contain from 24,000 to 600,000 eggs. A bird isolated under conditions preventing it from becoming reinfected has continued to discharge worm eggs for a period of nine months without showing decrease in number. The limit to the time a bird will discharge eggs has not been determined. The worm eggs in fresh droppings are non-infective. If fed to an ostrich they will be passed in the feces so long as they are fed, but autopsy of the bird after an interval will show that no worms are present.

The worm eggs hatch only outside the body of the bird and the larvæ undergo four molts before reaching maturity. Hatching and the development of the larvæ are sharply controlled by temperature and other conditions. At a temperature of $98^{\circ} \mathrm{F}$. ova hatch and the larvæ reach maturity. At $100^{\circ}$ to $101^{\circ} \mathrm{F}$. hatching occurs, but only the older larvæ reach maturity. The same is true of a temperature range of $102-102.5^{\circ} \mathrm{F}$. A temperature of $104^{\circ} \mathrm{F}$. does not permit the development of larve. Ova and larve in the first stage may be kept dry at room temperature for two years and develop to maturity when moistened. Ova and first stage larva cease development when dried over calcium chloride, but remain alive for at least three years. Eggs retain their vitality in water but cannot reach maturity at a depth of two or three inches. The vitality of eggs is not destroyed by a temperature of $32^{\circ}$ to $46^{\circ} \mathrm{F}$. for three months. Droppings kept under natural outdonr conditions for fifteen months contain live mature larvæ and first stage larvæ.

Larvæ in the first stage and immature larvæ of the second stage do not develop in the stomach of the ostrich. Mature second stage larre alone are capable of becoming established there. Liquid is undoubtedly a suitable medium for these larve and they are very active in such an environment. A temperature below $38^{\circ} \mathrm{F}$. checks their movement under these conditions. When droppings and second stage (mature) larvæ are left exposed on soil where grass is growing, the larva crawl to the tip of the grass blades where they are more numerous than on the portion below. They have been observed to remain alive on green grass for six months and for two months more after the grass dried up although in diminished number. On a second crop of grass, larvæ were observed at a height never exceeding three inches. From one infection of the soil larvæ were found on grass up to 14 months and at 17 months in the drop- 
pings originally placed on the soil. The propensity of larre for climbing vegetation results in rendering infective, any ostrich forder raised on land contaminated by the droppings of infected birds. Mature larvæ will remain alive in a moist atmosphere at room temperature for fifteen months. They will remain alive in water at a low temperature for 13 months. After being kept in ice at a temperature of $16^{\circ}$ to $28^{\circ} \mathrm{F}$. for 11 days and released by melting the ice, they revive. Larva will travel through soil, so no benefit can result from plowing infected ground.

The possibility that mature larvæ pass through the skin of the ostrich to reach the stomach has been tested by experiment, with negative conclusions. Sheep and fowls do not serve as hosts for the wireworm.

Mature larve outside the ostrich may be killed by a liberal application of carbolic acid or lysol in 5 or 6 per cent solution. Sulphuric and hydrochloric acids are effective.

On gaining access to the ostrich, the larva pass through the third and fourth larval stages and develop into the adult worm after the fourth molt.

Ostrich chicks may succumb to infestation by these larve in the parasitic stages, before they develop into adult worms.

Adult birds can tolerate an excessive infestation with wireworm provided they are well fed. Once infested, an ostrich will remain infective for years and constitute a source of contamination of the soil. An infected pasture or pen will remain infected for years by reason of the presence of eggs and larvæ.

Morbid anatomy. The wireworms are found in the proventriculus in the lumina of certain deep glands or lying between superficial tubular glands or in the depths of the mucosa of certain areas of the organ near the entrance of the esophagus. They are demonstrated in the fresh stomach by scraping off the mucous laver of the mucosa. They are very rarely on the surface but proceed thence upon reaching maturity. The effect of infestation becomes evident after about the 24th day after infection, and subsequently. The laver of mucus becomes detached in parts and hemorrhagic infiltration of the mucosa appears. When the worms are numerous and mature, the mucosa appears swollen and is covered by a copious amount of mucus. This condition designated "vrotmag" (rotten stomach) by the Boers, causes anemia and emaciation of the birds.

Diagnosis. The presence of worms may be determined by worm cultures, that is by placing fresh droppings in a wineglass which is 
covered to prevent evaporation. If the bird is infected, larvæ recognizable with the naked eye, will after a time be seen on the glass. The number present furnishes a rough indication of the extent of the infection.

Prevention. It is possible to raise ostrich chicks free from infection when suitable precautions are carried out. Small runs 50 by 150 feet are quite large enough. If clean ground over which ostriches have not run is not available, it is possible to render an area safe by remoring the soil to a depth of three inches. The runs should be so located that there is no contact with infected birds, and so that there is no possibility of infection through drainage into them. The runs should be stocked with incubator chicks, or chicks removed from the nest immediately after hatching. The feed will be of the usual character, derived from land that has not been contaminated by infected birds. If hens are kept with the chicks, all feces of the adult birds should be picked up daily. These precautions will permit raising ostrich chicks to such an age that wireworm infestation is no longer dangerous to the birds, and has the additional advantage of preventing sclerostome and tapeworm infestation.

Treatment. No treatment has been discovered which can be depended upon to expel all the worms from an infected bird. The location of the worms in the mucosa and glands is such as to discourage belief that a successful treatment will be found. A number of substances when tested in vitro are undoubtedly effective in killing the parasites. A number of methods of treatment have been tested by Theiler and Robertson, who judged the effect by observing the influence of the treatment on the numbers of eggs in the feces both by microscopical observation and by worm cultures.

Carbolic acid in a dose of 300 c.c. of a 2.5 per cent solution caused the eggs to become rare for 14 days after which they became as numerous as they were before treatment. Several trials with this agent led to the conclusion that the dose employed, which is equivalent to 7.5 grams of pure carbolic acid, checks the laying of eggs for a while without killing all the worms.

A treatment was tried which consists of administering 300 c.c. of paraffin (kerosene), followed after 24 hours by 1 ounce each of slaked lime and of sal-ammoniac. It has been claimed that the paraffin dissolves the mucus-like layer over the worm infested area, and that the ammonia resulting from the reaction between the drugs administered later kills the worms. However, the trial failed to demonstrate any effect upon the worms. 
Dosing with thymol in quantities as large as 12 grams had only a temporary effect in checking the laying of eggs for a few days.

Beta-naphthol in repeated enormous doses and followed after several days with 250 grams of magnesium sulphate merely checked the laying of eggs for a few days.

Santonin in 10 gram doses produced some influence on the number of eggs present after treatment.

Kamala in doses of 20 and of 40 grams produced no apparent effect. Flores koso in doses of 50 and of 100 grams merely produced some influence on the number of eggs.

Liquid ammonia proved ineffective and even harmful to birds receiving the treatment. Carbon bisulphide in a dose of 30 c.c. in 170 c.c. of olive oil or in a dose of 20 c.c. in a gelatin capsule, proved ineffective.

Apart from the fact that the worms are well protected in their location in the mucosa, certain anatomical considerations prevent drugs from acting unless the fluid fills the whole stomach and raises the fluid to the level necessary to bring it in contact with the areas infested with worms. This is almost impossible to accomplish since the intestinal opening is so low as to drain out fluid.

TAPEWORM OF THE OSTRICH

The tapeworm of the ostrich is known as Tania struthionis, and is chiefly a parasite of the young. Chicks may become infested when only three weeks old and from that age until two years, tapeworms constitute a very common parasite. The worms reach two or three feet in length and may almost plug the intestine. Robertson reports having observed in one ostrich nine months old, over two hundred tapeworms with a weight of nearly three pounds. The aggregate harm done by these in abstracting nutriment and in injuring the mucosa at the point of attachment, must have been considerable. On the other hand, the occurrence of tapeworms in the adult ostrich, is rarely observed. The intermediate host of the ostrich tapeworm is not known.

Symptoms. Under good conditions an ostrich may tolerate a heavy infestation without showing symptoms. If food becomes searee, climate becomes unfavorable, or the chick becomes ill from some cause, the bird exhibits evidence of the presence of the worms. There is general ill health, and the mucosæ of eyes and month are pale. The presence of ripe segments of tapeworm in the droppings is 
the one certain symptom. The segments are always found on the outside of the dung pellets.

Treatment. Robertson recommends the use of petrol or motor car spirit, Pratt's green label (gasoline). It is advisable to fast the chicks a little before dosing, but if weak and thin, they should not be starved before the first dose. The dosage for various ages as recommended by Robertson, is

1 and 2 months old birds receive $1 / 2$ oz.

3 months old birds receive $1 \mathrm{oz}$.

4 months old birds receive $2 \mathrm{oz}$.

5 months old birds receive $3 \mathrm{oz}$.

6 months old birds receive $4 \mathrm{oz}$.

7 to 11 months old birds receive $5 \mathrm{oz}$.

12 months old birds receive $6 \mathrm{oz}$.

Full grown birds receive $8 \mathrm{oz}$.

The petrol is given pure and not mixed with water. It volatilizes at a temperature below that of the body of the bird, and consequently permeates the whole intestinal tract in a short time.

Care must be taken in drenching, for even a little fluid gaining access to the trachea may be followed by serious or even fatal consequences. In drenching birds, the entrance to the windpipe should be covered with the fingers of one hand while the bottle of petrol is passed over them. The head of the bird should be held up for a few minutes after drenching to prevent the possibility of regurgitation.

Hutcheon has employed liquid extract of male fern in a dose varring from a teaspoonful for a young ostrich three or four months old, to a tablespoonful for a full grown bird. He has also used turpentine in a dose varying from a dessertspoonful to two tablespoonfuls. It is more effective when combined with a purgative such as linseed or castor oil.

FILARIA OF THE OSTRICH

A gigantic nematode of the ostrich, designated Filaria spicularia occurs in the subperitoneal connective tissue and also in the sublumbar region. The parasites vary in length from a quarter of a meter to a meter and are coiled together in such a complex manner that it is difficult to secure a whole specimen. The presence of the parasite appears to have no effect upon the health of the birds. 


\section{LEUCOCYTOZOÖN INFECTION IN OSTRICII CIICKS}

Walker has observed a lencocytozoön infection in ostrich ehicks varying in age from 4 weeks to 7 months.

Symptoms. There is a disinclination to feed, loss of condition, stunted growth and paleness of the buceal nuncous membrane. The skin of the body and around the eye is bluish colored. When the chicks are driven, the affected ones are unable to keep up with the rest of the flock. Sickness is noted several days before death.

Morbid anatomy. Microscopic examination of the organs shows no constant lesions. Examination of the blood frequently reveals the presence of a leucocytozoön. The observations lead Walker to the conclusion that it will be necessary to ascertain whether the leneneytozoön enters into the etiology of diseases of ostrich chicks.

Etiology. The organism is designated Leucocytozoön struthionis. It oceurs in young chicks but has not been found in adult ostriches. In dried blood smears fixed with methyl aleohol and stained with Giemsa, two main types of parasites are observed which apparently correspond to male and female gametocytes.

The female gametocyte occurs most frequently. The shape is more or less rounded but may be irregular, due to distortion brought about in preparing the smears. The organism varies from 11 to 15 microns in length and from 9 to 13 microns in width. The protoplasm stains deeper than does that of the male gametocyte and scattered through the protoplasm are a number of metachromatic granules. These appear more distinctly in some of the parasites than in others. A number of small clear spaces occur throughout the protoplasm. The nucleus is represented by an aggregation of small chromatin granules generally at the center or near the edge. In most instances a large ehromatin granule stands out distinetly. It is situated in the mass of granules, or at the side of them, or at some distance from them. The nucleus of the host cell is always altered in shape. In most cases it is elongated and located at the margin of the parasite.

The male gametocyte is usually more or less rounded and is smaller than the female. The protoplasm stains less densely than that of the female. The chromatin granules of the nuclens are generally seattered throughout the cell. Sometimes they are larger and widely separated and are observed very distinctly. The nuclens of the host cell is irregular in shape and is found at the edge of the parasite. It is less elongated and smaller than in the case of the cell invaded by the female. 
No spindle shaped formation of the host cell has been observed in smears from ostriches.

\section{ACORN POISONING IN THE OSTRICH}

Robertson has observed a number of cases in which acorns have killed ostriches. These when consumed in moderation constitute a safe and excellent feed. However, when birds have access to a bountiful supply, they will gorge themselves with fatal result. The injury is caused by the astringent action of the tannic acid.

Symptoms. The birds lie down persistently, seem constipated, strain and appear giddy. They stagger on tiptoe balancing themselves with their wings. The feces consist of nearly cylindrical pellets of very hard material. The urine closely resembles green paint. Some birds twist their necks as if in pain.

Morbid anatomy. Lungs, heart, liver and spleen appear normal. The stomach contains undigested acorns. The intestines show evidence of constipation with subsequent congestion. The duodenum is swollen, thickened and congested with blood. The mucosa shows acute inflammation and is covered with a dirty, grayish white deposit or slime. On scraping this off the mucosa is seen to be very red. The colon is packed with pellets of hard dung which are black in color and covered with a gray slime with traces of blood. The mucosa of the colon is also highly reddened. The ceca are packed with masses of hard dung.

Treatment. The affected bird is given 25 ounces of raw linseed oil to which is added 4 drams of powdered Barbadoes aloes which previously has been dissolved in a little warm water. Twelve hours later each bird is given one ounce of bicarbonate of soda dissolved in water. Birds that refuse to eat may be drenched with gruel, raw eggs and brandy several times a day.

\section{REFERENCES}

1. Archibald. Aspergillosis in the Sudan ostrich. J. Comp. Path. and Therap., Vol. 26, 1913, p. 171.

2. Jowett. Pulmonary mycosis in the ostrich. J. Comp. Path. and Therap., Vol. 26, 1913, p. 253.

3. Marx. Ueber ein Infectiöse Krankheit der Strausse. Centralbl. $f$. Balteriol. (Etc.), 1 Abt. Orig., Bd. 27, 1900, S. 822.

4. Neumann. La filaire de l'autruche (Filaria spicularia). Rev. Vét., T. 66,1909, p. 544 .

5. Robertson. Paralysis in the ostrich. J. Comp. Path. and Ther., Vol. 23, 1910, p. 182. 
6. Robertson. Notes on ostrich parasites. Agr. J. Cape of Good Hope, Vol. 33, 1908, p. 583.

7. Theiler. Anthrax in the ostrich. Agr. J. Union of So. Africa, Vol. 4, 1912, p. 370 .

8. Theiler and Robertson. Investigations into the life history of the wire-worm in ostriches. Union of So. Africa, Dep. Agr., Ann. Rpts. Dir. Vet. Res., 3-4, 1915, p. 215.

9. Walker. Aspergillosis in the ostrich chick. Union of So. Africa, Dep. Agr., Ann. Rpts. Dir. Vet. Res., 3-4, 1915, p. 535. 


\section{CHAPTER XIX \\ INFECTIOUS DISEASES OF GEESE AND DUCKS}

GOOSE SEPTICEMIA

History. Curtice in cooperation with Smith described several outbreaks of hemorrhagic septicemia among geese in Rhode Island. The disease occurred in one establishment among geese kept in lots of 300 each in fattening pens. The birds were assembled from varicus farms in numerous small lots, any one of which might have contained infected birds. Subsequent handling of the birds in the fattening pens involved separating them according to endition, which practice brought about extensive intermingling of birds.

Etiology. The etiological agent is a member of the hemorrhagic septicemia group which has become well adapted to waterfowl, geese in particular.

Pathogenicity. Geese are susceptible to infection by feeding cultures while ducks and hens are not. Geese, ducks, rabbits, mice and pigeons are susceptible to subcutaneous injection and hens are not. After exposure by feeding or by subcutaneous inoculation, death occurs in less than thirty-six hours. Feeding culture to a goose may cause death in less than seventeen hours.

Symptoms. It is characteristic of the disease that birds are found dead at feeding time, mornings or evenings, without having shown sickness. Symptoms occasionally are observed during the last hour or so before death and largely concern the death struggles. The gait becomes uncertain, the head is burrowed in the dirt and is twisted.

Morbid anatomy. There is considerable mueus in the throat and mouth, and very tenacious mucus in the nasal cavity. The veins of the head seem congested as if the bird had died of asphyxia. 'The mucosa of the intestine is studded with ecchymoses or larger hemorrhagic areas. In about half of the cases, the liver is studded with necrotic foci. Epicarditis and pericarditis are present occasionally as also is involvement of the lung.

Treatment. Medicinal treatment is unavailing.

Prophylaxis. Preventive measures will include precautions 242 
such as restricting the number of birds in enntact with one another and other similar precautions aimed at restricting the spread of infection.

\section{EXUDATIVE SEPTICEMTA OF GEESE}

Synonyms. Ansteckende Luftsackentzündung der Günse, GänseInfluenza.

Characterization. Exudative septicemia is an infectious septicemic disease of geese distinguished by the formation of fibrinous exudate upon the pericardium, liver and intestines and caused by Bacillus ansemım exudative.

History. The disease has been observed by Frosch and Birnbaum, Löftler and also by Riemer. Bugge studied a similar disease and while he does not give details concerning the organism present, it is possible that he encountered the same infection.

Geographic distribution. The disease seems to have been recognized unquestionably only in Germany. The occurrence of the disease among geese imported into Germany from Russia would indicate that it exists in the latter country also. II'Fadyean has described a septicemia observed in geese in England which resembles the disease in question in several particulars.

Symptoms. The existence of the disease is first indicated by loss of appetite. The breathing may be accelerated, with the beak open and accompanied by a snoring sound. Coughing and choking may occur. The bird separates from the flock, squats frequently and rises with difficulty. Diarrhea occurs within 24 hours of death. The bird becomes progressively weaker and dies in from two to five days.

Morbid anatomy. The most striking lesion is the presence of a fibrinous exudate upon various structures in the body cavity. The surface of the liver is covered by a thin, fibrinous, yellowish white layer which is easily detached. The exudate may extend between loops of the intestines, the air sacs, the pericardium and the epicardium. Fluid exudate may also be present. The pericardial sace contains serous, cloudy fluid. The bronchi in some cases are plugged with yellowish, fibrinous, purulent material. The liver is usually slightly enlarged and may contain small spots. The spleen and kidneys are also enlarged. The mucous membrane of the intestine is swollen, reddened and emtains small hemorrhages.

Etiology. The infection is caused by Bucillus septicemiu unserum exudative described by Riemer. This is a small slim rod varying in length from .5 to 1.5 microns and .5 to 1 micron broad. 
The cells frequently lie in pairs, so that they appear like diplococci. The organism is always found in great numbers in the heart blood, the pericardial fluid and in the fibrinous exudate. It is stained readily by the common anilin dyes, and best by carbol fuchsin. The organism is Gram negative, non-motile and does not form spores. Indol production is not observed except perhaps in traces in old cultures.

Frosch and Birnbaum succeded in growing the organism at first only on medium containing hemoglobin. The first generation out of the goose grew on plain agar and bouillon when plenty of blood was carried over to the medium in making the inoculation. Pigeon blood agar induced growth but after many transfers failed. A medium rich in hemoglobin made as follows was more successful. Defibrinated horse blood is mixed with equal parts of distilled water to cause the liberation of the hemoglobin. About 1 c.c. of this solution is added to each tube of melted, cooled agar. The agar is then shaken and is subjected to fractional sterilization at $70^{\circ} \mathrm{C}$. for three days. On hemoglobin agar so prepared, the organisms form a luxuriant grayish white growth. In smears from such cultures the rods appear somewhat larger than in smears from the animal body and very frequently long thread-like forms are observed.

On common agar of slightly alkaline reaction it forms in 24 to 36 hours a luxuriant grayish white, transparent, slightly opalescent layer, which in older cultures becomes brownish yellow in color. The condensation water contains considerable sediment. The optimum growth temperature is $37^{\circ}$ to $38^{\circ} \mathrm{C}$. No growth occurs on acid agar.

The period of life of a culture is limited. By holding at room temperature, transfers succeeded at 14 days, but many transfers fail sooner. The cultures in the incubator nearly all die within 8 days.

A slight uniform clouding occurs in bouillon, which after some days contains a limited amount of sediment which on twisting, forms a spiral column. In some cultures a pellicle is formed of threadlike growth.

In gelatin stab cultures held at $21^{\circ} \mathrm{C}$, a slight non-characteristic growth is observed in the vicinity of the line of inoculation, and on the surface. After some time a funnel-shaped area of liquefaction forms slowly, and complete liquefaction eventually occurs. Growth on gelatin plates is accompanied by liquefaction and offers no characteristic features. 
Glucose and lactose bouillon are uniformly clouded but gas formation does not occur. In lactose there is no change in reaction, but in glucose there is a slight reduction of alkalinity.

A luxuriant growth occurs in the form of a whitish yellow layer on Löffler's serum. On potato a thin shiny, yellowish white layer is observed at $2 t$ to 48 hours, which later becomes brownish in color. Growth does not occur on Drigalski-Conradi nor Endo plates. Milk is not coagulated. Riemer and Löfler both regard the organism as belonging to the influenza group.

Pathogenicity. The organism is markedly pathogenic for geese, which are infected most certainly by intramuseular injection. The musculature in the vicinity of the point of inoculation is colored grayish white over a large area. An attempt by Frosch and Birnbaum to infect a grown goose by feeding with organs of a goose dead of the disease, failed, as did a similar experiment by Riemer in which bouillon culture was used. In neither case were goslings available.

The latter author observed that either intramuscular or subcutaneous injection caused sickness followed by death in from 36 to 72 hours. In artificially infected birds the fibrinous exudate on the liver may not be found, due to the unnatural rapidity of the course of the disease.

Rabbits, gray and mixed rats, mice, white mice, hens, and pigeons are refractory to inoculation. Guinea pigs have succumbed to an intraperitoneal inoculation of a suspension of the growth from one agar culture. They withstand smaller amounts, such as 1 to 3 loops.

Riemer succeeded in infecting an 8 weeks old duck by intramuscular injection of a suspension from an agar culture. Of three grown ducks, only one became infected by intramuscular injection.

M'Fadyean in England examined a few geese dead from a septicemic infection. Smears from the blood contained great numbers of organisms which were cylindrical rather than ovoid in outline. Beyond a slight inflammation of the intestine, no lesions were observed. Agar tubes seeded from the heart blood failed to show growth, and cultures kept under anaerobic conditions likewise failed. The organism was Gram negative, and was not pathogenic to the mouse, the rabbit, the hen, the gninea pig, the pigeon or to one duck. Transmission to a goose by feeding failed, but succeeded when subcutaneous inoculation was employed. The writer concluded that the disease was not fowl cholera. None of the observations made deny the possibility that the infection was exudative septicemia. 
Prevention. Individual isolation of birds is the most effective means of preventing the spread of the infection. General disinfection is indicated.

\section{OSTEO ARTHRITIS IN YOUNG GEESE AND DUCKS}

Synonyms. Ostéo arthrite aigue des jeunes oies, French; osteoarthritis bei jungen Gänsen und Enten, German.

Characterization. The affection consists principally of a serous or sero-fibrinous arthritis, hemorrhagic inflammation of the bone marrow and intestinal catarrh.

Geographical distribution. Outbreaks have been reported in France, Germany and in the latter country among birds that had recently been imported from Russia. It is reported as generally distributed in Germany.

History. The disease was first described by Lucet in France in 1892, by Freese in Germany in 1903, and by Hasenkamp and Sachweh in the same country in 1914.

Etiology. Pus from all joints examined bacteriologically yields pure cultures of Staphylococcus pyogenes aureus which, however, forms but little pigment. The organism is recognized microscopically in pus in great numbers and a few are observed in smears from heart blood.

Pathogenicity. Only young geese and ducks are susceptible, of which 90 per cent become affected. Old geese and ducks, hens and pigeons are immune. Hasenkamp and Sachweh report one instance of finding a dead hen in an establishment where ducks were infected, but made no bacteriological examination.

The hen, pigeon and rabbit injected subcutaneously with blood of an infected bird, remain healthy. The hen and pigeon likewise are not affected by subcutaneoris injection of pus from the joint of an affected bird. Hasenkamp and Sachweh have infected hens and pigeons by intravenous inoculation.

The disease is transmitted to young ducks by intravenous inoculation with culture of $S$. pyogenes aureus. Subcutaneous inoculation or ingestion have failed to induce infection.

Mortality. Löfler observed an outbreak of the disease among 346 geese, of which 43 were old, and 313 young ones. Of the 346 geese, 191 became sick. Of these latter 5 were old and 186 were young. One old one and 159 young died. The mortality among the young ones was 85.5 while that of the old ones was 20 per cent. 
Symptoms. In general the disease is manifested in two forms, either as a pure acute type with rapidly fatal result, or as a chronic form terminating in death or recovery after a long time.

In the acute type as described by Freese, young geese and ducks appear apathetic and eat less. When driven it is seen that they are very lame in one or the other leg. The joints of the leg's are swollen, fluctuating, hot and very painful. Usually only a few joints are involved, among them most frequently the hock and single toe joints. The wing joints may also be involved and of these frequently the elbow is involved. In this ease, the affected wing droops. Besides symptoms of arthritis one observes severe diarrhea and often a slight mucous catarrh of the conjunctivæ. Birds showing the acute symptoms die in from two to four days.

In the chronic form of the disease the arthritis is the most prominent feature of the clinical picture while the general symptoms are less marked. There is a slight diarrhea during the first few days and appetite does not entirely disappear. The birds do not appear quite apathetic but frequently make efforts to walk. They become greatly emaciated. These symptoms may last for various lengths of time, on the average, 14 days. Then, either death occurs or the birds improve with gradual disappearance of symptoms of acute arthritis. Nevertheless in many cases a painless swelling of certain joints remains for weeks. In this case the birds walk stifty for a long time. They remain halted in development so that they are not suitable for fattening. In isolated cases during the chronic course, an acute relapse may occur followed by death.

Lucet describes two types of the disease. One, designated as the peracute type, is rapidly fatal. In this, the osseous lesions are slightly pronounced or sometimes inappreciable. The other trpe, the acute, is accompanied by epiphyseal osteitis and arthritis. The duration of the disease is much longer and is terminated by death after a variable time or by recovery.

In the acute type as observed by Lucet there is depression, the head is bowed, the feathers are ruffled, dirty and dull. The muensix are pale, diarrhea oceurs, there is no appetite and the rectal temperature is markedly elevated. The infected subjects lag behind the others, walk with difficulty, limp and assume a cronching position frequently. All these symptoms rapidly become aggravated. The upright position becomes impossible, the members are incapable of movement and the collapse is extreme. The head rests on the ground by the beak. Death occurs in one, two or three hours. 
The acute form is much more common and because of its slower development is much better defined. First there is depression, ruftling of the feathers, diarrhea, elevation of the rectal temperature and loss of appetite all of which are common to serious diseases. This period of development more or less accentuated has a somewhat irregular duration. Sometimes it precedes the local symptoms by several days, at other times it is hardly appreciable. Walking becomes difficult and painful. There is lameness in one or both legs. The patient lags behind its fellows, sits on the ground and assumes the upright position with difficulty. At this stage the important joints, especially the tibio metatarsal joints, singly or together are the site of precise pathological manifestations. They are enlarged, doughy, very painful and execute very limited movements. Sometimes they are completely immobilized. The temperature reaches $36^{\circ}$ to $38^{\circ} \mathrm{C}$. when under normal conditions it would be $30^{\circ}$ or $34^{\circ} \mathrm{C}$.

Incapable of moving, the geese remain in the sternal position for entire days with the feet underneath them or extended behind or even sideways. The wings droop, the bird is depressed, holds the beak down and eats with pain. Sometimes one which is less ill attempts to flee when approached. With neck stretched out, with beak opened, and whistling it flaps the wings and drags itself along for several steps before stopping exhausted.

In such a condition, two terminations are possible. There may be increase of depression, diarrhea and pallor of the mucous membranes. The cutaneous parasites become more numerous, the emaciation and cachexia increase and death follows after one, two or three weeks. On the other hand, the general symptoms diminish in intensity, the appetite returns, the movements become easier and recovery occurs after a variable time.

Complete recovery is rare. The affected joints never return to their normal size nor their former mobility. Ankyloses, thickenings and irregularities of the joints interfere with movement.

Morbid anatomy. The carcass is greatly emaciated. The mucosa of the beginning and end portions of the intestine is greatly swollen and diffusely reddened or slate gray in color. The abundant contents of the intestines are viscid and possess a reddish gray color. The intestinal inflammation is most marked in the pure acute type in which case, dark red petechir oceur in the mucosa. The spleen is hypertrophied and the liver is always enlarged.

The affected joints are more or less swollen and filled with either 
a pure serous fluid or a sero-fibrinous exudate. The fibrin occurs mostly as a yellowish, transparent layer on the joint cartilages and on the inner wall of the joint capsule.

The exudate is present in various amounts. The synovial membrane of the joint capsule is swollen and reddened in the acute cases.

The bone marrow is very shiny, dark red in color and the consistency is strikingly soft, often fluid.

In the chronic form the joint cartilage is involved in spots and is easily lifted from the tissues beneath. Under these spots, the bone has a porous, rough consistency. In the epiphyses of the bone are noted cavities of various sizes which are filled with dry, crumbly, grayish yellow masses. These when rubbed between the fingers feel granular and are regarded as bone pus. Freese observes that pus in birds has this consistency instead of the creamy-like character of that occurring in mammals. In other epiphyses there are holes in the joint cartilage which communicate directly from the joint to the cavities in the bone described above. In such cases the joints contain more or less of the bone pus. The bone of the altered epiphyses is covered on the outer surface with a cartilage-like deposit, which is easily overlooked.

Prevention. Prophylactic measures should include separation of the sound birds from infected ones, disinfection of the quarters, proper disposal of the dead and of the manure.

Treatment. The advisability of local treatment with counter irritants and disinfection might be considered.

SEPTICEMIA OF DUCKS

Lisi has described a fatal septicemia of ducks in which all birds exposed to the infection succumbed.

Etiology. The causative organism is present in the rarious exudates, organs and in small numbers in the blood. It is somewhat larger than that of fowl cholera. The organism grows on gelatin, causing slow liquefaction and forms a brown colored layer on potato.

Pathogenicity. Inoculation of ducks with culture causes death in ten or twelve hours. Rabbits and guinea pigs suecumb in three days. The fowl is refractory. The white rat is somewhat susceptible and an area of caseons infiltration forms at the point of inoculattion.

Symptoms. The advent of symptoms is abrupt, the eyes are closed and lachrrmating. The bird appears depressed and remains 
in one position a long time without moving. Death occurs in from one to four hours.

Morbid anatomy. The eyelids are closed with a purulent secretion, the cornea is covered with white spots, the aqueous humor sometimes is opaque. The pericardium contains a gelatinous exudate and the heart muscle is pale. The liver is softened, the intestines and mesentery are congested. A fibrinous exudate occurs on the peritoneum of some birds.

\section{DUCK CHOLERA}

Cornil and Toupet described a disease of ducks which resembles fowl cholera very closely. The authors differentiate it from that disease on the basis of the non-susceptibility of fowls and pigeons to cultures of the organism isolated from the ducks.

\section{SEPTICEMIC INFECTION OF WATEREOWI,}

Willach observed a cholera-like infection among waterfowl. The outbreak involved Pekin ducks, swans and geese.

Symptoms. The affected birds show dullness, apathy, inappetence, marked thirst, difficulty in swallowing and hoarseness. They die suddenly without spasms.

Morbid anatomy. Autopsy reveals lesions of fowl cholera.

Etiology. Examination of the blood reveals an organism resembling that of fowl cholera. However, it differs from it in several particulars.

Pathogenicity. Hens, ducks, pigeons and mice succumb to the infection.

\section{REFERENCES}

1. Bugge. Ansteckende Luftsackentzündung der Gänse. Zeitschr. $f$. Infektionskr. d. Haustiere, Bd. 3, 1907, p. 470.

2. Curtice. Goose septicemia. Rhode Island Agr. Exp. Sta. Bull. 86, 1902.

3. Cornil et Toupet. Sur eine nouvelle maladie bacterienne du canard (choléra des canards). Compt. rend. Soc. de biol., T. 106, 1888, p. 1747.

4. Freese. Ueber eine durch den Staphylococcus pyogenes aureus hervorgerufene Osteo-Arthritis bei jungen Gänsen und Enten. Deutsche tierärztl. Wchnschr., Bd. 15, 1907, S. 322.

5. Frosch u. Birnbaum. Ueber eine durch den Bacillus septicæmiæ anserum exulative (Riemer) bedingte Gänsesenche, Zugleich ein Beitrag zur Frage dere Pseudoinfluenzabacillen. Centralbl. f. Bakteriol. (Etc.), 1 Abt. Orig., Bd. 52, 1909, S. 433. 
6. Hasenkamp und Sachweh. Staphylokokken-Erkrankungen beim Geflugel. Tierärztliche Rundschau, Bd. 20, 1914, S. 85.

7. Löfller. Ueber eine in Jahre 1904 in Klein-Kiesow bei Greifsweld beobachtete Gänseseuche. Arch. f. Tierheilk., Suppl. Bd. 36, 1910, S. 289.

8. Lisi. Infezzione setticoemia in una branco di anatrini. Il moderno zooiatio, 1896, p. 415.

9. Lucet. De l'osteo-arthrite aigue infectieuse des jeunes oies. Ann. de l'Inst. Pasteur, T. 6, 1892, p. 841.

10. M'Fadyean. A remarkable outbreak of goose septicemia. J. Comp. Path. and Therap., Vol. 15, 1902, p. 162.

11. Riemer. Kurze Mitteilung über eine bei Gänsen beobachtete exsudative Septikämie und deren Errger. Centralbl. f. Bakteriol. (Etc.), 1 Abt. Orig., Bd. 37, 1905, S. 641.

12. Willach. Eine Cholera unter dem Wassergeflügel in Schwetzingen. Deutsche tierärztl. Wchnschr., 1895, No. 51. Abstracted in Centralbl. f. Bakteriol. (Etc.), 1 Abt. Orig., Bd. 20, 1896, S. 187. 


\section{CHAPTER XX}

\section{INFECTIOUS DISEASES OF CANARY BIRDS}

Infectious diseases of canary birds cause heavy losses in breeding establishmen:s where hundreds of birds are kept in close contact with one another. These conditions, and the occasional introduction of breeding stock furnish favorable circumstances for the spread of disease, and severe losses occur. The literature contains references to a number of infections chiefly observed in Germany where canary breeding attains considerable importance. Careful comparison of the descriptions of canary bird infections indicates that most of those described fall into one of two groups. One, designated infectious necrosis is caused by a member of the hemorrhagic septicemia group, and another is caused by $B$. paratyphosus $B$.

\section{INFECTIOUS NECROSIS OF CANARIES}

Synonyms. Bird fever, canary fever, septic fever and bird plague.

Characterization. The disease is an infection ehiefly distinguished by the presence of necrotic lesions in the spleen and liver.

History. The disease has been described by a number of writers including Rieck, Wasielewski and Hoffman, Pfaff, Zwick, Miessner and Schern, Zeiss, and Binder. The last two writers independently, have thoroughly reviewed the literature of canary bird diseases. Doubtless the diseases observed by Kinyoun and by Ziirn are the same, and in the opinion of Zeiss the one observed by Kern is identical. On the other hand, Binder withholds decision as to whether or not Kern's disease is really the one in question. Most of the writers have refrained from giving the disease a name, but MFiessner and Schern suggested the name infectious necrosis.

Etiology. The organism causing the disease is a non-motile rod measuring from 1.5 to 2.5 microns long and .5 to 1 . micron broad. Occasionally larger forms are observed. The organism is Gram negative and shows bipolar staining. Surface colonies on agar are grayish in color, appear distinctly granular and are about the size of a pinhead. No growth occurs on plain potato, but when this medium has been rendered alkaline a slight brownish colored layer 
forms. Boullon beemes slightly elouded and abundant secliment accumulates. Milk is not coagulated and no gas is formed in sugar agar. Gelatin is not liquefied. Blue colonies form on Drigalski plates. A trace of indol was observed by one writer.

Several writers who have worked with the organism regard it as a member of the hemorrhagic septicemia group. Miessner and Schern name the organism Bacillus canariensis necrophorus.

Pathogenicity. The organism induces disease in canaries when introduced subcutaneously and per os. The disease must progress for at least three days in inoculated birds before the characteristic necrotic lesions develop. Mice, sparrows, finches, guinea pigs and rabbits are also susceptible. Pigeons vary in susceptibility while hens are resistant.

Symptoms. There is nothing characteristic about the behavior of birds harboring this infection. The birds lose appetite, become less lively and finally squat in a corner of the cage. Death occurs after a sickness of from 24 to 36 hours.

Morbid anatomy. The characteristic feature observed at autopsy is the presence of metastatic foci in the spleen, liver and occasionally in other organs.

The liver is enlarged and contains numerous yellow foci the size of a pinhead and larger as seen by the unaided eye. The use of a hand lens reveals smaller punctiform foci. These areas present a great variety of shapes, and do not separate readily from the surrounding tissue. They are sometimes leathery in texture, sometimes crumbly in structure. The larger ones when cut, are seen to consist of a homogeneous central mass surrounded by an opaque, gray colored zone.

The spleen is swollen into a cylindrical form and contains similar nodules. These distend the capsule and cause the surface of the organ to have an irregular, undulating appearance. The spleen contains so many nodules and is so fragile in consequence, that it breaks apart when grasped with tweezers. The mucous menbrane of the pharynx occasionally contains yellowish nodules which may be detached with the tweezers. The walls of the pleural cavities occasionally are covered with yellowish exudate, and the lungs may be involved. Intestinal inflammation is sometimes observed.

The necrotic lesions closely resemble those of avian tubereulosis, but tubercle bacilli are not demonstrable.

Stained sections of the nodules in the organs reveal the fact that the smaller ones consist of great clumps of bacteria which have 
multiplied at the places in question. The larger ones consist of clumps of bacteria surrounded by necrotic cells. The largest nodules consist of a number of smaller ones united together.

The presence of the organism in the heart blood is not always demonstrated microseopically or by culture.

Diagnosis. The disease can be recognized only by the characteristic lesions observed at autopsy.

Treatment. No medical treatment is available.

Prevention. General sanitation and isolation as recommended in paratyphoid B infection is suggested.

\section{INFECTION IN CANARIES CAUSED BY B. PARATYPHOSUS B}

Synonyms. Septic fever in cage birds.

Characterization. The disease is characterized by enlargement of the spleen with enteritis and is caused by $B$. paratyphosus $B$.

History. The disease was described by Joest in 1906 and has been encountered later by Gilruth, Pfeiler, Adam and Medler, Manninger and by Binder. The latter writer and also Zeiss have summarized the work of earlier students of canary bird infections.

Etiology. The organism isolated from the birds is a short plump rod 2 to 4 microns long and $1 / 2$ to 1 micron broad with rounded ends. It is actively motile and Gram negative. Flagella may be demonstrated by Löfler's method, but with all care, it is possible to demonstrate one flagellum only on the end of the rods. The organism in fresh smears from heart blood or organs often shows bipolar staining.

Growth on agar, gelatin, bouillon, blood serum and potato is the same as that of cultures of $B$. paratyphosus $B$ and $B$. supiestifer. Neither can differences be discovered between the growth of these cultures and the canary strains on Drigalski and Conradi or on Endo's fuchsin agar plates.

In all strains, milk becomes brightened and yellowish colored after 8 days. After 14 to 20 days it becomes markedly yellnw and transparent. After standing in the incubator for a longer time it becomes thickened, viscid and yellowish brown in color. Acid and gas are produced in dextrose, arabinose, rhamnose and zylose but not in lactose, saccharose, and raffinose. Indol is not produced.

The organism agglutinates to $B$. paratyphosus $B$ serum and paratyphoid B cultures of human origin agglutinates to serum prepared from the canary strain. 
Pathogenicity. The organism is highly virulent for canary birds, mice, guinea pigs and rabbits.

Symptoms. In the beginning of the disease the bird is not so lively as usual and sits on the perches with rufted feathers. Later the head is turned backward between the wings, which latter droop. Occasionally the bird hops about briskly and eats. As the disease progresses the bird becomes more depressed, appetite ceases entirely, the droppings become thin, the eyes are kept half closed and respiration becomes quickened to 150 per minute. Occasionally the bird peeps hoarsely. MLovements become uncertain, the bird falls down, loses consciousness and dies in a spasm.

Morbid anatomy. Rigor mortis is pronounced. The legs are most often extended from the body. There is more or less marked inflammation of the intestines. The spleen always shows hyperemic enlargement, twice to five times its normal size. There is hyperemia of the liver and kidneys. Smears from heart blood and organs, contain great numbers of short, thick rods in pure culture.

Prophylaxis. The first measure should consist of separating the sound from the diseased birds. Further the healthy ones should be isolated in separate cages so far as possible. The droppings should be removed daily and be burned. Feed and water containers should be removed from the eages daily and be disinfected.

Treatment. Sick birds may be supplied with drinking water containing 3 to 5 grams of sulphate of iron per liter. It is best to separate them so that there will be one or at the most two to a cage.

DISEASE OBSERVED BY FREESE

Freese has described a septicemic disease of canary birds substantially as follows:

Symptoms. The bird at first ruffles the feathers occasionally and is not so lively as usual. On the next day it sits quietly on the perch, peeps now and then and shows a certain degree of dyspnea. There is smewhat more thirst than usual but the appetite is not changed. In addition, there may be slight diarrhea. It is characteristic that the sick birds at frequent intervals hop around in the cage like healthy birds. The symptoms become more marked and the birds die in a day or so. Some hours before death they sit with ruffled feathers, on the perch or in the corner of the cage. The eyes are half closed and the head is turned backwards and held in the feathers. At this time there is a great increase in the frequency 
of respiration. It is noteworthy that the birds often continue to eat, up to a few hours before death. The duration of the disease is from two to three days.

Morbid anatomy. The blood is coagulated and dark red in color. The mucosa of the duodenum is swollen and diffusely reddened. The liver is either very much congested or fragile and yellowish in color. In most cases the spleen is without microscopic lesions but occasionally there is a hyperemic swelling of that organ.

Etiology. In smears from the heart blood stained with the common anilin dyes there are seen small rods 0.5 micron to 1.5 microns long which stain uniformly. In some cases an organism can only be found after long search. Bacteria are found very sparingly in smears from liver, spleen, and kidneys of fresh carcasses. In carcasses which have lain for several hours the bacteria are found in larger numbers. That these are not post-mortem invaders is shown by the fact that pure cultures of the organism in question may be obtained from such material. The organism is Gram positive in culture as well as in smears from tissues and is non-motile. It grows on all the common culture media at incubator and at room temperature but somewhat more slowly under the latter condition. The organism thrives best with access to air and less well anaerobically.

After twelve hours on slanted agar at incubator temperature the organism forms sharp bordered, distinetly prominent, shiny colonies about the size of a poppy seed. These are thickest in the center and become uniformly thinner toward the border. By reflected light they are grayish white in color. By transmitted light they are bright, transparent and have a bluish shimmer. On long standing these colonies do not become larger and do not ehange in appearance. Such isolated colonies develop after a light seeding of material such as heart blood from a fresh carcass. After rich seeding of material and after transfer from a culture a more uniform layer forms, especially in the vicinity of the condensation water, which however, by close examination is seen to consist of colonies shaped like fine dew drops. After about twenty hours, growth ceases. At room temperature, growth is observed after twenty hours and stops at about thirty-six hours.

The organism grows in a similar manner on four per cent glycerine agar, one per cent glucose agar, and on blood serum. On the latter medium growth appears first after twenty-four hours at incubator temperature and continues about three days. After this 
time there has formed a very shiny, thin layer, the border of which appears finely toothed.

'The organism remains living on agar for six weeks without transferring and on blood serum for eight weeks.

On agar plates after fourteen hours at incubator temperature there are formed sharply defined, gray-white colonies about the size of a poppy seed which lie partly on the surface and partly within the medium. They do not change in appearance after a longer time.

On gelatin plates one observes with the unaided eye, after forty hours, fine gray-white points. By reflected light under the microscope with low magnification colonies are seen to have a brownish yellow color, have sharp borders and are circular or oval in form. In the center of these are a number of dark brown granules and on this account the center appears darker than the periphery. After two days a bright, transparent area forms about each colony and after three days one observes distinet liquefaction. This advances until the whole plate is liquefied after five days, reckoned from the time that the culture was planted. Similar growth is observed on one per cent glucose gelatin plates.

On slanted gelatin a distinct liquefaction occurs along the line of inoculation after forty-eight hours. On the bottom of the tube there is observed a large amount of cloudy fluid which has run down from the surface of the gelatin leaving a trough-like depression. In gelatin stab cultures after forty-eight hours punctiform colonies are observed along the line of the stab. There is a depression on the surface the size of a pinhead, which represents the begimning of liquefaction.

The liquefaction progresses and in about fourteen days the whole of the medium becomes liquefied. Then there is observed a graywhite viseid mass in the bottom of the test tube which when shaken, ascends and forms a dense cloudiness. The organism remains alive on gelatin without transfer for about six weeks.

In bouillon in the incubator after fourteen hours, the organism causes a uniform clouding and on shaking, a viscid sediment rises. The clouding continues till the third day and the sediment increases at the same time. After five days the upper part of the bouillon becomes somewhat more clear and after fourteen days it becomes entirely clear. In the bottom of the test tube at this time there is an aceumulation of brownish gray, thick sediment which on whirling the tube appears granular and stringy. No pellicile forms on the surface of the bouillon. Similar growth is observed in glycerine 
bouillon and in glucose bouillon. At room temperature growth in bouillon is somewhat more slow. The organism remains alive in bouillon for about four weeks.

After 18 hours, growth occurs on potato in the form of prominent, sharply outlined grayish-white colonies about the size of a poppy seed. In the middle of the inoculated area these coalesce into a uniform layer. After two or three days the culture frequently becomes brownish yellow in color in the thicker parts. The organism grows on potato at incubator and at room temperature equally well, and remains alive on this medium for six weeks.

In whole milk at incubator temperature after 15 hours a marked separation of the whey occurs. The casein is coagulated in large flakes. After 36 hours the coagulation process is completed. At room temperature coagulation occurs first at 32 hours. In spite of the lactic acid, the organism remains alive four weeks without transfer.

The organism forms no gas in the culture media employed and no acid, with the exception of that formed in milk. It develops no specific odor.

Indol is not detected in bouillon cultures four days old.

It is to be noted that in general the organism, in fresh cultures on the various media has the same form. In these, however, it appears shorter than in smears from tissues. Occasionally short, plump rods and very rarely, division forms occur. Among these may be observed many which are similar to a diplococcus. The organism appears somewhat smaller on blood serum than upon the other media. The organisms when grown on potato are the largest and longest.

Pathogenesis. Hens, pigeons, rabbits and guinea pigs are insusceptible to subcutaneous injection of culture or to injection of heart blood of a canary bird dead of the disease. In these birds it causes only a straw yellow focus about the size of a pea, at the point of injection.

The infection may be transmitted to canary birds by inoculation and by feeding. Sparrows and mice are also susceptible.

Freese compares the disease found by him with fowl cholera and with the diseases described by Rieck, Kern and Pfaff. He concludes that it differs from all of them. The one described by Kern shows the greatest similarity. The two practically agree in symptoms, lesions and pathogenesis, but cultural characters show some 
marked differences. No subsequent writer seems to have encountered the disease described by Freese.

\section{SEPTIC ENTERITIS OF CROSS BILLS}

Tartakowski has described a disease occurring in cross bills (Loxia curvirosa and pityopsittacus), gold finches (Carduclis elegans), green finches (Chrysonitris spinus) and rarely in canary birds.

Symptoms. The bird exhibits periods of dulluess alternating with periods of apparent complete health. There are frequent attacks of weakness, somnolence with impairment of appetite, and increased thirst. The disease causes death in from 10 to 12 days. During the last few days the bird sits on the bottom of the cage with eyes closed.

Morbid anatomy. When the disease has been of long duration the carcass shows great emaciation. There are no ecchymoses or exudates in the serous cavities. The breast muscles are always yellow as if cooked. Spleen and liver are always enlarged. Kidneys and heart muscle are yellow and clouded. Rarely there is marked hyperemia of the intestinal canal. Organs of respiration are normal. The brain is anemic.

Etiology. There is constantly found in the spleen, liver and blood a rod which measures from 2. to 2.5 microns long and varies from .6 to 1 . micron thick. The organism is actively motile and Gram negative. There is nothing distinctive about the growth on agar and gelatin. Gas formation occurs. There is no liquefaction in the latter. Milk is not coagulated. Tartakowski has designated the organism as Bacillus loxiacida.

Pathogenesis. The organism is fatal to cross bills when administered in drinking water, and when injected subeutaneously or intramuscularly. In guinea pigs a local swelling is induced at the point of inoculation together with transient fever. Intraperitoneal inoculation causes a sero-fibrinous peritonitis and death in from one to two days. Rabbits injected the same way do not always die from peritonitis.

\section{REFERENCES}

1. Adam u. Medler. Ueber Paratyphus-B-Infektionen bei Kanarienvïgeln und Untersuchungen ïher das Vorkommen ron Bakterien der TíoliTyphusgruppe im normalen Ǩanarienvogeldarm. Centralul. f. Batiteriol. (Etc.), Orig., Bd. 62, 1912, S. 569. 
2. Binder. Ueber die infektiose Nekrose der Kanarien. Wien. tierärztl. Wchnschr., Bd. 1, 1914, S. 337.

3. Gray. Disease in canaries. Vet. Rec., Vol. 22, 1910, p. 753.

4. Gilruth. Diseases of canaries. Vet. J., n. s. Vol. 17, 1910, p. 655.

5. Joest. Eine durch Bakterien der Enteritisgruppe verursachte Kanarienvogelseuche. Ber. ü. d. k. Hochschule zu Dresden, Bd. 1, 1906, S. 110.

6. Kern. Eine neue infectiöse Krankheit der Kanarienvögel. (Kanariencholera) Deutsche Ztschr. f. Tiermed., Bd. 22, 1896, S. 171.

7. Kinyoun. Vogelpest. Preliminary note. Centralbl. f. Bakteriol. (Etc.), 1 Abt. Orig., Bd. 38, 1906, S. 329.

8. Miessner u. Schern. Die infektiöse Nekrose bei den Kanarienvögeln. Arch. f. Tierheilk., Bd. 34, 1908, S. 132.

9. Pfaff. Eine infektiöse Erkrankung der Kanarienvögel. Centralbl. f. Bakteriol. (Etc.), 1 Abt. Orig., Bd. 38, 1905, S. 275.

10. Manninger. Ueber eine durch den Bacillus paratyphi B. verursachte Infektionskrankheit der Finken. Centralbl. f. Baliteriol. (Etc.), Bd. 70, 1913, S. 12.

11. Pfeiler. Ueber ein seuchenhaftes, durch Bakterien aus der Paratyphusgruppe verursachtes Kanariensterben. Berl. tierärztl. Wchnschr., Bd. 27, 1911, S. 953.

12. Rieck. Eine infectiöse Erkrankung der Kanarienvögel. Deutsche Ztschr. f. Tiermed., Bd., 15, 1889, S. 68.

13. Tartakowski. Ueber eine Infektionskrankheit der Kreuzschnäbel und anderer Zimmer-und Singvögel. Arch. d. Veterinärwesenschaften, 1898. (Russian.) Abstracted in Centralbl. f. Bahteriol. (Etc.), 1 Abt. Orig., Bd. 25, 1899, S. 89.

14. Wasieliewski u. Hoffmann. Ueber eine seuchenhafte Erkrankung bei Singvögeln. Arch. f. Hyg., Bd. 47, 1903, S. 44.

15. Zeiss. Beitrag zur Frage der Erreger von Kanarienvogelseuchen. Arch. f. Hyg., Bd. 82, 1914, S. 1.

16. Zwick. Untersuchungen über eine Kanarienvogelseuche. Ztschr. $f$. Infehtionskr. d. Haustiere., Bd. 4, 1908, S. 33. 


\section{CHAPTER XXI}

\section{TUMORS IN FOWLS}

Tumors in fowls hardly constitute a serious economic problem. However, in view of their great similarity in structure to those of man, they have received considerable attention.

\section{FREQUENCY OF OCCURRENCE OF TUMOIS}

Curtis has reported upon the frequency of occurrence of tumors of fowls based upon autopsies of 880 birds. Of these 79 , or 8.96 per cent had tumors. This percentage corresponds to 90 cases of tumors per 1000 birds. No significant difference was noted in frequency of occurrence of tumors, between birds which died from natural causes, and apparently normal birds which were killed. There is a significant correlation between age and the occurrence of tumors. Of the birds under $21 / 4$ years of age, only 7.37 per cent had tumors, while they were present in 19.17 per cent of birds over that age. In birds which died from natural causes, and showed tumor's, these were directly or indirectly the cause of death in from one-third to one-half the cases. There was a decided tendency for the association of hypertruphy of the liver, spleen or kidney, with the presence of tumors in other organs. The hypertrophy was apparently due to cell infiltration. Death often resulted from internal hemorrhage originating in the tumor, the underlying tissue or the hypertrophied liver or spleen. Data as to the character of the tumors were classified with reference to whether the tumors were cystic or of solid tissue structure. Those of cystic structure comprised 22.7s per cent and solid tumors constituted 74.65 per eent. Three cases fell in both classifications in that cysts were attached to solid tissue tumors. In females the genital organs were most frequently involved. In 37.76 per cent of all, the tumors were in the orary and 18.36 per cent involved the oviduct and oviduct ligament. Too few males were examined to warrant drawing conclusions. In most eases the tumors were confined to one organ. In 15 cases metastasis had evidently oceurred since tumors of similar nature were found in from two to four organs. 
Ehrenreich collected data on the frequency of the occurrence of tumors in fowls. Arrangement was made with a hotel to have sent to him the organs of all birds noticed to contain tumors. From nearly 2000 mature hens he obtained 7 malignant tumors of which 5 were surely carcinomata. Doubtless the hotel employees overlooked many small tumors. It should be noted that the birds examined were in apparent health and the discovery of carcinomata was purely accidental. Under more favorable conditions, the percentage of carcinomata would have been higher. Among 3000 pullets under a year old, no carcinomata were found, which confirms the belief that carcinoma is associated not with youth, but with maturity.

\section{CARCINOMA}

One carcinoma described by Ehrenreich was about the size of a hazel nut and had developed between the gizzard and the spleen. It was in contact with the upper end of the small intestine which showed stenosis at that point. The consistency was soft, the surface uneven and covered with shiny serosa. The color was gray and on section showed a few yellow points. Microscopic examination showed the tumor to be an adeno-carcinoma.

Two more highly differentiated adeno-carcinomata were obtained from the ovary. They were about the size of a small apple or a man's fist, were of solid consistency, had rough surfaces and were of a reddish gray color. In both cases a seeding of small metastatic tumors on the peritoneum had occurred.

Ehrenreich and Michaelis described three malignant tumors of the hen, of which two were adeno-carcinomata and one was a sarcoma-like tumor.

\section{SQUAMOUS CELL CARCINOMA}

Pick described a squamous cell carcinoma located beneath the floor of the mouth of a hen and which caused a marked deformation of the region. The subject was a hen about seven years old. The tumor was observed at New Year's as a small lump and by September had grown to a mass measuring 4 by 3 by $3 \mathrm{~cm}$. when the bird was killed. The tumor was located in the rear half of the floor of the oral cavity and bulged out on each side. It extended downwards to the sparsely feathered area between the wattles. The surface was smooth except for a small ulcerated spot near the beak. On section it was observed that the tumor was necrotic near the 
mouth cavity. Microscopic examination showed the tumor to be a typical squamous cell carcinoma.

Koch describes a squamous cell carcinoma (cancroid) of a hen. The subject belonged to the Brahma-Putra breed, which is noted for its longevity. The bird had been in the Berlin Zoological Gardens for seven years and was full grown when brought there. Thus it was concluded that the bird must have been at least eight year's old. The tumor was located in the roof of the oral cavity close to the back wall of the pharynx. It is to be noted that the other squamous cell carcinoma reported by Pick was located on the floor of the oral cavity and occurred in a seven year old hen.

According to Koch's description, the internal organs present no noteworthy features. On opening the oral cavity by a cut through the left commissure of the beak there is revealed a somewhat solid mass which completely fills the pharnyx and exerts such pressure on the larynx that it contains a depression corresponding to it. The surface of this yellowish mass is spotted with bright red blood clots which may well have caused death by strangling.

The yellow mass is so large that the disfigurement of the tracheal region would have been discovered in life but for the fact that it was covered with feathers. The tumor is very lightly attached and can be raised easily. It involves only soft tissue and no alteration of bone is observed. Microscopic examination shows the tumor to be a typical squamous cell carcinoma.

\section{CYSTO-ADENOMA}

Pickens has reported upon a cysto-adenoma in a fowl. The subject was a rather large Plymouth Rock and appeared to be in relatively good condition. The abdominal cavity was found to contain a pint of very thin straw colored fluid. After being collected in a flask, and after standing, a small amount of sediment was precipitated. The proventriculus, gizzard, liver, spleen, intestines and peritoneum were covered with numerous white lobulated tumor-like masses. These varied greatly in size, some measuring 1-2 $\mathrm{mm}$. in diameter while others measured 5 to $6 \mathrm{~cm}$. in diameter. They were either sessile or attached to the serosa by means of a very short stalk. The stalk seemed to be a continuation of the capsule of the nodule, and was easily broken, leaving the tumor free. The capsules of the nodules were smooth and resembled the peritoneum very closely. Many of the larger of these nodules contained small sacs 
or cysts attached to them. The cysts contained a small amount of serous fluid similar to that found in the abdominal cavity. Only one cyst occurred on a nodule. The proventriculus and gizzard showed only a few nodules. These were small varying from $2 \mathrm{~mm}$. to $2 \mathrm{~cm}$. in diameter. No cysts were found on the nodules of these organs.

Only a few nodules were attached to the liver and spleen. Those on the spleen were of the smaller variety and did not show cysts. There were several large nodules on the liver, the largest one measuring $6 \mathrm{~cm}$. in diameter and contained a cyst. The stalk of this tumor seemed to extend down through the capsule into the liver tissue proper. The serous coverings of the small and large intestines were affected alike and showed the attachments of many tumors of varying size. Section of a medium sized nodule showed it to contain several cysts containing thin, straw colored fluid. The cut surface of the tumor was white in color, tinged with pink. It was firm in consistency and numerous blood vessels were seen.

Histological examination of the tumors shows them to be made up of rather loose fibrous connective tissue stroma, relatively scarce in cells. Scattered irregularly through the stroma, and occupying approximately one-fourth of the area of the tumor are numerous alveoli, varying in size from a solid cord made of two or three epithelial cells to large cysts $5 \mathrm{~mm}$. in diameter, in the larger nodules. The alveoli are lined with epithelium which range from squamous to columnar in type. The alveoli vary greatly in shape. Some are irregularly oval, while others are much elongated and comparatively narrow, resembling the duct of a gland. Still others are found where numerous branching has taken place, giving the tumor the appearance of a papillary adenoma. The epithelium is, as far as can be determined, attached to a very thin basement membrane and the cells are only one layer thick. The connective tissue stroma of the tumor is exceedingly well supplied with blood vessels. The serous covering or capsules of the tumors are composed of rather dense fibrous connective tissue, which serves as the connection of the tumor to the serosa of the intestine. At the point of contact of the tumors with the liver, the liver cells have been replaced to a considerable extent with connective tissue stroma like that of the tumor. 


\section{FEATHER CYSTS}

Koch observes that feather cysts (cystomata pennifera) oceur in hens and geese, which are analogous to the dermoid cysts of mammals.

\section{IYMPHOMA}

Tyzzer and Ordway observe that lymphomata occur both with and without lymphatic leukemia. They regard the latter condition as due to the presence in the circulating blood of tumor cells having the characteristics of the cells of the lymphoid series. The only distinction made by them between a leukemic lymphoma and lymphoma with lymphatic leukemia is that in the former the tumor is extravascular while in the latter the tumor is intravascular. Tumors observed by them occurred as definite primary growth either with or without secondary nodules or were so disseminated that the site of origin could not be determined. Some of the tumors have a more or less alveolar structure while others grow diffusely by infiltrating normal structures. Examples are given of a few cases observed by Tyzzer and Ordway.

Lymphoma associated with lymphatic leukemia. Lymphomatous tumors are disseminated throughout the liver, the kidneys and the peritoneum; lymphatic leukemia. The subject is a hen at least one and possibly two years of age. The liver is greatly enlarged and presents many distinct rounded nodules of pale pink color distributed through its substance. Many of these nodules are apparent in the surface of the liver. Some present slightly depressed central areas, and elongated rounded borders. The largest measures $3 \mathrm{~cm}$. across. The spleen is several times its normal size and is rather soft. The cervical lymph glands are enlarged. The kidneys are enlarged and studded with rounded pink nodules, some of which are $4 \mathrm{~mm}$. in diameter. Distributed over various portions of the peritoneal surface are elevated plaques of tissue from which small blood vessels radiate into the surrounding normal tissue.

The tissue of this case being poorly preserved it is impossible to determine the finer histological features of the cells. It is apparent, however, that the nodules in the liver, the kidneys, and the peritoneum are composed of cells somewhat larger than the lymphoid cells of the circulating blood of the fowl and possess a more or less spherical, eccentrically situated nucleus. They correspond quite closely in their morphology to the plasma cell, and are evidently 
closely related to cells of the lymphoid series. The blood found in the vessels of various organs and tissues contains large numbers of these cells, so that the proportion of white cells in the circulating blood is very great. In addition to the nodules of tumor tissue found in the liver and kidneys, these organs are to a large extent infiltrated with tumor cells.

A case of lymphoma of the neck with metastasis to the lungs and the liver without lymphatic leukemia is described by the same writers. The cervical tumor appears as an oval flattened mass with an elevated rounded edge and a thick central crust. It measures 5 by $6 \mathrm{~cm}$. across and $2 \mathrm{~cm}$. in thickness. On section it is found to be composed of rounded cells which vary considerably as to size, and which contain rounded, somewhat irregular nuclei. The liver is greatly enlarged. The right lobe presents a rounded nodule, $1 \mathrm{~cm}$. in diameter consisting of tissue of similar appearance to that of the primary tumor. The entire liver is infiltrated with this tissue which appears to augment the size of the lobules and in places grows diffusely in areas 2 to $5 \mathrm{~mm}$. in diameter. The tumor involves both lungs, and only a small portion of one lung contains air. Near the roots of the lungs are two rounded nodules of tumor tissue. The tumor consists of cells with little supporting tissue. The latter consists for the most part of a delicate reticulum of connective tissue which apparently does not develop to any considerable extent within the tumor, but represents preëxisting tissue into which the tumor has grown. There are also a few coarser bands of fibrous tissue. The tumor is rather vascular throughout, and the connective tissue reticulum is more distinct around the vessels than elsewhere. The tumor cells vary in size. Many are no larger than the lymphocytes of the circulation but some are equal in size to large connective tissue cells. They are apparently not concerned in the formation of intercellular fibrils.

Another case reported presented a primary tumor of the nature of a lymphoma, situated on the neck, with secondary nodules on the sternum, in the lung and in the liver. The sternum and adjacent muscles are infiltrated with the tumor, but the tumor occurs as discrete nodules in the lung and liver. The spleen is not involved and the blood appears normal.

Warthin observed several cases of leukemic lymphocytoma in fowls in which there were observed tumor-like nodules and infiltration of lymphoid cells in all the organs, particularly in the liver, spleen, kidners, bone marrow, and hemolymph nodes. He observes 
that aleukemic and leukemic forms of lymphocytoma occur and is of the opinion that the two conditions appear to be genetically related if not different stages of the same process. He interprets the two conditions as malignant neoplasms.

\section{LEIOMYOMA}

Tyzzer and Ordway describe a leiomyoma of the mesentery of a hen. The specimen consists of a loop of hypertrophied intestine $30 \mathrm{~cm}$. long and 3 to $4 \mathrm{~cm}$. in circumference. Within the mesentery and covered on both sides by peritoneum is a large rounded tumor of firm, flesh-like consistence, which measures 7 by 5 by $5 \mathrm{~cm}$. This is of a general reddish pink color. On section the coloring is similar, and the surface is traversed by firm, glistening bands of somewhat lighter color. On scraping the surface, these stand out prominently and no soft tissue comes away. Between this tumor and the intestine is a tumor mass of similar character measuring 2 by $1.5 \mathrm{~cm}$. On histological examination the tumor is found to be composed of typical smooth muscle fibers running in various directions. Sections stained by the Van Gieson method for connective tissue show only a few small strands of fibrous tissue between the masses of smooth muscle fibers. The tumor is composed chiefly of smooth muscle fibers and is in many respects similar to the leiomyoma of mammals.

\section{OSTEOCHONDROSARCOMA}

Rous, Mrurphy and Tytler have described an osteochondrosarcoma. The fowl bearing the original growth was a Plymouth Rock hen in good condition and apparently about a year old. On the lower portion of the keel of the sternum was an irregularly spherical mass, so symmetrically disposed that the keel passed almost through its center. The tumor measured 6.6 by 5.7 by $4 \mathrm{~cm}$. The growth was smooth, nearly as hard as bone and the skin over it was slightly stretched but not firmly attached. The tumor was well encapsulated. In the gross specimen the sternal keel could be traced to the center of the growth, but here it was lost in a mass of red, spongr, bony tissue which radiated from it for a distance of one to one and one-half $\mathrm{cm}$. Peripheral to this, the tumor was hard and white with fine strands of opaque, fibrous tissue separating more translucent, homogeneous areas. 
Microscopically the capsule of the tumor was found to consist of fibrous connective tissue containing isolated muscle bundles. The growth itself was made up of a zone of what may be called prechondral tissue, enclosing and grading into a mass of hyaline cartilage through which ran the sternal keel. From this latter numerous irregular, bony trabeculæ radiated into the cartilage.

A brief recapitulation of the microscopic observations indicates that the outermost, youngest zone of the tumor is made up of cells of fibroblastic type scattered sparsely in a collagenous intercellular substance. In its deeper portions this tissue is undergoing a transformation to cartilage, its cells taking the character of cartilage cells, while the ground substance becomes homogeneous and basic staining. The original tumor is well encapsulated, and seems nearly, if not quite, stationary.

The growth is readily propagated in other birds by transplanting small bits of fresh, peripheral, neoplastic cartilage. By means of a trochar, the grafts are placed deep in the pectoral muscles. In growth resulting from the transplantation, cartilage is laid down, followed later by bone if the bird lives long enough. Death results from the tumor but rarely and in such instances the host gradually becomes emaciated. The tumor is not transferable to the pigeon.

The growth may also be induced by the filtrate of a Berkefeld filter. The tumor tissue is ground up with sterile sand. The resulting mass is suspended in Ringer's solution at blood heat and centrifugalized to free from tissue fragments. The supernatant fluid is passed through a Berkefeld filter of fine enough grade to retain $B$. fuorescens-liquefaciens. The percentage of tumors caused by the injection of filtrate is considerably higher when sterile infusorial earth is included. This material apparently causes a cell derangement favorable to tumor formation.

\section{SPINDLE CELL SARCOMA}

Rous observed a spindle cell sarcoma in a Plymouth Rock hen about fifteen months old. A large irregularly globular mass protruded from the right breast. It had developed slowly and without apparent involvement of the health of the host. The bird was anesthetized and nearly all of the growth was removed. When sliced it was found to have undergone a widespread coagulation necrosis at the center, but there was a rim of translucent, rather friable, yellowish pink tissue of glistening, finely striated surface. 
Autopsy of the bird reveals the presence of about 20 c.c. of a thin straw colored thuid in the peritoneal eavity. Attached to the lower margin of the liver, to the oblique membrane, and to the parictal peritoneum are many firm, pale yellow, ovoid globular nodules, the largest about $1 \mathrm{~cm}$. in diameter. On section these resemble the nodule in the left breast, except that in them the necrosis is irregularly distributed. At the pelvic region, where several of the masses have coalesced, softening and necrosis are extensive. No growths are visible in the other organs.

Microscopic examination of the tumor reveals it to be a spindlecelled sarcoma. In a typical section there are observed loose bundles of spindle cells coursing in every direction, and separated from the lesser blood vessels only by endothelium. Where such a bundle is cut transversely, the appearance is that of a group of round cells of varying sizes.

Transplantation of the tumor was accomplished by the use of fowls from the small, intimately related stock in which the growth appeared originally. Young chickens were more susceptible than adults. Market fowls of similar variety proved insusceptible as did pigeons and guinea pigs. During the first four generations, the tumor remained true to type and was infiltrative and destructive. Metastasis to the heart was observed once.

The tumor has been found to be transferable by the filtrate of a Berkefeld filter.

Rous has shown that ultraviolet light rapidly kills the cells of this transplantable sarcoma of the fowl without notably injuring the etiological agent associated therewith. The Roentgen ray has little effect on either cells or agent.

He observes that fowls manifest two sorts of resistance to the avian tumor, one directed against the implanted tumor cells as such, the other against the action of the etiological agent to cause a neoplastic change. In the individual fowl the two resistances appear to be independent of one another, though they may exist together or may both be absent. A recognition of them will perhaps explain some features in the biology of other tumors.

Rous and Murphy observe that variations have from time to time oceurred in the structure and behavior of this tumor. After a long series of transfers the growth frequently gave rise to fatal hemorrhages from its substance. In some of the later, rapidly growing tumors the cells tended to be spherical, showing only a very tardy and imperfect differentiation to the spindle form. A giant-celled 
form of the growth was sometimes met with. Despite their diversity, the tumors graded into one another and in the final analysis, all are to be considered as spindle-celled sarcomata.

Attempts to obtain an action of the etiological agent upon cells other than those it usually affects have failed, as have attempts to bring about changes in the histology of the sareomata by attenuating the agent.

Some of the lesser morphological variations in the sarcomata are undoubtedly due to local conditions of the host, and of the more important changes some have been associated with an increase in the malignancy of the growth. For others the determining conditions have yet to be discovered. On the whole the variations described are not more marked than those occasionally manifested by the transplantable mammalian tumors, and traceable to the changes in a single strain of tumor cells during their propagation in successive hosts. In mammals the ultimate reason for these changes is not known. In the case of the chicken tumor some of them are undoubtedly the expression of changes in the causative agent of the growth.

Rous and Lange describe a spontaneous chicken sarcoma. The subject was a mongrel brown Leghorn hen which was rendered lame by swellings on the left leg. The growth regarded as the primary one was located in the gizzard. It occupied nearly the whole right anterior portion of the organ and projected irregularly under a covering of mesentery in which were located several small nodules. The mass was roughly spherical and was about $4.3 \mathrm{~cm}$. in diameter. The tumor was surrounded by muscle and encroached on the gizzard cavity. The tumor had no capsule but was sharply defined by its pale pinkish white color as contrasted with the wine color of the muscle. The tumor was made up of many irregular, tightly compressed subdivisions separated by minute, irregular fissures, and bulged on the cut surface. A localized soft yellow area of necrosis was located to one side, but in general the growth was translucent and appeared sound though poorly vascularized. The liver was enlarged, congested and mottled with ill defined, pale areas. Two small stellate depressions making irregular gray areas in the parenchyma, were located on the surface of the organs. These proved to be composed of tumor tissue. The other viscera appeared normal.

An oblong smooth mass 4.5 by 3 by $3 \mathrm{~cm}$. was located in the extensor muscles of the left thigh. A similar one was located in the muscles below the knee. The upper mass was attached to the periosteum for about $2 \mathrm{~cm}$. above the patella and also to the joint capsule. 
A prolongation extended from it to the back of the joint. The lower growth was attached to the joint capsule but the bulk of it lay in the muscle. These growths were extremely firm, more so than the one in the gizzard. They were pinkish white in color and nearly bloodless. They consisted of finely striated tissue in part solid and in part divided into irregular lobuli, like the tumor in the gizzard. Some of the lobuli had a central, semigelatinous depression and in a few the center was yellow, firm and opaque, evidently necrotic. The growths were not encapsulated and were poorly defined from the muscle sheaths, periosteum and tendons which they involved.

The knee joint was full of translucent, gristly papillary proliferations attached to the capsule or joint surface. These were extensions from the growths on the outer surface of the capsule. Within the lower end of the femur and lying in the red marrow was a translucent tumor mass about $.4 \mathrm{~cm}$. in diameter. Tumors were also located in the muscle of the chest wall near the ribs, in the anterior muscles of the neck and elsewhere.

Microscopically, the tumor was found to consist of spindle-celled, sarcomatous tissue fissured and subdivided by many flattened sinuses, and often intracanicular in its growth.

Transplantation was carried through eight successive groups of fowls. The development of the first few series of transplantation tumors was very slow. They exhibited the histological structure of the original growth and showed the same tendency to form metastatic foci in the skeletal muscles. Later the tumor grew more rapidly and underwent a simplification of structure to that of a pure spindlecelled sarcoma. Plymouth Rock fowls proved to be quite as satisfactory hosts as the brown Leghorn breed in which the tumor occurred originally. The agent causing the tumor was capable of passing through a Berkefeld filter.

Görig reports multiple sarcomata in a Plymouth Rock hen about three or four years old. During life the subject displayed difficult breathing, was greatly emaciated as a result of difficulty in eating caused by two tumors, which hung from the lower side of the neck.

There is a tumor the size of a pigeon's egg suspended from the neck below the wattles. It is located in the subcutaneons connective tissue, has a solid consistency and the surface appears fincly roughened. The skin is for the most part attached only loosely to the tumor and only in a small spot is there a close union between the two. This is marked from without by an excoriated spot on the skin. The tumor measures 5 by $3 \frac{1 / 2}{\mathrm{~cm}}$. It is gray white in color 
and the cut section has a slight shiny appearance. There are observed whitish threads of connective tissue between which there are darker islands of tissue. Blood ressels seem somewhat scarce. No lesions are observed in the internal organs. Microscopic examination leads to the diagnosis of small spindle-cell sarcoma.

\section{LYMPHO SARCOMA}

Regenbogen has described a case of multiple sarcomata of the skin of a hen two years old. The subject is in good condition, displays good appetite and is active. A number of tumors are located on the head, neck, and back. These are of various sizes; one involving the left eye is $4 \mathrm{~cm}$. in diameter. The surface of this tumor is uneven. The center appears dark brown as a result of the presence of scabs and crusts. The periphery of the tumor shows the color of the skin and is thrown up in a circular ridge. The tumor extends above to the comb, below to the ear wattle, forward to the corner of the mouth and deep in the orbit. The other tumors move freely within the skin and are sharply defined from surrounding tissues. On the cut surface they are grayish white in color, smooth and fatty. The center of the surface of the larger tumors has undergone an ulcerative degeneration.

Microscopic examination shows round cells for the most part. Between these is a scanty and delicate reticular foundation structure. The vessels appear throughout as thin walled canals between the cell masses. From this it is seen that the tumor is a small round cell sarcoma, for instance, a lymphosarcoma.

Ziirn and Pauly have observed diffuse round cell sarcomata in the liver of hens, and Watson has reported upon a round cell sarcoma in the heart of a fowl.

\section{MELANOSIS}

Lewin described a case of general melanosis in a young slaughtered hen. The bird had coal black feathers, beak and legs. The comb was dark red, the tongue and oral mucosa, black. After removing the feathers, the whole body appeared bluish black, the musculature shone bright through the skin and after removal of the skin, appeared unaltered. The loose tissues between the muscle groups contained black patches of various sizes and shapes. After opening the body cavity the serous surface was observed to be completely black, as was 
the intestinal canal. Black patches were present on the gizzard and heart. After removing the flesh from the bones, the periosteum was observed to be wholly black as were also the cartilages of the joint surfaces. After scraping the periosteum the bone was seen to be black in places.

A specimen exhibiting generalized melanosis in a fowl is included in the pathological collection of the New York State Veterinary College at Cornell University.

\section{MIYXO-SARCOMA}

Tyzzer and Ordway describe a myxo-sarcoma of the thigh of a hen in part as follows. The tumor was situated on the outer aspect of the thigh of an adult hen, and appeared as a turban-shaped mass with a depressed central area and an elevated rounded edge. It measured $5 \mathrm{~cm}$. across and $2 \mathrm{~cm}$. in thickness. On section it was found to consist chiefly of a translucent, soft tissue almost gelatinous in consistence. The tumor is found to be composed of lobules of tissue which consist of cells widely separated from one another by a relatively large amount of intercellular material. The lobules are to a large extent limited by connective tissue which in places forms a definite capsule. In certain places, however, the tumor is without capsule of any sort and appears to be infiltrating the subcutaneous fat. The central portions of many of the lobules are necrotic, and the cells in the surrounding tissue are somewhat degenerated. The connective tissue around the lobules is loose and vascular in places, and, since there is in such places a marked infiltration with leucocytes, it resembles closely granulation tissue. In it are found large numbers of lymphoid and plasma cells, and large phagoertic cells.

The cells of this tumor present the characteristics of connective tissue cells, and are associated with intercellular material consisting in part of a fibrillar reticulum, in part of a homogeneous substance which reacts to staining reagents like mucin. The fibrillar material is probably closely related to collagin, although it is stained somewhat atypically with alum hematoxylin. The tumor is malignant in that it is rapidly growing, and in that it infiltrates normal tissues such as fat and nerves. Although blood ressels of considerahle size are found within many of the lobules, the tumor tissue is not ererywhere well vascularized, and is prone to necrosis. No metastases were found. 


\section{MYXO-FIBROMA}

Koch observed tumors in a partridge which elosely resembled avian tuberculosis. Both ceca were studded with numerous tubercules some as large as a pea. However the larger ones had a glazed appearance resembling eysts with gelatinous contents, while the smaller ones had an opaque, whitish gray appearance. Some of the larger tubercules showed a similar characteristic. Some of these were located in the intestinal wall protruding in the lumen as a hemisphere, without perforating the mucosa.

Microscopic examination showed the large tubercles to be myxofibroma and the smaller ones to be a pure fibroma.

\section{REFERENCES}

1. Curtis. Frequency of occurrence of tumors in the domestic fowl. $U$. S. Dep. Agr. J. Agr. Research, Vol. 5, 1915, p. 397.

2. Ehrenreich and Michaelis. Ueber Tumoren bei Hühnern. Ztschr. Krebsforch., Vol. 4, 1906, S. 586.

3. Ehrenreich. Weitere Mittheilungen über das Vorkommen malignes Tumoren bei Hühnern. Med. Klin., Berlin, Bd. 3, 1907, S. 614.

4. Görig. Multiple Sarkome beim Huhn. Deutsche tierärztl. Wchnschr., Bd. 8, 1900, S. 54.

5. Jones. A myxo-chondro-carcinoma of the testicle of a fowl. Rept. N. Y. State Vet. College, 1912-1913, p. 160.

6. Koch. Demonstration einiger Geschwulste bei Tieren. Verhandl. Deutsche Path. Gesellsch., Bd. 7-8, 1904, S. 136.

7. Lange. On certain spontaneous chicken tumors as manifestations of a single diesase. II. Simple spindle-celled sarcomata. J. Exper. M., Vol. 19,1914, p. 577.

8. Lewin. Ueber einen Fall von allgemeiner Melanose beim Huhn. Ztsch. f. Veterinärk., Bd. 22, 1910, S. 455.

9. Murphy and Rous. The behavior of chicken sarcoma implanted in the developing embryo. J. Exper. Med., Vol. 15, 1912, p. 119.

10. Petit et German. Le cancer de l'ovaire chez la Poule. Bull. Soc. Cent. de Méd. Vét. 'T. 63, 1909, pp. 341 and 386.

11. Pick. Zur Frage vom Vorkommen des carcinoms bei Vögeln: Grosser Plattenepithelkrebs des Mundhöhlenbodens bei einem Huhn. Berl. klin. Wchnschr., Bd. 40, 1903, S. 669.

12. Pickens. A cysto-adenoma in a fowl. Rept. N. Y. State Vet. Coll., 1913-1914, p. 261.

13. Regenbogen. Multiples Sarkom in des Haut eines Hahnes. Berl. tierärztl. Wchnschr., Bd. 19, 1907, S. 323.

14. Rous. Resistance to a tumor-producing agent distinct from resistance to the implanted tumor cells. J. Exper. M., Vol. 18, 1913, p. 416.

15. Rous. A transmissible avian neoplasm (Sarcoma of the common fowl). J. Exper. Mert., Vol. 12, 1910, p. 696. 
16. Rous. On certain spontaneous chicken tumors as manifestations of a single disease. I. Spindle-celled sarcomata rifted with blood sinuses. J. Exper. Med., Vol. 19, 1914, p. 570.

17. Rous and Lange. The characters of a third transplantable chicken tumor due to a filterable cause. A sarcoma of the intracanicular pattern. J. Exper. Med., Vol. 18, 1913, p. 651.

18. Rous and Murphy. Variations in a chicken sarcoma caused by a filterable agent. J. Exper. Med., Vol. 17, 1913, p. 219.

19. Rous and Murphy. On the causation by filterable agents of three distinct chicken tumors. J. Exper. Med., Vol. 19, 1914, p. 52.

20. Schlegel. Maligne Neoplasmen. Ztschr. f. tiermed., Bd. 17, 1913, S. 387 .

21. Tytler. A transplantable new growth of the fowl producing cartilage and bone. J. Exper. Med., Vol. 17, 1913, p. 466.

22. Tyzzer and Ordway. Tumors in the common fowl. J. Med. Research, Vol. 21, 1909, p. 459.

23. Warthin. Leukemia of the common fowl. J. Infect. Dis., Vol. 4, 1907, p. 369.

24. Watson. Malignant disease of a heart of a fowl. Vet. J., Vol. 9, 1904 , p. 182. 


\section{CHAPTER XXII}

\section{TOXICOLOGY}

It is a well established fact that considerable variations in tolerance to drugs of a toxic nature exist in animals of different species. While these variations have been pointed out in the case of mammalian species, little information is found in toxicologic literature on the toxicity to fowls of drugs or poisonous substances which may be of value in the treatment or control of avian diseases.

The experiments described in this chapter were undertaken by Gallagher for the purpose of determining the toxic doses for fowls of the more commonly used medicinal agents and of poisonous substances to which fowls not infrequently have access. No attempt was made to fix the toxic dose in certain instances where the substance on test proved non-toxic when given in comparatively large quantities. A few tests only were made with each substance. The results of these are summarized in the doses indicated under the name of each drug. In a few instances reference has been made to observations by other writers.

In each experiment, unless otherwise stated, fluids were administered directly into the esophagus or crop by means of a pipette and solids were given in gelatin capsules per os.

Medium sized healthy mature hens weighing between 3 and 4 pounds were usually employed. Those of larger size are designated. All doses were given while the crop contained a normal amount of food.

From the results of the experiments it may be concluded that in general fowls may be considered as having approximately the same susceptibility to toxic substances as medium sized dogs. They are more resistant than dogs to such substances as calomel, strychnine and tartar emetic, and less resistant to carbolic acid, salicylic acid and potassium cyanide.

In treating outbreaks of disease in fowls, it is of great advantage to employ the drinking water as a vehicle for medicinal agents. It is shown that fowls are not visibly affected by drinking solutions of bichloride of mercury 1-6000, carbolic acid 1-1000, permanganate 
of potash 1-500, and crude catechu 1-500 for periods of 18 to 21 days.

It is interesting to note that the crop not only serves as a reservoir for food, but that absorption through its wall is very rapid, symptoms appearing in from two to five minutes after the administration of such substances as ammonium chloride in solution, potassium cyanide and strychnine sulphate.

\section{AMMONIUAI CHLOMIDE}

Lethal dose. 60 gxains.

45 grains in solution ( 15 c.c. of 20 per cent solution).

Toxic dose. Same as lethal.

Non-toxic dose. 15 to 45 grains.

Experiments. (1) July 8, 1918, 2 р. м. Fowl received 60 grains of ammonium chloride. July 9. Fowl droopy, weak in legs, sitting. July 10. Same as July 9. July 11. Fowl died before 8 A. M. Autopsy notes. No lesions were apparent.

(2) February 20, 1918, 11.05 А. M. Fowl received 45 grains of ammonium chloride in 12 c.c. of water ( 15 c.c. of a 20 per cent solution). Stimulating effect observed almost immediately. Fowl had not been very active previously. After administration of the chloricle, bird began scratching energetically and singing more than the neighboring untreated fowls. Would drop wing on one side in imitation of a rooster and would crowd up against the side of the cage which separated her from another hen. Would peck at her through the cage. 11.20 A. Mr. Same as above. 11.25 A. Mr. Drinking considerable water. 11.30 A. Mr. Excitement has passed. 1.00 P. M. Fowl has a sleepy appearance. Bunches up somewhat when not disturbed. 4.30 p. M. Not active. Bunched up. February 21, 1918, 9 A. м. Fowl sleepy and bunched up. Stands ereet but wabbles when disturbed. 4.30 P. м. Fowl droopy. Bunched up. Feathers ruffled. February 22. Above described condition intensified. February 23. Fowl died before 8 A. .r. Autopsy notes. Crop filled with food. Lungs pale. Other organs apparently normal.

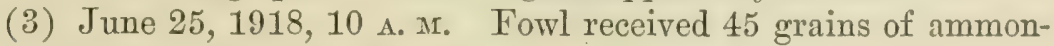
ium chloride. Result. No effect was apparent.

(4) February 28, 1918, 10.05 A. Mr. Fowl received 15 grains of ammonium chloride. Result. No effect was apparent. 
Lethal dose. 5 grains.

Toxic dose. 5 grains.

Non-toxic dose. 1 to 3 grains.

Experiments. (1) June 10, 1918, 10 A. x. Fowl received 5 grains of arsenious acid. 4.00 P. M. Fowl somewhat droopy. Droppings greenish. July 11, 9.00 A. x. Fowl droopy. Droppings greenish and watery. 12 м. Fowl weak. Comb dark. 1.30 P. м. Fowl dead. Autopsy notes. Food in crop and gizzard. Catarrhal exudate in the proventriculus. Internal membrane of the gizzard is necrosed and separated from wall at entrance of proventriculus. Contents of gizzard greenish. Duodenum shows a catarrhal condition and is pale in color. Contents of intestines are fluid and of greenish tinge. Liver is mottled. Rigor mortis is marked 1 hour after death.

(2) July 8, 1918, 2 P. xr. Fowl received 3 grains of arsenious acid. Result. No effect was apparent.

(3) June 25, 1918, 10.00 A. x. Fowl received 1 grain arsenious acid. Result. No effect was apparent.

Reinhardt fed .2 gram of arsenic and observed no srmptoms up to the sixtieth hour. He then gave 4 gram more and the hen died 40 hours after the last dose was administered. A second hen died $6 \frac{1}{2}$ days after receiving $.2 \mathrm{gram}$ of arsenic.

Reinhardt observes that arsenic poisoning in hens causes lesions which are characteristic with reference to location and character. The horny epithelial layer of the gizzard is raised from the underlying muscles by a gelatinous or sero-fibrinous exudate and in consequence is easily peeled off. The exudate lying beneath the epithelium, in places reaches a thickness of $1 \mathrm{~cm}$. Sometimes this exudate consists of a serous, yellowish, clear fluid, which spurts out when an incision is made in the tissues. In case the epithelial layer has been perforated, only a small amount of gelatinous exudate may be present. On removing the epithelial layer, highly reddened areas due to corrosive action, may be observed. The proventriculus shows no lesions. The mucosa of the crop is somewhat reddened, the vessels of the intestines are injected and the contents of the intestines are thinly fluid. The liver is usually yellowish brown in color and fragile, due to fatty degeneration. The fat, especially on the gizzard is somewhat edematous and soft. Frequently the fat is 
crange colored. The blood is well coagulated and brownish red in color.

\section{BICILLORIDE OF MERCURY (MERCURIC CIILORIDE, CORROSIVE SUBLIMATE)}

Lethal dose. 4 grains.

Toxic dose. 4 grains.

Non-toxic dose. 3 grains.

1-6000 solution as drinking water for 18 days without harm.

Experiments. (1) June 10, 1918, 10 A. M. Fowl received 5 grains of bichloride of mereury. 10.45 A. ‥ Spasmodic movements of the crop for several minutes. 11.45 A. м. Droopy. 2 p. м. Sitting. Legs weak. Drooping. 4 p. м. Sitting. Cannot stand. Marked depression. June 11, 1918, 9 А. м. Fowl found dead. Autopsy notes. No food in crop. Gizzard filled with food. Mucous membrane of dependent portion of crop is whitened and thickened as a result of coagulation necrosis. Proventriculus shows severe catarrhal condition. Mucosa at entrance to gizzard is necrosed. Membrane of posterior portion of gizzard is separated from the gizzard wall, the space being filled with clear fluid. M[ucosa of first third of intestine is exfoliated and remainder of small intestine shows a severe catarrhal condition. Other organs apparently normal.

(2) August 12, 1918, 2.20 P. xr. Four pound fowl received 4 grains of bichloride of mercury. 4.30 P. м. No change noted. August 13, 9 A. м. Legs very weak. Bird cannot walk. Sits down. Otherwise looks bright and normal. August 14, 9 A. м. Fowl cannot stand. August 15, 9 A. M. Fowl dead. Autopsy notes. Small amount of oats in crop. Crop wall is thickened, pale; mucosa is coagulated. Subcutaneous tissue surrounding crop and esophagus is infiltrated with a greenish gelatinous exudate. Proventriculus shows several hemorrhagic points on mueosa. Gizzard contains a considerable quantity of greenish colored food. Mucosa of small intestine is pale. Kidney is very pale and studded with minute white spots. Air sac membranes in abdominal eavity are thickened. The aldominal eavity contains 6 ounees of a thick somewhat riseid fluid with a slight greenish tinge.

(3) February 28, 1918, 10 A. M. Fowl received a tablet containing 3 grains of bichloride of mercury and 3 grains of ammonium chloride. Result, No effect was apparent. 
(4) May 27, 1918, 10 А. м. Gave fowl 1.4 grains of bichloride of mercury in feed. Fowl had not been fed for $2+$ hours, crop nearly empty. 3 P. м. Gave fowl another 1.4 grains of bichloride of mercury in feed. Result. No effect was apparent as a result of fowl consuming 2.8 grains of bichloride of mercury in feed in one day.

(5) June 14, 1918. Gave three fowls a 1-6000 solution of bichloride of mercury as drinking water. Fowls drank solution for a period of 18 days consuming about 2500 c.c. each. No other water was given. Result. No effect was apparent.

(6) June 12 to 14, 1918. Several fowls which had not received drinking water for 24 hours were given bichloride of mercury solutions of 1-2000 and 1-4000 as drinking water. Fowls tasted solutions and refused to drink. Solutions were clear. At intervals the solutions were again placed in the fowls' eages with the same result as above. After taking 1 to 3 swallows of solution the fowl shakes its head, rubs beak in the litter and elevates the feathers on the neck for a few moments. Gave fresh water and fowls drank eagerly.

Ward, adopting a suggestion by Ritzler, employed corrosive sublimate in the drinking water of a large flock of birds during an outbreak of fowl cholera, without harmful result. The period during which the sublimate was used to disinfect the water covered 16 days. For the first two days a solution of 1:1000 was employed. Since the fowls did not drink the solution readily, the bichloride was diluted to 1 part to 2000 parts of water.

BISMUTH SUBNITRATE

Non-toxic dose. 1/4 ounce +

Experiment. (1) February 20, 1918, 9.50 A. мr. Fowl received $1 / 4$ ounce of bismuth subnitrate. Result. No effect was apparent.

\section{CALCIUM OXIDE (QUICKLIME)}

Toxic dose. 11/4 drams.

Non-toxic dose. 1/2 dram.

Experiments. (1) August 15, 1918, 9.40 A. Mr. Gave 3 pound fowl $1 \frac{1}{4}$ drams of calcium oxide. August 16 . Fowl is somewhat droopy. Droppings are greenish in color. August 17. Fowl is dull in appearance. August 18. Fowl is dull in appearance. August 19. Fowl appears normal. 


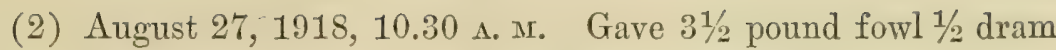
of calcium oxide. Result. No effect was apparent.

\section{CALOMEL (MERCURIOUS CHLORIDE)}

Non-toxic dose. 30 grains.

Experiment. (1) February 7, 1918, 10.30 A. M. Fowl received 30 grains of calomel. February 8, 9 A. M. Evidence of purging. Droppings greenish. Fowl has not been visibly affected otherwise.

\section{CARBOLIC ACID}

Toxic dose. 5 grains in solution (11 c.c of 3 per cent solution). 3.75 grains in solution (12.5 c.c. of 2 per cent solution).

Non-toxic dose. 2 grains in solution (13 c.c. of 1 per cent solution).

1-1000 solution as drinking water for 18 days.

Experiments. (1) August 12, 1918, 2.20 P. Mr. Five pound fowl received 11 c.c. of 3 per cent carbolic acid solution (5 grains carbolic acid). 2.22 P. M. Crop puffed out somewhat. 3.00 P. M. Fowl has shown some droopiness since receiving the solution. 3.45 p. x. Fowl apparently normal. 4.30 р. x. Fowl apparently normal. August 13. Fowl appears normal.

(2) February 2, 1918, 10 A. x. Three pound fowl received 12.5 c.c. of 2 per cent carbolic acid solution (3.75 grains carbolic acid). 11 А. M. Fowl shows dullness. 2 р. M. Fowl appears normal. February 3. Fowl is apparently normal.

(3) August 27, $1918,10.30$ А. мr. Gave 4 pound fowl 13 c.c. of 1 per cent carbolic acid solution ( 2 grains carbolic acid). Result. No effect was apparent.

(4) June 14, 1918. Gave three forvls a 1-1000 solution of carbolic acid as drinking water for a period of 18 days. Each fowl consumed about 2500 c.c. No other water was given. Result. No effect was apparent in any of the fowls.

(5) June 14, 1918. Several fowls were given carbolic acid solutions of $1-250$ and $1-500$ as drinking water. Fowls refused to drink the solutions. Gave fresh water and fowls drank eagerly. 
CASTOR OIL

Non-toxic dose. $6 \frac{1}{2}$ drams +

Experiment. (1) February 7, 1918, 10.30 A. M. Fowl received $61 / 2$ drams of castor oil. February 8, 1918. 9 A. м. Evidence of moderate purging. Droppings greenish. Fowl was not visibly affected otherwise.

\section{CATECHU (CRUDE)}

Non-toxic dose. 1-500 solution +

Experiment. (1) June 10, 1918. Gave fowl a 1-500 solution of crude eatechu as drinking water. Fowl drank solution freely. July 1, 1918. Fowl drank 3000 c.c. of above solution in 21 days. No other water was given. No constipation was observed. No change in fowl was apparent.

CHLORIDE OF LIME

Non-toxic dose. $3 / 4$ to $1 \frac{1 / 2}{2}$ drams.

Experimente. (1) August 15, 1918, 10.30 A. ‥ Five pound fowl received $1 \frac{1}{2}$ drams of chloride of lime. Result. No effect was apparent.

(2) August 13, 1918, 2.30 P. M. Five pound fowl received 3/4 dram of chloride of lime. Result. No effect was apparent.

\section{COPPER SUlPhATE (BLUE STONE)}

Lethal dose. 20 grains.

15 grains in solution.

Toxic dose. Same as lethal.

Non-toxic dose. 5 to 15 grains.

Experiments. (1) July 8, 1918, 2 р. м. Fowl received 20 grains of copper sulphate. 4 р. м. No change apparent. July 9. Fowl droopy. Sitting. July 10. Same as July 9. July 11, 9 А. M. Fowl dead. Autopsy notes. Pharynx and esophagus show coagulation of the mucosa. Mucosa of crop exfoliated. Crop filled with water and greenish catarrhal exudate. Lower esophagus shows coagulation necrosis of mucosa. Proventriculus shows severe catarrhal gastritis. Catarrhal enteritis. Entire intestine is filled with greenish catarrhal exudate. 
(2) February 2, 1918, 10 A. M. Fowl received 15 grains of copper sulphate in solution (10 c.c. of 10 per cent solution). Fowl drank water continuously for 15 minutes then became restless for about three minutes. 12 м. Dull. 2 р. м. Dull. 4.30 r. м. Dull. February 3, 1918, 9 А. м. Fowl is droopy. 9.30 А. м. Convulsions developed and fowl died. Autopsy notes. Crop normal. Distended with water. Considerable food in erop and gizzard. Mucosa of proventriculus intensely inflamed. Horny membrane of gizzard loosened near opening of proventriculus. IIemorrhagic points in submucosa. Catarrhal enteritis is quite marked. Intestine contains considerable bluish fluid. Mesenteric fat and peritoneum are petechiated. Heart is in systole.

(3) June 25, 1918, 10 А. .. Fowl received 15 grains of copper sulphate. Result. No effect was apparent.

(4) February 28, 1918, 10.10 A. ג. Fowl received 5 grains of copper sulphate. Result. No effect was apparent.

\section{ERGOT. F. E.}

Non-toxic dose. $2 \frac{1}{2}$ drams F. E. equivalent to $2 \frac{1}{2}$ drams of ergot. Experiment. (1) February 8, 1918, 10.30 A. ‥ Fowl received $21 / 2$ drams of fluid extract of ergot with $2 \frac{1}{2}$ drams of water. Result. No effect was apparent.

\section{FERROUS SULPHATE (COPPERAS)}

Non-toxic dose. 30 grains +

Experiment. (1) February 7, 1918, 10.30 A. м. Fowl received 30 grains of ferrous sulphate in solution (10 c.c. of 20 per cent solution). Result. No effect was apparent.

IPECAC. F. E.

Lethal dose. 1 to 2 drams.

Toxic dose. 1 dram.

Non-toxic dose. $1 / 2$ to $3 / 4$ dram.

Experiments. (1) August 19, 1918, 1.10 P. Mr. Gave 41/2 pound fowl 2 drams fluid extract of ipecac. August 20. No apparent change. August 21, 9 ․ з. Greenish fluid droppings. August 22, 9 A. x. Fowl dead. Autopsy notes. Crop and gizzard filled with food. Mucosa of crop pale, slightly thickened. Lower esophagus 
and proventriculus show a catarrhal condition. Several hemorrhagic points in proventriculus. Small area of internal lining of gizzard at entrance of proventriculus separated from gizzard wall. Mucosa of duodenum pale. Liver pale.

(2) August 19, 1918, 1.10 р. м. Gave 3 pound fowl 11/4 drams fluid extract of ipecac. August 20,9 A. x. Fowl dead. Autopsy notes. Some food in crop. Proventriculus shows a catarrhal condition. Hemorrhagic near entrance to gizzard. Membrane of gizzard easily separated near opening of proventriculus. Liver is banded with light and dark stripes.

(3) August 27, 1918, 10.30 A. M. Gave 41\%2 pound fowl 1 dram of fluid extract of ipecac. August 28. No change apparent. August 29. Fowl dull. August 30. Fowl dead at 9 A. M. Autopsy notes. Crop and gizzard filled with food. Mucosa of proventriculus thickened and catarrhal. Submucosa of gizzard near proventricular opening hemorrhagic. Liver pale in spots and streaked with darker bands. Heart in systole.

(4) August 12, 1918, 2.20 P. M. Gave 5 pound fowl 1/1/4 drams fluid extract of ipecac. August 14, 9 A. M. Fowl has shown no change. August 15. Somewhat dull. August 16. Same as August 15. August 17. Apparently normal.

(5) August 30, 1918, 10.30 A. x. Gave 4 pound fowl $3 / 4$ dram of fluid extract of ipecac. Result. No effect was apparent.

(6) August 30, 1918, 10.30 A. M. Gave 4 pound fowl 1/2 dram of fluid extract of ipecac. Result. No effect was apparent.

\section{LEAD OXIDE (IITHARGE)}

Non-toxic dose. 11/4 drams.

Experiment. (1) August 15, 1918, 9.40 A. M. Five pound fowl received $1 \frac{1}{t}$ drams of lead oxide. Result. No effect was apparent.

\section{MAGNESIUM. SULPHATE}

(Epsom Salts)

Non-toxic dose. 1 dram in solution +

Experiment. (1) February 7, 1918, 11 А. м. Fowl received 1 dram of Epsom salts in 4 $1 \frac{1}{2}$ drams of water. February 8, 9 A. м. Evidence of moderate purging. Droppings fluid and brownish. Fowl was not visibly affected otherwise. 
MIALE FERN. F. E.

Toxic dose. $2 \frac{1}{2}$ drams, F. E.

Non-toxic dose. 11/4 drams, F. E.

Experiments. (1) April 25, 1918, 11.15 A. м. Fowl received

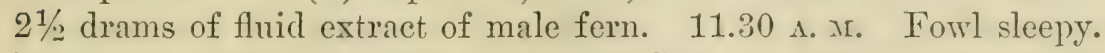
Droopy. 11.45 . А. м. Fowl sleepy. Sitting. 12 м. Legs very weak. 1 P. Mr. Very weak. Can raise itself on its feet with difficulty. 2.45 p. x. Sitting. Has great difficulty in attempting to stand. 4 P. M. Standing but legs are still weak. April 26, 1918, 9 A. Mr. Fowl has recovered.

(2) July 8, 1918, 2 P. м. Fowl received 1/1/4 drams of fluid extract of male fern. Result. No effect was apparent.

\section{PHOSPHORUS}

Poisoning with this substance may occur as a result of eating rat poison. The writers have observed a case of phosphorus poisoning induced by eating the debris left after a display of fireworks among which was fine gravel employed in "torpedoes."

Birds exhibit depression, weakness, trembling, diarrhea, thirst and sometimes emphysema of the skin. Death may occur within a period varying from one hour to several days.

At autopsy, care should be taken in opening the crop, proventriculus and gizzard in looking for the so-called phosphorus vapor which appears as a transient cloud on opening the organs or when the contents are moved. The phosphorus in the crop and gizzard may be detected by the characteristic odor and by faint luminosity when in the dark, especially if the material is rubbed. The more pressure exerted, the more luminous it appears. The most marked lesions occur in the proventriculus and are characterized by hemorrhages and erosions in the mucosa. Inflammation is also present in the rpper portions of the intestines. Ecchymoses may be present on the heart and in various organs. In cases of long standing there may be fatty degeneration of the liver and other organs, and yellow colored liver. 


\section{POTASSIUM CYANIDE}

Lethal dose. 1 to 2 grains.

Toxic dose. $1 / 10$ to $1 / 2$ grains.

Experiments. (1) February 20, 1918, 10.15 A. M. Gave fowl a 2 grain crystal of potassium cyanide per os. Symptoms appeared in 4 minutes. Fowl had difficulty in maintaining its balance. Dropped to the floor. After several minutes it fluttered around the cage for about five seconds, went down again, became comatose and was dead in 12 minutes after swallowing the cyanide.

(2) February 20, 10.30 A. M. Gave fowl a 1 grain crystal of potassium cyanide per os. Symptoms appeared in 2 minutes. Fowl became droopy, stood for several minutes with head dropped to floor (limber neck), fell to floor in a comatose state and was dead 13 minutes after swallowing the cyanide. Did not struggle at any time.

(3) July 8, 1918, 2 r. м. Gave fowl a 1/2 grain erystal of potassium cyanide per os. 2.02 P. xr. Fowl jumped suddenly striking top of cage. Stands very erect. Breathing more rapidly. Mouth open. 2.05 P. x. Unsteady on legs. Wings drooped. Distressed expression. 2.20 P. M. Fowl lying down. Continues breathing through mouth. 3 r. M. Same as above. Fowl can stand when forced up. 4 P. M. Fowl has recovered.

(4) February 28, 10.15 А. .. Gave fowl $1 / 5$ grain of potassium cyanide in gelatin capsule per os. 10.23 A. M. Fowl breathing rapidly through the mouth. 10.25 A. x. Wings drooping. Has attempted to pass droppings four times, each time a very small amount has been passed. 10.45 A. M. Same as above. Getting sleepy. 11 A. M. Same as above. Sleepy. 12 Mr. No change except that breathing is not as rapid. 1.30 P. Mr. Fowl has recovered.

(5) August 12, 2.23 P. x. Gave 3 pound fowl 1/10 grain potassium cyanide in small piece per 0s. 2.26 P. м. Breathing rapidly. 2.30 р. м. Sitting. 3 р. м. Has depressed appearance. Drowsy. Half sitting posture on being placed on feet. Sits again. Breathing not so rapid. 3.45 р. м. Standing. Some droopiness apparent. Breathing is normal and fowl is recovering. 4.30 Р. м. Apparently normal. August 13,9 A. м. Fowl is apparently normal.

\section{POTASSIUM PERMANGANATE}

Lethal dose. 30 grains.

Toxic dose. 30 grains. 
Non-toxic dose. 15 grains.

15 " in solution.

1-500 solution as drinking water.

Experiments. (1) June 25, 1918, 10 A. x. Gave fowl 30 grains of potassium permanganate. 4.30 P. Mr. No symptoms noticed. June 26, 9 A. x. Fowl died during night. Autopsy notes. Crop filled with oats and some water. Dependent portion of erop charred and softened. Apparently the mucous membrane was eaten through. Submueosa blackened, also skin on lower surface of crop and adjacent breast. Thick coating of moist black material on lower mucosa of crop. Probably remains of permanganate. Several blood clots in crop. Remainder of crop mucosa normal. All other organs normal. Permanganate had not left crop as far as could be determined. Apparently hemorrhage had occurred in subeutaneous tissue and blood had been oxidized.

(2) February 2, 1918, 10 A. м. Gave fowl 15 grains of potassium permanganate in solution (30 c.c. of $3 \frac{1}{3}$ per cent solution). Result. No effect was apparent.

(3) July 8, 2 P. м. Gave fowl 15 grains of potassium permanganate. Result. No effect was apparent.

(4) June 10, 10 A. M. Gave fowl a 1-500 solution of potassium permanganate as drinking water. Fowl drank solution freely. July 1. Fowl drank about 3000 c.c. of above solution in 21 days. No other water was given. Result. No effect was apparent.

\section{ROSE CHAFERS}

Lamson has observed poisoning of chickens due to eating rose chafers (Macrodatylus subspinosus).

When opportunity affords, chickens eat rose chafers ravenously. Within an hour after feeding the birds assume a dozing attitude. Later, weakness is apparent and death usually oceurs within $2 t$ hours. Convulsions occur in less than five per cent of the cases.

At autopsy the crop is usually found to be so full of insects as to give the impression that death has been due to "erop bound "but no abnormal condition of other organs is observed.

Feeding experiments have shown that 15 to 20 rose chafers are sufficient to kill a chicken one week old. From 25 to 45 are necessary to kill a three weeks old chicken. Ten weeks about marks the age limit of suseeptibility. The damage is not merely mechanical, for an extract of erushed rose chafers in distilled water, after filter- 
ing, will cause death when fed to chickens. The rose chafers apparently contain a toxin affecting the heart.

Preventive measures should consist in keeping chickens on mowed fields and away from grape vines and flowering shrubs during the month when rose chafers are prevalent.

SALICYLIC ACID

Lethal dose. 30 to 75 grains.

Toxic dose. 30 grains.

Non-toxic dose. 15 grains.

Experiments. (1) June 10, 1918, 10 A. M. Gave fowl 75 grains of salicylic acid. 11.30 A. M. Wings dropped. Fowl sleepy. Droopy. 12 м. Condition growing worse. 1.30 p. м. Bird dead. Autopsy notes. Crop filled with oats. No trace of the three gelatin capsules. At least half of the salicylic acid administered remains in the esophagus. MLucous membrane of mouth, esophagus, crop and lower esophagus is white from action of the salicylic acid. Protoplasm is apparently coagulated. Tot so severe in lower esophagus and erop as in upper esophagus and mouth. No other lesions apparent.

(2) June 25, 1918, 10 A. M. Gave fowl 30 grains of salicylic acid. 4 r. x. Fowl droopy. June 26, 9 А. x. Fowl lying on side in comatose condition. 2.30 P. м. Fowl died. Autopsy notes. Crop filled with food and some white material probably salicylic acid. Dependent portion of wall of crop thickened, wrinkled and coagulated, white in color. Mucosa of lower esophagus and proventriculus whitened. Intestine mildly hemorrhagic throughout its length. Wall congested. Liver dark, capsule thickened in one place. Spleen soft. Ovary shows $B$. pullorum infection.

(3) July 8, 2 r. M. Gave fowl 15 grains of salicylic acid. Result. No effect was apparent.

\section{SANTONIN}

Non-toxic dose. 5 to 15 grains.

Experiments. (1) June 25, 10 A. м. Gave fowl 15 grains of santonin. Result. No effect was apparent.

(2) June 10, 10 A. Mr. Gave fowl 5 grains of santonin. Result. No effect was apparent. 


\section{SODIUM CHLORIDE (COMMON SALT)}

Lethal dose. $2 \frac{1}{2}$ drams.

$$
2 \frac{1}{2} \text { drams in solution. }
$$

Toxic dose. Same as lethal.

Non-toxic dose. $1 \frac{1}{4}$ to $1 \frac{3}{4}$ drams.

Experiments. (1) February 2, 10 A. Mr. Gave fowl 21/2 drams of sodium chloride in solution ( 40 c.c. of 25 per cent solution). 11 A. M. Fowl is dull. 12 мr. Same. 2 p. M. Same. Droopy. Sleepy. 4.30 p. x. Fowl is sitting. Sleepy. February 3, 9 ^. м. Fowl found dead. Autopsy notes. Considerable food in crop and gizzard. Crop, proventriculus and gizzard are normal. Small intestine normal. Rectum slightly inflamed. Liver darkened. Kidney congested. Heart is systole.

(2) June 25,10 A. м. Gave fowl 21/2 drams of sodium chloride. June 26, 9 A. м. Fowl droopy. 1 р. м. Paralyzed. 4 р. м. Paralyzed. Shows condition known as wry neck. June 27, 9 A. м. Paralyzed. Lying on side. Wry neck. 11 A. M. Died. Autopsy notes. Crop gorged with food. Mucosa of crop white, cooked appearance, thickened. Severe catarrh of proventriculus, tenacious mucous exudate. Horny membrane of gizzard easily removed. Duodenum shows slight congestion. Catarrh of duodenum. Liver darkened.

(3) July 8, 2 р. м. Gave fowl 13/4 drams of sodium chloride. Result. No effect was apparent.

(4) February 28, 10 A. x. Gave fowl 1 1/4 drams of sodium chloride. 10.30 A. м. Fowl shows a moderate thirst. Result. No effect was apparent.

Suffran observes that fatal poisoning is induced in the hen by a dose of 4 grams of common salt per kilo of body weight, while the pigeon succumbs to 5 grams per bird.

The clinical picture of intoxication by salt gives the impression that it acts as a poison of the muscles. The gradual weakening of the contractile power induces progressive difficulty in walking and at last falling down. It is not a true paralysis for sensation and movement continue until death. Pricking, and the point of a thermo cautery produce response by contraction. The weakening of the muscles of respiration causes a progressive asphyxia which leads to death.

The toxic action of salt is exereised also in a certain measure on 
the nervous centers. A proof of that influence is seen in the phenomena of somnolence which dominate the morbid scene, in the hyperesthesia which is manifested by the transitory crises and last by the interference with locomotion.

The lesions are chiefly in the digestive canal. The buccal mucosa is covered with a yellowish brown layer which is viscid and adherent. When this is removed, marked congestion is seen, with hemorrhages at some points. The mucosa of the crop shows traces of a violent irritation with numerous hemorrhages and superficial ulceration. The mucosa of the esophagus shows general congestion with superficial crosions. The intestinal mueosa is hyperemic, hemorrhagic and ulcerated. The principal visceral organs such as liver, spleen, kidneys, lungs, heart and nervous centers such as brain and cord show more or less marked congestive lesions. The blood possesses a characteristic bright red color.

Edwards found that doses of salt as high as 2.5 grams per kilogram of body weight administered to pigeons by injecting into the crop, produced no effect. A dose of 4.5 grams per kilogram of body weight eaused great depression, followed by death in eighteen hours. $\Lambda$ similar dose of 3.93 grams per kilogram cansed death in twentythree hours. No lesions other than acute congestion of the mucous membrane of the lower portion of the esophagus were observed. Only about 25 per cent of the salt was absorbed from the crop.

SODIUM NITRATE

Lethal dose. $2 \frac{1 / 2}{d r a m s .}$

Toxic dose. 11/4 drams.

Non-toxic dose. 1/2 dram.

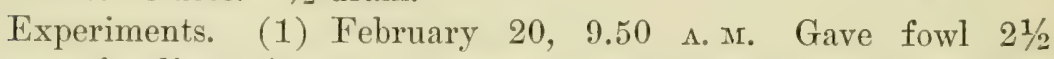
drams of sodium nitrate. $11 \mathrm{~A}$. M. Fowl dull and droopy. $12 \mathrm{Mr}$ Fowl shows increased dullness, droopiness and sleepiness. Sitting. 1 P. M. Fowl is wide awake but dull. Is in a sitting posture. Legs paralyzed, cannot stand. 3 p. x. Fowl lying on its side, cannot move, legs paralyzed. Greatly depressed. Death appears imminent. 4.30 p. м. No change from above. February 21, 9 А. м. Fowl died during night. Antopsy notes. Crop wall and skin covering it dehydrated. These membranes were transparent, dry and tough. Proventriculus shows marked catarrhal exudate. Inner membrane of gizzard separated from submucosa. Considerable fluid beneath inner membrane. Catarrhal enteritis. Duodenal mucosa congested 
somewhat. Liver dark. Spleen pale. Pancreas enlarged and infiltrated. Heart is systole. Considerable food in crop.

(2) February 28, 1918, 10 ^. м. Gave fowl 11/4 drams of sodium nitrate. 1.30 P. x. No change is apparent. 3.30 r. x. Fowl bunched up and sleeping. On being aroused displays marked thirst. Becomes droopy and sleepy again. 4.30 P. M. Fowl droopy and shows thirst. March 1,9 A. M. Fowl quiet. Sits down. Comb and wattles dark red. 12 ‥ Same as above. 1.30 p. м. Fowl paralyzed, no use of feet, prostrated. 3.30 г. м. Same as above. Comb blackening at the tips. 4.30 P. M. Same as above. March 2, 9 А. .. Fowl up, normal in appearance but quiet on being handled. Comb a bright red. 3 р. x. Fowl apparently normal.

(3) June 25,10 A. M. Gave fowl $1 / 2$ dram of sodium nitrate. Result. No effect was apparent.

\section{STRYCHNINE SULPHATE}

Lethal dose. 2 grains to $3 \frac{1 / 2}{2}$ pound fowl.

Toxic dose. 2 to 3 grains to 5 pound fowl.

1.5 grains to 3 pound fowl.

8 c.c. F. E. nux vomica $=1.2$ grains to 3 pound fowl.

Non-toxic dose. 2 grains to 5 pound fowl.

Experiments. (1) August 12, 2.22 P. Ar. Gave 31/2 pound fowl 2 grains of strychnine sulphate in gelatin capsule per os. $2.30 \mathrm{P}$. $\mathrm{x}$. Breathing more rapidly. Some unsteadiness of legs. 2.31 P. xr. Convulsion lasting 10 seconds. Fowl down on side. 2.32 P. Mr. Slight convulsion. Rapid breathing. 2.35 P. Mr. Slight convulsion. 2.36 P. м. Fowl stretched out at length. Rapid shivering of legs. 2.40 r. x. Fowl dead. Autopsy notes. Fowl stiff. Considerable fluid in crop. Heart in systole. Liver slightly eongested. Comb, wattles and face darkened.

(2) August 12, 2.23 P. м. Gave 5 pound fowl 3 grains of strychnine sulphate in gelatin capsule per os. 2.45 P. . I. Breathing rapidly. Unsteady on legs. 2.50 P. м. Sitting. 2.55 Р. Mr. Standing. Breathing 180 per minute. 3.45 P. x. Breathing considerably decreased. Some unsteadiness on legs through lack of normal powers of balance. 4.30 P. Mr. Appears improved. August 13, 9 A. м. Fowl's legs straddled. Cannot stand up. When disturbed clonic spasms lasting 4 or 5 seconds are set up. August 14, 9 А. .. Fowl paralyzed. August 15, 9 А. м. Fowl stands fairly erect for a short time but is unsteady on its feet. Diarrhea present. 
August 16. Fowl stands but is unsteady on feet. Much improved. August 17, 9 A. M. Fowl appears normal.

(3) July 8, 1.55 P. M. Gave 5 pound fowl 2 grains of strychnine in gelatin capsule per os. 2.10 P. M. Fowl unsteady on feet. 2.15 P. м. Sways over backwards. Weak in legs. 4 P. мr. No other change observed. Fowl apparently normal.

(4) February 19, 2.10 P. 1r. Gave fowl 1.5 grains of strychnine sulphate in gelatin capsule per os. 2.30 P. мr. Fowl suddenly affected. Delay due to slowness of liquefaction of the capsule. Fowl staggers, legs spread, bird drops on her side. Several severe spasms in first 5 minutes, breathing rapidly. 2.40 P. xr. Fowl sleepy, dozes for a moment and then starts suddenly, breathing rapidly, no spasms. 3 р. мr. Breathing less labored, fowl still prostrated. 4 P. мr. Bird in sitting position. Can rise slightly from the floor but cannot stand erect. Breathing normal and fowl appears bright and normal other than the leg weakness. February 20,9 A. M. Fowl is apparently normal except for incoordination of movement. Unsteady on its feet and lifts them higher than normal in walking. February 21. Fowl improving in ability to control legs but still unsteady. February 23. Fowl apparently normal.

(5) February 8, 10.30 А. м. Gave fowl 8 c.c. of fluid extract of nux vomica ( 1 per cent strychnine) with 8 c.c. $\mathrm{H}_{2} \mathrm{O}$ by tube into crop $=1.2$ grains of strychnine. 10.35 A. M. Fowl nervous. Breathing very rapidly. 10.40 A. M. Fowl wabbles, difficult to maintain balance, legs weak, bird does not stand erect. Severe spasm of muscles of body and wings lasting 10 seconds. 10.50 A. M. Fowl in sitting position from leg weakness or partial paralysis, breathing rapidly. Has had three violent convulsions. Comb and wattles redder than normal. 11 А. м. Breathing much slower. No more spasms. Bird is recovering. 11.30 A. M. Fowl can stand. 12 M. Fowl appears normal. 4.30 P. x. Fowl normal.

(6) June 25, 10 A. M. Gave 5 pound fowl 2 grains of strychnine in gelatin capsule per os. No effect apparent except a slight increase in activity for several moments one hour after administration.

Schneider determined the therapeutic and lethal doses for strychnine nitrate for various birds. His results presented in tabular form below refer to doses in milligrams per kilo of body weight. 


\begin{tabular}{lcccc} 
& \multicolumn{2}{c}{ Subcutaneous } & \multicolumn{2}{c}{ Internal per os. } \\
& Therapeutic & Lethal & Therapeutic & Lethal \\
Geese & .4 & $1.0-2.0$ & .6 & 2.5 \\
Ducks & $.5-.6$ & $1.0-1.1$ & $1.5-2.0$ & $3.0-4.5$ \\
Hens & 1.0 & $3.0-5.0$ & $2.0-3.0$ & $30.0-140.0$ \\
Pigeons & $.5-.75$ & $1.0-1.5$ & 6.0 & $8.5-11.0$
\end{tabular}

It will be observed that pigeons are least susceptible to internal therapentic doses, while hens, ducks and geese are more so. However, with reference to lethal doses per os, hens are most resistant, and pigeons, ducks and geese less so.

The therapeutic doses expressed above in milligrams per kilo of body weight, are given below on a basis of milligrams per bird.

$\begin{array}{lcc} & \begin{array}{c}\text { Therapeutic doses } \\ \text { Subcutaneous }\end{array} & \text { Per os. } \\ \text { Geese } & 1.4 & 2.16-2.22 \\ \text { Ducks } & .7-.96 & 2.5-3.8 \\ \text { Hens } & .7-1.5 & 2.0-5.0 \\ \text { Pigeons } & .16-.26 & 2.0-2.4\end{array}$

\section{SULPHUR}

Non-toxic dose. 1/4 ounce +

Experiment. (1) February 20, 9.45 A. M. Fowl received 1/4 ounce of sulphur. Result. No effect was apparent. Fowl remained as active as it was previous to administration of the sulphur.

\section{TARTAR EMETIC}

Lethàl dose. 10 to 15 grains.

Toxic dose. 10 grains.

Non-toxic dose. 5 grains.

Experiments. (1) February 20,9.40 A. M. Gave forvl 15 grains of tartar emetic. 12 м. No special change except that the bird is quieter than normal. 1 p. м. Bird quieter than normal. Sits down a good deal. 4.30 P. x. Quiet but shows no special symptoms. February 21, 9 A. м. Fowl died during the night. Autopsy notes. Comb normal. Heart in systole. Crop contains considerable food. Proventriculus shows catarrhal condition. Intestine catarrhal. Duodenum petechiated. Heart petechiated. Liver pale. Kidneys congested.

(2) June 25, 10 А. x. Gave fowl 10 grains tartar emetic. June 26. No apparent change. June 27. No apparent change. June 
28, 9 A. x. Fowl dead. Autopsy notes. Considerable food in crop. Mucosa of dependent portion of crop shows a number of small necrotic patches and erosions. Mrucosa of duodenum hemorrhagic. Liver congested and pale in spots. Heart in systole.

(3) February 28, 10.15 A. .. Fowl received 5 grains of tartar emetic. Result. No effect was apparent.

\section{TURPENTINE}

Non-toxic dose. $2 \frac{1}{2}$ drams +

Experiment. (1) February 7, 10.30 A. M. Fowl received 2/1/2 drams of turpentine with $2 \frac{1}{2}$ drams of linseed oil. Result. No effect was apparent.

\section{REFERENCES}

1. Edwards. Salt poisoning in pigs and poultry. Jour. Compar. Path . and Ther. Vol. 31, 1918, p. 40.

2. Gallagher. Experiments in avian toxicology. J. Am. Vet. Med. Ass., Vol. 7, 1919, p. 337.

3. Lamson. The poisonous effects of the rose chafer upon chickens. Science, n. s. Vol. 43, 1916, p. 138.

4. Reinhardt. Beiträge zur Kenntniss der Geflügelkrankheiten. Berl. tierärztl. Wchnschr., Bd. 30, 1914, S. 214.

5. Schneider. Toxikologische Versuche mit Strychninum nitricum bei Gänsen, Enten, Hühnern und Tauben. Monatschr. f. prakt. tierh., Bd. 11, 1899-1900, S. 245.

6. Suffran. Sur l'empoisonnement des volailles par le sel de cuisine. Rev. Gén. Méd. Vét., T. 13, 1909, p. 698. 


\title{
CHAPTER XXIII
}

\author{
SURGICAL DISEASES
}

\section{ANESTHETIZING FOWLS}

Occasions demanding the administration of a general anesthetic are limited largely to serious operations undertaken in connection with the study of the functions of certain organs. Pearl and Surface have pointed out that by the ordinary method of administration of an anesthetic, ninety per cent of the subjects will die under the anesthetic. If less of the drug is administered, reflex excitability is not lost, and the bird will struggle. It is likely that the explanation of the high mortality lies in the fact that the air sac system of birds furnishes opportunity for the storage of a large amount of anesthetic which is wholly absorbed in a short time and not eliminated by expiration as in mammals. The excessive amount of anesthetic probably affects the vagus center which causes cardiac inhibition, respiratory failure and death.

These writers obviate fatal results in anesthetizing hens by injecting subcutaneously in the axilla 1-200 grain of atropine sulphate dissolved in 1 c.c. of warm normal salt solution. Administration of ether follows immediately and the bird will be ready for operation in fifteen to twenty minutes.

\section{ARTHRITIS IN PIGEONS}

Arthritis of the wing joints of pigeons, preventing flight, may be caused by a number of infectious diseases such as tuberculosis or diphtheria; by gout or by mechanical injury. Klee observes that carrier pigeons are subject to the disease under such circumstances as to indicate the existence of a common cause. In affected birds one wing usually droops, for involvement of both wings is rare. On examination the shoulder and elbow joints will be found to be somewhat swollen, reddened and painful. In undertaking treatment, isolation of the bird is absolutely necessary to ensure rest for the joints. He recommends applying a woolen bandage to the wing in such a way as to secure immobility and applying lead water to the same at frequent intervals. The bandage should be changed every 
two days. When heat and swelling disappear after 8 to 14 days, the bandage is removed permanently and the bird is still confined in a cage. The joints may then be painted with tincture of iodine. Cases that do not recover in three weeks may be regarded as hopeless. When caseous masses form in the joints and neighboring tissues they may be removed by operation. Birds thus operated upon seldom fly again and their future usefulness is limited to breeding.

\section{BUMLBLEFOOT}

Bumblefoot is a suppurative condition of the feet of walking birds. It consists of an inflammatory condition involving the bursæ,

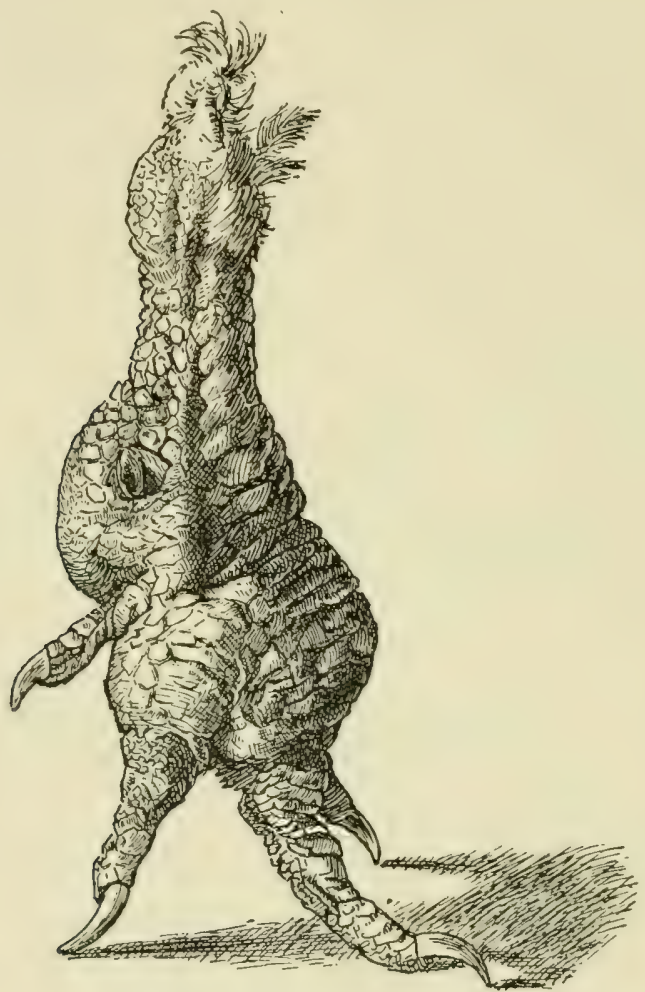

Fig. 55. Bumblefoot. (Klee)

tendon sheaths, and tendons of the foot. It is reputed to occur most often in the heavy breeds and in cocks more often than in hens.

The diseased feet are swollen and hot to the touch. In extreme cases the swelling becomes so large as to interfere with locomotion. 
In some cases fluctuation occurs and in others the tumor remains hard.

Surgical treatment involves incising the bottom of the swelling to allow the escape of the grayish semi-fluid contents. Sometimes the swelling contains a caseous mass of a lamellated structure.

The cavity may be packed with gauze wet with perchloride of iron as a hemostatic and the whole foot bandaged. The bird is confined to an area not provided with a perch. The wound may be dressed every few days, some disinfectant being substituted for the perchloride of iron. Likely the wound will continue to slough, and discharge an ill smelling sceretion. In this event the foot may be bathed in a warm solution of compound solution of cresol. Iodine, zine chloride or lunar caustic may be applied to the interior of the wound. Often treatment may require months, and necessitate trouble out of proportion to the value of the bird.

Bumblefoot has been attributed to injury resulting from jumping from high perehes or to roosting on perehes of an unsuitable, angular character.

\section{CUTANEOUS EMPHYSEMA}

Emphysema of the skin designates a condition in which there is an abnormal amount of air in the subeutaneous region. The

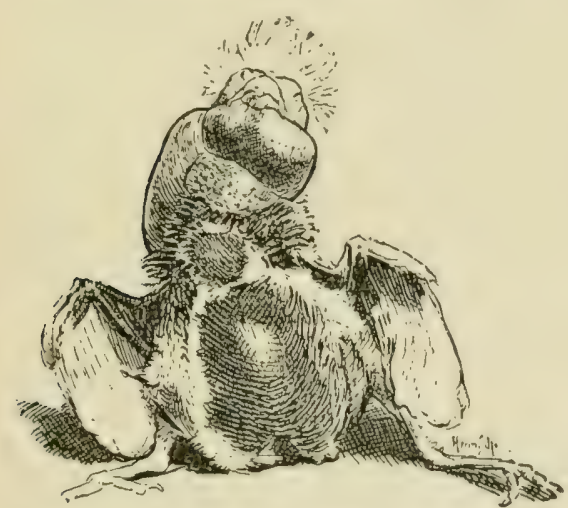

Fig. 56. A young canary bird with emphysema of the skin. (Klee) cause is either a wound or spontaneous rupture of the organs containing air, such as the trachea, or the various air sacs and passages communicating between them. The air imprisoned beneath the skin may so distend the skin that the bird appears almost like a ball.

The condition occurs in young canary birds and has been reported in fowls, turkeys and pigeons.

Radical eorrection of the trouble by preventing the leakage is not prissible, but relief of the condition may be acemplished by slitting the skin to prevent accumulation of air. 


\section{CUTANEOUS HORNY GROWTHS}

Horny growths on the skin of birds are not rare. They result from a proliferation of the epithelial cells and subsequent hardening of the tissue causing deformity. The growths have no effect upon the general health. When the location interferes with function as on the beak, operative treatment is indicated.

In parrots cutaneous horny growths occur, which are of tuberculous origin. Klee reports that skin horns occur in about 50 per cent of parrots affected with tuberculosis.

\section{DUBBING OR CROPPING}

The removal of the comb and wattles is commonly practiced in preparing game birds for the ring. It is also performed upon male breeding birds of some breeds where the comb and wattles are abnormally developed. The removal of these structures is said to improve the condition of the bird and increase the percentage of fertile eggs. The opcration is indicated in bad cases of frost bite and in the infectious disease known as edema of the wattles.

In removing the comb, the bird is held by an assistant who grasps the two legs with one hand and the wings with the other. The operator grasps the comb firmly and cuts with a knife from the base forward to the beak as close to the head as possible. Hemorrhage may usually be controlled by pouring cold water upon the head, but if not, the actual cautery may be applied. Curved surgical scissors may be employed in removing the wattles, which operation does not cause such severe hemorrhage. Antiseptic dusting powders may be applied to the wounds. The bird should be kept secluded until the wounds have entirely healed, before being turned loose among other birds.

\section{FRACTURED BONES}

Broken bones are not rare among fowls as a result of accidents, fighting and mistreatment. Pigeons frequently break wing bones by colliding with objects during flight. Bones of the feet may be fractured when the bird becomes entangled and struggles while hanging by the feet.

A cure is possible and probable if the break is fresh and uncomplicated by splinters, if the soft tissues are not lacerated and if the skin is not punctured. Cure is most probable in simple fractures 
of the bones of the leg and feet. The bird is laid upon the back and the feathers are clipped from the region of the break. The skin is eleaned of blood by washing with three per cent solution of compound solution of cresol. The two broken ends are brought into the eorrect position. $\Lambda$ strip of adhesive tape of the proper dimension is wrapped about the broken bone so that there is formed an enveloping band. Care should be taken not to draw the tape too tight. Pigeons and most sorts of hens do not require a special splint, for the adhesive tape is stiff enough. In the case of very heary boned fowls, ducks and geese it is preferable to use thin strips of wood or an envelope of stiff paper as a foundation for the bandage. Goose quills, softened in hot water and split from end to end, are useful as splints. In the case of weak bones, one quill is enough, but lärger ones need two quills, one on each side. The softened quill fits itself to the bone and is held in place by a bandage. These quill splints are at the same time light and strong and consequently are decidedly preferable to other material.

Fractures of wing bones of pigeons are mostly followed by an impairment of the ability to fly so that treatment is only indicated in valuable individuals which can be utilized as breeders. An adhesive tape bandage is applied after removal of the feathers as in the case of a fracture of a leg bone, only it is useful to make the bandage broader and longer. As soon as the bandage is applied, but while yet soft, it is pressed with the fingers into a flat shape corresponding to that of the wing. The bird is then wrapped with bandages so that both wings lie close to the body and the use of eren the sound one is impossible.

The bandages are kept in place for about two weeks and in that time healing has mostly taken place. To hasten recorery the bird is isolated in a cage without perches or in a crate with planer shavings. In such cases when a bird is confined for a long time, the feathers and cage should be thoroughly dusted with insect powder. The torments suffered by such a bird from blood sucking insects are severe and the restlessness ensuing, retards recovery.

After removal of the bandages, the bird is to be kept isolated for a time from others of his kind for fear that the bone will be broken again.

Swelling of the extremity after bandaging a broken bone indicates that the bandage is too tight and that it must be replaced by a better fitting one. Often in this case it is too late, and it is better to kill 
the patient because in birds dry gangrene oecurs very quickly in parts from which the circulation is cut off. (Klee.)

FROZEN COMB AND WATTLES

When freezing of the appendages of the head occurs, thawing may be accomplished by smearing with vaseline, and manipulating with the fingers. In case of serious injury of the comb or wattles, they may be removed as described under dubbing.

\section{INCISION OF THE CROP}

This operation is frequently necessary in order to evacuate the contents of the organ when impaction is present, and is comparatively harmless in fowls. After removing the feathers from the operative area, an incision is made parallel to the axis of the neck, and as long as the circumstances require. The material is removed by kneading, and the use of forceps. Healing oceurs in about a week after suturing. Feeding must be restricted for a few days to bread and milk but water may be given without limit after 36 hours.

\section{PREVENTION OF FLIGHT}

It is sometimes necessary to deprive birds of the power of flight either temporarily or permanently. To arrive at this result it may be necessary only to cut off the large feathers on one wing, or to prevent the extension of the member by some mechanical device. Whatever the method employed it should be applied to but one wing. The effect in preventing flight is aceomplished more by destroying power of maintaining equilibrium than in reducing wing surface.

Permanent elimination of the primary wing feathers may be accomplished by severing the region of the wing corresponding to the hand. A cut is made with scissors on a line so as barely to leave intact the bone corresponding to the thumb. The proper location for the amputation may be determined by making the cut barely include the primary feathers. Hemorrhage may be arrested by cauterizing or by applying perchloride of iron.

The operation is very simple and quite harmless. Very little trace is left, for the stump is covered by the feathers of the thumb. When the wing is folded, the absence of the primary feathers is not noticeable. 


\section{WOUNDS}

Fowls are liable to be wounded by fighting, by bites of dogs, by birds of prey, etc. Treatment consists of clipping off the feathers in the vicinity to expose the wounded area. The wound may be washed in a three per cent solution of compound solution of cresol. IIemorrhage may be eontrolled by the application of perchloride of iron with a pledget of cotton. Slight hemorrhage may be treated with styptic collodion. Since the blood of birds, especially of pigeons, elots very readily, hemorrhage is usually controlled without difficulty. Extensive wounds of the skin may be sutured. Tattered portions of muscle may be clipped off smooth with scissors.

Treatment is more difficult if the wound includes a tear of the crop. In such a ease the organ must be first emptied of its food contents by flushing out with fluid introduced by means of a rubber tube. Treatment then is carried out in the same manner as in closing an incision of the crop.

In general, wounds of birds, except the most severe ones, heal readily. Suppuration of wounds as in mammals, does not oceur in birds.

\section{REFERENCES}

1. Klee. Die hauptsächlichsten Geflügel-Krankheiten. Leipzig. 1905.

2. Mégnin. Medecine des oiseaux. Vincennes: $\mathrm{Au}$ Bureaux de l'Eleven. 1906.

3. Pearl and Surface. The use of atropine sulphate in anesthetizing birds for surgical experiments. J. Am. M. Ass., Vol. 52, 1909, p. 382. 


\section{CHAPTER XXIV}

\section{CAPONIZING}

\section{CAPONIZING THE FOWL}

The operation of caponizing or castration of the male fowl is described by Slocum as follows:

\section{TIME TO CAPONIZE}

In so far as the effects of the operation and the rapidity and ease of healing are concerned, the time of year when the operation is performed is of little importance. The capons seem to recover and do well at any time. Certain other considerations, however, do influ-

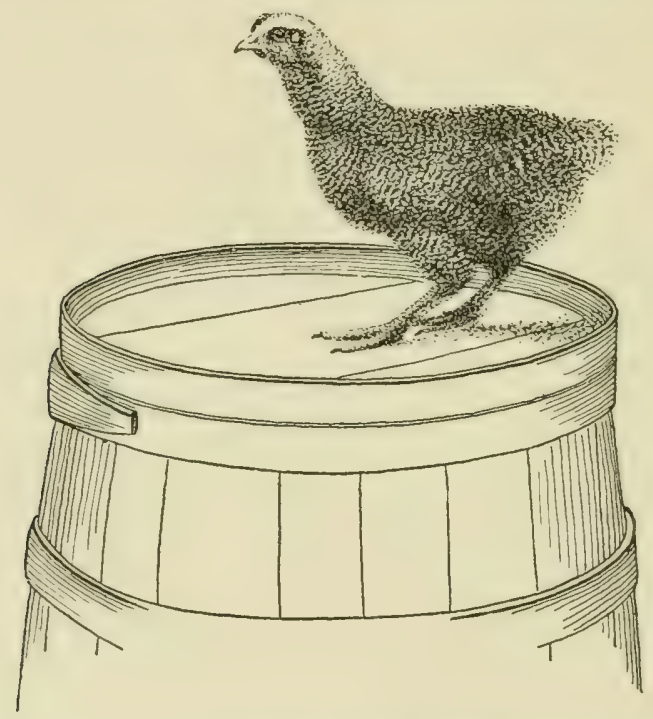

FIG. 57. Barred Plymouth Rock cockerel of suitable size to caponize. (Drawn from photograph by Slocum)

ence the time. The age and size of the cockerel are very important. As soon as the cockerels weigh $1 \frac{1}{2}$ to $2 \frac{1}{2}$ pounds, or when 2 to 4 months old, they should be operated upon. The lower age and weight 
limits apply particularly to the American breeds, while the higher apply to the Asiaties. If smaller than this, their bodies do not give room enough to work handily. On the other hand, they should never be over 6 months old, as by this time the testicles have developed to a considerable extent, the spermatic arteries carry greater amounts of blood, and the danger of pricking these arteries and causing the fowl to bleed to death is greatly increased. The fact that capons are in greatest demand and bring the best prices from the Christmas season until the end of March, and that it takes about 10 months to grow and finish them properly, makes it important to hatch the chicks in early spring so that they will be of proper size for caponizing in June, July, and August. These are by far the most popular months for the operation, though in some cases it is performed still later.

\section{CAPONIZING INSTRUMENTS}

There are several sets of instruments for performing the operation. These differ principally in the type of instrument used in getting hold of and removing the testicle. One type is the cannula (Fig. 58, a). This consists of a hollow tube, the lower end of which is compressed and closed except for two small holes through which to run the horse hair or wire comprising the other part of the instrument. This type requires two hands to operate. Another type is the twisting scoop (Fig. 58, 6). This is a spoon-like scoop slotted in the center and mounted upon a slender rod. It is designed to slip under the testicle, allowing the spermatic cord to pass through the slot. By twisting the eord is severed. This type has the advantage of requiring only one hand to operate, but is more liable to produce "slips" (see p. 309) than the cannula. A third style of instrument (Fig 58, $i$ ) is also in the form of a spoon or scoop, but instead of being in one piece has two jaws regulated by a slide. The testicle is caught in the scoop with the spermatic cord between the jaws, and by tightening the jaws and gently moving the instrument the cord is severed and the testicle removed. Still another type, not now in common use, is the spoon forceps. With this the testicle is simply grasped with the forceps and detached by a twisting movement. Here one hand can be used also, but the liability of slips is rather greater than with the other methods.

Figure 58, $k$, show's a type of forceps, consisting of two hinged arms, one of which terminates in a broad, flat surface, and the other in an end of similar shape from which the center has been remored, 

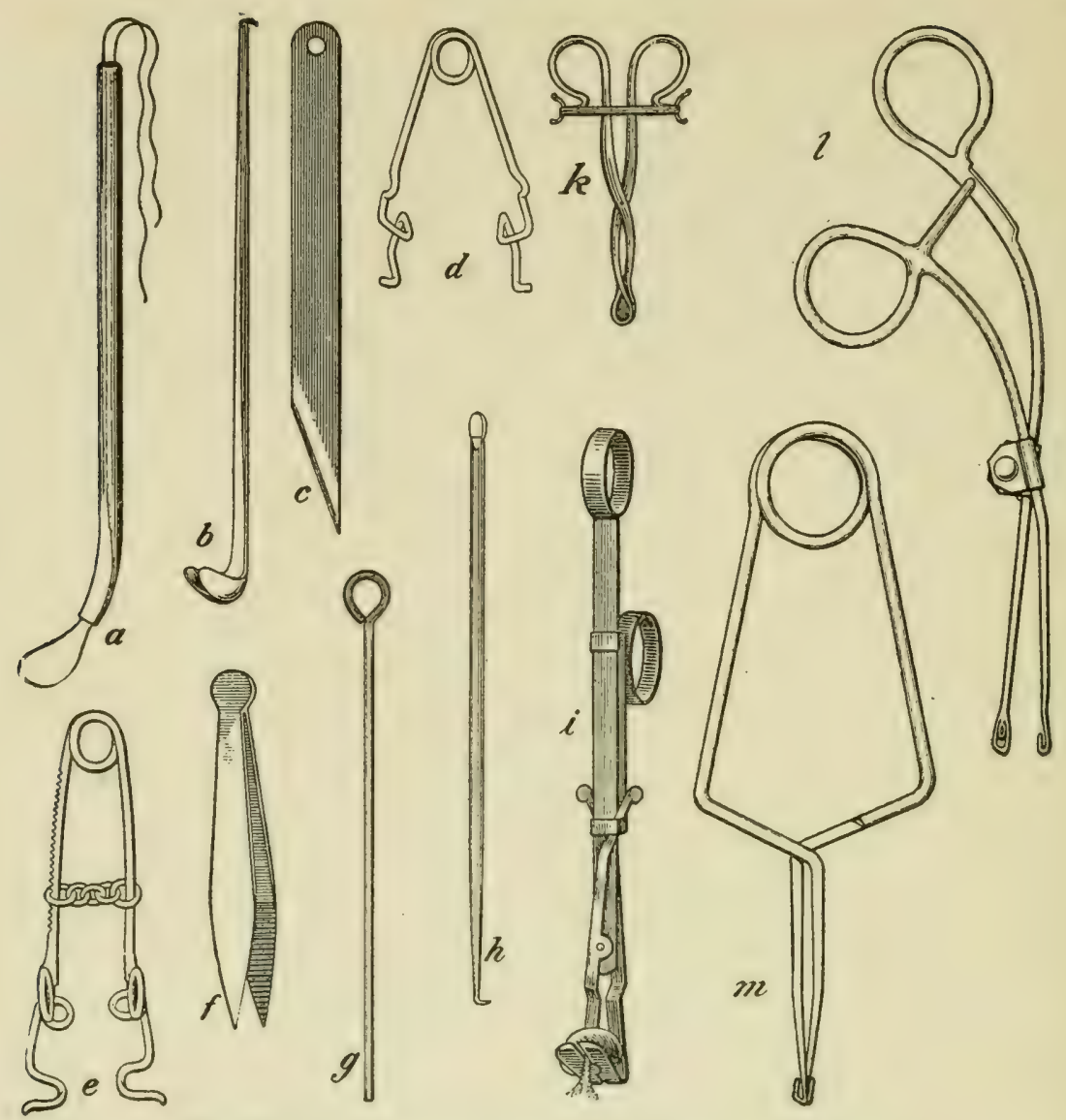

FIG. 58. Instruments used in caponizing. (Slocum)

leaving only a narrow rim. These two ends are held closely pressed together by means of a rubber band passing across the handles. In use, the ends of the forceps are separated, the solid one slipped under the testicle and the rim then allowed to settle down over it. The cord is thus caught and the testicle can be removed. Careless or too rapid use of this instrument is likely to cause slips. Figure 58, $l$ and $m$, shows two additional types of testicle removers. The type shown in $l$ has a curved handle which brings the hand out of the line of vision, making it easier to see into the body cavity when using the instrument. A knife for making the incision into the body cavity is, of course, necessary. Almost any sharp-pointed, thin-bladed knife will answer the purpose well (see Fig. 58, c). Some sort of spreader 
to spring apart the ribs far enough to allow the instruments to be inserted into the body must be used. A plain spring spreader, as shown in tigure $58, d$, or a sliding spreader ( Fig. 58, e), allowing the pressure to be ganged, will answer the purpose. A sharp-pointed hook (Fig. 58,7 ), for tearing away the thin membranes, and a blunt probe, of which figure $58, g$, is one type, for pushing aside the intestines, complete the necessary equipment. A pair of small tweezers or nippers (Fig. $58, f$ ) is also useful in removing any foreign matter from the body.

\section{TIE OPERATION OF CAPONIZING}

Before beginning the operation two conditions are absolutely essential. If these are not favorable, do not attempt to operate. The first of these is that the intestines of the fowl should be completely empty, so that they will fall away and expose the testicle to view. This can be aceomplished by shutting up the fowls and withholding all food and water for 24 to 36 hours before the operation. Withholding water tends to make the blood thicker and consequently to decrease the amount of bleeding. Thirty-six hours is better than 24, especially for a beginner. The second condition is a good, strong light, so that the organs of the fowl may be clearly and easily distinguished. Direct sunlight is best for this, and in consequence it is well to operate out of doors on a bright day. Some operators have substituted the physician's head reflector and artificial light with good success. An ordinary incandescent electric bulb fastened to a gooseneck standard and provided with a reflector can be nsed to gnod advantage when caponizing indoors. It has been suggested that a probe consisting of a small electric bulb on the end of a slender rod and operated by small dry batteries, so that it can be introduced into the body cavity, conld be manufactured and used with good suceess.

\section{METHODS OF HOLDING TIIE FOWL}

When ready to operate, eatch the bird and pass a noose of strong string about the legs. Do the same with both wings elose to the shoulder joints. To the other end of the strings are attached weights of sufficient size to hold down and stretch out the bird when placed upon the head of a barrel or box of convenient height, which is to serve as operating table. These weights are allowed to hang on opposite sides of the barrel or box (Fig. 59). A table, if so desired, may 
be arranged by boring holes through its top at proper distances from each other, allowing the strings to pass through these, and hanging the weights underneath. Still other ways of holding the fowl in place have been devised, but these are unimportant so long as the fowl is held securely stretched out.

\section{DETAILS OF THE OPERATION}

Having fastened the fowl, be sure that all the instruments are at hand. It is also well, though not necessary, to have ready some absorbent cotton and a dish of water to which have been added a few drops of carbolic acid or some other antiseptic. Having once started, carry the operation through as quickly as possible. Moisten and remove the feathers from a small area over the last two ribs just in

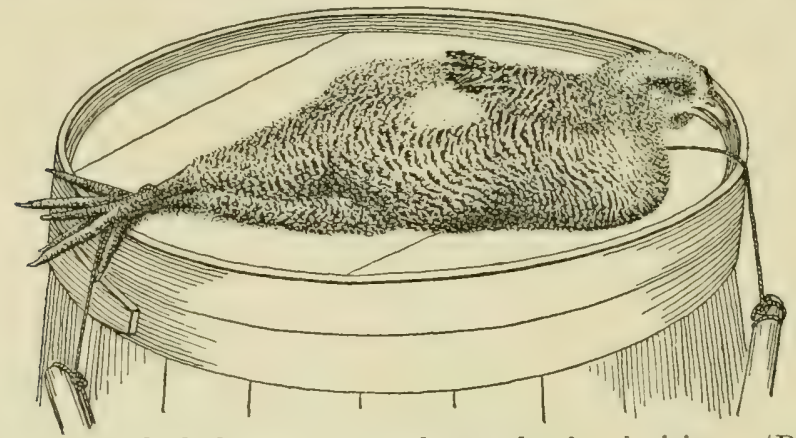

FIG. 59. Feathers plucked away to make ready for incision. (Drawn from photograph by Slocum)

front of the thigh (Fig. 59). With the left hand slide the skin and flesh down toward the thigh. Holding it thus, make the incision between the last two ribs (Fig. 60), holding the edge of the knife away from you as you stand back of the fowl. Lengthen the incision in each direction until it is 1 to $1 \frac{1 / 2}{2}$ inches long. Now insert the spreader into the incision, thus springing the ribs apart, as shown in figure 61 . The intestines will now be visible, covered by a thin membrane called the omentum. Tear apart this membrane with the hook, and the upper testicle, yellow or sometimes rather dark colored and about the size and shape of an ordinary bean, should be visible close up against the backbone. By pushing aside the intestines this can easily be seen, and the lower one also, in a similar position on the other side of the backbone. Expert operators usually remove both testicles through one incision. This is a desirable prac- 


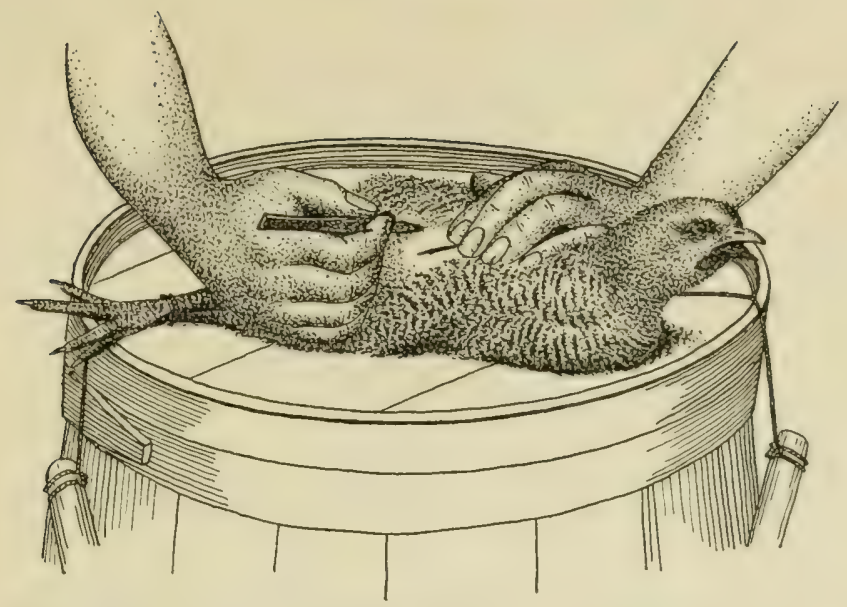

FIG. 60. The incision made. Before making the cut, the skin over the last two ribs is pulled down toward the thigh and held there while the incision is made. When the bird is released after the operation, the skin slips back into its natural position. The eut in the skin is then not directly over the incision in the body, with the result that the wound is closed and protected. (Drawn from photograph by Slocum)

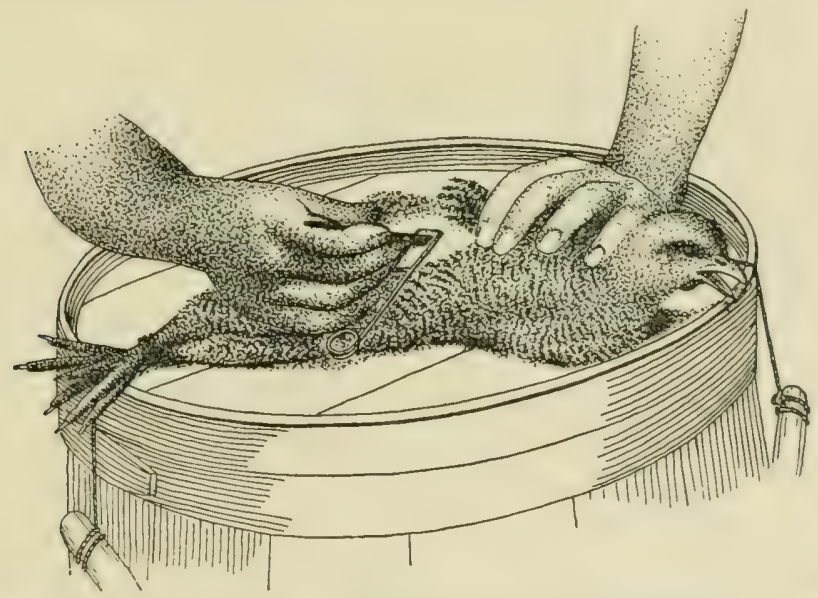

Fia. 61. The spreader in place. Tearing open the membranes. (Drawn from photograph by Slocum)

tice, as it saves time and is not so hard on the bird. Inexperienced operators will usually find it well to attempt the removal of the upper or nearer testicle only and to make a second incision on the opposite side of the body for the removal of the other testicle.

If both testicles are to be removed through the same incision, remove the lower first, as the bleeding from the upuer might be suffi- 


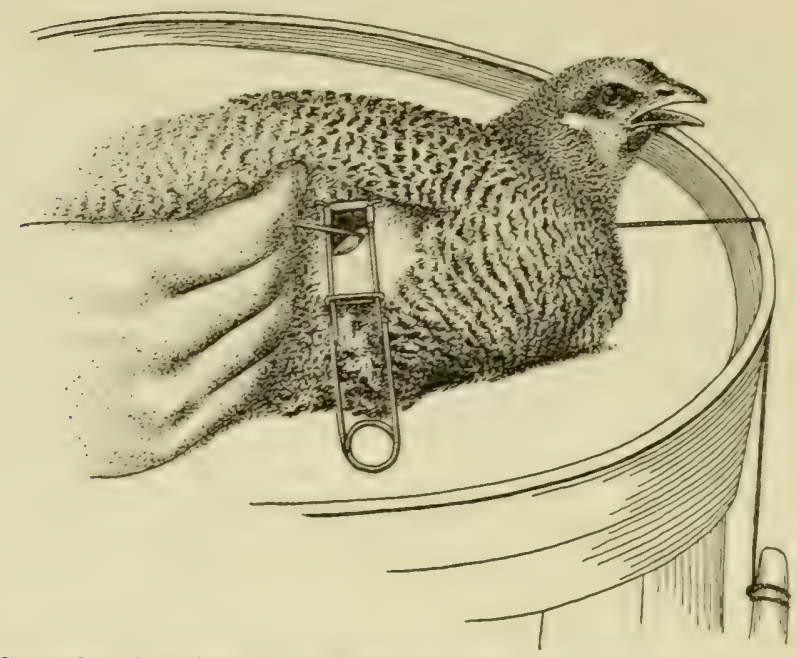

FIG. 62. Spreader in place. The testicle can be observed lying between the jaws of the spreader. (Drawn from photograph by Slocum)

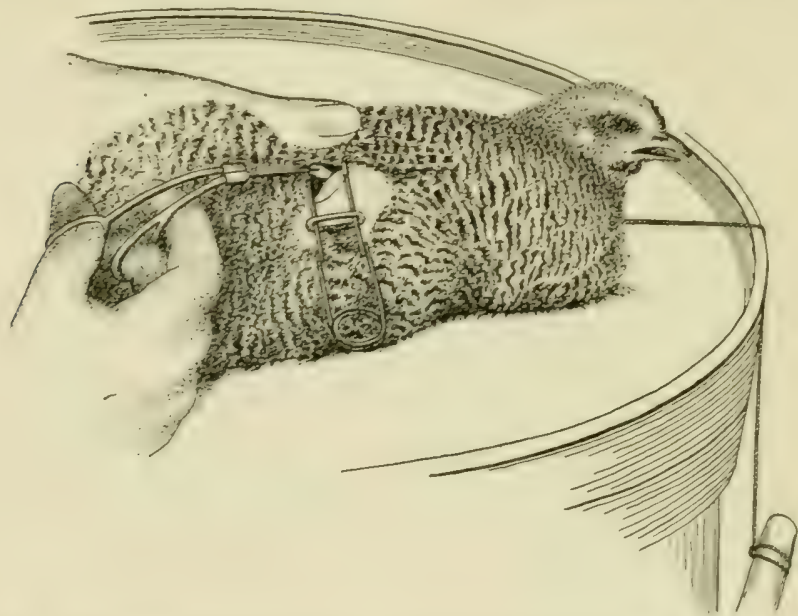

FIG. 63. Removing the testicle. (Drawn from plotograph by Slocum)

cient to obseure the lower. Each testicle is enveloped in a thin membrane. This may be and probably is best removed with the testicle, though some operators tear it open and remove the testicle only.

The delicate part of the operation is now at hand, owing to the close proximity of the spermatic artery, which runs just back of the testicle and to which the testicle is in part attached. If this is ruptured the fowl will bleed to death. The cannula, threaded with a coarse horse- 
hair or fine wire, or one of the other forms of instrument previonily described, now eomes into nse. If the camnula is used, allow the hair or wire protruding from the end to form a small loop just lange enough to slip over the testicle. Work this over the testicle, lueing eareful to inclose the entire organ. Now tighten up on the free ends of the hair or wire being careful not to catch any part of the artery. If the spermatic cord does not separate, saw lightly with

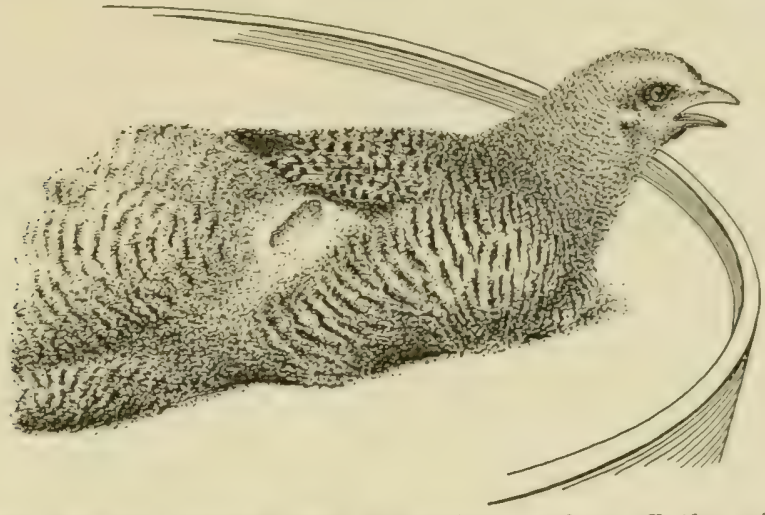

FIG. 64. The spreader removed and the weights taken off the wings. Notice how the skin slips back over the incision so as to close it. (Drawn from photograph by Slocum)

the hair or wire. When the testicle is free, remove it from the body. The method of removing the testicle is shown in figure 63. If only the upper testicle has been removed, turn the bird orer and proceed in exactly the same manner upon the other side.

Ifter remoring the testicle, if the bleeding is at all profuse it is well to remove a portion of the blood by introducing small pieces of absorbent cotton into the body by means of the hook or nippers, allowing them to become saturated and then removing them. Be sure to remove all blood clots, feathers, or foreign matter. After the tegticles and all foreign matter are remored, take out the spreaders, thus allowing the skin to slip back over the incision.

\section{LOSSES DUE TO CAPONIZING}

Eren experts are sure to kill some birds, but the lnss is small, seldom exceeding 5 per cent where any considerable number are caponized, and usually not more than 2 or 3 per cent. With beginners, of course, the percentage is much larger, but with a little 
practice and care this is soon overcome. Any fowls which may be killed in this way are perfectly good to eat and are therefore not wasted.

A great deal of practice is required to become expert enough to operate rapidly. Consequently it is quite common in localities where many capons are grown to hire experts to do the work. These men are able to caponize a fowl every two to five minutes, and charge from 3 to 6 cents a fowl for the service. It is most humane for the beginner to make his first trials upon dead fowls.

\section{SLIPS}

Many times, particularly with beginners, while the operation seems to be entirely satisfactory, the bird will turn out to be what is known as a "slip." A "slip" is neither cockerel nor capon, but is between the two, possessing the mischievous disposition and the appearance of an ordinary cockerel, but, as a rule, being unable to reproduce. This condition is due to the fact that a small piece of the testicle is left in the body. This piece often grows to a considerable size. As the "slips" possess the same restless disposition as the cockerels, they grow and fatten little if any better, while they do not bring as good a price in the market as the capons. Consequently it is well to use every precaution in order to avoid "slips," as they are unprofitable as compared with capons. With the greatest care, however, "slips" are more common than are deaths due to the operation. The percentage varies all the way from 50 per cent with beginners down to 2 or 3 per cent with experts.

\section{CARE OF FOWLS AFTER THE OPERATION}

Upon being released from the operating table the capons are usually put in a closed yard where they can find shelter, food, and water and can be kept quiet. No roosts are provided, as the less flying and jumping they do the sooner will the wound heal. The capons seem to be very little inconvenienced by the operation, and water and soft feed mixed with sweet skim milk can be given immediately. Some feeders give this in unlimited quantity, while others feed more sparingly for a time. Some growers observe no precautions whatever, giving the birds their full liberty immediately after the operation and allowing them to have any sort of feed.

For a week or 10 days the newly made capons should be carefully 
observed to see whether they become "wind puffed." This is a condition caused by air gathering under and puffing out the skin near the wound. When observed it can be readily relieved by pricking the skin with a needle or knife and pressing out the air. In about 10 days or 2 weeks the incision into the body should be entirely healed; and, although no special antiseptic methods are employed in the operation, blood poisoning or any other trouble seldom results.

\section{CAPONIZING THE MALE OSTRICH}

According to Elley, whose description of the operation is given below, the best age for castration of the ostrich is from eighteen months to two years. The testes are then beginning to develop, are easily located, but are not yet so vascular as to occasion any probable risk of hemorrhage.

Preparation for operation. "Have the birds brought into the pen on the afternoon of the day before it is intended to operate, so that they may have no food whatever for say twenty hours before the operation, as the less ingesta in the stomach and bowels, the better and easier it will be. If possible there should be three adjoining pens; the first in which to catch the birds, the second or middle for operating in, and the third in which to place the birds after the operation. The operating pen should be well sprayed with a disinfectant solution before commencing. The first bird is caught and brought to the operating pen and the chloroform bag placed on his head with rather less than half an ounce of chloroform (2-3 teaspoonfuls) on the cotton wool. One good boy can then hold him until he is properly under the influence of the anæsthetic. A little more chloroform can be added after he is down, and if necessary once or twice during the operation. One ounce, or at the most one-and-a-half ounces, should be sufficient for the whole operation. Ascertain by moving the wings or striking him, if he is properly under, then place him on his left side. A boy now grasps the right leg and brings it slightly further forward than at right angles to the body. The seat of the operation is now exposed. It lies in the angle formed by the leg and the lower edge of the innominate bone, about three inches behind the former and one-and-a-half inches below the latter. The innominate bone can be felt as a distinct ridge running backwards and slightly downwards, about the middle of the body immediately behind the leg; it corresponds to the lowest part of the body on which any feathers are found. It is immediately below this ridge that the incision is 
made. The operator should now wash his hands and the seat of the operation with a disinfectant solution.

The operation. Make an incision about four inches long, cutting from before backwards, in a line parallel with the ridge mentioned above. Cut down until the peritoneum or thin membrane covering the bowels is reached. Care is required at this point. Having reached the peritoneum grasp it with the forceps, taking care not to include any of the gut, and draw it slightly towards the external wound, then make an opening through it just large enough to admit the hand. Pour a little carbolic oil over the right hand and force it gently through the opening into the abdominal cavity. The testes are easily located about three to four inches further forward than the incision, almost exactly between the upper extremities of the two legs, situated on and closely attached to the middle of the roof of the abdominal cavity, immediately below the kidneys. In birds of an age which I have recommended for the operation they are felt as two elongated firm structures, about one-and-a-half inches long by a quarter of an inch broad, somewhat the shape of a 303 bullet. They lie about two inches apart, the left usually slightly more forward than the right. Having located them, the tissue round about them is easily broken down by the fingers, and a few twists liberate them and they fall into the palm of the hand. Both testes should be located before either is removed. With care the one can be liberated and held in the palm of the hand whilst the fingers free the other one. It is then only necessary for the hand to enter the abdominal carity once and be once withdrawn, containing both testes. If the birds are in very good condition there will be a certain amount of fatty tissue round the testes which may give a little more trouble in removing them. Having withdrawn them it only remains to suture the wound. Thread a needle with fine cat-gut and put two or three stitches through the peritoneum or membrane taking care not to prick the gut in doing so. Then stitch the external wound with three or four stitches including the skin and muscle. Dress the external wound with a little iodoform and the operation is complete. Whilst the last stitches are being put in, the chloroform bag should have been removed. Allow the bird to lie quietly until he wakes up and let him take his own time about rising, on no account frighten him up or he will invariably attempt to rise before the effects of the chloroform have quite passed off and in falling or struggling the stitches may be broken. Watch the breathing throughout the operation, it should be steady and regular. Should 
it become jerky or irregular, at once open the end of the chloroform bag and admit more air.

After treatment. For the first twenty-four hours after the operation the birds should be placed in as quiet a camp as possible, where they are not likely to be frightened or made to run. Green alfalfa is the best food both before and after the operation.

Instruments. All the instruments required by any one operating by this method are: (a) chloroform bag, such as the one I have had made, (b) sharp knives, (c) needles and cat-gut, (d) needle holder. This latter will be found useful for the deep stitches in the peritoneum.

Advantages resulting from the operation. Having described the operation I will briefly enumerate the advantages which it is claimed will result from it.

1. It is claimed that with the increased condition of the bird which is sure to follow the operation, there will be increased weight of feathers.

2. That owing to the quieter life of the bird the feathers will be less damaged by fighting or scraping on the ground than in the entire bird.

3. In droughts or when food is scarce, castrated birds will keep in fair condition where entire birds would starve.

4. I believe the flesh of a capon will prove an acceptable article of human food. I am quite prepared for this idea to be ridiculed today, but in time to come it may be considered."

\section{REFERENCES}

1. Elley. Castration of ostriches. Agr. J. Cape of Good Hope, Vol. 29,1906, p. 349 .

2. Slocum. Capons and caponizing. U. S. Dep. Agr. Farmers' Bull. 849, 1917. 


\section{CHAPTER XXV}

KILLING POULTRY

Results of bad bleeding. The manner in which fowls are slaughtered and bled out has an important bearing upon the appearance and keeping quality of the product. Poorly bled fowls often show red dots where the feathers have been removed, especially in areas over the thighs and wings. Also, veins are seen to stand out prominently over the breast, angles of the wings, or on the neck. In poorly bled birds the neck is the first part to discolor. At first it is red, later bluish red or purple and still later, green. The bad appearance of poorly bled carcasses detracts from their value and their keeping qualities are very much impaired.

Pennington and Betts have made an anatomical study of the blood vessels of the head and neck of the chicken with reference to determining the most satisfactory method of securing complete bleeding. Their description of proper methods of killing, and discussion of faulty methods are quoted as follows.

\section{" CUTTING" TO BLEED AND "STICKING" TO BRAIN}

When the feathers are removed by scalding, the bird is killed by bleeding alone, hence the cuts to sever the veins are the only ones attempted, and if the attempt fails bad bleeding will surely result. If, on the other hand, dry picking is to be practiced, the birds are cut to bleed and are also stuck throngh the brain to paralyze the feather muscles. The latter operation is sometimes performed by rumning the knife under the eye at such an angle that its point will touch the skull midway between the eyes and a little behind them; or the braining is accomplished by placing the knife about halfway down the groove in the roof of the chicken's mouth, and then thrusting it up until the knife reaches the top of the skull. The knife, as in sticking under the eye, should touch the brain in the back part of the skull. The point of the knife should then be twisted slightly, so that enough brain tissue may be destroyed to paralyze the bird and cause the feathers to loosen. If the " outside-stick" method is practiced, practically no blood escapes. If, on the other hand, braining 
inside of the mouth is adopted, the blood vessels in the brain which are cut find an outlet for their contents through the knife hole. Bleeding from these vessels is, under any circumstances, of assistance in obtaining the best results, and where the neck vessels are missed the condition of the chicken is often greatly improved by the bleeding from the brain.

\section{DISCUSSION OF THE ILLUSTIRATIONS}

\section{LOCATION OF VEINS}

The location of the principal veins in the neck of the chicken, their relation to the skull, and the point at which it is desirable to make the cut for bleeding, are shown in the accompanying illustrations. Figure 65 shows the head and neck of a young cock about

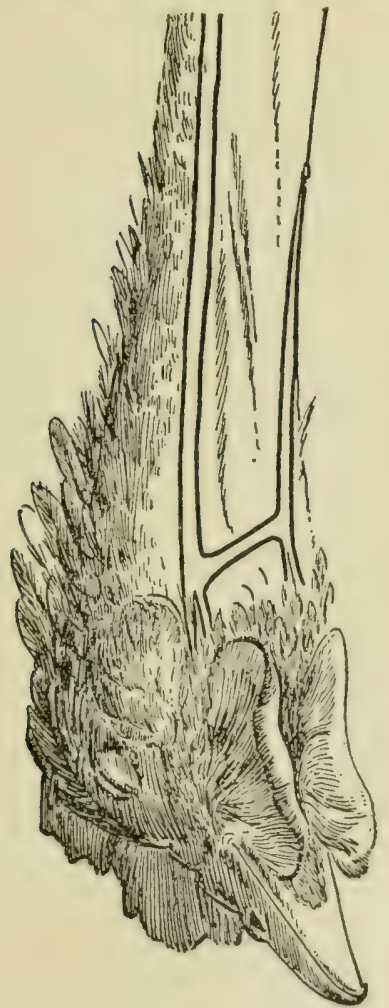

F1G. 65. External view of head and neck showing position of veins. (Pennington and Betts) a year old. Two large veins (heavily lined with black in the figure) run the whole length of the neck, one on each side, and unite by a "bridge-vein" which is just below and behind the ear. This vein, which connects the two others, does not run straight across but at an angle, so that it is farther front on the left than on the right side. If the feathers on the neck of a chicken are pushed aside, or, better still; if a molting chicken with but a few feathers is observed, these veins can be seen at each side of the neck while the bird is alive, especially if a little pressure is used at the lower part of the neck so that the blood collects and distends them. It is important that the position of these veins be exactly located on the neck of the live bird, since upon this information will depend very largely the proper guiding of the knife to the blood vessel.

Fig. 66 shows two sketches of a chicken's head from which the lower jaw has been removed. The lower jaw of the chicken is much longer than the beak. It runs back to a point just below the ear, where the hinged joint ean be felt. The skin which 
makes the corner of the mouth and limits the length of the beak ends is indicated in the anatomical drawing marked "A." The skin and lower jaw have been cut away in order that the position of the veins which lie far back on the roof of the mouth and just below its surface may be seen. The groove which occurs in the roof of the chicken's mouth is a guide to the position of the blood vessel which it is desirable to cut, this point being behind and to the left of the end of the groove when the chicken is held head down and with the lower side of the head uppermost. The direction and position of the cut which
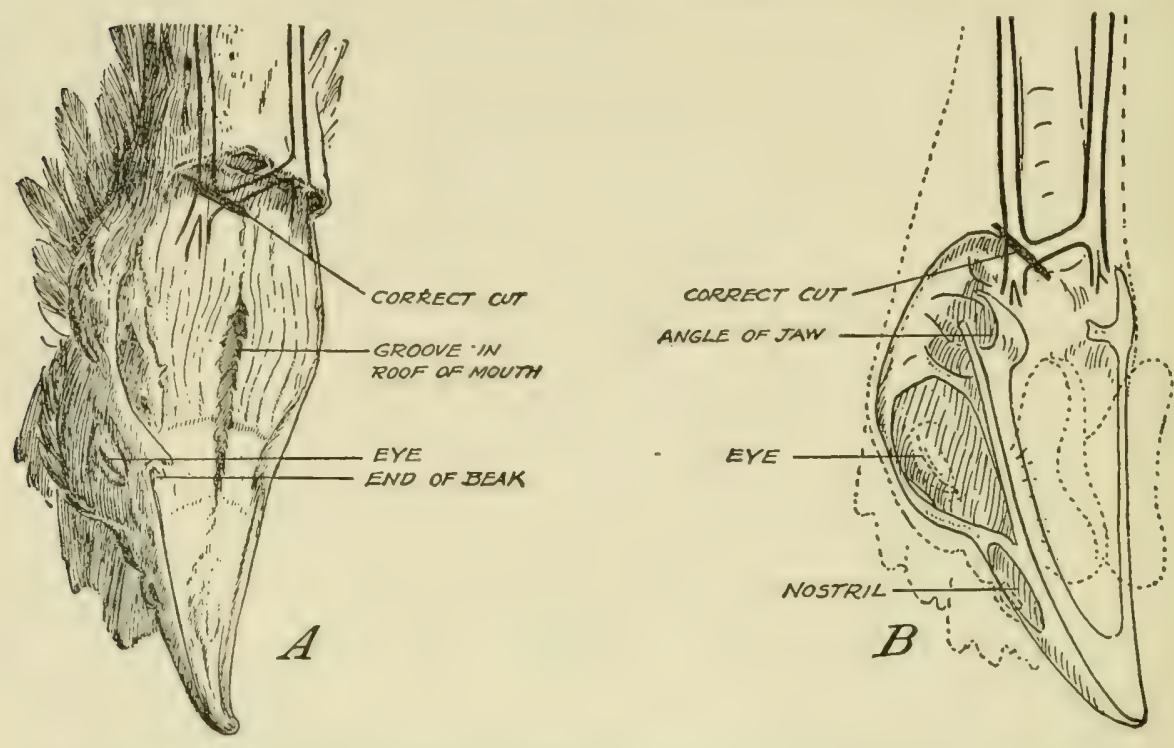

FIG. 66. Lower jaw removed, showing position of veins, anatomy of skull, and location of cut. (Pennington and Betts)

is to sever the veins is shown in Fig. 66 to be on the left side of the chicken's head when in the position just described. Because the short blood vessel connecting the two long veins, which we have termed the "bridge," does not run straight but at an angle, the point just indicated is farthest front and the most easily reached by the knife. As stated before, these veins lie just below the skin of the roof of the mouth, hence a deep cut is not needed, neither is any amount of strength required for the operation.

It will be observed that just in front of the line which indicates the point at which these veins are to be cut they divide into two small branches, the course of which is not further shown. This is because they very soon pass through small holes in the bone and go into the 
inside of the skull, and into the deep tissue, where they are quite safe from the killer's knife. If, then, these large veins are to be severed, the cut must be made far enough back to reach them before they penetrate the bones of the skull. On the other hand, if the cut is made too far back and over the edge of the skull, as will be discussed in connection with Fig. 66, B, much of the blood will settle in the loose tissue of the neck instead of running out of the mouth, thereby clogging the vessels and preventing complete bleeding, as well as making unsightly discolored areas on the neck near the head. It is better to make one cut as shown in this plate rather than to cut the "bridge" in the middle or to cut each side vein separately, since this sometimes results in the clotting of the blood at the ends of the veins before the bleeding is completed.

\section{ANATOMY OF THE SKULL.}

From what has been said concerning the necessity of cutting far back in order to reach the point desired, it will be seen that it is necessary to know something of the position and shape of the bones of the chicken's skull and their relation to the external parts of the head. This information is given in Fig. 66, B. Dotted lines around the drawing of the skull show the position of wattles, comb, etc. In this sketch, too, is shown clearly the length of the chicken's jaw, as before mentioned. Its hinge will be found on the head of the live bird just below the ear. Still farther back there is a U-shaped depression in the skull into which fits the upper part of the spinal column. The spine is not shown in the sketch, but the bridge vein connecting the two large veins of the neck lies directly across the U-shaped depression.

It can be seen by studying this drawing how far back the knife must go if the veins are to be cut at the angle, and it will also be observed that when they are cut in this position the knife will have a bony backing which will prevent it from going too deep, thereby obviating the spongy mass of blood in the neck tissues which was discussed in comnection with Fig. 66, A. This illustration also shows where the fingers can grasp the head of the chicken firmly and yet not press against the soft parts of the neck. Just above the angle of the jaw - that is, about at the chicken's ear - there is a smooth, strong area of bone large enough to support the thumb on one side and the forefinger on the other, and this is where the head should be held while killing. 


\section{POSITION OF HAND AND KNIFE}

Figure 67 shows the position of a chicken ready for killing and held by the feet in a U-shaped shackle. Notice that the thumb of the killer is pressed firmly down on the head just below and behind

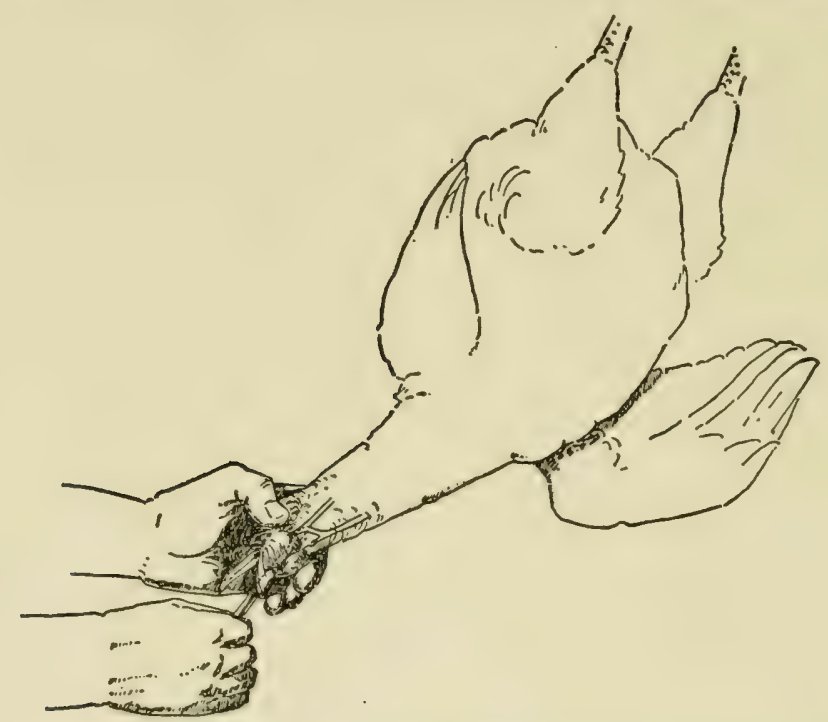

FIG. 67. Correct grasp of head at angle of jaw and position of small knife when cutting vein. (Pennington and Betts)

the ear in the space to which attention was called when discussing the bones of the skull. Here, too, is the hinge of the jaw. Pressure of the thumb on one side of this portion of the skull and on the other side at the same place with the forefinger, or with the forefinger and second finger, will result in opening the chicken's mouth and holding it open while the operator makes the cut to bleed. Held in such fashion, there is nothing to constrict the blood vessels, thereby preventing the blood from escaping even though these vessels be cut. The pressure against the jaw makes accurate cutting of the veins easier, since the bird can not close its mouth until the pressure is removed. Of course, care must be taken not to stretch the neck unduly, else the vessels will be pulled to such a narrow diameter that they are more difficult to find and also more difficult to empty.

The position of the knife in the mouth, which is shown by the dotted line, needs no further explanation. The knife itself, however, is very different from that ordinarily used in the bleeding of 

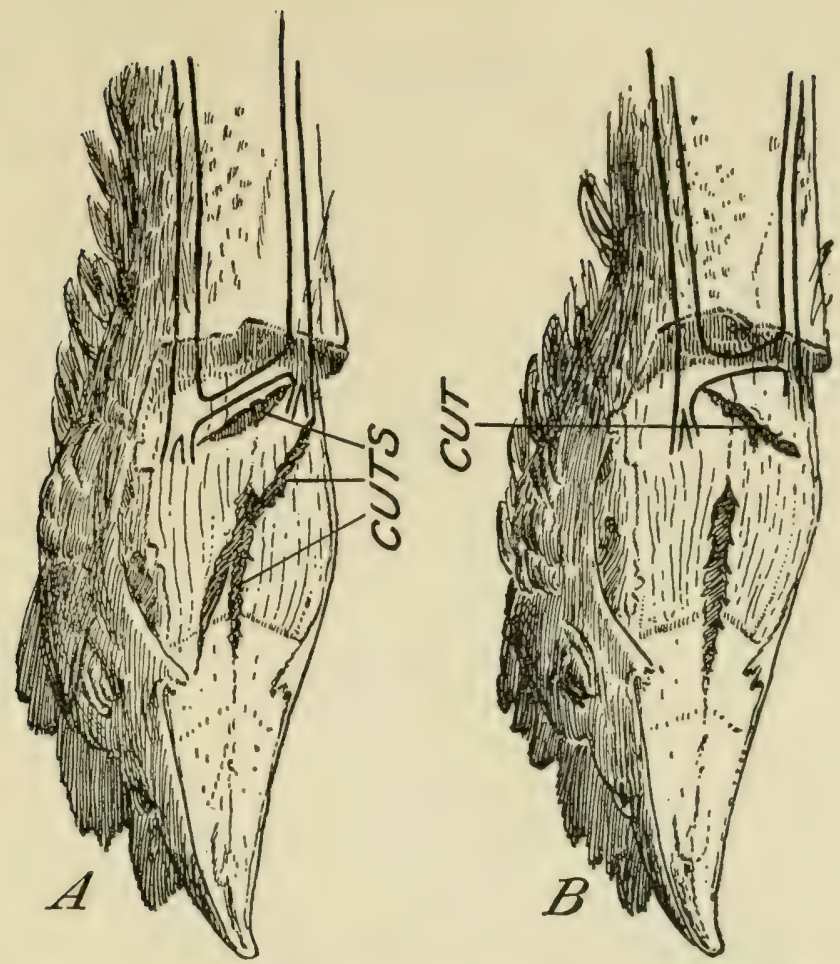

FIG. 68. Location of cuts in mouths of badly bled chickens (lower jaw removed). (Pennington and Betts)

chickens. The knife in common use is much too large, both too long and too broad for the most successful work. Generally it is provided with a heavy handle, large enough to be grasped easily by a large, strong hand. As has already been observed in this discussion, the heavy slashing inside the bird's mouth is not only frequently futile so far as cutting the veins goes, but is really harmful in that it makes a pathway for the entrance of bacteria and the consequent hastening of the bird's decomposition. The operation calls for accuracy rather than for strength, and therefore it is desirable that the knife should have a smaller handle, which can not be gripped so hard. The blade of the knife should be about 2 inches long and onc-fourth of an inch wide and of a heavy piece of stecl, so that it will not bend. It is advisable, therefore, to have the back of the blade about one-sixteenth of an inch thick. It should be made of good hard steel and ground to a sharp point with a straight cutting edge, the slope for the point being taken from the back rather than 
from the edge. The working space in the back part of the mouth of the chicken where the blood vessels lie is very small. Often the knife which is used by the killer is too broad to go into this space without cutting the sides of the mouth, and as for turning and guiding it, that is quite out of the question.

EXAMPLES OF BAD CUTTING

Ineffectual cutting, due to lack of knowledge of the structure of the chicken's neck and head, the use of force rather than skill on the part of the operator, and a knife ill adapted to the work which it has to do, is illustrated in Figs. 68 and 69 which show some of the most common types of cuts in badly bled chickens. A study of these illustrations indicates very plainly why these chickens are badly bled. The lower jaws from these heads were removed so that the position of the cuts could be noted. Head A has had two cuts. One has run

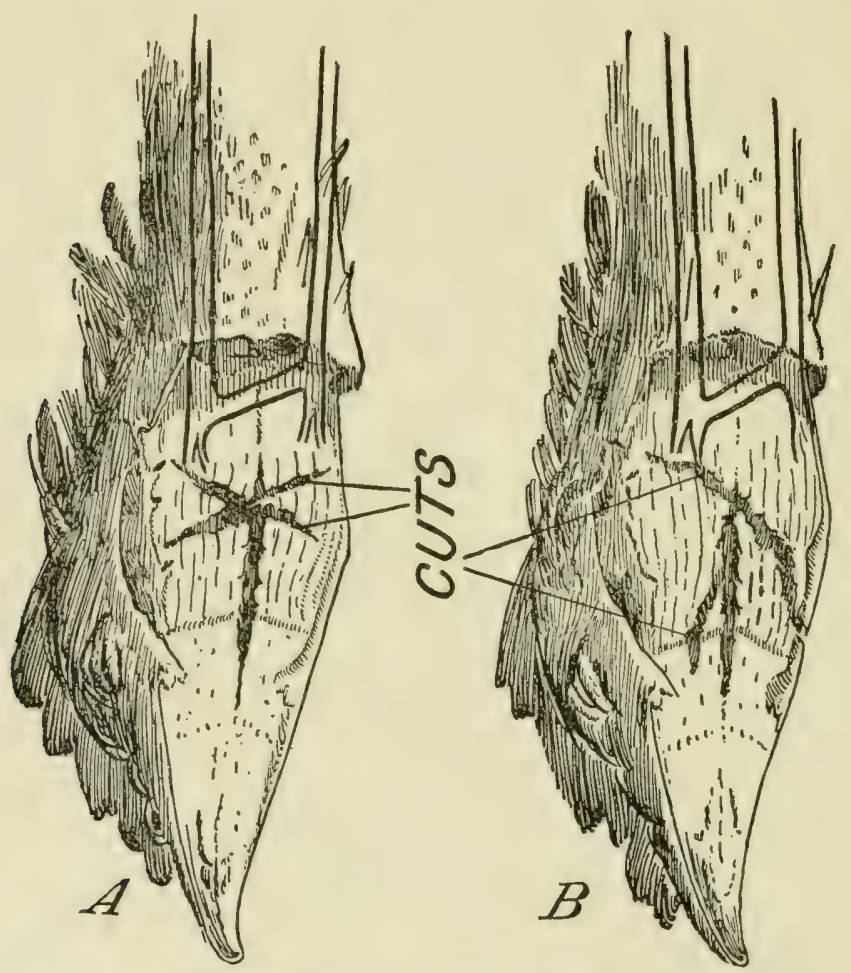

FIG. 69. Attempts at "cross cutting," showing veins untouched. (Pennington and Betts) 
parallel with the connection between the two veins and very elose to it but has not ent it, and another has run from the angle of the mouth to close to the point where the blood vessel on the left side of the head breaks into the two smaller vessels and penetrates the bones of the skull. The only vessels which were cut in this chicken were the small superficial veins supplying the roof of the mouth and from which the blecling amounted to almost nothing. Head B shows a cut in the right direction but it did not go quite far enough back to reach the veins at their junction. Head A, in Fig. 69, shows the cross cut which is advocated by so many killers. In this case it was made too far front. Both of the large veins escaped and only the small vessels of the roof of the mouth were disturbed. " $\mathrm{B}$ " is a good illustration of indiscriminate cutting by a badly directed knife, which in all probability was far too large, since the upper cut extends all the way across the roof of the chicken's mouth and almost as far front as the beak. Another cut which partly follows the groove in the roof of the mouth would indicate that the killer had tried to make a cross cut.

Such examples of bad cutting might be multiplied indefinitely. Yet the general principle is the same and the result is the samenamely, a fowl which is not completely bled, which is unsightly, even in the packing house, and which deteriorates as a food stuff more rapidly than does the well-bled chicken under similar conditions.

\section{SUMMTARY}

The facts which have been stated in the foregoing pages may be summarized as follows:

(1) Grasp the chicken when killing by the bony part of the skull. Do not let the fingers touch the neck.

(2) Make a small cut inside the mouth on the left side of the throat just where the bones of the skull end, using a narrow-bladed sharp-pointed knife. The direction of the knife is upward and toward the left when the bird is held head downward with the throat toward the operator while killing.

(3) Brain for dry picking by thrusting the knife through the groove which runs along the middle line of the roof of the mouth until it pierees the brain in the back part of the skull, causing a loosening of the feathers.

(4) For chickens use a kuife the blade of which is 2 inches long, 
one-fourth inch wide, with a thin, flat handle, a sharp point, and a straight cutting edge. For turkeys the blade may be $2 \frac{1}{2}$ inches long. Keep knives very sharp.

\section{REFERENCE}

1. Pennington and Betts. How to kill and bleed market poultry. U.S. Dep. Agr. Bureau Chemistry Circ. 61, Revised. 


\section{AUTHOR INDEX}

\section{A}

Adam and Medler, 254, 259

Aragão, 141，144，203， 206

Archibald, 229, 240

\section{B}

Balfour, 118

Beach, 109, 189

Beach and Halpin, 118

Beach, Lother and Halpin, 108, 109

Belfanti and Ascoli, 86

Bertegh, 97, 109

Bevan, 136, 144

Binder, 252, 254, 260

Bishopp, 221, 225

Bishopp and Wood, 208, 209, 210, 211, $212,216,218,225$

Bordet and Fally, 107, 109

Borrel, 97

Boyce and Warrington, 19

Bradley, 19

Bugge, 250

Burnet, 19, 109

Burnett, 98

Bushnell and Maurer, 79

Butterfield, 146

\section{C}

Cardamatis, 203, 206

Centanni, 86

Chauveau, 19

Ciurea, 206

Claussen, 61, 65

Cobbold, 63

Cogny, 39

Colueci, 178

Cominotti, 82, 86

Cornil and Toupet, 250

Curtice, 50, 65, 121, 126, 242, 250

Curtis, 261, 274

Cushman, 126

D

Dammann and Manegold, 30, 33
Danielewski, 203

Dawson, 50, 64, 143, 144

Dickson, 154, 177

Dodd, 140

Douglas, 229

Dubois, 86, 143, 144

Dupuy, 65

$\mathrm{E}$

Eastwood and Griffith, 89

Edwards, 290, 294

Ehrenreich, 262, 274

Ehrenreich and Michaelis, 262, 274

Ehrhardt, 142, 144

Ellenberger, 19

Ellenberger and Baum, 19

Ellerman and Bang, 146, 149

Elley, 311, 313

\section{F}

Fantham, 127, 134, 204

Fiorentini, 46, 47, 48

Freese, 83, 86, 190, 246, 247, 250, 255

Friedberger and Fröhner, 97

Frosch and Birnbaum, 243, 250

Fuller, 225

\section{G}

Gage and Hyland, 79

Gage and Martin, 79

Gage and Opperman, 160, 206

Gage and Paige, 80

Gallagher, 16, 39, 48, 109, 163, 276, 294

Gerlach, 199

Gilruth, 254, 260

Görig, 271, 274

Gray, 203, 233, 260

Greve, 31, 33

Gutberlet, 187, 206

H

Hadley, 36, 48, 65, 120, 126, 193, 206

Hadley and Beach, 109 


\section{AUTHOR INDEX}

Haidub, 225

Halasi, 97, 109

Haring and Kofoid, 98, 103, 109

Harrison and Streit, 107, 109

Hasenkamp and Sachweh, 246, 251

Hassall, 225

Hastings and Halpin, 95

Hauer, 140, 144

Hausser, 107, 109

Hebrand and Antoine, 150, 177

Herms and Beach, 189, 206

Herrick, 211, 225

Hertel, 86

Higgins, 48

Hindle, 144

Hutcheon, 238

\section{I}

Iturbe and Gonzales, 204, 206

\section{$J$}

Jackley, 108, 109

Joest, 86, 254, 260

Jones, 75, 77, 80, 274

Jowett, 43, 48, 134, 144, 240

\section{K}

Kaupp, 19

Kern, 260

Kinyoun, 252, 260

Kionka, 151

Kitt, 39, 48

Klee, 177, 189, 197, 295, 298, 300, 301

Klein, 50, 62, 65

Kleine and Moellers, 87

Koch, 263, 265, 274

Koch and Rabinowitsch, 95

Kon, Yutaka, 146

Kraus and Schiffmann, 87

I

Lamson, 287, 294

Lamson and Manter, 211, 225

Landsteiner and Berliner, 87

Lange, 274

Laurie, 225

Leclainche, 45, 48, 87

Leichtenstern, 54, 65

Lewin, 272, 274

Lignières, 61, 66
Lignières and Petit, 119

Lignières and Zabala, 59, 66

Lipschutz, 87

Lisi, 42, 249, 251

Lode and Gruber, 87

Loer, 18, 19

Löftler, 243, 246, 251

Lounsbury, 225

Lucet, $43,48,119,246,247,251$

Mc

M'Fadyeañ, 43, 48, 243, 251

\section{M}

Mack, 109

Mack and Records, 48, 109

Maggiora and Valenti, 87

Magnussen, 28, 33

Mane, 87

Manegold, 31

Manninger, 254, 260

Manteufel, 109

Marchoux and Salimbini, 141, 144

Martel, 61, 66

Martin and Daille, 194, 206

Martin and Robertson, 193, 206

Marx, 231, 232, 233, 240

Marx and Sticker, 97, 98

Matruchot and Dassonville, 119

Mazza, 44, 48

Mégnin, 65, 157, 187, 189, 195, 301

Meyer and Crocker, 134

Miessner and Schern, 252, 260

Milks, 126

Mohler and Buckley, 119

Möllhoff, 143, 144

Moore, 50, 53, 56, 66, 95, 96, 107, 126, 185,206

Morse, 63, 66

Murphy and Rous, 274

\section{$\mathrm{N}$}

Neumann, 119, 183, 188, 192, 194, 200, $202,206,209,220,240$

Nocard and Leclainche, 42, 48

Noguchi, 135, 145

Norgaard and Mohler, 28, 33

$\mathrm{O}$

Ostertag and Bugge, 87 
Ostertag and Wolfhügel, 87

Ottolenghi, 81, 87

Owen, 19

\section{$\mathrm{P}$}

Palamidessi, 66

Pasteur, 39, 48

Pearl and Surface, 295, 301

Pearl, Surface and Curtis, 27

Pennington and Betts, 314, 322

Pereira, 145

Petit and German, 274

Pfaff, 252, 260

Pfeiler, 254, 260

Pfeiler and Rehse, 66

Pfeiler and Roepke, 50, 66

Pick, 262, 274

Pickens, 146, 149, 263, 274

$\mathrm{R}$

Rabieaux, 42, 49

Ransom, 179, 183, 195, 202, 206

Regenbogen, 272, 274

Reinhardt, 278, 294

Reinholdt, 60,66

Reischauer, 98

Repp, 216, 225

Rettger, 80

Rettger and Harvey, 68, 80

Rettger, Hull and Sturges, 80

Rettger, Kirkpatrick and Card, 80

Rettger, Kirkpatrick and Jones, 80

Rettger, Kirkpatrick and Stoneburn, 80

Rettger and Koser, 66

Rettger and Stoneburn, 80

Rieck, 252, 260

Riemer, 243, 251

Ritzler, 280

Robertson, 206, 225, 231, 232, 233, 238, 240, 241

Rous, 268, 269, 274, 275

Rous and Lange, 270, 275

Rous and Murphy, 269, 275

Rous, Murphy and Tytler, 267

Russ, 87

\section{S}

Sabouraud, 119

Sabouraud, Suis and Suffran, 119

Salmon, 27, 220

Sanfelice, 62,66

Schantyr, 161
Scherago and Benson, 79

Schlegel, 275

Schmid, 97

Schneider, 292, 294

Seddon, 41, 49

Sigwart, 97, 107

Slocum, 302, 313

Smith, 120, 126, 193, 242

Smith and Ten Broeck, 66, 69

Steen, 19

Stevenson, 190

Stiles, 206

Suffran, 289, 294

$\mathrm{T}$

Tartakowski, 259, 260

Taylor, 50, 66

Theiler, 226, 241

Theiler and Robertson, 236, 241

Theobald, 189, 198, 206, 207

Tretop, 45, 49

Tytler, 275

Tyzzer and Ordway, 265, 267, 273, 275

U

Uhlenhuth, 140

Uhlenhuth and Manteufel, 97

V

Vages, 39

Van Es and Schalk, 92, 94, 95

W

Walker, 200, 228, 229, 239, 241

Ward, 34, 36, 49, 95, 280

Ward and Gallagher, 80

Warthin, 146, 149, 226, 275

Wasielewski and Hoffmann, 252, 260

Watson, 275

Weil, 40

Weinzirl, 193, 207

Wickware, 205, 207

Wilcox, 200, 207

Willach, 250, 251

Williams, 199, 207

X Y Z

Yutaka Kon, 146

Zeiss, 66, 252, 254, 260 
AUTHOR INDEX

Zeiss and Schlegel, 61

Zürn, 252

Zingle, 57, 66

Zundel, 199

Zürn and Pauly, 272

Zwick, 260 


\section{SUBJECT INDEX}

A

Abdomen, drooping, 173

Acanthia columbara, 214

Acanthia lectularia, 213

Acetabulum, 4

Acorn poisoning in the ostrich, 240

Aggressin for fowl eholera, 40

Air sacs, 15

Air sac mite, 198

Alimentary system, 10 tract, diseases of, 154

Aloes, Barbadoes, 240

Ammonia, 237

Ammonium chloride, 277

Amœba meleagridis, 120, 193

Amœbotænia sphenoides, 183

Analges bifidus, 220

Anesthetizing fowls, 295

Anatomy, 1

Anise oil, 191

Anthrax in the fowl, 143

in the ostrich, 226

vaccine, 227

Apoplectiform septicemia, 28

Areca nut, 187, 190

Argas americanus, 221

miniatus, 221

reflexus, 224

Arsenious acid, toxicity, 278

Arteries, 6

Arthritis in pigeons, 295

Ascaridia perspicillum, 169, 188

Ascaris crassa, 188

Ascites, 173

Aspergillosis in the fowl, 111

in the ostrich, 228

Aspergillus fumigatus, 111, 230

candidus, 112

glaucus, 112

nigressens, 112

Asthenia of fowls, 64

Atropine sulphate, 295

Autopsy directions, 25

Avian diphtheria, 96

salmonellosis, 59

tuberculosis, 88

B

Bacillary white diarrhea, 68
Bacillus avicida, 34

avisepticus, 34

bipolaris septicus, 34

canariensis necrophorus, 253

choleræ gallinarum, 34

coscoroba, 45

enteritidis, 60

gallinarum, 50

loxiacida, 259

paratyphosus B, 57, 58, 60, 252, 254

septicemia anserum exudativæ, 243

suipestifer, 56

Bacterium anthracis, 226

astheniæ, 64

diphtheriæ, 108

pullorum, 52, 68

pullorum infection in grown fowls, 74

sanguinarium, 50

tuberculosis, 88

typhi gallinarum alcalifaciens, 50

Balsam of Peru, 220

Bed bugs, 213

Bertiella delafondi, 183

Bichloride of mercury, disinfectant, 23, 40

toxicity of, 279

Bilharziella polonica, 194

Bird fever, 252

flea, 212

pest, 81

plague, 81

pox, 96

Bismuth subnitrate, toxicity, 280

Blackhead, 120, 193

Blastomyces anseris, 194

Blastomycosis, 194

Blood, 7

platelets, 8

spots in eggs, 169

Blue stone, toxicity, 282

Bones, broken, 298

Boracic acid. 105

Bothriotænia longicollis, 183

Brisket. 3

Bronchitis, 162

Brooder pneumonia, 111

Bumblefoot. 296

Bunodera linearis, 192

Bursa of Fabricus, 13

distention of, 161

parasites of, 194 
C

Calcium oxide, toxicity, 280

Calomel, toxicity, 281

Camphor, powdered, 198

Canary birds, infectious diseases of, 252 fever, 252

Canker, 96

Caponizing cockerels, 302 ostrich, 311

Caraway oil, 219

Carbolic acid, disinfectant, 23

toxicity, 281

vermifuge, 236

Carcinoma, 262

Carpal bones, 4

Caruncle, 18

Castor oil, toxicity, 282

Catarrh, 96

contagious, 96

of crop, 155

Catechu, 133, 282

Ceca, 13

Cercomonas hepatica, 194

Cerebellum, 9

Cestodes, 182

Chalk, powdered, 198

Chicken cholera, 34

Chiggers, 217

Chigoe, 213

Chilomastix gallinarum, 193

Chloride of lime, disinfectant, 23 toxicity, 282

Chlorinated lime, 23

Choanotænia infundibulum, 183, 184

Cholera-like septicemias, 42

Choléra des poules, 34

Cimex columbara, 214

inodorus, 214

lectularia, 213

Circulatory system, 6

Clinostomum commutatum, 192

Cloaca, 13

Cloacitis, 167

Cnemiodocoptes mutans, 218, 219

Coal tar disinfectants, 23

Coccidiosis, 127

Coceidium avium, 127

Colds, 96

Colibacillosis tetraonidarum, 63

Collodion, styptic, 301

Colon bacilli, septicemias caused by, 61

$\mathrm{Comb}, 18$

frozen, 300

Compound solution of cresol, 22

Congestion of lungs, 162

Connective tissue, mites in, 202

Constipation, 160

Contagious catarrh, 96
Copperas, toxicity, 283

Copper sulphate, toxicity, 282

Coracoid, 3

Corrosive sublimate, disinfectant, 23 toxicity, 279

Coryza, 96

Coscoroba swans, 45

Cotugnia diagonopora, 183

Creosote oil, 23

Cresol, compound solution, 22

Crop, anatomy, 10, 12

catarrh of, 155

impaction of, 156

incision of, 300

inflammation of, in pigeons, 156

parasites of, 178

Cropping wattles, 298

Crude carbolic acid, 23 petroleum, 216

Cryptococcus anseris, 194

Cutaneous emphysema, 297 horn, 298

Cyclocœlum arcuatum, 195 mutabile, 192

Cysto-adenoma, 263

Cytodites nudus, 198

Cytoleichus nudus, 198

\section{D}

Daphnia pulex, 181

Davainea cantaniana, 183

cesticillus, 183

crassula, 183

echinobothrida, 183

friedbergeri, 183, 184

proglottina, 183, 184

tetragona, 183

Depluming scabies, 219

Dermaglyphus elongatus, 220

minor, 220

varians, 220

Dermanyssus gallinæ, 214

Dermestes lardarius, 214

Diaphragm, 56

Diarrhea, simple, 159

Digits, 5

Diphtheria, avian, 96

Discolored yolks, 170

Diseases of the alimentary tract, 151 of the oviduct, 164 of the peritoneal cavity, 172 of the respiratory tract, 162

Disinfectants, 22, 23, 24

Disinfecting buildings, 22

Dispharagus hamulosus, 178 laticeps, 178

nasutus, 178

spiralis, 179

uncinatus, 181 
Distention of the bursa of Fabricus, 161

Dithyridium variabile, 200

Docophorus icterodes, 29

Double-yolked eggs, 171

Drooping abdomen, 173

Dropsy, 173

Dubbing wattles, 298

Duck cholera, 250

Ducks, infectious diseases of, 242 septicemia of, 249

Duodenum, 12, 13

\section{E}

Echinococcus polymorphus, 194

Echinorhynchus polymorphus, 193

Echinostomum conoideum, 192 echinatum, 192

filicollis, 192

recurvatum, 192

spærocephalus, 192

Edema of wattles, 41

Egg, blood spots in, 169 bound, 165

double yolked, 171

eating, 176

soft shelled, 171

structure of, 168

Eimeria avium, 127

Epsom salts, 160, 186

Emphysema, cutaneous, 297

Enteritis, 158

Entero-hepatitis of turkeys, 120

Eosinophiles, 8

Epidermoptes bifurcatus, 220

bilobatus, 220

Episternal process, 3

Epithelioma contagiosum, 96

Epizootic dysentery of fowls and turkeys, 43

pneumo-pericarditis in the turkey, 43

Ergot, toxicity, 283

Esophagus, parasites of, 178

External parasites, 208

Exudative septicemia of geese, 243

F

Falculifer anatina, 220

anserina, 220

cornutus, 220

rostratus, 203, 220

Favus, 116

Feathers, 17

Feather crsts, 265

pulling, 176

Femur, 4
Ferrous sulphate, toxicity, 283

Filaria anatis, 203

clava, 203

cygni, 182

mansoni, 201

spicularia, 238

Fimbriaria fasciolaris, 183

Flagellates, 192

Flea, bird, 212

Flight, prevention of, 300

Flukes, 192

Foot and mouth disease, 142

Foot mange, 218

Formaldehyde gas, 22

Formalin, 22

Fowl cholera, 34

fever, 135

plague, 81

tick, 221

typhoid, 50

Fractures of bones, 298

Freyana chanayi, 220

Frozen comb, 300

Furculum, 3, 4

\section{G}

Gangrene of ovary, 75

Gape worms, 195

Gasoline, 211, 238

Gastritis, 157

Geese, infectious diseases of, 242

Geflügelpest, 81

General diseases, 150

Generative system, 16

Gizzard, 12 parasites of, 178

Gonglyonema ingluvicola, 179

Goniocotes abdominalis, 209

chrysocephalus, 209

compar, 209

hologaster, 209

rectangulatus, 209

Goniodes colchicus, 203

damicornis, 209

dissimilis, 209

falcicornis, 209

minor, 209

numidanus, 209

parviceps, 209

stylifer, 209

truncatus, 209

Gout, 150

Grouse disease, 62

H

Habits, vicions, 175

Hæmomœba danielewskyi, 203 
Hæmoproteus columbæ, 203

danielewskyi, 203

Harvest mites, 217

Heart, 6

Hematozoa, 203

Hemiclepsis tessellata, 195

Hemorrhagic septicemia of fowls, 34 of geese, 242 of the ring dove, 45 of the swan, 46

Heterakis brasiliensis, 188 columbæ, 188, 190, 194 compar, 188 compressa, 188

differens, 188

dispar, 188

lineata, 188

papillosa, 188

perspicillum, 188

vesicularis, 188

Holostomum gracile, 192 sphærocephalum, 192

Horn, cutaneous, 298

Hïhnercholera, 34

Hühnerspirillose, 135

Hiihner typhus, 50

Humerus, 4

Hymenolepis anatina, 183 cantaniana, 183

carioca, 183

coronula, 183

fasciata, 183

gracilis, 183

Ianceolata, 183, 184

megalops, 183

meleagris, 183

musculosa, 183

parvula, 183

setigera, 183

sinuosa, 183

tenuirostris, 183

Hygiene, 20

Hystrichis eygni, 181

elegans, 181

tricolor, 181

\section{I}

Ileum, 13

Ilium, 4

Impaction of crop, 156

Incision of crop, 300

Incubating periods, 18

Indigestion, 157 in parrots, 158

Infectious diseases of geese and ducks, 242

enteritis of pheasants, 47
Infectious entero-hepatitis of turkeys; 120

necrosis of canaries, 252

Inflammation of crop in pigeons, 156 of the oviduct, 164

Infraorbital sinuses, 14

Internal parasites, 178

Intestines, 12

Ipecac, toxicity, 283

Ischium, 4

J

Jejunum, 13

K

Keel, 3

Kerosene emulsion, 215

oil, 213

Kidneys, 16

Killing poultry, 314

L

Laminosioptes cysticola, 202

Large mononuclear leucocytes, 8

Larynx, 14

Lead water, 295

Leg weakness, 153

Leiomyoma, 267

Leucocytes, 7

Leucocytozoa, 204

Leucocytozoön anatis, 205

infection in ducks, 205

infection in ostrich, 239

struthionis, 239

Leukemia, 146

Lice, 208

Limberneck, 154

Linseed oil, raw, 240

Liotheum longicaudum, 209

Lipeurus anatis, 209

anseris, 209

baculus, 209

crassicornis, 209

heterographus, 208

meleagridis, 209

numidæ, 209

temporalis, 209

variabilis, 208, 209

Liver, 14 complaint, 88

parasites of, 194

Lophophvton gallinæ, 116

Lungs, 15 conrestion of, 162

Lymphaties, 7

Lymphocytes, 8 
Lymphoma, 265

Lympho sarcoma, 272

Lynchia maura, 204, 213

livicolor, 204

\section{MI}

Macroblasts, 8

Maladie du somneil, 30

Malaria, avian, 203

Male fern, toxicity, 285

Malta fever, 143

Manson's eye worm, 201

Mast cells, 8

Megninia cubitalis, 220

columbæ, 220

ginglymura, 220

velata, 220

Megrims of pigeons, 57

Menopon biseriatum, 208, 209 giganteum, 209

longicephalum, 209

numidxe, 209

obscurum, 209

pallidum, 208

phæostomum, 209

productum, 209

Mercurial ointment, 212

Mercuric chloride, toxicity, 229

Metroliasthes lucida, 183

Metorchis xanthosomus, 194

Microblasts, 8

Micrococcus melitensis, 143

Mierolynchia persilla, 204

Mites, 214

in connective tissue, 202

Monas anatis, 193

Monocercomonas anatis, 193

Touth, 10

Muscular system, 5

Myelogenous leucocytes, 9

Myxo fibroma, 274

sarcoma, 273

\section{$\mathrm{N}$}

Nasal cavity, parasites of, 195

Nervous system, 9

Nest bugs, 214

Nodular tæniasis, 185

Nostrils, 14

Notocotyle verrucosa, 192

Oidium albicans, 155

Opisthorchis simulans, 194

Ornithonomus ergni, 209

Ornithomyia avicularia, 213
Osteo arthritis in geese and ducks, 246

Osteochondrosarcoma, 267

Ostrich, caponizing, 311

diseases of, 226

Ovary, 16, 17

Oviduct, 16

diseases of, 164

Oxyspirura mansoni, 201

\section{$\mathbf{P}$}

Palate, I

Pancreas, 12, 13

Paralysis of the ostrich, 231 of bursa of Fabricus, 194

Parasites, external, 208 internal, 178 of nasal cavity, 195

Parrot septicemia, 54

Parrots, indigestion in, 158

Pasteurella avium, 34

Pasteurellosis avium, 34

Patella, 4

Pectoral muscles, 5

Pelvic bone, 4

Perchloride of iron, 297, 301

Peritoneal cavity, diseases of, 172

Peritonitis, 174

Permanganate of potash, 105

Peste aviaria, 81

Petrol, 238

Petroleum, crude, 216

Phalanges, 5

Pheasants, infectious enteritis in, 47

Phenol, 23

Philopterus cygni, 209

Phosphorus, toxicity, 285

Physaloptera truncata, 178

Pip, 101, 154

Plasmodium danielewskyi, 203

Pneumo-mycosis, 111

Pneumonia, 163 brooder, 111

Poisons, 276

Polymorphonuclear leucocytes, 8

Polyneuritis, 153

Pomegranate root bark, 187

Post-mortem examination, 25

Potassium permanganate, 24, 72 toxicity, 286

Poultry houses, 21

Pox, bird, 96

Prevention of flight, 300

Prolapse of oviduct, 167

Prosthogonimus cuneatus, 195

japonicus. 195

ovatus. 195

in egrs, 169

pellucidus, 179,195 
Proventriculus, 12 parasites of, 178

Pseudo leukemia, 146

Psittacosis of parrots, 54

Pterolichus obtusus, 220

Pterophagus strictus, 220 uncinatus, 220

Pubis, 4

Pulex avium, 212

Pulse rate, 18

Pygostyle, 3

Q

Quail disease, 63

$\mathrm{R}$

Rabies, 142

Rachitis, 152

Red blood corpuscles, 7

Respiration frequency, 19

Respiratory system, 14 tract, diseases of, 162

Rheumatism, 88

Ribs, 3

Ring dove, 45

Rose chafers, toxicity, 287

Round worms, 188

Roup, 96

Rump gland, 17

Rupture of the oviduct, 165

S

Saccharomyces albicans, 155

Salicylic acid, 288

Salmonellosis, avian, 59

Sanitation, 20

Santonin, toxicity, 288

Sarcopsylla gallinacea, 213

Sarcoptes lævis, 219

mutans, 218

Scaly leg, 218

Scapula, 3

Schlafkrankheit, 30

Schlafsucht, 30

Schleg's solution, 220

Sclerostomum anseris, 181

Septic enteritis of cross bills, 259 fever of the canary, 252 of the parrot, 54

Septicemia of ducks, 249 caused by colon bacilli, 61

Shadow cells, 8

Simple diarrhea, 159

Skeleton, 1

Skin, 17

Skull, 1
Sleeping disease, 30

Small mononuclear leucocytes, 8

Sodium chloride, toxicity, 289

fluorid, 210

nitrate, toxicity, 290

salicylate, 197

Soft-shelled eggs, 171

Soil, relation to health, 20

Soor, 155

Spinal cord, 9

Spindle cell sarcoma, 268

Spirochæta anserina, 135 gallinarum, 135

Spirochetosis, 135

Spiroptera emmerzii, 201 pectinifera, 179

Spotted liver, 88

Spur, 5

Staphylococcus pyogenes aureus, 232 , 246

Sternostomum rhinolethrum, 195

Sternum, 3

Stomoxysis calcitrans, 99

Streptococcus, 28 capsulatus gallinarum, 31

Strongylus douglasi, 233 nodularis, 180

quadriradiatus, 190 tenius, 188

Strychnine sulphate, toxicity, 291

Styptic collodion, 301

Suborbital sinuses, 14

Sulphur, flowers of, 211 ointment, 219 toxicity, 293

Swan, hemorrhagic septicemia in, 46 Syngamus bronchialis, 195 trachealis, 195

Syrinx, 15

\section{T}

Tænia exilis, 183

fasciolaris, 183

struthionis. 237

Treniasis, 182

Tapeworm of the ostrich, 237

Tapeworms, 182

Tarso-metatarsal bone, 5

Tartar emetic, toxicity. 293

Temperature, normal, 19

Tenebrio molitor, 214

Thrush, 155

Thorn-headed worms, 192

Thymol, 237

Tilia, 4,5

Ticks, 221

Tohacco, vermifuge, 189

Toe pecking, 175 
Toxicologt, 270

Trachea, 15

Trematodes, 192

Treponema gallinarum, 135

Trichomastix eberthi, 193 gallinarum, 193

Trichomonas, 120 columbx, 192

eberthi, 192

gallinarum, 193

pullorum, 193

Trichomoniasis, 193

Trichosoma anatis, 188

annulatum, 179

caudinflatum, 191

collare, 191

columbæ, 191

contortum, 181

dubium, 191

gallinum, 191

nodularis, 179

retusum, 190

strumosum, 179

Trichostrongylus douglasi, 233 pergracilis, 63

Trinoton anseris, 209

lituratum. 209

luridum, 209

Tropisurus fissipinus, 181

Trypanosomiasis, 203

Tuberculin, avian, 91 test. 91

Tuberculosis, avian, 88

Tumors, 261

Turbinated bones, 14
Turpentine, 186, 294

Ulna, 4

Ureters, 16

Urinary system, 16

Uropygium, 17

V

Veins, 7

Vent gleet, 167

Ventriculus bulbosus, 12

Vertebræ, 1

Vertebral column, 1

Vicious habits, 175

Visceral gout, 150

Vitamines, 153

\section{W}

Wattles, 18

Weisser Kamm, 116

White blood corpuscles, 7

White comb, 116

White diarrhea, 68

Whitewash, 24

Wireworms in the ostrich, 233

Wishbone, 3

Wounds, 301

Y

Yolks, discolored, 170 

THE following pages contain advertisements of a few of the Macmillan books on kindred subjects. 



\section{The Clinical Pathology of the Blood of Domesticated Animals}

By SAMUEL HOWARD BURNETT, A.B., M.S., D.V.M.

Professor in Comparative Pathology, New York State Veterinary College, Cornell University, Ithaca, N. Y.

$$
\text { Ill., Cloth, } 8^{\circ}, \$ 2.25
$$

The purpose of this book is to furnish a text-book of hematology for the use of students and practitioners of veterinary medicine. It is also intended to render easily accessible for investigators data concerning the blood of the kinds of experimental animals commonly used.

\section{TABLE OF CONTENTS}

CHAPTER

I. Methods of Examination.

2. Morphology of the Formed Elements.

3. Normal Blood of the Several Domesticated Animals.

4. Variations in Red Corpuscles and Hemoglobin.

5. Influences AfFecting the LeUcocytes.

6. Special Diseases of the Blood.

7. General and Infectious Diseases.

8. Specific Infectious Diseases Due to Bacteria and Fungt

9. Infectious Diseases Due to Protozoa.

io. Diseases Whose Cause Has Not Been Definitely Deterinined.

i i. Diseases Due to Animal Parasites.

\section{A Text-Book of Parasitology: For Veterinary Students}

By BENJAMIN M. UNDERHILL, V.M.D.

Instructor in Zoölogy, Parasitology and Histology, University of Pennsylvania School of Veterinary Medicine

Preparing.

This work, while designed primarily as a text and reference for students and practitioners in veterinary medicine, will be found a concentrated and handy reference book by practitioners of human medicine, students in zoölogy, and laboratory workers. Heretofore veterinary students in this country have had to rely upon circular matter of this character or upon special chapters scattered in works of other titles.

The work contains little that is historical or critical. Parasitic species uncommon in this country, or pathogenically unimportant, are omitted, though such as are now regarded as rare, but which in the future may become numerous and important, are given warranted consideration.

A feature of the work which marks a distinct advance in the text-book treatment of parasitology is the attention given to the biological side of parasitism. This is essential to the student for his proper conception of the whole subject, and is certainly a prerequisite to intelligently applied measures of parasitic control.

The work fills a long felt want in our veterinary schools, and altogether, it is a valuable and much needed addition to our American veterinary literature.

\section{THE MACMILLAN COMPANY Publishers 64-66 Fifth Avenue New York}




\title{
Practical Veterinary Pharmacology and Therapeutics
}

\author{
By HOWARD JAY MILKS \\ Professor of Therapeutics and Small Animal Clinic, New York State \\ Veterinary College, Cornell University \\ 8vo. $\$ 4.25$
}

This book is designed as a text-book for students and practitioners in veterinary medicine. In addition to the usual discussion on the action and uses of drugs there are chapters on the method of action of drugs, incompatibilities, solubilities, prescription writing and biologic preparations.

Drugs are grouped according to their therapeutic indications or uses and the general therapeutic considerations are taken up just before the members of the group are discussed in detail. Thus the general actions and uses of the group and the detailed study of the drugs belonging to it are discussed in the same chapter. This plan differs from many terts in which the various therapeutic groups are discussed briefly in the first part of the book and to which no reference is made under the detailed discussion of the drugs.

\section{The Diseases of Animals}

\section{BY NELSON S. MAYO}

Cloth, Illustrated, I2mo, $\$ 1.75$

The author gives advice in a brief and popular manner on the diseases and ailments of farm animals. It is a book which will enable those who have the care of animals to detect indications of disease and to make timely application of the necessary remedies.

"It includes about all that the practical keeper of live-stock has need of, and the method of presentation could hardly be better. The exposition has the charm of perfect clearness and simplicity, which makes the book agreeable even to the reader not in search of specific facts." Country Gentleman.

\section{THE MACMILLAN COMPANY}

Publishers 64-66 Fifth Avenue New York 


\title{
The Pathology and Differential Diagnosis of Infectious Diseases of Animals
}

\author{
By VERANUS ALVA MOORE, B.S., M.D., V.M.D.
}

Professor of Comparative Pathology, Bacteriology and Meat Inspection, and Director of the New York State Veterinary College,

Cornell University, Ithaca, N. Y.

Cloth, 8vo, Ill., $\$ 4.00$

This edition has been carefully revised, much of it rewritten and numerous additions made. It has been kept, however, within the limits of a text-book. Two appendices have been added, one on the requirements for interstate shipment of live stock and the other on the Federal regulations for the veterinary inspection of meat. These may be of much assistance to veterinarians. The diseases not indigenous to, or imported into, this country have been accorded much less space than those existing here. The desire is to emphasize the nature of the diseases our veterinarians are liable to encounter and, at the same time, give the characteristics of the others. The same plan of presenting the subject and of grouping the diseases according to their etiology, that was followed in the previous editions, has been retained in this.

\section{Principles of Microbiology: A Treatise on Bacteria Fungi and Protozoa Pathogenic for Domesticated Animals}

\author{
By VERANUS ALVA MOORE, B.S., M.D., V.M.D.
}

Cloth, $8^{\circ}$, Ill., $\$ 4.00$

This volume has been prepared as a text-book for veterinary students beginning the study of microbiology. It is the outgrowth of a lecture course which has accompanied the laboratory work in bacteriology and protozoology. The author has endeavored to choose the material that will be of most help to the student of animal diseases and to avoid as much as possible matters of controversy. In its preparation, the purpose has been to point out the place and rôle of micro-organisms in nature; to give the methods for their study and identification; to indicate the relation of certain species to animal diseases; to give a description of the more important species pathogenic for animals; and a brief discussion of the reaction of the tissues to microbian invasion and the theories of immunity. In order that the volume be of further aid as a key to the entire subject, numerous references to the literature are given where the student can obtain at first hand the results of original research.

\section{THE MACMILLAN COMPANY Publishers 64-66 Fifth Avenue New York}




\title{
Medical and Veterinary Entomology
}

\author{
By WILLIAM B. HERMS
}

Associate Professor of Parasitology in the University of California; Consulting Parasitologist for the California State Board of Health, etc.

Cloth, $8 v 0$, illustrated, $\$ 4.00$

A work of interest to physicians, veterinarians, health officers, and sanitarians as well as to students. Herein is contained a discussion of all the more important insects and arachnids relating to disease and irritations of man and beast. The author has placed special emphasis on control and prevention. He has aimed to familiarize the student with the specific parasite treated in a chapter, its identity, life history, habits, relation to disease transmission or causation, and to indicate methods for its control and prevention. There are 228 illustrations in the text, largely made from original photographs or drawings.

\section{General Surgery}

By DR. EUGEN FRÖHNER

Professor in the Royal Veterinary College in Berlin

Third Revised Edition

Authorized Translation by D. HAMMOND UDALL, B.S.A., V.M.D. Professor of Medicine and Hygiene, N. Y. State Veterinary College, Ithaca; Major, Veterinary Reserve Corps, U. S. Army

Cloth, $8^{\circ}, \$ 3.00$

The new third edition of general surgery is improved and enlarged in many respects. The chapter on etiology of tumors has been rewritten, and many additions made. The chapter on botryomycosis has been newly written to harmonize with the most recent investigations on this subject. The chapter on tuberculosis has been retained and even enlarged. The important chapter on chronic deforming arthritis has been supplemented with the results of recent invertimations made in D. Fröhner's clinic upon ring-bones, chronic gonitis, and omarthritis. Recent investigations on myositis, neuritis, healing of fractures, necrosis bacilli, pseudo-edema bacilli, and foal-lameness have also been considered.

\section{THE MACMILLAN COMPANY Publishers 64-66 Fifth Avenue New York}







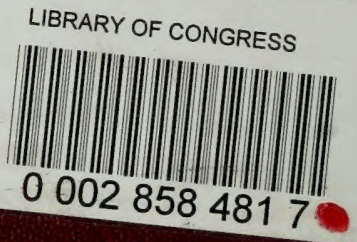

